\title{
Prescribing the behaviour of geodesics in negative curvature
}

\author{
JOUNI PARKKONEN \\ FRÉDÉRIC PAULIN
}

\begin{abstract}
Given a family of (almost) disjoint strictly convex subsets of a complete negatively curved Riemannian manifold $M$, such as balls, horoballs, tubular neighbourhoods of totally geodesic submanifolds, etc, the aim of this paper is to construct geodesic rays or lines in $M$ which have exactly once an exactly prescribed (big enough) penetration in one of them, and otherwise avoid (or do not enter too much into) them. Several applications are given, including a definite improvement of the unclouding problem of our paper [47], the prescription of heights of geodesic lines in a finite volume such $M$, or of spiraling times around a closed geodesic in a closed such $M$. We also prove that the Hall ray phenomenon described by Hall in special arithmetic situations and by Schmidt-Sheingorn for hyperbolic surfaces is in fact only a negative curvature property.
\end{abstract}

53C22, 11J06, 52A 55; 53D25

\section{Introduction}

The problem of constructing obstacle-avoiding geodesic rays or lines in negatively curved Riemannian manifolds has been studied in various different contexts. For example, Dani [16], Stratman [59], Aravinda and Leuzinger [3] and Kleinbock and Weiss [40] have constructed (many) geodesic rays that are bounded (that is, they avoid a neighbourhood of infinity) in noncompact Riemannian manifolds. This work has deep connections with Diophantine approximation problems; see for instance the papers by Sullivan [60], Kleinbock and Margulis [39] and Hersonsky and Paulin [32]. Hill and Velani [34] and others (see for instance Hersonsky and Paulin [30]) have studied the shrinking target problem for the geodesic flow. Schroeder [56] and Buyalo, Schroeder and Walz [13] have worked on the construction of geodesic lines avoiding given subsets; see also the previous work [47] of the authors on the construction of geodesic rays and lines avoiding a uniformly shrunk family of horoballs.

In this paper, we are interested in constructing geodesic rays or lines in negatively curved Riemannian manifolds which, given some family of obstacles, have exactly once an exactly prescribed (big enough) penetration in one of them, and otherwise 
avoid (or do not enter too much into) them. We also study an asymptotic version of this problem. This introduction contains a sample of our results (see also our paper [48]).

Let $H$ be either a horoball of center $\xi$ or a ball of center $x$ and radius $r$ in a CAT(-1) metric space (such as a complete simply connected Riemannian manifold of sectional curvature at most -1 ). For every $t \geq 0$, let $H[t]$ be the concentric horoball or ball contained in $H$, whose boundary is at distance $t$ from the boundary of $H$ (with $H[t]$ empty if $H$ is a ball of radius $r$ and $t>r$ ). The following result (see Section 4.1) greatly improves the main results, Theorem 1.1 and Theorem 4.5, of our note [47]. The fact that the constant $\mu_{0}$ is universal (and not very big, though not optimal) is indeed remarkable.

Theorem 1.1 Let $X$ be a proper geodesic CAT(-1) metric space with arcwise connected boundary $\partial_{\infty} X$ and extendible geodesics, let $\left(H_{\alpha}\right)_{\alpha \in \mathcal{A}}$ be a family of balls or horoballs with pairwise disjoint interiors in $X$, and let $\mu_{0}=1.534$. For every $x$ in $X-\bigcup_{\alpha \in \mathcal{A}} H_{\alpha}$, there exists a geodesic ray starting from $x$ and avoiding $H_{\alpha}\left[\mu_{0}\right]$ for every $\alpha$.

From now on, we denote by $M$ a complete connected Riemannian manifold with sectional curvature at most -1 .

If $M$ has finite volume and $e$ is an end of $M$, let $V_{e}$ be the maximal Margulis neighbourhood of $e$ (see for instance Buser and Karcher [12], Bowditch [10], Hersonsky and Paulin [32] and Section 5.1). If $\rho_{e}$ is a minimizing geodesic ray in $M$ starting from a point in the boundary of $V_{e}$ and converging to $e$, let ht $t_{e}: M \rightarrow \mathbb{R}$ be the height map defined by ht $e_{e}(x)=\lim _{t \rightarrow \infty}\left(t-d\left(\rho_{e}(t), x\right)\right)$. The maximum height spectrum $\operatorname{Max} \operatorname{Sp}(M, e)$ of the pair $(M, e)$ is the subset of $]-\infty,+\infty]$ consisting of elements of the form $\sup _{t \in \mathbb{R}} \mathrm{ht}_{e}(\gamma(t))$ where $\gamma$ is a locally geodesic line in $M$.

As a consequence of Theorem 1.1 (see Corollary 4.4), we prove that if $M$ is noncompact and has finite volume, then there exist universally low closed geodesics in $M$.

From now on, we assume that the dimension of $M$ is at least 3 . The following statements are true or expected to be true in the constant curvature 2-dimensional case, but are expected to be false in variable curvature and dimension 2. We first have the following result on the upper part of the maximum height spectrum.

Theorem 1.2 If $M$ has finite volume and $e$ is an end of $M$, then $\operatorname{Max} \operatorname{Sp}(M, e)$ contains the interval $[4.2,+\infty]$.

For more precise analogous statements when $M$ is geometrically finite, and for finite subsets of cusps of $M$, see Section 5.1. Schmidt and Sheingorn [55] proved the 
two-dimensional analog of Theorem 1.2 in constant curvature -1 . They showed that the maximum height spectrum of a finite area hyperbolic surface with respect to any cusp contains the interval $[4.61,+\infty]$.

The previous result is obtained by studying the penetration properties of geodesic lines in a family of horoballs. Our next theorem concerns families of balls (see Section 5.1 for generalizations). See for instance Hersonsky and Paulin [33] for the almost everywhere properties of the geodesic lines passing at very small distance from a given point.

Theorem 1.3 Let $x$ be point in $M$ with $r=\operatorname{inj}_{M} x \geq 56$. Then, for every $d \in$ $[2, r-54]$, there exists a locally geodesic line $\gamma$ passing at distance exactly $d$ from $x$ at time 0 and remaining at distance greater than $d$ from $x$ at any nonzero time.

Given a closed geodesic $L$ in $M$, the behaviour of a locally geodesic ray $\gamma$ in $M$ with respect to $L$ is typically that $\gamma$ spirals around $L$ for some time, then wanders away from $L$, then spirals again for some time around $L$, then wanders away, etc. Our next aim is to construct such a $\gamma$ which has exactly one (big enough) exactly prescribed spiraling length, and all of whose other spiraling lengths are bounded above by some uniform constant. Let us make this precise.

Let $L$ be an embedded compact totally geodesic submanifold in $M$ with $1 \leq \operatorname{dim} L \leq$ $\operatorname{dim} M-1$, and $\epsilon>0$ small enough so that the (closed) $\epsilon$-neighbourhood $\mathcal{N}_{\epsilon} L$ of $L$ is a tubular neighbourhood. For every locally geodesic line $\gamma$ in $M$, the set of $t \in \mathbb{R}$ such that $\gamma(t)$ belongs to $\mathcal{N}_{\epsilon} L$ is the disjoint union of maximal closed intervals $\left[s_{n}, t_{n}\right]$, with $s_{n} \leq t_{n}<s_{n+1}$. Let $\tilde{\gamma}$ be any lift of $\gamma$ to a Riemannian universal cover of $M$. Let $\widetilde{C}_{n}$ be the lift of $C$ at distance at most $\epsilon$ from $\tilde{\gamma}\left(s_{n}\right)$. Let $p_{\tilde{\gamma}_{-}}$and $p_{\tilde{\gamma}_{+}}$be the orthogonal projections on $\widetilde{C}_{n}$ of the points at infinity of $\tilde{\gamma}$. The distance between $p_{\tilde{\gamma}_{-}}$and $p_{\tilde{\gamma}_{+}}$will be called a fellow-traveling time of $\gamma$ along $L$ (see Section 5.2).

Theorem 1.4 Let $L$ be as above. There exist constants $c, c^{\prime}>0$, depending only on $\epsilon$, such that for every $h \geq c$, there exists a locally geodesic line in $M$, having one fellow-traveling time exactly $h$, all others being at most $c^{\prime}$.

See Section 5.2 for an extension of Theorem 1.4 when $L$ is not necessarily embedded, and to finitely many disjoint such neighbourhoods $\mathcal{N}_{\epsilon} L$. If $M$ has finite volume, we also construct bounded locally geodesic lines with the above property (with a control of the heights uniform in $\epsilon$ ). In constant curvature, we can also prescribe one of the penetration lengths $\left|t_{n}-s_{n}\right|$ at least $c$, while keeping all the other ones at most $c^{\prime}$. Schmidt and Sheingorn [55] sketch a proof of a result for hyperbolic surfaces 
which is analogous to Theorem 1.4 with a different way of measuring the affinity of locally geodesic lines. Other results about the spiraling properties of geodesic lines around closed geodesics are given by Hersonsky and Paulin [33] and Parkkonen and Paulin [49].

For our next result, we specialize to the case where $M$ is a hyperbolic 3-manifold. See Section 5.3 for a more general statement, and for instance Matsuzaki and Taniguchi [44] for references on 3-manifolds and Kleinian groups.

Theorem 1.5 Let $N$ be a compact, connected, orientable, irreducible, acylindrical, atoroidal, boundary incompressible 3-manifold with boundary, with $\partial N$ having exactly one torus component $e$. For every compact subset $K$ in the space $\varphi \mathscr{F}(N, e)$ of (isotopy classes of) complete geometrically finite hyperbolic metrics in the interior of $N$ with one cusp, there exists a constant $c \geq 0$ such that for every $h \geq c$ and every $\sigma \in K$, there exists a locally geodesic line $\gamma$ contained in the convex core of $\sigma$ such that the maximum height of $\gamma$ is exactly $h$.

If $M$ has finite volume and $e$ is an end of $M$, define the asymptotic height spectrum $\operatorname{LimsupSp}(M, e)$ of the pair $(M, e)$ to be the subset of $]-\infty,+\infty]$ consisting of elements of the form $\lim \sup _{t \in \mathbb{R}} \mathrm{ht}_{e}(\gamma(t))$ where $\gamma$ is a locally geodesic line in $M$.

Theorem 1.6 (The ubiquity of Hall rays) If $M$ has finite volume and $e$ is an end of $M$, then $\operatorname{LimsupSp}(M, e)$ contains $[6.8, \infty]$.

The interval given by Theorem 1.6 is called a Hall ray. Note that the value 6.8 is uniform on all couples $(M, e)$, but we do not know the optimal value. If $M$ is the one-ended hyperbolic 2-orbifold $\mathrm{PSL}_{2}(\mathbb{Z}) \backslash \mathbb{H}_{\mathbb{R}}^{2}$ where $\mathbb{H}_{\mathbb{R}}^{2}$ is the real hyperbolic plane with sectional curvature -1 , then the existence of a Hall ray follows from the work of Hall [24; 25] on continued fractions. Freiman [21] (see also Sloane [58]) has determined the maximal Hall ray of $\mathrm{PSL}_{2}(\mathbb{Z}) \backslash \mathbb{H}_{\mathbb{R}}^{2}$, which is approximately $[0.8,+\infty]$. The generality of Theorem 1.6 proves in particular that the Hall ray phenomenon is neither an arithmetic nor a constant curvature property. See Section 5.4 for a more precise version of Theorem 1.6, which is valid also in the geometrically finite case.

The results of Hall and Freiman cited above were originally formulated in terms of Diophantine approximation of real numbers by rationals. The projective action of the modular group $\mathrm{PSL}_{2}(\mathbb{Z})$ on the upper halfplane provides a way to obtain the geometric interpretation. We conclude this sample of our results by giving applications of our methods to Diophantine approximation problems (see Section 6 for generalizations in the framework of Diophantine approximation on negatively curved manifolds, developed by Hersonsky and Paulin $[30 ; 31 ; 32])$. These results were announced in our paper [48]. 
Theorem 1.7 Let $m$ be a squarefree positive integer, and let $\mathcal{I}$ be a nonzero ideal in an order $\mathcal{O}$ in the ring of integers $\mathcal{O}_{-m}$ of the imaginary quadratic number field $\mathbb{Q}(i \sqrt{m})$. For every $x \in \mathbb{C}-\mathbb{Q}(i \sqrt{m})$, let

$$
c(x)=\liminf _{(p, q) \in \mathcal{O} \times \mathcal{I},\langle p, q\rangle=\mathcal{O},|q| \rightarrow \infty}|q|^{2}\left|x-\frac{p}{q}\right|
$$

be the approximation constant of the complex number $x$ by elements of $\mathcal{O I}^{-1}$, and $\mathrm{Sp}_{\text {Lag }}$ the Lagrange spectrum consisting of the real numbers of the form $c(x)$ for some $x \in \mathbb{C}-\mathbb{Q}(i \sqrt{m})$. Then $\mathrm{Sp}_{\text {Lag }}$ contains the interval $[0,0.0005]$.

Theorem 1.7 follows from Hall's result and from the work of Poitou [52] in the particular case $\mathcal{I}=\mathcal{O}=\mathcal{O}_{-m}$. Other arithmetic applications of our geometric methods can be obtained by varying the (nonuniform) arithmetic lattice in the isometry group of a negatively curved symmetric space. We only state the following result in this introduction (with the notation of Section 6.1); see Section 6.4 and our paper [48] for other ones.

Theorem 1.8 Let $\mathcal{Q}(\mathbb{R})$ be the real quadric $\left\{(z, w) \in \mathbb{C}^{2}: 2 \operatorname{Re} z-|w|^{2}=0\right\}$ endowed with the Lie group law $(z, w) \cdot\left(z^{\prime}, w^{\prime}\right)=\left(z+z^{\prime}+w^{\prime} \bar{w}, w+w^{\prime}\right)$ and $\mathcal{Q}(\mathbb{Q})=$ $\mathcal{Q}(\mathbb{R}) \cap \mathbb{Q}(i)^{2}$ be its rational points. If $r=\left(p / q, p^{\prime} / q\right) \in \mathcal{Q}(\mathbb{Q})$ with $p, p^{\prime}, q \in \mathbb{Z}[i]$ relatively prime, let $h(r)=|q|$. Let $d_{\text {Cyg }}^{\prime}$ be the left-invariant distance on $\mathcal{Q}(\mathbb{R})$ such that

$$
d_{\text {Cyg }}^{\prime}((z, w),(0,0))=\sqrt{2|z|+|w|^{2}} .
$$

For every $x \in \mathcal{Q}(\mathbb{R})-\mathcal{Q}(\mathbb{Q})$, let

$$
c(x)=\liminf _{r \in \mathcal{Q}(\mathbb{Q}), h(r) \rightarrow \infty} h(r) d_{\text {Cyg }}^{\prime}(x, r)
$$

be the approximation constant of $x$ by rational points, and $\mathrm{Sp}_{\mathrm{Lag}}$ the Lagrange spectrum consisting of the real numbers of the form $c(x)$ for some $x \in \mathcal{Q}(\mathbb{R})-\mathcal{Q}(\mathbb{Q})$. Then $\mathrm{Sp}_{\mathrm{Lag}}$ contains the interval [0, 0.001].

The paper is organized as follows. In Section 2, we define a class of uniformly strictly convex subsets of metric spaces, that we call $\epsilon$-convex subsets. We study the interaction of geodesic rays and lines with $\epsilon$-convex sets in CAT $(-1)$-spaces. In particular, we give various estimates on the distance between the entering and exiting points in an $\epsilon-$ convex set of two geodesic rays starting from a fixed point in the space and of two geodesic lines starting from a fixed point in the boundary at infinity. Section 3 is devoted to defining and studying several penetration maps which are used to measure the penetration of geodesic rays and lines in an $\epsilon$-convex set. We emphasize the case of penetration maps in horoballs, balls and tubular neighbourhoods of totally geodesic 
submanifolds. We show that in a number of geometrically interesting cases, it is possible to adjust the penetration of a geodesic line or ray in one $\epsilon$-convex set while keeping the penetration in another set fixed. Section 4 contains the inductive construction that gives geodesic rays and lines with prescribed maximal penetration with respect to a given collection of $\epsilon$-convex sets. As a warm-up for the construction, we prove Theorem 1.1 in Section 4.1. The other theorems in the introduction besides the last two and a number of others are proved in Section 5 where the results of Section 4 are applied in the cases studied in Section 3. Finally, we give our arithmetic applications in Section 6.

Acknowledgments Each author acknowledges the support of the other author's institution, where part of this work was done. This research was supported by the Center of Excellence "Geometric analysis and mathematical physics" of the Academy of Finland. We thank P Pansu, Y Bugeaud, A Schmidt, P Gilles, A Guilloux and D Harari for various discussions and comments on this paper.

\section{On strict convexity in $\mathrm{CAT}(-1)$ spaces}

\subsection{Notation and background}

In this section, we introduce some of the objects which are central in this paper. We refer to Bridson and Haefliger [11] and Ghys and de la Harpe [22] for the definitions and basic properties of CAT $(-1)$ spaces. Our reference for hyperbolic geometry is Beardon [5].

Let $(X, d)$ be a proper geodesic CAT $(-1)$ metric space, and $X \cup \partial_{\infty} X$ be its compactification by the asymptotic classes of geodesic rays. By a geodesic line (resp. ray or segment) in $X$, we mean an isometric map $\gamma: \mathbb{R} \rightarrow X$ (resp. $\gamma:\left[\iota_{\gamma},+\infty[\rightarrow X\right.$ with $\iota_{\gamma} \in \mathbb{R}$ or $\gamma:[a, b] \rightarrow X$, with $\left.a \leq b\right)$. We sometimes also denote by $\gamma$ the image of this map. For $x, y$ in $X$, we denote by $[x, y]$ the (unique) closed geodesic segment between $x, y$, with the obvious extension to open and half-open geodesic segments, rays and lines (with one or two endpoints in $\partial_{\infty} X$ ). We say that $X$ has extendible geodesics if every geodesic segment can be extended to a geodesic line.

We denote by $T^{1} X$ the space of geodesic lines in $X$, endowed with the compact-open topology. When $X$ is a Riemannian manifold, the space $T^{1} X$ coincides with the usual definition of the unit tangent bundle, upon identifying a geodesic line $\gamma$ and its (unit) tangent vector $\dot{\gamma}(0)$ at time $t=0$. For every geodesic ray or line $\gamma$, we denote by $\gamma(+\infty)$ the point of $\partial_{\infty} X$ to which $\gamma(t)$ converges as $t \rightarrow+\infty$, and we define $\gamma(-\infty)$ similarly when $\gamma$ is a geodesic line. We say that a geodesic line (resp. ray) $\gamma$ starts from a point $\xi \in \partial_{\infty} X$ (resp. $\xi \in X$ ) if $\xi=\gamma(-\infty)$ (resp. $\gamma\left(\iota_{\gamma}\right)=\xi$ ). For 
every $\xi$ in $X \cup \partial_{\infty} X$, we denote by $T_{\xi}^{1} X$ the space of geodesic lines (if $\xi \in \partial_{\infty} X$ ) or rays (if $\xi \in X$ ) starting from $\xi$, endowed with the compact-open topology.

If $Y$ is a subset of $X$ and $\xi$ a point in $X \cup \partial_{\infty} X$, the shadow of $Y$ seen from $\xi$ is the set $\mathcal{O}_{\xi} Y$ of points $\gamma(+\infty)$ where $\gamma$ is a geodesic ray or line starting from $\xi$ and meeting $Y$.

The Busemann function $\beta_{\xi}: X \times X \rightarrow \mathbb{R}$ at a point $\xi$ in $\partial_{\infty} X$ is defined by

$$
\beta_{\xi}(x, y)=\lim _{t \rightarrow+\infty}(d(x, \rho(t))-d(y, \rho(t))),
$$

where $\rho$ is any geodesic ray ending at $\xi$. The function $y \mapsto \beta_{\xi}(x, y)$ can be thought of as a normalized signed distance to $\xi \in \partial_{\infty} X$, or as the height of the point $y$ with respect to $\xi$ (relative to $x$ ). Accordingly, if $\beta_{\xi}(x, y)=\beta_{\xi}\left(x, y^{\prime}\right)$, then the points $y$ and $y^{\prime}$ are said to be equidistant to $\xi$. If $\xi \in X$, we define

$$
\beta_{\xi}(x, y)=d(x, \xi)-d(y, \xi) .
$$

This is convenient in Section 4.2 and in the proof of Corollary 5.5. For every $x, y, z$ in $X$ and $\xi \in X \cup \partial_{\infty} X$, we have

$$
\beta_{\xi}(x, y)+\beta_{\xi}(y, z)=\beta_{\xi}(x, z),
$$

$\beta_{\xi}(x, x)=0$, and $\left|\beta_{\xi}(x, y)\right| \leq d(x, y)$.

A horoball in $X$ centered at $\xi \in \partial_{\infty} X$ is the preimage of $[s,+\infty$ [ for some $s$ in $\mathbb{R}$ by the map $y \mapsto \beta_{\xi}(x, y)$ for some $x$ in $X$. If

$$
H=\left\{y \in X: \beta_{\xi}(x, y) \geq s\right\}
$$

is a horoball, we define its boundary horosphere by

$$
\partial H=\left\{y \in X: \beta_{\xi}(x, y)=s\right\},
$$

and for every $t \geq 0$, its $t$-shrunk horoball by

$$
H[t]=\left\{y \in X: \beta_{\xi}(x, y) \geq s+t\right\} .
$$

(In [47], we denoted $H[t]$ by $H(t)$.) Similarly, if $B$ is a ball of center $x$ and radius $r$, for every $t \leq r$, we denote by $B[t]$ the ball of center $x$ and radius $r-t$. By convention, if $t>r$, define $B[t]=\varnothing$. Note that for every ball or horoball $H$, we have $H\left[t^{\prime}\right] \subset H[t]$ if $t^{\prime} \geq t$. The point at infinity of a horoball $H$ is denoted by $H[\infty]$. Note that, in this paper, all balls and horoballs in $X$ are assumed to be closed.

Recall that a subset $C$ in a CAT(-1) metric space is convex if $C$ contains the geodesic segment between any two points in $C$. Let $C$ be a convex subset in $X$. We denote by $\partial_{\infty} C$ its set of points at infinity, and by $\partial C$ its boundary in $X$. If $C$ is nonempty and 
closed, for every $\xi$ in $\partial_{\infty} X$, we define the closest point to $\xi$ on the convex set $C$ to be the following point $p$ in $C \cup \partial_{\infty} C$ : if $\xi \notin \partial_{\infty} C$, then $p$ belongs to $C$ and maximizes the map $y \mapsto \beta_{\xi}\left(x_{0}, y\right)$ for some (hence any) given point $x_{0}$ in $X$; if $\xi \in \partial_{\infty} C$, then we define $p=\xi$. This point $p$ exists, is unique, and depends continuously on $\xi$, by the properties of CAT $(-1)$-spaces.

If $x, y, z \in X \cup \partial_{\infty} X$, we denote by $(x, y, z)$ the triangle formed by the three geodesic segments, rays or lines with endpoints in $\{x, y, z\}$. Recall that if $\alpha: t \mapsto \alpha_{t}$ and $\beta: t \mapsto \beta_{t}$ are two (germs of) geodesic segments starting from a point $x_{0}$ in $X$ at time $t=0$, if $\left(\bar{x}_{0}, \bar{\alpha}_{t}, \bar{\beta}_{t}\right)$ for $t>0$ small enough is a comparison triangle for $\left(x_{0}, \alpha_{t}, \beta_{t}\right)$ in the real hyperbolic plane $\mathbb{H}_{\mathbb{R}}^{2}$, then the comparison angle between $\alpha$ and $\beta$ at $x_{0}$ is the limit, which exists, of the angle $\angle_{\bar{x}_{0}}\left(\bar{\alpha}_{t}, \bar{\beta}_{t}\right)$ as $t$ tends to 0 .

If $x, y \in X$ and $\xi \in \partial_{\infty} X$, then a triple $(\bar{x}, \bar{y}, \bar{\xi})$ with $\bar{x}, \bar{y} \in \mathbb{H}_{\mathbb{R}}^{2}, \bar{\xi} \in \partial_{\infty} \mathbb{H}_{\mathbb{R}}^{2}$, $d(\bar{x}, \bar{y})=d(x, y)$ and $\beta_{\bar{\xi}}(\bar{x}, \bar{y})=\beta_{\xi}(x, y)$ is called a comparison triangle for $(x, y, \xi)$. Clearly, this comparison triangle exists, and is unique up to isometry. The natural map from $] \bar{\xi}, \bar{x}] \cup[\bar{x}, \bar{y}] \cup[\bar{y}, \bar{\xi}[$ to $] \xi, x] \cup[x, y] \cup[y, \xi[$ is 1 -Lipschitz, and for every $z \in[x, y]$, if $\bar{z}$ is its corresponding point on $[\bar{x}, \bar{y}]$, then $\beta_{\xi}(z, x) \leq \beta_{\bar{\xi}}(\bar{z}, \bar{x})$.

We end this section with the following (well known) exercises in hyperbolic geometry.

Lemma 2.1 For all points $x, y$ in $X$ and $z$ in $X \cup \partial_{\infty} X$, and every $t$ in $[0, d(x, z)]$ (finite if $z \in \partial_{\infty} X$ ), if $x_{t}$ is the point on $[x, z]$ at distance $t$ from $x$, then

$$
d\left(x_{t},[y, z]\right) \leq e^{-t} \sinh d(x, y) \leq \frac{1}{2} e^{-t+d(x, y)} .
$$

Proof By comparison, we may assume that $X=\mathbb{H}_{\mathbb{R}}^{2}$. As it does not decrease $d\left(x_{t},[y, z]\right)$ to replace $z$ by the point at infinity of the geodesic ray starting from $x$ and passing through $z$, we may assume that $z$ is the point at infinity in the upper halfspace model of $\mathbb{H}_{\mathbb{R}}^{2}$. Let $p$ be the orthogonal projection of $x_{t}$ on the geodesic line $\gamma$ through $y$ and $z$. Assume first that $p$ belongs to $[y, z[$.

If we replace $y$ by the orthogonal projection of $x$ on $\gamma$, then we decrease $d(x, y)$, and do not change $t$ and $d\left(x_{t},[y, z]\right)$. Hence we may assume that $y=i$ and $x$ is on the (Euclidean) circle of center 0 and radius 1 . If $\alpha$ is the (Euclidean) angle at 0 between the horizontal axis and the (Euclidean) line from 0 passing through $x$, then an easy computation in hyperbolic geometry (see also Beardon [5, page 145]) gives $\sinh d(x, y)=\cos \alpha / \sin \alpha$. Similarly, $\sinh d\left(x_{t},[y, z]\right)=\cos \alpha /\left(e^{t} \sin \alpha\right)$. So that

$$
d\left(x_{t},[y, z]\right) \leq \sinh d\left(x_{t},[y, z]\right)=e^{-t} \sinh d(x, y) .
$$




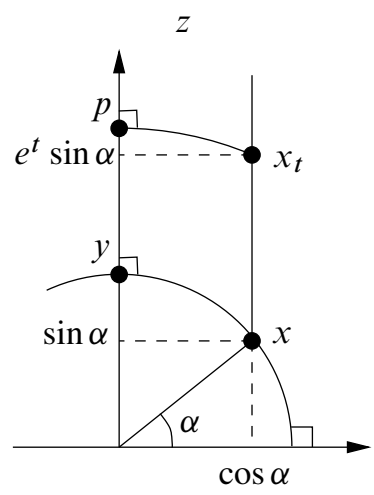

Assume now that $p$ does not belong to $\left[y, z\right.$ [. In particular, $y \neq x_{t}$. Let $\overline{x_{t}}$ be the point at same distance from $y$ as $x_{t}$ (and on the same side) such that $y$ is the orthogonal projection of $\overline{x_{t}}$ on $\gamma$, so that

$$
d\left(\overline{x_{t}},[y, z]\right)=d\left(\overline{x_{t}}, y\right)=d\left(x_{t}, y\right)=d\left(x_{t},[y, z]\right) .
$$

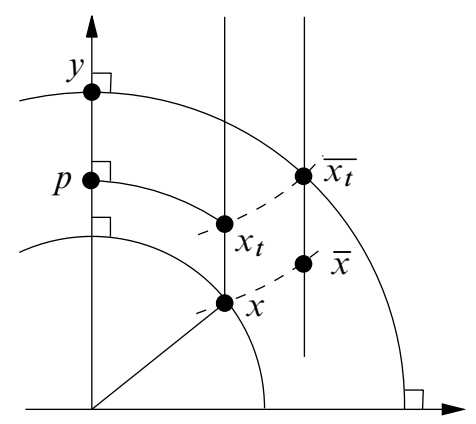

Let $\bar{x}$ be the intersection of the geodesic line from $z$ through $\overline{x_{t}}$ with the (hyperbolic) circle of center $y$ and radius $d(x, y)$, so that $d(\bar{x}, y)=d(x, y)$. Then, with $\bar{t}=$ $d\left(\overline{x_{t}}, \bar{x}\right)$, we have $\bar{t} \geq t$, as the angle at $\overline{x_{t}}$ of $\left[\overline{x_{t}}, \bar{x}\right]$ with the outgoing unit vector of the geodesic ray from $y$ through $\overline{x_{t}}$ is bigger than the corresponding one for $x_{t}$ and $x$. Hence we may assume that $x_{t}=\overline{x_{t}}$ and $x=\bar{x}$. As then the orthogonal projection of $x_{t}$ on the geodesic line through $y$ and $z$ is $y$, this reduces the situation to the first case treated above.

Lemma 2.2 For every $\epsilon>0$, if

$$
c_{0}(\epsilon)=2 \log \left(\frac{2\left(1+e^{\epsilon / 2}\right) \sinh \epsilon}{\epsilon}\right)
$$


then for all points $a, b, a^{\prime}, b^{\prime}$ in $X$ such that

$$
d\left(a, a^{\prime}\right) \leq \epsilon, \quad d\left(b, b^{\prime}\right) \leq \epsilon, \quad d(a, b) \geq c_{0}(\epsilon),
$$

if $m$ is the midpoint of the geodesic segment $[a, b]$, then $d\left(m,\left[a^{\prime}, b^{\prime}\right]\right) \leq \frac{\epsilon}{2}$.

Proof Let $p$ be the point in $\left[a, b^{\prime}\right]$ the closest to $m$, and $q$ the point of $\left[a^{\prime}, b^{\prime}\right]$ the closest to $p$. Let $t=d(a, m)=d(b, m)=d(a, b) / 2$. By Lemma 2.1, we have

$$
d(m, p) \leq e^{-d(b, m)} \sinh d\left(b, b^{\prime}\right) \leq e^{-t} \sinh \epsilon
$$

and, as $d(m, p) \leq \epsilon / 2$ by convexity,

$$
d(p, q) \leq e^{-d(a, p)} \sinh d\left(a, a^{\prime}\right) \leq e^{-d(a, m)+d(m, p)} \sinh d\left(a, a^{\prime}\right) \leq e^{-t+\epsilon / 2} \sinh \epsilon .
$$

Hence $d(m, q) \leq d(m, p)+d(p, q) \leq e^{-t}\left(1+e^{\epsilon / 2}\right) \sinh \epsilon$, and the result follows by the assumption on $d(a, b)$.

Remark If we want a simpler expression, we can also take $c_{0}(\epsilon)=3 \epsilon+4 \log 2$.

\subsection{Entering and exiting $\epsilon-$ convex subsets}

For every subset $A$ in $X$ and $\epsilon>0$, we denote by $\mathcal{N}_{\epsilon} A$ the closed $\epsilon$-neighbourhood of $A$ in $X$. For every $\epsilon>0$, a subset $C$ of $X$ will be called $\epsilon$-convex if there exists a convex subset $C^{\prime}$ in $X$ such that $C=\mathcal{N}_{\epsilon} C^{\prime}$. As the metric space $X$ is CAT(-1), it is easy to see that an $\epsilon$-convex subset $C$ is closed, convex, equal to the closure of its interior, and strictly convex in the sense that for every geodesic line $\gamma$ meeting $C$ in at least two points, the segment $\gamma \cap C$ is the closure of $\gamma \cap \stackrel{\circ}{C}$. If $X$ is a smooth Riemannian manifold, then an $\epsilon$-convex subset has a $C^{1,1}$-smooth boundary; see Walter [62].

Examples (1) For every $\epsilon>0$, any ball of radius at least $\epsilon$ is $\epsilon-$ convex, and any horoball is $\epsilon$-convex. Conversely, as proved below, if a subset $C \subset X$ is $\epsilon$-convex for every $\epsilon>0$, then $C$ is $X, \varnothing$ or a horoball. Accordingly, we will sometimes refer to horoballs as $\infty$-convex subsets.

To prove the above statement, assume that $C \neq X, \varnothing$ and that for all $\epsilon>0$, there exists a convex subset $C_{-\epsilon}$ in $X$ such that $C=\mathcal{N}_{\epsilon} C_{-\epsilon}$. For every $x$ in $\partial C$ (note that $\partial C$ is nonempty as $C \neq X, \varnothing)$ and every $t \geq 0$, let $x_{t}$ be the point of the nonempty closed convex subset $\overline{C_{-t}}$ which is the closest to $x$. Then $t \mapsto x_{t}$ is a geodesic ray, which converges to a point called $x_{\infty}$. We claim that $x_{\infty}=y_{\infty}$ for every $x, y$ in $\partial C$. Otherwise, the geodesic segment between $x_{t}$ and $y_{t}$, contained in $\overline{C_{-t}}$ by convexity, converges to the geodesic line between $x_{\infty}$ and $y_{\infty}$. Hence, the point $x_{t}$ would not 
be the closest one to $x$, for $t$ big enough. Therefore $\partial C$ is a horosphere whose point at infinity is $x_{\infty}$, and by convexity, $C$ is a horoball.

(2) When $X$ is a Riemannian manifold and $C$ is a closed convex subset with nonempty interior and $\mathrm{C}^{1,1}$-smooth boundary, the property of $C$ being $\epsilon$-convex is related with extrinsic curvature properties of its boundary; see for instance our paper [50] and references therein. In particular, if $X$ has constant curvature $-a^{2}$, then $C$ is $\epsilon$-convex if and only if the eigenvalues of the second fundamental form of $\partial C$ (for the inner pointing normal unit vector field along it) belong to $[a \tanh (a \epsilon), a \operatorname{coth}(a \epsilon)]$ almost everywhere; see our paper [50].

The rest of this section is devoted to several lemmas concerning the relative distances between entering points and exiting points, in and out of an $\epsilon$-convex subset of $X$, of two geodesic rays or lines starting from the same point. The asymptotic behaviour of the various constants appearing in this section is described in Remark 2.7.

Lemma 2.3 Let $C$ be a convex subset in $X$, let $\epsilon>0$ and let $\xi_{0} \in\left(X \cup \partial_{\infty} X\right)-$ $\left(\mathcal{N}_{\epsilon} C \cup \partial_{\infty} C\right)$. If two geodesic segments, rays or lines $\gamma, \gamma^{\prime}$ which start from $\xi_{0}$ intersect $\mathcal{N}_{\epsilon} C$, then the first intersection points $x, x^{\prime}$ of $\gamma, \gamma^{\prime}$ respectively with $\mathcal{N}_{\epsilon} C$ are at a distance at most

$$
c_{1}^{\prime}(\epsilon)=2 \operatorname{arsinh}(\operatorname{coth} \epsilon)
$$

Proof Let $y$ and $y^{\prime}$ be the closest points in $C$ to $x$ and $x^{\prime}$ respectively. As $x, x^{\prime} \in$ $\partial \mathcal{N}_{\epsilon}\left[y, y^{\prime}\right]$, it is sufficient to prove the result when $C=\left[y, y^{\prime}\right]$.

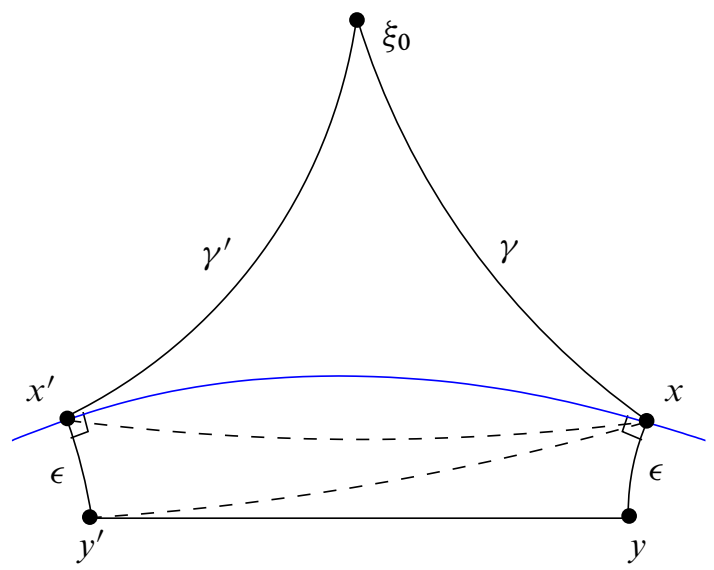

We may assume that $x \neq x^{\prime}$, and, by a continuity argument, that $y \neq y^{\prime}$. Let us construct a pentagon in $\mathbb{H}_{\mathbb{R}}^{2}$ with vertices $\overline{\xi_{0}}, \bar{x}, \bar{y}, \overline{x^{\prime}}, \overline{y^{\prime}}$ by gluing together the comparison 
triangles of $\left(\xi_{0}, x, x^{\prime}\right),\left(x, x^{\prime}, y^{\prime}\right)$ and $\left(x, y^{\prime}, y\right)$. By comparison (see for instance Bridson and Haefliger [11, Proposition 1.7.(4)]), the comparison angles at $\bar{x}, \bar{y}, \overline{x^{\prime}}, \overline{y^{\prime}}$ are at least $\pi / 2$. Hence, the segments or rays $] \overline{\xi_{0}}, \bar{x}[$ and $] \overline{\xi_{0}}, \overline{x^{\prime}}$ [ do not meet $\mathcal{N}_{\epsilon}\left[\bar{y}, \overline{y^{\prime}}\right]$, and the point $\bar{y}$ is the closest point on $\left[\bar{y}, \overline{y^{\prime}}\right]$ to $\bar{x}$.

Furthermore, $\overline{y^{\prime}}$ is the closest point on $\left[\bar{y}, \overline{y^{\prime}}\right]$ to $\overline{x^{\prime}}$. Indeed, the angle at $\overline{y^{\prime}}$ of the pentagon is at most $3 \pi / 2$ since $\angle_{\overline{y^{\prime}}}(\bar{y}, \bar{x}) \leq \pi / 2$ and $\angle_{\overline{y^{\prime}}}\left(\bar{x}, \overline{x^{\prime}}\right) \leq \pi$. Therefore, if by contradiction $\bar{z} \in\left[\bar{y}, \overline{y^{\prime}}\left[\right.\right.$ is closest to $\overline{x^{\prime}}$, the geodesic segment $\left[\bar{x}^{\prime}, \bar{z}\right]$ intersects $\left[\bar{y}^{\prime}, \bar{x}\right]$ at a point $\bar{u}$. If $z \in\left[y^{\prime}, y\right]$ and $u \in\left[y^{\prime}, x\right]$ are such that $d\left(y^{\prime}, z\right)=d\left(\bar{y}^{\prime}, \bar{z}\right)$ and $d\left(y^{\prime}, u\right)=d\left(\bar{y}^{\prime}, \bar{u}\right)$, then by comparison

$d\left(x^{\prime}, z\right) \leq d\left(x^{\prime}, u\right)+d(u, z) \leq d\left(\bar{x}^{\prime}, \bar{u}\right)+d(\bar{u}, \bar{z})=d\left(\bar{x}^{\prime}, \bar{z}\right)<d\left(\bar{x}^{\prime}, \bar{y}^{\prime}\right)=d\left(x^{\prime}, y^{\prime}\right)$,

a contradiction.

As $d\left(x, x^{\prime}\right)=d\left(\bar{x}, \overline{x^{\prime}}\right)$, we only have to prove that $d\left(\bar{x}, \overline{x^{\prime}}\right) \leq c_{1}^{\prime}(\epsilon)$, that is, we may assume that $X=\mathbb{H}_{\mathbb{R}}^{2}$. Up to replacing $\xi_{0}$ by the point at infinity of the geodesic ray starting at $x$ and passing through $\xi_{0}$, we may assume that $\xi_{0}$ is at infinity. By homogeneity, we may assume that $\xi_{0}$ is the point at infinity $\infty$ in the upper halfplane model of $\mathbb{H}_{\mathbb{R}}^{2}$. As a geodesic line starting from $\infty$ and meeting the $\epsilon$-neighbourhood of a vertical geodesic segment enters it in the sphere of radius $\epsilon$ centered at its highest point, we may assume that $C$ is a segment (possibly a point) of the geodesic line $\ell$ between the points -1 and 1 of the real line.

Claim There are points $x_{\sharp}$ and $x_{\sharp}^{\prime}$ that are first meeting points with $\mathcal{N}_{\epsilon} \ell$ of geodesic lines starting from $\infty$, such that $d\left(x, x^{\prime}\right) \leq d\left(x_{\sharp}, x_{\sharp}^{\prime}\right)$.

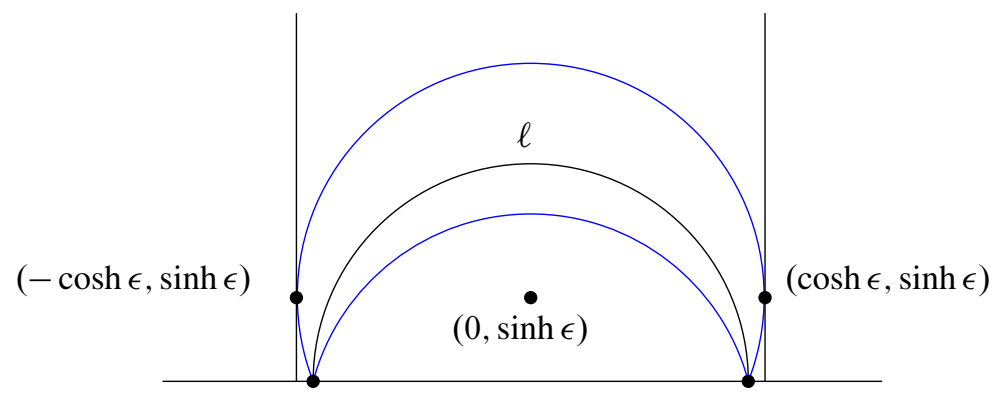

Proof Note that $\partial \mathcal{N}_{\epsilon} \ell$ is the union of two arcs of Euclidean circles, meeting at -1 and 1 , and let $\partial^{+} \mathcal{N}_{\epsilon} \ell$ be the upper one, which is the intersection with $\mathbb{H}_{\mathbb{R}}^{2}$ of the Euclidean circle through $( \pm 1,0)$ and $\left(0, e^{\epsilon}\right)$. The horizontal line with equation $y=\sinh \epsilon$ goes through the Euclidean center $(0, \sinh \epsilon)$ of this circle, and the points 
of $\partial^{+} \mathcal{N}_{\epsilon} \ell$ above or on this line are exactly the first hitting points with $\mathcal{N}_{\epsilon} \ell$ of the geodesic lines starting from $\infty$.

We may assume, up to permuting $x$ and $x^{\prime}$ that the horizontal coordinate of $x$ is strictly less than the one of $x^{\prime}$.

If both $x$ and $x^{\prime}$ are in $\partial \mathcal{N}_{\epsilon} \ell$ or have vertical coordinate at least $\sinh \epsilon$, then replace them by the points $x_{\sharp}$ and $x_{\sharp}^{\prime}$, respectively to their left and right, on $\partial^{+} \mathcal{N}_{\epsilon} \ell$ at the same vertical coordinate. These points satisfy the claim.

Otherwise, assume for instance that $x$ does not lie on $\partial \mathcal{N}_{\epsilon} \ell$ and has vertical coordinate strictly less than $\sinh \epsilon$. In particular, $x$ belongs to the hyperbolic circle $S$ of radius $\epsilon$ centered at one endpoint of the segment $C$. Moreover, the horizontal Euclidean diameter of $S$ is at vertical height strictly less than $\sinh \epsilon$.

Since the Euclidean normal line to $S$ and $\partial^{+} \mathcal{N}_{\epsilon} \ell$ at their common tangency point goes through both their Euclidean centers, this tangency point is below the horizontal Euclidean diameter of $S$. Hence both $x$ and $x^{\prime}$ lie on $S$, since the other points of $\partial \mathcal{N}_{\epsilon} C$ are not first hitting points with $\mathcal{N}_{\epsilon} C$ of the geodesic lines starting from $\infty$. By horizontal translations and homotheties, which are hyperbolic isometries preserving the geodesic lines starting from $\infty$, we may assume that $C$ is reduced to the closest point on $\ell$ to $\infty$. Hence we are again in a situation when both $x$ and $x^{\prime}$ have vertical coordinates at least $\sinh \epsilon$, which has already been considered.

The above claim allows us to assume that $C=\ell$. The distance $d\left(x, x^{\prime}\right)$ is then maximized when the geodesic lines are tangent to $\mathcal{N}_{\epsilon} \ell$ on both sides (see the figure above). Thus, we may assume that the points $x, x^{\prime}$ are $( \pm \cosh \epsilon$, $\sinh \epsilon)$. The computation of $d\left(x, x^{\prime}\right)$ yields the result.

The following technical result will be used in Lemma 2.5. Define, for every $\epsilon>0$,

$$
c^{\prime \prime}(\epsilon)=\frac{2}{\epsilon} \operatorname{arcosh}(2 \cosh (\epsilon / 2)) .
$$

For future use, it is easy to check that, for every $\epsilon>0$,

$$
c_{0}(\epsilon) \geq \epsilon c^{\prime \prime}(\epsilon) \text {. }
$$

Lemma 2.4 For every $\epsilon>0$, for every convex subset $C$ in $X$, for every $a, b$ in $\mathcal{N}_{\epsilon} C$ and for every $a_{0}$ in $[a, b]$, if $d(a, b) \geq c_{0}(\epsilon)$ and

$$
\eta=\frac{1}{c^{\prime \prime}(\epsilon)} \min \left\{d\left(a_{0}, a\right), d\left(a_{0}, b\right)\right\} \leq \frac{\epsilon}{2},
$$

then $d\left(a_{0}, C\right) \leq \epsilon-\eta$. 
Proof Let $\epsilon>0$. Let $C, a, b, a_{0}, \eta$ be as in the statement, and let us prove that $d\left(a_{0}, C\right) \leq \epsilon-\eta$. By an easy computation, we have $c^{\prime \prime}(\epsilon) \epsilon \leq c_{0}(\epsilon)$. By symmetry, we may assume that $d\left(a, a_{0}\right) \leq d\left(b, a_{0}\right)$, so that our assumptions give the following inequalities:

$$
d\left(a, a_{0}\right)=c^{\prime \prime}(\epsilon) \eta \leq c^{\prime \prime}(\epsilon) \epsilon / 2 \leq c_{0}(\epsilon) / 2 \leq d(a, b) / 2 .
$$

Let $a^{\prime}, b^{\prime}$ be the points in $C$ the closest to $a, b$ respectively. As $\left[a^{\prime}, b^{\prime}\right]$ is contained in $C$, we may assume that $C=\left[a^{\prime}, b^{\prime}\right]$. Let $m$ be the midpoint of $[a, b]$, and $m^{\prime}$ its closest point on $\left[a^{\prime}, b^{\prime}\right]$. By Lemma 2.2, we have $d\left(m, m^{\prime}\right) \leq \frac{\epsilon}{2}$.

As $\eta \leq \epsilon / 2$, if $d\left(a, a^{\prime}\right) \leq \epsilon-\eta$, then by convexity every point in $[a, m]$ is at distance at most $\epsilon-\eta$ from $C$. In particular, this is true for $a_{0}$, since $d\left(a, a_{0}\right) \leq d(a, m)$. Hence, we may assume that $d\left(a, a^{\prime}\right)>\epsilon-\eta$.

Consider the quadruple $\left(a, a^{\prime}, m, m^{\prime}\right)$ of points of $X$, which satisfies

- $\epsilon-\eta<d\left(a, a^{\prime}\right) \leq \epsilon$,

- $d\left(m, m^{\prime}\right) \leq \frac{\epsilon}{2}$,

- $a^{\prime}$ is the point in $\left[a^{\prime}, m^{\prime}\right]$ the closest to $a$, and

- $m^{\prime}$ is the point in $\left[a^{\prime}, m^{\prime}\right]$ the closest to $m$.

Define $t=t\left(a, a^{\prime}, m, m^{\prime}\right)$ as the distance between $a$ and the point $z=z\left(a, a^{\prime}, m, m^{\prime}\right)$ in $[a, m]$ at distance $\epsilon-\eta$ from $\left[a^{\prime}, m^{\prime}\right]$ (which exists and is unique by convexity); see the figure below.

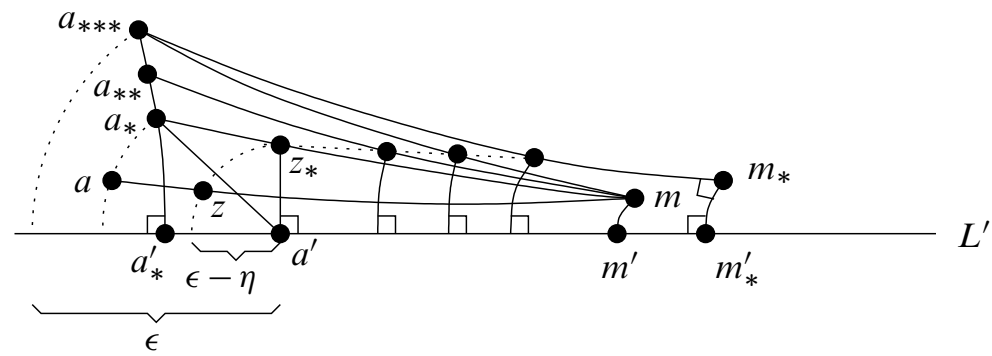

We claim that $t \leq c^{\prime \prime}(\epsilon) \eta=d\left(a, a_{0}\right)$. Before proving this claim, we note that it implies by Equation (3) that $t \leq d\left(a, a_{0}\right) \leq d(a, b) / 2$, hence, by convexity, $d\left(a_{0},\left[a^{\prime}, m^{\prime}\right]\right) \leq$ $\epsilon-\eta$, and Lemma 2.4 will follow.

We will make several reductions, in order to reach a situation where easy computations will be possible. 
First we may assume, by comparison, that $X=\mathbb{H}_{\mathbb{R}}^{2}$. If the segment $[a, m]$ cuts the segment $\left[a^{\prime}, b^{\prime}\right]$ in a point $u$, then replacing $m$ and $m^{\prime}$ by the intersection point $u$ gives a new quadruple with the same $t$. By an approximation argument, we may assume that $[a, m] \cap\left[a^{\prime}, b^{\prime}\right]$ is empty and that $a^{\prime} \neq m^{\prime} \neq m$. The assumptions on the quadruple $\left(a, a^{\prime}, m, m^{\prime}\right)$ then imply that the angles $\angle_{a^{\prime}}\left(a, m^{\prime}\right)$ and $\angle_{m^{\prime}}\left(m, a^{\prime}\right)$ are at least $\frac{\pi}{2}$. Let $L^{\prime}$ be the geodesic line through $a^{\prime}$ and $m^{\prime}$.

If $[a, m]$ does not enter $\mathcal{N}_{\epsilon-\eta} C$ in the sphere $\partial B\left(a^{\prime}, \epsilon-\eta\right)$ (in which case $a$ and $m$ are on the same side of $L^{\prime}$ ), then define $a_{*}=a$. Otherwise, replace $a$ by the point $a_{*}$ at distance equal to $d\left(a, a^{\prime}\right)$ from $a^{\prime}$, such that the geodesic segment between $a_{*}$ and $m$ goes through the point $z_{*} \in \partial B\left(a^{\prime}, \epsilon-\eta\right) \cap \partial \mathcal{N}_{\epsilon-\eta} L^{\prime}$ (on the same side of $L^{\prime}$ as $m$ ). This gives a new quadruple $\left(a_{*}, a^{\prime}, m, m^{\prime}\right)$ satisfying the same properties, whose $t$ has not decreased, by convexity.

Replace $a^{\prime}$ by $a_{*}^{\prime}$ and $a_{*}$ by $a_{* *}$ such that $\angle_{a_{*}^{\prime}}\left(a_{* *}, m^{\prime}\right)=\frac{\pi}{2}, d\left(a_{* *}, a_{*}^{\prime}\right)=d\left(a, a^{\prime}\right)$, and $a_{*} \in\left[a_{* *}, a_{*}^{\prime}\right]$. Clearly, this does not decrease $t$. Now replace $a_{* *}$ by the point $a_{* * *}$ such that $d\left(a_{* * *}, a_{*}^{\prime}\right)=\epsilon$ and $\left[a_{* *}, a_{*}^{\prime}\right] \subset\left[a_{* * *}, a_{*}^{\prime}\right]$. Let $m_{*}$ be the point on $\mathcal{N}_{\epsilon / 2} C$ such that there is a geodesic line through $a_{* * *}$ and $m_{*}$ which is tangent to $\mathcal{N}_{\epsilon / 2} C$ at $m_{*}$. Let $m_{*}^{\prime}$ be its closest point in $L^{\prime}$. Again, the value of $t$ for the quadruple $\left(a_{* * *}, a_{*}^{\prime}, m_{*}, m_{*}^{\prime}\right)$ has not decreased.

Hence, after these reductions, we may assume that $X=\mathbb{H}_{\mathbb{R}}^{2}$, that the quadrilateral $\left(a, a^{\prime}, m, m^{\prime}\right)$ has right angles at $a^{\prime}, m^{\prime}, m$, and that $d\left(a, a^{\prime}\right)=2 d\left(m, m^{\prime}\right)=\epsilon$.

Now, let $\ell=d(m, a)-t$ be the distance between $m$ and the point on $[a, m]$ at distance $\epsilon-\eta$ from $\left[a^{\prime}, m^{\prime}\right]$. An easy computation [5, page 157] shows that

$$
\cosh (t+\ell)=\frac{\sinh (\epsilon)}{\sinh (\epsilon / 2)} \quad \text { and } \quad \cosh \ell=\frac{\sinh (\epsilon-\eta)}{\sinh (\epsilon / 2)} .
$$

Consider the map

$$
f_{\epsilon}: s \mapsto \operatorname{arcosh} \frac{\sinh (\epsilon+s)}{\sinh (\epsilon / 2)} .
$$

This function is increasing and concave on $[-\epsilon / 2,0]$, with $f_{\epsilon}(-\epsilon / 2)=0$. By concavity, the graph of $f_{\epsilon}$ on $[-\epsilon / 2,0]$ is above the line passing through its endpoints $(-\epsilon / 2,0)$ and $\left(0, f_{\epsilon}(0)\right)$. Hence, for every $s$ in $[0, \epsilon / 2]$, by the definition of $c^{\prime \prime}(\epsilon)$, we have $f_{\epsilon}(0)-f_{\epsilon}(-s) \leq c^{\prime \prime}(\epsilon) s$. Therefore $t=f_{\epsilon}(0)-f_{\epsilon}(-\eta) \leq c^{\prime \prime}(\epsilon) \eta$ as $\eta \leq \epsilon / 2$. This proves our claim, and ends the proof of Lemma 2.4.

Here is a finer version of Lemma 2.3 which shows that the entering point of a geodesic which enters an $\epsilon$-convex set for a long enough time and the entering point of any 
nearby geodesic are close. For every $\epsilon>0$, we define

$$
c_{2}^{\prime}(\epsilon)=\max \left\{c^{\prime \prime}(\epsilon)+1, \frac{2 c_{1}^{\prime}(\epsilon)}{\epsilon}, \sqrt{\frac{\cosh \epsilon}{\cosh \epsilon-1}} \frac{\sinh c_{1}^{\prime}(\epsilon)}{c_{1}^{\prime}(\epsilon)}\right\} .
$$

Lemma 2.5 For every $\epsilon>0$, every $\xi_{0}$ in $X \cup \partial_{\infty} X$, every convex subset $C$ in $X$, and all geodesic rays or lines $\gamma, \gamma^{\prime}$ in $X$ which start at $\xi_{0}$ and enter $\mathcal{N}_{\epsilon} C$ at the points $x, x^{\prime}$ in $X$ respectively, if the length of $\gamma^{\prime} \cap \mathcal{N}_{\epsilon} C$ is at least $c_{0}(\epsilon)$, then we have

$$
d\left(x, x^{\prime}\right) \leq c_{2}^{\prime}(\epsilon) d\left(x, \gamma^{\prime}\right) .
$$

Remarks (1) Without assuming that the geodesic ray or line $\gamma^{\prime}$ has a sufficiently big penetration distance inside $\mathcal{N}_{\epsilon} C$, the result is false, as can be seen by taking $C$ a point, $\gamma^{\prime}$ a geodesic line tangent to $\partial \mathcal{N}_{\epsilon} C$ and $x$ very close to $x^{\prime}$ on $\partial \mathcal{N}_{\epsilon} C$.

(2) The curvature assumption is necessary, as can be seen by considering geodesics which enter a half-plane in $\mathbb{R}^{2}$ almost parallel to the boundary.

Proof Let $\epsilon>0$ and assume that $\xi_{0}, C, \gamma, \gamma^{\prime}, x, x^{\prime}$ are as in the statement. We may assume that $x \neq x^{\prime}$. In particular $\xi_{0} \notin C$. Let $p^{\prime}$ be the point of $\gamma^{\prime}$ the closest to $x$. Let $\left[x^{\prime}, y^{\prime}\right]$ be the intersection of $\gamma^{\prime}$ with $\mathcal{N}_{\epsilon} C$ (or $\left[x^{\prime}, y^{\prime}\left[\right.\right.$ with $y^{\prime} \in \partial_{\infty} X$ if $\gamma^{\prime} \cap \mathcal{N}_{\epsilon} C$ is unbounded). By assumption, $d\left(x^{\prime}, y^{\prime}\right) \geq c_{0}(\epsilon)$.

Case 1 Assume first that $p^{\prime}$ does not belong to $\left[\xi_{0}, x^{\prime}\right]$. If $d\left(x^{\prime}, p^{\prime}\right) \leq \frac{\epsilon}{2} c^{\prime \prime}(\epsilon)$, then let $a_{0}$ be the point $p^{\prime}$. Otherwise let $a_{0}$ be the point in $\left[x^{\prime}, y^{\prime}[\right.$ at distance $\frac{\epsilon}{2} c^{\prime \prime}(\epsilon)$ from $x^{\prime}$. This point exists and is at distance at least $\frac{\epsilon}{2} c^{\prime \prime}(\epsilon) \geq d\left(a_{0}, x^{\prime}\right)$ from $y^{\prime}$, as $d\left(x^{\prime}, y^{\prime}\right) \geq c_{0}(\epsilon) \geq \epsilon c^{\prime \prime}(\epsilon)$ by Equation (2). By Lemma 2.4, we have $d\left(a_{0}, C\right) \leq \epsilon-\left(1 / c^{\prime \prime}(\epsilon)\right) d\left(a_{0}, x^{\prime}\right)$.

Hence, if $a_{0}=p^{\prime}$, then

$$
\begin{aligned}
& \frac{1}{c^{\prime \prime}(\epsilon)} d\left(p^{\prime}, x^{\prime}\right)=\frac{1}{c^{\prime \prime}(\epsilon)} d\left(a_{0}, x^{\prime}\right) \leq \epsilon-d\left(a_{0}, C\right)=d(x, C)-d\left(p^{\prime}, C\right) \leq d\left(x, p^{\prime}\right) . \\
& \text { Thus, } \quad d\left(x, x^{\prime}\right) \leq d\left(x, p^{\prime}\right)+d\left(p^{\prime}, x^{\prime}\right) \leq\left(1+c^{\prime \prime}(\epsilon)\right) d\left(x, p^{\prime}\right),
\end{aligned}
$$

which proves the result, by the definition of $c_{2}^{\prime}(\epsilon)$.

If $a_{0} \neq p^{\prime}$, then $p^{\prime} \notin\left[a_{0}, \xi_{0}\right.$ [. Let us prove that $d\left(x, p^{\prime}\right) \geq \epsilon / 2$. This implies, by Lemma 2.3, that

$$
d\left(x, x^{\prime}\right) \leq c_{1}^{\prime}(\epsilon) \leq \frac{2 c_{1}^{\prime}(\epsilon)}{\epsilon} d\left(p^{\prime}, x\right)
$$


which proves the result, by the definition of $c_{2}^{\prime}(\epsilon)$. Let $b_{0}$ be the point in $\left[x^{\prime}, y^{\prime}\right]$ at distance $\frac{\epsilon}{2} c^{\prime \prime}(\epsilon)$ from $y^{\prime}$ (or $b_{0}=y^{\prime}$ if $y^{\prime}$ is at infinity). By Lemma 2.4, we have

$$
\max \left\{d\left(a_{0}, C\right), d\left(b_{0}, C\right)\right\} \leq \epsilon-\frac{1}{c^{\prime \prime}(\epsilon)} \min \left\{d\left(a_{0}, x^{\prime}\right), d\left(b_{0}, y^{\prime}\right)\right\}=\frac{\epsilon}{2} .
$$

Assume by contradiction that $d\left(x, p^{\prime}\right)<\frac{\epsilon}{2}$. If $p^{\prime} \in\left[a_{0}, b_{0}\right]$, then by convexity $d\left(p^{\prime}, C\right) \leq \frac{\epsilon}{2}$, therefore $d(x, C) \leq d\left(x, p^{\prime}\right)+d\left(p^{\prime}, C\right)<\epsilon$, a contradiction. If otherwise $p^{\prime} \notin\left[a_{0}, b_{0}\right]$, then $b_{0} \in\left[p^{\prime}, \xi_{0}[\right.$. Therefore there exists a point $z$ in $\left[x, \xi_{0}\right.$ [ whose closest point to $\left[p^{\prime}, \xi_{0}\left[\right.\right.$ is $b_{0}$. By convexity, $d\left(z, b_{0}\right)<\frac{\epsilon}{2}$. Hence $d(z, C) \leq d\left(z, b_{0}\right)+d\left(b_{0}, C\right)<\epsilon$, which contradicts the fact that $\gamma$ enters $\mathcal{N}_{\epsilon} C$ at $x$.

Case 2 Assume now that $p^{\prime}$ belongs to $\left[\xi_{0}, x^{\prime}\right]$. Let $a^{\prime}$ and $b^{\prime}$ be the points of $C$ the closest to $x^{\prime}$ and $y^{\prime}$ respectively. They are at distance $\epsilon>0$ from $x^{\prime}$ and $y^{\prime}$ respectively (except that $b^{\prime}=y^{\prime}$ if $y^{\prime}$ is at infinity). Let $\phi$ be the comparison angle at $x^{\prime}$ between the geodesic segments $\left[x^{\prime}, a^{\prime}\right]$ and $\left[x^{\prime}, y^{\prime}[\right.$. We claim that $\sin \phi \leq 1 / \sqrt{\cosh \epsilon}$.

To prove this claim, if $y^{\prime} \in X$, we construct a comparison quadrilateral with vertices $\overline{x^{\prime}}, \overline{a^{\prime}}, \overline{b^{\prime}}, \overline{y^{\prime}} \in \mathbb{H}_{\mathbb{R}}^{2}$ by gluing together the comparison triangles $\left(\overline{x^{\prime}}, \overline{a^{\prime}}, \overline{y^{\prime}}\right)$ of $\left(x^{\prime}, a^{\prime}, y^{\prime}\right)$ and $\left(\overline{a^{\prime}}, \overline{b^{\prime}}, \overline{y^{\prime}}\right)$ of $\left(a^{\prime}, b^{\prime}, y^{\prime}\right)$ along their isometric edges $\left[\overline{a^{\prime}}, \overline{y^{\prime}}\right]$. If $y^{\prime} \notin X$, then $b^{\prime}=y^{\prime}$, and the above quadrilateral is replaced by the comparison triangle with vertices $\overline{x^{\prime}}, \overline{a^{\prime}}, \overline{y^{\prime}} \in \mathbb{H}_{\mathbb{R}}^{2} \cup\{\infty\}$. By comparison, all angles in the quadrilateral in $\mathbb{H}_{\mathbb{R}}^{2}$ with vertices $\overline{x^{\prime}}, \overline{a^{\prime}}, \overline{b^{\prime}}, \overline{y^{\prime}}$ are greater than or equal to those in the quadrilateral in $X$ with vertices $x^{\prime}, a^{\prime}, b^{\prime}, y^{\prime}$. In particular, if the angle at $\overline{x^{\prime}}$ is $\bar{\phi}$, we have $\phi \leq \bar{\phi}$. If the quadrilateral with vertices $\overline{x^{\prime}}, \overline{a^{\prime}}, \overline{b^{\prime}}, \overline{y^{\prime}}$ is replaced by the one with vertices $\overline{x^{\prime}}, \overline{a^{\prime}} *, \overline{b^{\prime}} *, \overline{y^{\prime}}$ with $d\left(\overline{x^{\prime}}, \overline{a^{\prime}} *\right)=\epsilon=d\left(\overline{y^{\prime}}, \overline{b^{\prime}} *\right)$ and right angles at $\overline{a^{\prime}} *$ and $\bar{b}_{*}^{\prime}$, the angle $\bar{\phi}_{*}$ at $\overline{x^{\prime}}$ of this quadrilateral is at least $\bar{\phi}$. Furthermore, this quadrilateral is symmetric: the angle at $\overline{y^{\prime}}$ is also $\bar{\phi}_{*}$. Thus, we get an upper bound for $\phi$ by estimating $\bar{\phi}_{*}$.

Let $\left[m, m^{\prime}\right]$ be the common perpendicular segment between $\left[\overline{x^{\prime}}, \overline{y^{\prime}}\right]$ and $\left[\overline{a^{\prime}} *, \overline{b^{\prime}} *\right]$, with $m \in\left[\overline{x^{\prime}}, \overline{y^{\prime}}\right]$. We have by for instance Beardon [5, page 157]

$$
\sin \bar{\phi}_{*}=\frac{\cosh d\left(m, m^{\prime}\right)}{\cosh \epsilon} \text { and } \cosh d\left(\overline{x^{\prime}}, m\right)=\frac{\sinh \epsilon}{\sinh d\left(m, m^{\prime}\right)} .
$$

Hence, as $d\left(x^{\prime}, y^{\prime}\right) \geq c_{0}(\epsilon)$,

$$
\begin{aligned}
\sin \bar{\phi}_{*} & =\frac{\sqrt{1+\left(\sinh ^{2} \epsilon\right) /\left(\cosh ^{2} d\left(\overline{x^{\prime}}, m\right)\right)}}{\cosh \epsilon} \\
& \leq \frac{\sqrt{1+\left(\sinh ^{2} \epsilon\right) /\left(\cosh ^{2}\left(c_{0}(\epsilon) / 2\right)\right)}}{\cosh \epsilon} \leq \frac{1}{\sqrt{\cosh \epsilon}},
\end{aligned}
$$


as, by Equation (2), $c_{0}(\epsilon) \geq \epsilon c^{\prime \prime}(\epsilon) \geq 2 \operatorname{arcosh}(\sqrt{2} \cosh (\epsilon / 2))$. This proves the claim. By convexity, the comparison angle at $x^{\prime}$ between the geodesic segments $\left[x^{\prime}, x\right]$ and $\left[x^{\prime}, a^{\prime}\right]$ is at most $\frac{\pi}{2}$. Hence the comparison angle $\theta$ at $x^{\prime}$ between $\left[x^{\prime}, x\right]$ and $\left[x^{\prime}, \xi_{0}[\right.$ (which lies in $\left[0, \frac{\pi}{2}\left[\right.\right.$ since $p^{\prime} \in\left[\xi_{0}, x^{\prime}\left[\right.\right.$ ) is at least $\pi-\frac{\pi}{2}-\phi=\frac{\pi}{2}-\phi$. In particular,

$$
\frac{1}{\sin \theta} \leq \frac{1}{\sin \left(\frac{\pi}{2}-\phi\right)}=\frac{1}{\sqrt{1-\sin ^{2} \phi}} \leq \sqrt{\frac{\cosh \epsilon}{\cosh \epsilon-1}} .
$$

If $p^{\prime}=x^{\prime}$, then the result holds, since $c_{2}^{\prime}(\epsilon) \geq 1$. Otherwise, since $\gamma^{\prime}$ enters $\mathcal{N}_{\epsilon} C$ at $x^{\prime}$, the points $x, x^{\prime}, p^{\prime}$ are pairwise distinct. Let $\left(\bar{x}, \overline{x^{\prime}}, \overline{p^{\prime}}\right)$ be a comparison triangle in $\mathbb{H}_{\mathbb{R}}^{2}$ of the geodesic triangle $\left(x, x^{\prime}, p^{\prime}\right)$. By comparison, $\bar{\theta}=\angle_{\overline{x^{\prime}}}\left(\bar{x}, \overline{p^{\prime}}\right) \geq \theta$ and $\overline{p^{\prime}}$ is the closest point to $\bar{x}$ on $\left[\overline{p^{\prime}}, \overline{x^{\prime}}\right]$. In particular, the angle $\angle \overline{p^{\prime}}\left(\bar{x}, \overline{x^{\prime}}\right)$ is at least $\frac{\pi}{2}$. By the formulae in right-angled hyperbolic triangles, we have

$$
\frac{\sinh d\left(x, p^{\prime}\right)}{\sinh d\left(x, x^{\prime}\right)}=\frac{\sinh d\left(\bar{x}, \overline{p^{\prime}}\right)}{\sinh d\left(\bar{x}, \overline{x^{\prime}}\right)} \geq \sin \bar{\theta} \geq \sin \theta .
$$

Since $p^{\prime}$ is the closest point to $x$ on $\gamma^{\prime}$, we have $d\left(x, p^{\prime}\right) \leq d\left(x, x^{\prime}\right) \leq c_{1}^{\prime}(\epsilon)$. In particular, by the convexity of the map $t \mapsto \sinh t$ on $[0,+\infty[$, we have

$$
\sinh d\left(x, p^{\prime}\right) \leq \frac{\sinh c_{1}^{\prime}(\epsilon)}{c_{1}^{\prime}(\epsilon)} d\left(x, p^{\prime}\right) .
$$

Hence, by the definition of $c_{2}^{\prime}(\epsilon)$,

$$
d\left(x, x^{\prime}\right) \leq \sinh d\left(x, x^{\prime}\right) \leq \frac{\sinh d\left(x, p^{\prime}\right)}{\sin \theta} \leq c_{2}^{\prime}(\epsilon) d\left(x, p^{\prime}\right) .
$$

In general, there is no estimate analogous to Lemma 2.5 for the distance between the points $y, y^{\prime}$ where two geodesic rays or lines $\gamma, \gamma^{\prime}$ starting from a point $\xi_{0}$ exit an $\epsilon$-convex subset $\mathcal{N}_{\epsilon} C$. For instance, the geodesic line $\gamma$ could be tangent to $\mathcal{N}_{\epsilon} C$, and $\gamma^{\prime}$ could enter for a long time in $\mathcal{N}_{\epsilon} C$, so that $y$ and $y^{\prime}$ would not be close. But the result is not true even if we assume that both $\gamma$ and $\gamma^{\prime}$ meet $\mathcal{N}_{\epsilon} C$ in a long segment. Here is a counterexample when $X$ is a tree (but this phenomenon is not specific to trees).

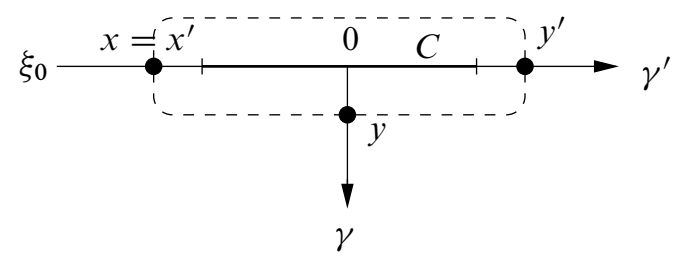


Let $\gamma, \gamma^{\prime}$ be two geodesic lines in a tree $X$, coinciding on their negative subrays, starting at $\xi_{0} \in \partial_{\infty} X$, and with disjoint positive subrays. Let $\epsilon=\eta=1$, and $C=\gamma^{\prime}([-\ell,+\ell])$. Then the entering points of $\gamma, \gamma^{\prime}$ in $\mathcal{N}_{\epsilon} C$ are $x=x^{\prime}=\gamma^{\prime}(-\ell-1)$. Besides, $y=\gamma(1)$, $y^{\prime}=\gamma^{\prime}(\ell+1)$ and $d\left(y, \gamma^{\prime}\right) \leq 1$. But we have $d\left(y, y^{\prime}\right)=\ell+2$, which goes to $+\infty$ as $\ell \rightarrow+\infty$.

This explains the dichotomy in the following result on the exiting points from an $\epsilon$-convex sets of two geodesic lines which start from the same point at infinity. For every $\epsilon, \eta>0$, we define

$$
\begin{gathered}
h^{\prime}(\epsilon, \eta)=\max \left\{2 \eta+\max \left\{0,-2 \log \frac{\epsilon}{2}\right\}, \eta+c_{1}^{\prime}(\epsilon)+c_{0}(\epsilon)\right\}, \\
c_{3}^{\prime}(\epsilon)=3+\frac{2 c_{1}^{\prime}(\epsilon)}{\epsilon} .
\end{gathered}
$$

Lemma 2.6 Let $\epsilon, \eta>0$. Let $C$ be a convex subset in $X, \xi_{0} \in X \cup \partial_{\infty} X$, and $\gamma, \gamma^{\prime}$ geodesic rays or lines starting from $\xi_{0}$. If $\gamma$ enters $\mathcal{N}_{\epsilon} C$ at a point $x \in X$ and exits $\mathcal{N}_{\epsilon} C$ at a point $y \in X$ such that $d(x, y) \geq h^{\prime}(\epsilon, \eta)$ and $d\left(y, \gamma^{\prime}\right) \leq \eta$, then $\gamma^{\prime}$ meets $\mathcal{N}_{\epsilon} C$, entering it at a point $x^{\prime} \in X$, exiting it at a point $y^{\prime} \in X \cup \partial_{\infty} X$ such that

$$
d\left(y, y^{\prime}\right) \leq c_{3}^{\prime}(\epsilon) d\left(y, \gamma^{\prime}\right) \quad \text { or } \quad d\left(x^{\prime}, y^{\prime}\right)>d(x, y) .
$$

Proof Let $p^{\prime}$ be the closest point on $\gamma^{\prime}$ to $y$. Let $q$ be the closest point on $\gamma$ to $p^{\prime}$. The point $q$ belongs to $\left[y, \xi_{0}\right]$ and satisfies $d(y, q) \leq d\left(y, p^{\prime}\right) \leq \eta$, as closest point maps do not increase the distances. By the properties of geodesic triangles in CAT $(-1)$ spaces, we have

$$
d\left(p^{\prime}, q\right) \leq \operatorname{arsinh} 1=\log (1+\sqrt{2}) .
$$

Let us first prove that $\gamma^{\prime}$ meets $\mathcal{N}_{\epsilon} C$. Let $m$ be the midpoint of $[x, y]$. As

$$
d(y, m)=d(x, y) / 2 \geq h^{\prime}(\epsilon, \eta) / 2 \geq \eta \geq d(y, q),
$$

the point $q$ belongs to $[m, y]$. Furthermore,

$$
d(q, m)=d(y, m)-d(y, q) \geq h^{\prime}(\epsilon, \eta) / 2-\eta \geq-\log \frac{\epsilon}{2},
$$

by the definition of $h^{\prime}(\epsilon, \eta)$. By Lemma 2.1, we have

$$
d\left(m, \gamma^{\prime}\right) \leq e^{-d(q, m)} \sinh d\left(q, p^{\prime}\right) \leq \frac{\epsilon}{2} .
$$

By Lemma 2.2, as $d(x, y) \geq h^{\prime}(\epsilon, \eta) \geq c_{0}(\epsilon)$ by the definition of $h^{\prime}(\epsilon, \eta)$, we have $d(m, C) \leq \frac{\epsilon}{2}$. Hence the point $m^{\prime}$ of $\gamma^{\prime}$ the closest to $m$ belongs to $\mathcal{N}_{\epsilon} C$, which is what we wanted. 
Let $x^{\prime}$ and $y^{\prime}$ be the entering point in $\mathcal{N}_{\epsilon} C$ and exiting point out of $\mathcal{N}_{\epsilon} C$ of $\gamma^{\prime}$ respectively. The point $y^{\prime}$ could for the moment be at infinity, in which case the second possibility below would hold.

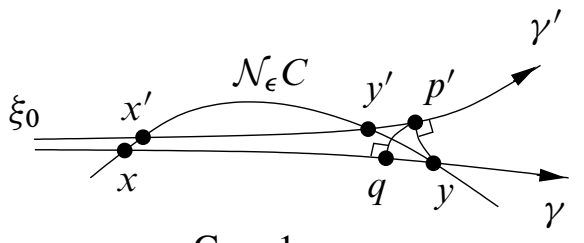

Case 1

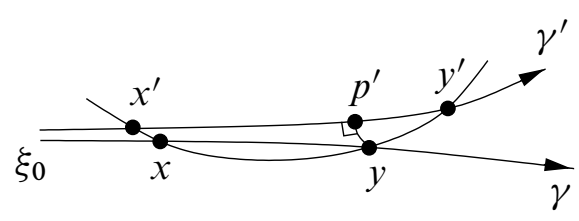

Case 2

Case 1 Assume that $p^{\prime} \notin\left[y^{\prime}, \xi_{0}\right]$. Let $\eta_{\epsilon}=\epsilon c^{\prime \prime}(\epsilon) / 2$. There are two subcases. First assume that $d\left(y, p^{\prime}\right) \geq \epsilon / 2$. Let

$$
t_{\epsilon}=\max \left\{\eta_{\epsilon},-\log \frac{\epsilon}{2}\right\} .
$$

Note that $h^{\prime}(\epsilon, \eta) \geq \eta_{\epsilon}+t_{\epsilon}+\eta$ by the definition of $h^{\prime}(\epsilon, \eta)$, as by Equation (2) we have $c_{0}(\epsilon) \geq \epsilon c^{\prime \prime}(\epsilon)=2 \eta_{\epsilon}$, and by a discussion on the value of $t_{\epsilon}$. Hence we have

$$
d(y, x)-d(y, q)-t_{\epsilon} \geq h^{\prime}(\epsilon, \eta)-\eta-t_{\epsilon} \geq \eta_{\epsilon} \geq 0 .
$$

Therefore, the point $y_{0}$ in $[x, q]$ at distance $t_{\epsilon}$ of $q$ exists and satisfies $d\left(y_{0}, x\right) \geq \eta_{\epsilon}$. Furthermore, $d\left(y_{0}, q\right)=t_{\epsilon} \geq \eta_{\epsilon}$ and

$$
d(x, q)=d(x, y)-d(y, q) \geq h^{\prime}(\epsilon, \eta)-\eta \geq c_{0}(\epsilon) \geq 2 \eta_{\epsilon},
$$

by the definition of $h^{\prime}(\epsilon, \eta)$. Let $a_{0}$ and $b_{0}$ be the points in $[x, q]$ at distance $\eta_{\epsilon}$ from $x$ and $q$ respectively, which are at distance at least $\eta_{\epsilon}$ from $q$ and $x$ respectively. By Lemma 2.4, we have $d\left(a_{0}, C\right) \leq \epsilon-\eta_{\epsilon} / c^{\prime \prime}(\epsilon)=\epsilon / 2$, and similarly $d\left(b_{0}, C\right) \leq \epsilon / 2$. Note that $y_{0}$ belongs to $\left[a_{0}, b_{0}\right]$. Hence by convexity, we have $d\left(y_{0}, C\right) \leq \epsilon / 2$. By Lemma 2.1, we have

$$
d\left(y_{0}, \gamma^{\prime}\right) \leq e^{-t_{\epsilon}} \sinh d\left(q, p^{\prime}\right) \leq \frac{\epsilon}{2}
$$

Therefore the point $q^{\prime}$ on $\gamma^{\prime}$ the closest to $y_{0}$ belongs to $\mathcal{N}_{\epsilon} C$. As $y^{\prime}$ is the exiting point of $\gamma^{\prime}$ from $\mathcal{N}_{\epsilon} C$, it belongs to $\left[q^{\prime}, p^{\prime}\right]$. As closest point maps do not increase the distances, we have $d\left(p^{\prime}, q^{\prime}\right) \leq d\left(y, y_{0}\right)$. Hence

$$
\begin{aligned}
d\left(y, y^{\prime}\right) & \leq d\left(y, p^{\prime}\right)+d\left(p^{\prime}, y^{\prime}\right) \leq d\left(y, p^{\prime}\right)+d\left(p^{\prime}, q^{\prime}\right) \leq d\left(y, p^{\prime}\right)+d\left(y, y_{0}\right) \\
& \leq d\left(y, p^{\prime}\right)+d(y, q)+d\left(q, y_{0}\right) \leq 2 d\left(y, p^{\prime}\right)+t_{\epsilon} \\
& \leq\left(2+2 t_{\epsilon} / \epsilon\right) d\left(y, p^{\prime}\right) \leq c_{3}^{\prime}(\epsilon) d\left(y, p^{\prime}\right),
\end{aligned}
$$

as it can be checked that $2 c_{1}^{\prime}(\epsilon) / \epsilon+1 \geq 2 t_{\epsilon} / \epsilon$. 
Assume now that $d\left(y, p^{\prime}\right) \leq \epsilon / 2$. Since

$$
d(x, y) \geq h^{\prime}(\epsilon, \eta) \geq c_{0}(\epsilon) \geq 2 \eta_{\epsilon} \geq 2 c^{\prime \prime}(\epsilon) d\left(y, p^{\prime}\right),
$$

the point $y_{0}$ in $[x, y]$ at distance $c^{\prime \prime}(\epsilon) d\left(y, p^{\prime}\right)$ from $y$ exists and is at distance at least $c^{\prime \prime}(\epsilon) d\left(y, p^{\prime}\right)$ from $x$. Hence by Lemma 2.4, we have $d\left(y_{0}, C\right) \leq \epsilon-d\left(y, p^{\prime}\right)$. Let $q^{\prime}$ be the point on $\gamma^{\prime}$ the closest to $y_{0}$. By convexity, $q^{\prime}$ is at distance at most $d\left(y, p^{\prime}\right)$ from $y_{0}$, hence belongs to $\mathcal{N}_{\epsilon} C$. As $y^{\prime}$ is the exiting point of $\gamma^{\prime}$ from $\mathcal{N}_{\epsilon} C$, it belongs to $\left[q^{\prime}, p^{\prime}\right]$. As closest point maps do not increase distances, we have $d\left(q^{\prime}, p^{\prime}\right) \leq d\left(y_{0}, y\right)$. Hence, as above, and by the definition of $y_{0}$,

$$
d\left(y, y^{\prime}\right) \leq d\left(y, p^{\prime}\right)+d\left(y_{0}, y\right) \leq\left(1+c^{\prime \prime}(\epsilon)\right) d\left(y, p^{\prime}\right),
$$

which proves the result, by the definition of $c_{3}^{\prime}(\epsilon)$, as $2 c_{1}^{\prime}(\epsilon) / \epsilon+1 \geq c^{\prime \prime}(\epsilon)$.

Case 2 Assume that $\left.\left.p^{\prime} \in\right] y^{\prime}, \xi_{0}\right]$. Lemma 2.3 implies that $d\left(x, x^{\prime}\right) \leq c_{1}^{\prime}(\epsilon)$. Note that $p^{\prime} \notin\left[x^{\prime}, \xi_{0}\right]$. Otherwise, with $q$ and $s$ the closest points to $p^{\prime}$ and $x^{\prime}$ on $\gamma$ respectively, we would have $\left.s \notin] q, \xi_{0}\right]$ by convexity. As $q \in\left[y, \xi_{0}\right]$, we would then have

$$
d(x, y) \leq d(x, s)+d(q, y) \leq d\left(x, x^{\prime}\right)+d\left(p^{\prime}, y\right) \leq c_{1}^{\prime}(\epsilon)+\eta<h^{\prime}(\epsilon, \eta),
$$

by the definition of $h^{\prime}(\epsilon, \eta)$, a contradiction.

Assume first that $d\left(y, p^{\prime}\right)<\epsilon / 2$. We start by observing that $d\left(p^{\prime}, y^{\prime}\right) \leq c^{\prime \prime}(\epsilon) d\left(y, p^{\prime}\right)$. Indeed, suppose by contradiction that $d\left(p^{\prime}, y^{\prime}\right)>c^{\prime \prime}(\epsilon) d\left(y, p^{\prime}\right)$. By continuity of the closest point maps, let $y_{0}$ be a point on $\gamma$ that does not belong to $\mathcal{N}_{\epsilon} C$, but is close enough to $y$, so that the closest point $q^{\prime}$ to $y_{0}$ on $\gamma^{\prime}$ belongs to $\left[p^{\prime}, y^{\prime}\right]$ and satisfies $d\left(y_{0}, q^{\prime}\right) \leq \epsilon / 2$ and $d\left(q^{\prime}, y^{\prime}\right) \geq c^{\prime \prime}(\epsilon) d\left(q^{\prime}, y_{0}\right)$. Hence, using the definition of $h^{\prime}(\epsilon, \eta)$ and Equation (2), we have

$$
\begin{aligned}
d\left(y^{\prime}, x^{\prime}\right) & \geq d\left(q^{\prime}, x^{\prime}\right) \geq d\left(p^{\prime}, x^{\prime}\right) \geq d(x, y)-d\left(p^{\prime}, y\right)-d\left(x, x^{\prime}\right) \\
& \geq h^{\prime}(\epsilon, \eta)-\eta-c_{1}^{\prime}(\epsilon) \geq c_{0}(\epsilon) \geq \epsilon c^{\prime \prime}(\epsilon) \geq 2 c^{\prime \prime}(\epsilon) d\left(q^{\prime}, y_{0}\right) .
\end{aligned}
$$

Let $a_{0}$ and $b_{0}$ be the points in $\left[x^{\prime}, y^{\prime}\right]$ at distance $c^{\prime \prime}(\epsilon) d\left(q^{\prime}, y_{0}\right) \leq \epsilon c^{\prime \prime}(\epsilon) / 2$ from $x^{\prime}$ and $y^{\prime}$ respectively. The estimate (7) implies that $a_{0}$ and $b_{0}$ are at distance at least $c^{\prime \prime}(\epsilon) d\left(q^{\prime}, y_{0}\right)$ from $y^{\prime}$ and $x^{\prime}$ respectively. By Lemma 2.4, we have $d\left(a_{0}, C\right) \leq$ $\epsilon-d\left(q^{\prime}, y_{0}\right)$ and $d\left(b_{0}, C\right) \leq \epsilon-d\left(q^{\prime}, y_{0}\right)$. Hence, the point $q^{\prime}$, which belongs to $\left[a_{0}, b_{0}\right]$ by Formula (7) and the construction of $q^{\prime}$, is by convexity at distance at most $\epsilon-d\left(q^{\prime}, y_{0}\right)$ from $C$. Therefore by the triangle inequality, $d\left(y_{0}, C\right) \leq \epsilon$, which is a contradiction. Hence $d\left(p^{\prime}, y^{\prime}\right) \leq c^{\prime \prime}(\epsilon) d\left(y, p^{\prime}\right)$, and

$$
d\left(y, y^{\prime}\right) \leq d\left(y, p^{\prime}\right)+d\left(p^{\prime}, y^{\prime}\right) \leq\left(1+c^{\prime \prime}(\epsilon)\right) d\left(y, p^{\prime}\right),
$$

which proves the result, as in the end of Case 1. 
Assume now that $d\left(y, p^{\prime}\right) \geq \epsilon / 2$. Suppose first that $d\left(p^{\prime}, y^{\prime}\right)>d\left(y, p^{\prime}\right)+c_{1}^{\prime}(\epsilon)$. Then, as $p^{\prime} \in\left[x^{\prime}, y^{\prime}\right]$,

$d\left(x^{\prime}, y^{\prime}\right)=d\left(x^{\prime}, p^{\prime}\right)+d\left(p^{\prime}, y^{\prime}\right) \geq d\left(p^{\prime}, y^{\prime}\right)+d(x, y)-d\left(y, p^{\prime}\right)-d\left(x, x^{\prime}\right)>d(x, y)$, which is one of the two possible conclusions. Otherwise,

$$
\begin{aligned}
d\left(y, y^{\prime}\right) \leq d\left(y, p^{\prime}\right)+d\left(p^{\prime}, y^{\prime}\right) & \leq 2 d\left(y, p^{\prime}\right)+c_{1}^{\prime}(\epsilon) \\
& \leq\left(2+\frac{2 c_{1}^{\prime}(\epsilon)}{\epsilon}\right) d\left(y, p^{\prime}\right) \leq c_{3}^{\prime}(\epsilon) d\left(y, p^{\prime}\right),
\end{aligned}
$$

by the definition of $c_{3}^{\prime}(\epsilon)$. This is the other possible conclusion.

Remark 2.7 The asymptotic behaviour of the constants when $\epsilon$ is very big or very small is as follows.

- $c_{0}(\epsilon) \sim 3 \epsilon$ as $\epsilon \rightarrow+\infty$ and $\lim _{\epsilon \rightarrow 0} c_{0}(\epsilon)=4 \log 2 \approx 2.77$.

- $\lim _{\epsilon \rightarrow+\infty} c_{1}^{\prime}(\epsilon)=c_{1}^{\prime}(\infty)=2 \log (1+\sqrt{2}) \approx 1.76$, and $c_{1}^{\prime}(\epsilon) \sim-2 \log \epsilon$ as $\epsilon \rightarrow 0$. Note that $\epsilon \mapsto c_{1}^{\prime}(\epsilon)$ is decreasing.

- $\lim _{\epsilon \rightarrow+\infty} c^{\prime \prime}(\epsilon)=1$, and $c^{\prime \prime}(\epsilon) \sim \frac{2}{\epsilon} \log (2+\sqrt{3})$ as $\epsilon \rightarrow 0$.

- For $\epsilon \operatorname{big}, c_{2}^{\prime}(\epsilon)=c^{\prime \prime}(\epsilon)+1$, hence $\lim _{\epsilon \rightarrow+\infty} c_{2}^{\prime}(\epsilon)=2$. For $\epsilon>0$ small,

$$
c_{2}^{\prime}(\epsilon)=\sqrt{\frac{\cosh \epsilon}{\cosh \epsilon-1}} \frac{\sinh c_{1}^{\prime}(\epsilon)}{c_{1}^{\prime}(\epsilon)} \sim \frac{\sqrt{2}}{4 \epsilon^{3} \log (1 / \epsilon)} .
$$

- $\lim _{\epsilon \rightarrow+\infty} c_{3}^{\prime}(\epsilon)=3$, and $c_{3}^{\prime}(\epsilon) \sim-\frac{4}{\epsilon} \log \epsilon$ as $\epsilon \rightarrow 0$.

- $h^{\prime}(\epsilon, \eta) \sim 3 \epsilon$ as $\epsilon \rightarrow+\infty$, and $h^{\prime}(\epsilon, \eta) \sim-2 \log \epsilon$ as $\epsilon \rightarrow 0$, uniformly on compact subsets of $\eta$ 's.

When $\epsilon$ goes to $+\infty, c_{1}^{\prime}(\epsilon)$ and $c_{3}^{\prime}(\epsilon)$ have finite limits, and the limiting values apply for the horoball case; see Lemmas 2.9 and 2.12 below. On the other hand, the constants $c_{0}(\epsilon)$ and $h^{\prime}(\epsilon, \eta)$ behave badly as $\epsilon \rightarrow \infty$, and we will improve them in Section 2.3.

When $X$ is a tree, the constants $c_{3}^{\prime}(\epsilon)$ and $h^{\prime}(\epsilon, \eta)$ can be simplified, we can take $c_{3}^{\prime}(\epsilon)=2$ and any $h^{\prime}(\epsilon, \eta)>2 \eta$, as the following more precise result shows, improving Lemma 2.6 for trees. Note that the versions of Lemma 2.3 and Lemma 2.5 for trees simply say that we can take $c_{0}(\epsilon)=\epsilon$, and $c_{1}^{\prime}(\epsilon)=c_{2}^{\prime}(\epsilon)=0$, since for every point or end $\xi_{0}$ of a (real) tree, for every convex subset $C$, for all geodesic rays or lines $\gamma, \gamma^{\prime \prime}$ starting from $\xi_{0}$ and entering $C$ in $x, x^{\prime}$ respectively, we have $x=x^{\prime}$. 
Remark 2.8 Let $X$ be an $\mathbb{R}$-tree and $\epsilon>0$. Let $C$ be a convex subset in $X$, $\xi_{0} \in X \cup \partial_{\infty} X$, and $\gamma, \gamma^{\prime}$ geodesic rays or lines starting from $\xi_{0}$. If $\gamma$ enters $\mathcal{N}_{\epsilon} C$ at a point $x \in X$ and exits $\mathcal{N}_{\epsilon} C$ at a point $y \in X$ such that $d(x, y)>2 d\left(y, \gamma^{\prime}\right)$, then $\gamma^{\prime}$ meets $\mathcal{N}_{\epsilon} C$, entering it at $x^{\prime}=x$, exiting it at a point $y^{\prime}$ (possibly at infinity) such that

$$
d\left(y, y^{\prime}\right) \leq 2 d\left(y, \gamma^{\prime}\right) \quad \text { or } \quad d\left(x^{\prime}, y^{\prime}\right)>d(x, y)
$$

Proof Let $p^{\prime}$ be the closest point to $y$ on $\gamma^{\prime}$. Note that $p^{\prime}$ belongs to $] \xi_{0}, y$ ], as $X$ is a tree and $\gamma^{\prime}$ also starts from $\xi_{0}$. If $\left.p^{\prime} \in\right] \xi_{0}, x\left[\right.$, then $d\left(y, \gamma^{\prime}\right)>d(x, y)$, a contradiction. Hence $p^{\prime} \in[x, y] \subset \mathcal{N}_{\epsilon} C$, and $\gamma^{\prime}$ enters $\mathcal{N}_{\epsilon} C$ at $x^{\prime}=x$.

Suppose first that $d(x, y)<2 \epsilon$. Then the closest point $z$ to $y$ in $C$ does not belong to $[x, y]$. Let $q$ be the midpoint of $[x, y]$, which is also the closest point to $z$ on $[x, y]$. As $d(x, y)>2 d\left(y, \gamma^{\prime}\right)$, the point $p^{\prime}$ belongs to $\left.] q, y\right]$, hence $d\left(y, y^{\prime}\right)=2 d\left(y, \gamma^{\prime}\right)$, which is fine.

Assume now that $d(x, y) \geq 2 \epsilon$. If $x_{\epsilon}$ and $y_{\epsilon}$ are the points in $[x, y]$ at distance $\epsilon$ from $x$ and $y$ respectively, then $[x, y] \cap C=\left[x_{\epsilon}, y_{\epsilon}\right]$. If $p^{\prime}$ belongs to $\left.] y_{\epsilon}, y\right]$, then $y_{\epsilon}$ is also the closest point to $y^{\prime}$ in $C$, and $d\left(p^{\prime}, y\right)=d\left(p^{\prime}, y^{\prime}\right)$, so that $d\left(y, y^{\prime}\right)=$ $2 d\left(y, \gamma^{\prime}\right)$, which is fine. Otherwise, we have $d\left(y, \gamma^{\prime}\right) \geq \epsilon$. If $d\left(x^{\prime}, y^{\prime}\right) \leq d(x, y)$, then $d\left(p^{\prime}, y^{\prime}\right) \leq d\left(p^{\prime}, y\right)$. Hence

$$
d\left(y, y^{\prime}\right)=d\left(y, p^{\prime}\right)+d\left(p^{\prime}, y^{\prime}\right) \leq 2 d\left(y, p^{\prime}\right)=2 d\left(y, \gamma^{\prime}\right) .
$$

\subsection{Hitting horoballs}

As shown in Remark 2.7, the constants $c_{0}(\epsilon)$ and $h^{\prime}(\epsilon, \eta)$, used to describe the penetration of geodesic lines inside $\epsilon$-convex subsets, do not have a finite limit as $\epsilon$ goes to $+\infty$. Horoballs are $\epsilon$-convex subsets for every $\epsilon$, and we could use for instance $\epsilon=1$ in these constants to get numerical values. But in order to get better values, we will prove analogs for horoballs of Lemmas 2.3, 2.4, 2.5 and 2.6. The proofs of the lemmas below follow the same lines as the ones for the general case of $\epsilon$-convex subsets given in Section 2.2, with many simplifications.

As $c_{1}^{\prime}(\epsilon)$ tends to

$$
c_{1}^{\prime}(\infty)=2 \log (1+\sqrt{2})
$$

the next lemma follows by passing to the limit in Lemma 2.3. It is not hard to see (for instance by considering the real hyperbolic plane) that the constant $c_{1}^{\prime}(\infty)$ is optimal. 
Lemma 2.9 For every horoball $H$ in $X$, for every $\xi_{0}$ in $\left(X \cup \partial_{\infty} X\right)-(H \cup H[\infty])$, for all geodesic rays or lines $\gamma$ and $\gamma^{\prime}$ starting from $\xi_{0}$ and entering $H$ in $x$ and $x^{\prime}$ respectively, we have

$$
d\left(x, x^{\prime}\right) \leq c_{1}^{\prime}(\infty)=2 \log (1+\sqrt{2}) .
$$

The following result, Lemma 2.10, improves Lemma 2.4 for horoballs, and says that when the $\epsilon$-convex subset under consideration is a horoball, we can replace $c_{0}(\epsilon)$ by

$$
c_{0}(\infty)=4.056
$$

and $c^{\prime \prime}(\epsilon)$ by $c^{\prime \prime}(\infty)=\frac{3}{2}$. Lemma 2.11 below is the analog of Lemma 2.5 for horoballs, and says that when the $\epsilon$-convex subset under consideration is a horoball, we can replace $c_{0}(\epsilon)$ by $c_{0}(\infty)=4.056$ and $c_{2}^{\prime}(\epsilon)$ by

$$
c_{2}^{\prime}(\infty)=\frac{5}{2} \text {. }
$$

Note that $c^{\prime \prime}(\infty), c_{0}(\infty)$ and $c_{2}^{\prime}(\infty)$ are not limits as $\epsilon$ goes to $\infty$ of $c^{\prime \prime}(\epsilon), c_{0}(\epsilon)$ and $c_{2}^{\prime}(\epsilon)$, but this mnemonic notation will be useful in Section 4, where it will be used in a similar way whether $\epsilon$ is finite or not.

Lemma 2.10 For every horoball $H$, for every $a$ and $b$ in $\partial H$ with $d(a, b) \geq c_{0}(\infty)$, for every $a_{0}$ in $[a, b]$, we have

$$
a_{0} \in H\left[\frac{2}{3} \min \left\{d\left(a_{0}, a\right), d\left(a_{0}, b\right)\right\}\right] .
$$

Proof Let $\xi=H[\infty]$ be the point at infinity of $H$. Up to exchanging $a$ and $b$, we may assume that $\ell=d\left(a_{0}, a\right)=\min \left\{d\left(a_{0}, a\right), d\left(a_{0}, b\right)\right\}$.

Let $(\bar{a}, \bar{b}, \bar{\xi}=\infty)$ be a comparison triangle of $(a, b, \xi)$ in $\mathbb{H}_{\mathbb{R}}^{2}$. By comparison (see the paragraph before Lemma 2.1), the (nonnegative) difference $\ell^{\prime}$ of the heights of $a$ and $a_{0}$ with respect to $\xi$ is at least the corresponding quantity $\overline{\ell^{\prime}}$ for the comparison points $\bar{a}$ and $\bar{a}_{0}$. Thus, in order to show that $\ell^{\prime} \geq \frac{2}{3} \ell$, it is sufficient to show that $\overline{\ell^{\prime}} \geq \frac{2}{3} \ell$, and the question reduces to the case $X=\mathbb{H}_{\mathbb{R}}^{2}$. We assume that $[b, a]$ lies on the unit circle, with $a$ (and hence $a_{0}$, as $a$ and $b$ have the same (Euclidean) vertical coordinate) in the closed positive quadrant.

Let $s$ be the (Euclidean) vertical coordinate of $a_{0}$ and $t$ the one of $a$, with $0<t \leq s \leq 1$. 


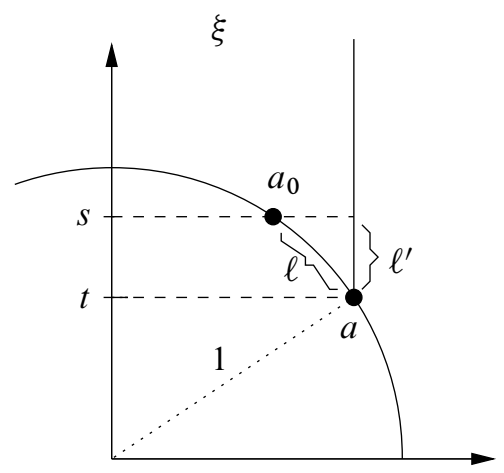

An easy computation in hyperbolic geometry (see also the proof of Lemma 2.1) gives $\ell^{\prime}=\log \frac{s}{t}$ and

$$
\ell=\operatorname{arsinh} \frac{\sqrt{1-t^{2}}}{t}-\operatorname{arsinh} \frac{\sqrt{1-s^{2}}}{s}=\log \frac{s}{t}+\log \frac{1+\sqrt{1-t^{2}}}{1+\sqrt{1-s^{2}}} .
$$

Hence, to prove that $\ell \leq \frac{3}{2} \ell^{\prime}$, we only have to show that

$$
\log \frac{1+\sqrt{1-t^{2}}}{1+\sqrt{1-s^{2}}} \leq \frac{1}{2} \log \frac{s}{t}
$$

which is equivalent to $\sqrt{t}\left(1+\sqrt{1-t^{2}}\right) \leq \sqrt{s}\left(1+\sqrt{1-s^{2}}\right)$. The map $f: x \mapsto$ $\sqrt{x}\left(1+\sqrt{1-x^{2}}\right)$ on $[0,1]$ is increasing from $f(0)=0$ to $f(\sqrt{5} / 3)$, and then decreasing to $f(1)=1$. Let $t^{\prime}=0.25873$. As $f\left(t^{\prime}\right)<1$ and $s \geq t$, to prove that $f(t) \leq f(s)$, it is sufficient to show that $t \leq t^{\prime}$. Let $a^{\prime}$ and $b^{\prime}$ be the two points of the unit circle at (Euclidean) height $t^{\prime}$. As $a$ and $b$ are at the same (Euclidean) height $t$ on the unit circle, to prove that $t \leq t^{\prime}$, we only have to show that $d\left(a^{\prime}, b^{\prime}\right) \leq d(a, b)$. By the definition of $c_{0}(\infty)$, we have

$$
d\left(a^{\prime}, b^{\prime}\right)=2 \operatorname{arsinh} \frac{\sqrt{1-t^{\prime 2}}}{t^{\prime}} \leq c_{0}(\infty) \leq d(a, b) .
$$

Hence the result follows.

Lemma 2.11 For every horoball $H$ in $X$, for every $\xi_{0}$ in $X \cup \partial_{\infty} X$, for all geodesic rays or lines $\gamma$ and $\gamma^{\prime}$ starting from $\xi_{0}$ and entering $H$ in $x \in X$ and $x^{\prime} \in X$ respectively, if the length of $\gamma^{\prime} \cap H$ is at least $c_{0}(\infty)$, then

$$
d\left(x, x^{\prime}\right) \leq \frac{5}{2} d\left(x, \gamma^{\prime}\right) .
$$


Proof Let $p^{\prime}$ be the point of $\gamma^{\prime}$ the closest to $x$. Let $\xi$ be the point at infinity of $H$. Define $y^{\prime}$ by $\left[x^{\prime}, y^{\prime}\right]=\gamma^{\prime} \cap H$ if this intersection is bounded, and $y^{\prime}=\xi$ otherwise. We may assume that $x \neq x^{\prime}$. In particular, $\xi_{0} \notin H \cup\{\xi\}$.

Assume first that $p^{\prime}$ does not belong to $\left[\xi_{0}, x^{\prime}\right.$. As closest point projections do not increase distances and by Lemma 2.9, we have $d\left(x^{\prime}, p^{\prime}\right) \leq d\left(x, x^{\prime}\right) \leq c_{1}^{\prime}(\infty)$. Since

$$
d\left(x^{\prime}, y^{\prime}\right) \geq c_{0}(\infty) \geq 2 c_{1}^{\prime}(\infty),
$$

the point $p^{\prime}$ belongs to $H$, and $d\left(p^{\prime}, y^{\prime}\right) \geq d\left(p^{\prime}, x^{\prime}\right)$. Let $z$ be the point of intersection of $\left.] \xi, x^{\prime}\right]$ with the horosphere centered at $\xi$ passing through $p^{\prime}$, so that in particular $d\left(x, p^{\prime}\right) \geq d\left(x^{\prime}, z\right)$. By Lemma 2.10, we have $d\left(x^{\prime}, z\right) \geq \frac{2}{3} d\left(x^{\prime}, p^{\prime}\right)$. Hence

$$
d\left(x, x^{\prime}\right) \leq d\left(x, p^{\prime}\right)+d\left(p^{\prime}, x^{\prime}\right) \leq d\left(x, p^{\prime}\right)+\frac{3}{2} d\left(x^{\prime}, z\right) \leq \frac{5}{2} d\left(x, p^{\prime}\right) .
$$

Assume now that $p^{\prime}$ belongs to $\left[\xi_{0}, x^{\prime}\left[\right.\right.$. Let $\beta$ be the comparison angle at $x^{\prime}$ between the (nontrivial) geodesic segments or rays $\left[x^{\prime}, y^{\prime}\left[\right.\right.$ and $\left[x^{\prime}, \xi[\right.$. By comparison, $\beta$ is at most the angle $\bar{\beta}$ between $\left[\overline{x^{\prime}}, \overline{y^{\prime}}\left[\right.\right.$ and $\left[\overline{x^{\prime}}, \bar{\xi}\right.$, where $\overline{x^{\prime}}$ and $\overline{y^{\prime}}$ are two points, at distance $d\left(x^{\prime}, y^{\prime}\right)$, on a horosphere in $\mathbb{H}_{\mathbb{R}}^{2}$ centered at $\bar{\xi}$. An easy computation in the upper half space model and the inequality (10) show that

$$
\tan \bar{\beta}=\left(\sinh \frac{1}{2} d\left(\overline{x^{\prime}}, \overline{y^{\prime}}\right)\right)^{-1} \leq(\sinh (2 \log (1+\sqrt{2})))^{-1} \leq \frac{1}{\sqrt{3}} .
$$

As $0 \leq \bar{\beta} \leq \frac{\pi}{2}$, this implies that $\beta \leq \bar{\beta} \leq \frac{\pi}{6}$.

Let $\alpha$ be the comparison angle at $x^{\prime}$ between the (nontrivial) geodesic segments $\left[x^{\prime}, p^{\prime}\right]$ and $\left[x^{\prime}, x\right]$, which is at most $\frac{\pi}{2}$, as $p^{\prime}$ is the closest point to $x$ on $\left.] x^{\prime}, \xi_{0}\right]$. As the geodesic segment $\left[x, x^{\prime}\right]$ lies in $H$, we have $\alpha \geq \pi-\frac{\pi}{2}-\beta \geq \frac{\pi}{3}$. By Lemma 2.9, we have $d\left(x, p^{\prime}\right) \leq d\left(x, x^{\prime}\right) \leq c_{1}^{\prime}(\infty)$. Using the formulae for right-angled hyperbolic triangles in [5] and the comparison triangle in $\mathbb{H}_{\mathbb{R}}^{2}$ to the triangle $\left(x, x^{\prime}, p^{\prime}\right)$ in $X$, we have, by convexity of $t \mapsto \sinh t$,

$d\left(x, x^{\prime}\right) \leq \sinh d\left(x, x^{\prime}\right) \leq \frac{1}{\sin \alpha} \sinh d\left(x, p^{\prime}\right) \leq \frac{2}{\sqrt{3}} \frac{\sinh c_{1}^{\prime}(\infty)}{c_{1}^{\prime}(\infty)} d\left(x, p^{\prime}\right) \leq \frac{5}{2} d\left(x, p^{\prime}\right)$.

This proves the result.

The following Lemma is the analog of Lemma 2.6 for horoballs. It says that when the $\epsilon$-convex subset under consideration is a horoball, we can replace $c_{3}^{\prime}(\epsilon)$ and $h^{\prime}(\epsilon, \eta)$ by

$$
c_{3}^{\prime}(\infty)=\frac{5}{2} \quad \text { and } \quad h^{\prime}(\infty, \eta)=3 \eta+c_{0}(\infty)+c_{1}^{\prime}(\infty) \approx 3 \eta+5.8188
$$


and that the first of the two possible conclusions of Lemma 2.6 always holds. Note that $c_{3}^{\prime}(\infty)$ is not the limit as $\epsilon$ goes to $+\infty$ of $c_{3}^{\prime}(\epsilon)$, and that $h^{\prime}(\epsilon, \eta)$ diverges as $\epsilon \rightarrow \infty$. However, in both cases, this mnemonic notation will be useful in Section 4, where it will be used in a similar way whether $\epsilon$ is finite or not.

Lemma 2.12 For every horoball $H$ in $X$, for every $\xi_{0}$ in $\left(X \cup \partial_{\infty} X\right)-(H \cup H[\infty])$, for all geodesic rays or lines $\gamma, \gamma^{\prime}$ starting from $\xi_{0}$, if $\gamma$ enters $H$ at a point $x \in X$ and exits $H$ at a point $y \in X$, and if $d(x, y) \geq h^{\prime}\left(\infty, d\left(y, \gamma^{\prime}\right)\right)$, then $\gamma^{\prime}$ meets $H$, exiting it at a point $y^{\prime} \in X$ such that

$$
d\left(y, y^{\prime}\right) \leq \frac{5}{2} d\left(y, \gamma^{\prime}\right) .
$$

Proof Let $\xi$ be the point at infinity of $H$, let $p$ be the closest point on $[x, y]$ to $\xi$, and let $p_{x}$ and $p_{y}$ be the points of intersection of the horosphere $\partial H_{p}$ centered at $\xi$ passing through $p$ with the geodesic rays $[x, \xi$ [ and $[y, \xi$ [ respectively.

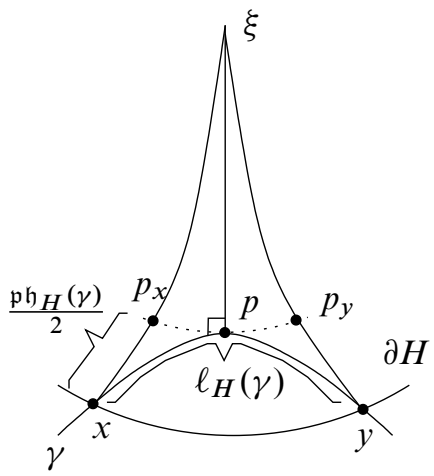

By comparison, we have $d\left(p_{x}, p_{y}\right) \leq 2 \log (1+\sqrt{2})=c_{1}^{\prime}(\infty)$. Thus, the triangle inequality, along with the fact that $p_{y}$ is the closest point to $y$ on $\partial H_{p}$ and the assumption on $d(x, y)$, gives

$2 \min \{d(y, p), d(x, p)\} \geq d\left(y, p_{y}\right)+d\left(x, p_{x}\right) \geq d(x, y)-2 \log (1+\sqrt{2}) \geq c_{0}(\infty) \geq 3$.

In particular, as $d(x, y) \geq c_{0}(\infty)$, Lemma 2.10 implies that $p$ belongs to $H[1]$. By Lemma 2.1 and the assumption on $d(x, y)$, we have

$$
d\left(p, \gamma^{\prime}\right) \leq \frac{1}{2} e^{-d(y, p)+d\left(y, \gamma^{\prime}\right)} \leq \frac{1}{2} e^{-(1 / 2) d(x, y)+\log (1+\sqrt{2})+d\left(y, \gamma^{\prime}\right)} \leq \frac{1}{2} .
$$

This implies that $\gamma^{\prime}$ meets $H$, because $\mathcal{N}_{1 / 2}(H[1])=H\left[\frac{1}{2}\right]$ is contained in $H$. Let $x^{\prime}$ and $y^{\prime}$ be the entering point in $H$ and the exiting point out of $H$ of $\gamma^{\prime}$, respectively. Let $p^{\prime}$ be the point on $\gamma^{\prime}$ the closest to $y$. 
Case 1 Assume that $p^{\prime} \notin\left[y^{\prime}, \xi_{0}\right]$. Note that

$$
d(x, y)-\frac{3}{2} d\left(y, p^{\prime}\right) \geq \frac{3}{2} d\left(y, p^{\prime}\right) \geq 0,
$$

as $d(x, y) \geq 3 d\left(y, \gamma^{\prime}\right)$, by the definition of $h^{\prime}(\infty, \eta)$. Hence, there is a point $y_{0}$ in $[x, y]$ at distance $\frac{3}{2} d\left(y, p^{\prime}\right)$ of $y$ which satisfies $d\left(x, y_{0}\right) \geq \frac{3}{2} d\left(y, p^{\prime}\right)$. By Lemma 2.10, we have $y_{0} \in H\left[d\left(y, p^{\prime}\right)\right]$. Let $q^{\prime}$ be the point of $\gamma^{\prime}$ the closest to $y_{0}$. By convexity, we have $d\left(y_{0}, q^{\prime}\right) \leq d\left(y, p^{\prime}\right)$. Hence $q^{\prime}$ belongs to $H$. By the intermediate value theorem, the point $y^{\prime}$ belongs to $\left[q^{\prime}, p^{\prime}\right]$. As closest point maps do not increase the distances, we have $d\left(p^{\prime}, q^{\prime}\right) \leq d\left(y, y_{0}\right)=\frac{3}{2} d\left(y, p^{\prime}\right)$. Therefore,

$$
d\left(y, y^{\prime}\right) \leq d\left(y, p^{\prime}\right)+d\left(p^{\prime}, y^{\prime}\right) \leq d\left(y, p^{\prime}\right)+d\left(p^{\prime}, q^{\prime}\right) \leq \frac{5}{2} d\left(y, p^{\prime}\right),
$$

which proves the result.

Case 2 Assume that $\left.\left.p^{\prime} \in\right] y^{\prime}, \xi_{0}\right]$. By the same argument as in Case 2 of the proof of Lemma 2.6, we have $p^{\prime} \notin\left[x^{\prime}, \xi_{0}\right]$. If $d\left(p^{\prime}, y^{\prime}\right) \leq \frac{3}{2} d\left(y, p^{\prime}\right)$, then $d\left(y, y^{\prime}\right) \leq \frac{5}{2} d\left(y, p^{\prime}\right)$, and the result holds. So assume by contradiction that $d\left(p^{\prime}, y^{\prime}\right)>\frac{3}{2} d\left(y, p^{\prime}\right)$. By the continuity of the closest point maps, there exists a point $y_{0}$ in $\gamma$ that does not belong to $H$, whose closest point $q^{\prime}$ on $\gamma^{\prime}$ belongs to $\left.] p^{\prime}, y^{\prime}\right]$ and satisfies $d\left(q^{\prime}, y^{\prime}\right) \geq$ $\frac{3}{2} d\left(y_{0}, q^{\prime}\right)$ and $d\left(y_{0}, q^{\prime}\right) \leq d\left(y, p^{\prime}\right)+\frac{1}{2} c_{0}(\infty)$. Lemma 2.9 implies that $d\left(x, x^{\prime}\right) \leq$ $c_{1}^{\prime}(\infty)$. Thus, by the assumption on $d(x, y)$,

$$
\begin{aligned}
d\left(x^{\prime}, y^{\prime}\right) & \geq d\left(x^{\prime}, q^{\prime}\right) \geq d\left(p^{\prime}, x^{\prime}\right) \geq d(x, y)-d\left(x, x^{\prime}\right)-d\left(y, p^{\prime}\right) \\
& \geq 3 d\left(y, p^{\prime}\right)+c_{0}(\infty)+c_{1}^{\prime}(\infty)-c_{1}^{\prime}(\infty)-d\left(y, p^{\prime}\right) \\
& \geq 2 d\left(y, p^{\prime}\right)+c_{0}(\infty) \geq \max \left\{2 d\left(y_{0}, q^{\prime}\right), c_{0}(\infty)\right\} .
\end{aligned}
$$

In particular, $d\left(y_{0}, q^{\prime}\right) \leq \frac{1}{2} d\left(x^{\prime}, q^{\prime}\right) \leq \frac{2}{3} d\left(q^{\prime}, x^{\prime}\right)$ and we already had $d\left(y_{0}, q^{\prime}\right) \leq$ $\frac{2}{3} d\left(q^{\prime}, y^{\prime}\right)$. Hence, by Lemma 2.10, we have $q^{\prime} \in H\left[d\left(y_{0}, q^{\prime}\right)\right]$. This implies that $y_{0}$ belongs to $H$, a contradiction.

\section{Properties of penetration in $\epsilon$-convex sets}

\subsection{Penetration maps}

Let $X$ be a proper geodesic CAT(-1) space, and $\xi_{0} \in X \cup \partial_{\infty} X$. We are interested in controlling the penetration of geodesic rays or lines starting from $\xi_{0}$ in $\epsilon$-convex subsets of $X$. One way to measure this penetration is the intersection length. If $C$ is a closed convex subset in $X$ such that $\xi_{0} \notin C \cup \partial_{\infty} C$, we define a map $\ell_{C}: T_{\xi_{0}}^{1} X \rightarrow[0,+\infty]$, 
called the penetration length map, which associates to every $\gamma$ in $T_{\xi_{0}}^{1} X$ the length of the intersection $\gamma \cap C$ (which is connected by convexity).

When we study specific geometric situations, such as collections of horoballs and $\epsilon$-neighbourhoods of geodesics, there are further natural ways of measuring the penetration. These will be used in many applications in Section 5 and in [49]. If $C$ is an $\epsilon$-convex subset of $X$ such that $\xi_{0} \notin C \cup \partial_{\infty} C$, we will require our penetration maps $f: T_{\xi_{0}}^{1} X \rightarrow[0,+\infty]$ in $C$ to have one or two of the following properties, the first one depending on a constant $\kappa \geq 0$. The sup-norm of a real valued function $f$ on $T_{\xi_{0}}^{1} X$ is denoted by $\|f\|_{\infty}$.

(i) (Penetration property) For any $\gamma$ in $T_{\xi_{0}}^{1} X, f(\gamma)=+\infty$ if and only if $\ell_{C}(\gamma)=$ $+\infty$, and the restrictions of $f$ and $\ell_{C}$ to the set where both functions are finite satisfy $\left\|f-\ell_{C}\right\|_{\infty} \leq \kappa$.

(ii) (Lipschitz property) For every $\gamma, \gamma^{\prime}$ in $T_{\xi_{0}}^{1} X$ which intersect $C$, if $\gamma \cap C=[a, b]$ and $\gamma^{\prime} \cap C=\left[a^{\prime}, b^{\prime}\right]$ with $a, b, a^{\prime}, b^{\prime}$ in $X$, then

$$
\left|f(\gamma)-f\left(\gamma^{\prime}\right)\right| \leq 2 \max \left\{d\left(a, a^{\prime}\right), d\left(b, b^{\prime}\right)\right\} .
$$

If $C$ is an $\epsilon$-convex subset of $X$ such that $\xi_{0} \notin C \cup \partial_{\infty} C$, and $f: T_{\xi_{0}}^{1} X \rightarrow[0,+\infty[$ is a map which satisfies (i) for some $\kappa \geq 0$, we say that $f$ is a $\kappa$-penetration map in (the $\epsilon$-convex set) $C$. We also say that $(C, f)$ is an $(\epsilon, \kappa)$-penetration pair. In the condition (ii), we could have replaced 2 by some $\lambda \geq 2$, but if $f$ also satisfies the property (i), then only $\lambda=2$ is really relevant in the large scale.

Note that if $(C, f)$ is an $\left(\epsilon^{\prime}, \kappa^{\prime}\right)$-penetration pair, if $\epsilon^{\prime} \geq \epsilon$ and $\kappa^{\prime} \leq \kappa$, then $(C, f)$ is an $(\epsilon, \kappa)$-penetration pair. If $C$ is $\infty$-convex and $(C, f)$ is an $(\epsilon, \kappa)$-penetration pair in every $\epsilon>0$ then $f$ will be called a $\kappa$-penetration map in (the $\infty$-convex set) $C$.

Penetration maps in general $\epsilon$-convex subsets If $C$ is a closed convex subset of $X$, the map $\ell_{C}$ is in general not continuous on $T_{\xi_{0}}^{1} X$, as can be seen by taking $C$ to be a geodesic segment of positive length. The following result shows that the situation is nicer for $\epsilon$-convex subsets. Note that the statement of Lemma 3.1 is not true in $\mathbb{R}^{n}$ (which is not a CAT(-1) space).

Lemma 3.1 Let $\epsilon>0$ and let $C$ be an $\epsilon$-convex subset of $X$ such that $\xi_{0} \notin C \cup \partial_{\infty} C$. The map $\ell_{C}: T_{\xi_{0}}^{1} X \rightarrow[0,+\infty]$ is a continuous 0 -penetration map in $C$ satisfying the Lipschitz property (ii).

Proof The Lipschitz property (ii) of the penetration length map $\ell_{C}$ follows from the triangle inequality. It remains to show the continuity of the map. 
Choose a convex subset $C^{\prime}$ such that $C=\mathcal{N}_{\epsilon}\left(C^{\prime}\right)$, and note that by the definition of the topology of $X \cup \partial_{\infty} X$, the subsets $C$ and $C^{\prime}$ have the same points at infinity. Let $\gamma_{0} \in T_{\xi_{0}}^{1} X$, and let us prove that $\ell_{C}$ is continuous at $\gamma_{0}$.

Assume first that $\gamma_{0}(+\infty)$ is a point at infinity of $C$. Then there exists a geodesic ray contained in $C^{\prime}$ ending at this point at infinity. As geodesic rays converging to the same point at infinity become exponentially close, this implies that $\ell_{C}\left(\gamma_{0}\right)=\infty$. Let $A>0$. As $\gamma_{0} \cap C$ is the closure of $\gamma_{0} \cap \stackrel{\circ}{C}$, let $[x, y]$ be a geodesic segment of length $A+2$ contained in $\gamma_{0} \cap \stackrel{\circ}{C}$. Let $\left.\left.\eta \in\right] 0,1\right]$ be such that the balls $B$ and $B^{\prime}$ of radius $\eta$ and of center $x$ and $y$ respectively are contained in $\stackrel{\circ}{C}$. If $\gamma \in T_{\xi_{0}}^{1} X$ is close enough to $\gamma_{0}$, then $\gamma$ meets $B$ and $B^{\prime}$, and by convexity, $\ell_{C}(\gamma) \geq A$, which proves the result.

Assume now that $\gamma_{0}(+\infty)$ is not a point at infinity of $C$, but that $\gamma_{0}$ does meet $C$. Then $\gamma_{0} \cap C$ is a nonempty compact segment $[a, b]$. For every $\eta>0$, let $a_{+}, b_{+}$be points in $\gamma_{0}-[a, b]$, at distance at most $\eta / 4$ from $a, b$ respectively, and, if $d(a, b)>0$, let $a_{-}, b_{-}$be points in ] $a, b$ [ at distance at most $\eta / 4$ from $a, b$ respectively. As $C$ is closed and $\gamma_{0} \cap C$ is the closure of $\gamma_{0} \cap \stackrel{\circ}{C}$ if $a \neq b$, there exists $\left.\left.\eta^{\prime} \in\right] 0, \eta / 4\right]$ such that the balls $B\left(a_{+}\right), B\left(b_{+}\right)$of radius $\eta^{\prime}$ and centers $a_{+}, b_{+}$respectively are contained in $X-C$ and, if $d(a, b)>0$, the balls $B\left(a_{-}\right), B\left(b_{-}\right)$of radius $\eta^{\prime}$ and centers $a_{-}, b_{-}$ respectively are contained in the interior of $C$. If $\gamma \in T_{\xi_{0}}^{1} X$ is close enough to $\gamma_{0}$, then $\gamma$ meets $B\left(a_{+}\right), B\left(b_{+}\right)$(and hence $B\left(a_{-}\right), B\left(b_{-}\right)$by convexity, if $d(a, b)>0$ ). It is easy to see then that $\left|\ell_{C}(\gamma)-\ell_{C}\left(\gamma_{0}\right)\right| \leq \eta$.

Assume now that $\gamma_{0}$ does not meet $C$. Let $U, V$ be neighbourhoods of the endpoints of $\gamma_{0}$ in $X \cup \partial_{\infty} X$ that are disjoint from $C \cup \partial_{\infty} C$. Let $\eta>0$ be such that the $\eta$-neighbourhood of $\gamma_{0}$ is disjoint from $C$, which exists, as $\inf _{x \in \gamma_{0}} d(x, C)>0$. If $\gamma \in T_{\xi_{0}}^{1} X$ is close enough to $\gamma_{0}$, then (the image of) $\gamma$ lies in $U \cup V \cup \mathcal{N}_{\eta} \gamma_{0}$, hence does not meet $C$. Thus, $\ell_{C}(\gamma)=\ell_{C}\left(\gamma_{0}\right)=0$.

In particular, if $H$ is a horoball such that $\xi_{0} \notin H \cup \partial_{\infty} H$, then $\ell_{H}$ is a continuous 0 -penetration map for $H$ satisfying the Lipschitz property (ii).

Let $C$ be a convex subset of $X$ such that $\xi_{0} \notin C \cup \partial_{\infty} C$. For every $\gamma$ in $T_{\xi_{0}}^{1} X$, let $\gamma_{-}=\xi_{0}$ and $\gamma_{+}=\gamma(+\infty)$, and let $q_{\gamma_{ \pm}}$be the closest point on $C$ to $\gamma_{ \pm}$. Define the boundary-projection penetration map $\mathfrak{b p}_{C}: T_{\xi_{0}}^{1} X \rightarrow[0,+\infty]$ by

$$
\mathfrak{b p}_{C}(\gamma)=d\left(q_{\gamma_{-}}, q_{\gamma_{+}}\right) \text {, }
$$

with the obvious convention that $\mathfrak{b p}_{C}(\gamma)=+\infty$ if $q_{\gamma_{+}}$is at infinity.

Lemma 3.2 Let $C$ be an $\epsilon$-convex subset of $X$ such that $\xi_{0} \notin C \cup \partial_{\infty} C$. The map $\mathfrak{b p}_{C}$ is a continuous $2 c_{1}^{\prime}(\epsilon)$-penetration map in $C$. 
Proof The continuity of $\mathfrak{b p}_{C}$ follows from the continuity of the projection maps and the endpoint maps. Let us prove that $\mathfrak{b p}_{C}$ has the penetration property (i) with $\kappa=$ $2 c_{1}^{\prime}(\epsilon)$. Let $\gamma \in T_{\xi_{0}}^{1} X$. If $\gamma_{+}$is a point at infinity of $C$, then $\mathfrak{b p}_{C}(\gamma)=\ell_{C}(\gamma)=+\infty$, and the claim is true for these geodesics. Otherwise, if $\gamma$ meets $C$, then $\gamma$ enters $C$ at $x$ and exits $C$ at $y$, with $x, y$ in $X$. By Lemma 2.3, we hence have

$$
\left|d(x, y)-d\left(q_{\gamma_{-}}, q_{\gamma_{+}}\right)\right| \leq d\left(x, q_{\gamma_{-}}\right)+d\left(q_{\gamma_{+}}, y\right) \leq 2 c_{1}^{\prime}(\epsilon),
$$

and the result follows.

If $\gamma$ does not meet $C$, let $[p, q]$ be the shortest connecting segment between a point $p$ in $\gamma$ and a point $q$ in $C$. By angle comparison, the geodesic segment or ray between $q$ and $\gamma_{ \pm}$meets $C$ exactly in $q$. Hence, by Lemma 2.3,

$$
d\left(q_{\gamma_{-}}, q_{\gamma_{+}}\right) \leq d\left(q_{\gamma_{-}}, q\right)+d\left(q, q_{\gamma_{+}}\right) \leq 2 c_{1}^{\prime}(\epsilon) .
$$

As $\ell_{C}(\gamma)=0$, the result follows.

Penetration maps in horoballs If $H$ is a horoball in $X$, with $\xi$ its point at infinity, such that $\xi_{0} \notin H \cup\{\xi\}$, and if $x_{0}$ is any point in the boundary of $H$ in $X$, define a 1 -Lipschitz map $\beta_{H}: X \rightarrow[0,+\infty[$, called the height map of $H$, by

$$
\beta_{H}: x \mapsto \max \left\{\beta_{\xi}\left(x_{0}, x\right), 0\right\},
$$

whose values are positive in the interior of $H$, and 0 outside $H$. By convention, define $\beta_{H}(\xi)=+\infty$. Note that $\beta_{H}$ is independent of the choice of the point $x_{0}$. For every $\gamma$ in $T_{\xi_{0}}^{1} X$, let $p_{\gamma}$ be the closest point to $\gamma(+\infty)$ on the geodesic line between $\xi_{0}$ and $\xi$, with $p_{\gamma}=\xi$ if $\gamma(+\infty)=\xi$.

We will study two penetration maps associated with the height map. We will call the map $\mathfrak{p h}_{H}: T_{\xi_{0}}^{1} X \rightarrow[0,+\infty]$ defined by

$$
\mathfrak{p h}_{H}(\gamma)=2 \sup _{t \in \mathbb{R}} \beta_{H}(\gamma(t))
$$

the penetration height map inside $H$. The map $\mathfrak{i p p}_{H}: T_{\xi_{0}}^{1} X \rightarrow[0,+\infty]$ defined by

$$
\mathfrak{i p p}_{H}(\gamma)=2 \beta_{H}\left(p_{\gamma}\right)
$$

will be called the inner-projection penetration map inside $H$. Note that for every $t \geq 0$ and $\gamma \in T_{\xi_{0}}^{1} X$, we have $\mathfrak{p h}_{H[t]}(\gamma)=\max \left\{0, \mathfrak{p h}_{H}(\gamma)-2 t\right\}$ and $\mathfrak{i p p}_{H[t]}(\gamma)=$ $\max \left\{0, \mathfrak{i p p}_{H}(\gamma)-2 t\right\}$. 
Lemma 3.3 Let $H$ be a horoball in $X$, such that $\xi_{0} \notin H \cup \partial_{\infty} H$. The maps $\mathfrak{p h}_{H}, \mathfrak{i p p}_{H}: T_{\xi_{0}}^{1} X \rightarrow[0,+\infty]$ are continuous $2 \log (1+\sqrt{2})$-penetration maps for $H$, and $\mathfrak{p h}_{H}$ has the Lipschitz property (ii). Furthermore,

$$
\left\|\mathfrak{p h} \mathfrak{h}_{H}-\mathfrak{i p p}_{H}\right\|_{\infty} \leq 2 \log (1+\sqrt{2}) .
$$

Remark In $\mathbb{H}_{\mathbb{R}}^{n}$, the equality $\mathfrak{i p p}_{H}(\gamma)=\mathfrak{p h}_{H}(\gamma)+\log 2$ holds for any horoball $H$ with $\xi_{0} \notin H \cup H[\infty]$ and $\gamma \in T_{\xi_{0}}^{1} X$ meeting $H$. Thus, in $\mathbb{H}_{\mathbb{R}}^{n}$ the map ipp $\mathfrak{p p}_{H}$ satisfies the Lipschitz property (ii). We do not know whether $\left(H, \mathfrak{i p p}_{H}\right)$ satisfies the Lipschitz property (ii) in general.

Proof Let us prove that $\left(H, \mathfrak{p h}_{H}\right)$ satisfies the Lipschitz property (ii). Let $\gamma, \gamma^{\prime}$ be elements in $T_{\xi_{0}}^{1} X$ such that $\gamma \cap H=[a, b]$ and $\gamma^{\prime} \cap H=\left[a^{\prime}, b^{\prime}\right]$. Then, for every $x$ in $[a, b]$, if $x^{\prime}$ is the point on $\left[a^{\prime}, b^{\prime}\right]$ the closest to $x$, we have, with $\xi$ the point at infinity of $H$,

$$
\left|\beta_{\xi}(a, x)-\beta_{\xi}\left(a, x^{\prime}\right)\right|=\left|\beta_{\xi}\left(x, x^{\prime}\right)\right| \leq d\left(x, x^{\prime}\right) \leq \max \left\{d\left(a, a^{\prime}\right), d\left(b, b^{\prime}\right)\right\}
$$

by convexity. Taking $x$ the highest point in $[a, b]$, we get

$$
\begin{aligned}
\mathfrak{p h}_{H}(\gamma)=2 \beta_{\xi}(a, x) & \leq 2 \max \left\{d\left(a, a^{\prime}\right), d\left(b, b^{\prime}\right)\right\}+2 \beta_{\xi}\left(a, x^{\prime}\right) \\
& \leq 2 \max \left\{d\left(a, a^{\prime}\right), d\left(b, b^{\prime}\right)\right\}+\mathfrak{p h}_{H}\left(\gamma^{\prime}\right) .
\end{aligned}
$$

Using a symmetry argument, the result follows.

Let us prove that $\left(H, \mathfrak{p h}_{H}\right)$ satisfies the penetration property (i) with $\kappa=c_{1}^{\prime}(\infty)=$ $2 \log (1+\sqrt{2})$. Let $\gamma \in T_{\xi_{0}}^{1} X$. Note that $\gamma$ enters the interior of $H$ if and only if $\ell_{H}(\gamma)>0$, and if and only if $\mathfrak{p h}_{H}(\gamma)>0$. Hence we may assume that $\gamma$ meets $H$ in a segment $[x, y]$. By the first paragraph of the proof of Lemma 2.12, we have

$$
\mathfrak{p h} \mathfrak{h}_{H}(\gamma) \leq \ell_{H}(\gamma) \leq \mathfrak{p h} \mathfrak{h}_{H}(\gamma)+2 \log (1+\sqrt{2}) \text {. }
$$

Let us now prove that $\left(H, \mathfrak{i p p}_{H}\right)$ satisfies the penetration property (i) with $\kappa=$ $2 \log (1+\sqrt{2})$. Let $\gamma \in T_{\xi_{0}}^{1} X$. If $p_{\gamma}=\xi$, then $\mathfrak{i p p}_{H}(\gamma)=\ell_{H}(\gamma)=+\infty$, and the result holds, hence we may assume that $p_{\gamma}$ belongs to $X$. If $p_{\gamma}$ does not belong to $H$, as the closest point projection of $\gamma$ on the geodesic line $] \gamma_{-}, \xi$ [is $] \gamma_{-}, p_{\gamma}$ [, then $\gamma$ does not enter $H$, and hence $\mathfrak{i p p}_{H}(\gamma)=\ell_{H}(\gamma)=0$, and the result is proven.

Assume that $p_{\gamma}$ belongs to $H$, and note that by comparison and an easy hyperbolic estimate, we have $d\left(p_{\gamma}, \gamma\right) \leq \log (1+\sqrt{2})$. 


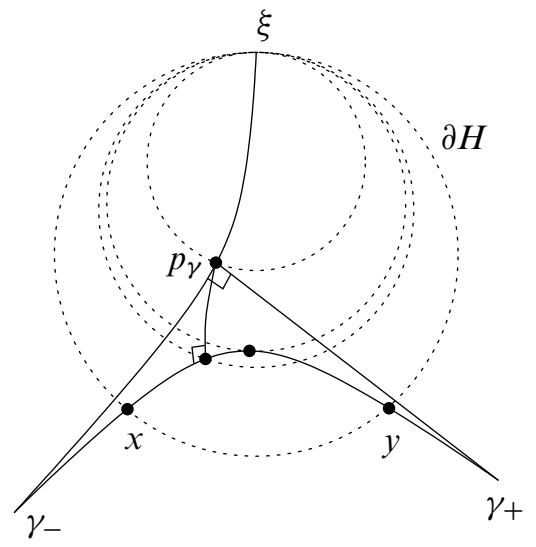

In particular, if $\gamma$ does not enter $H$, then $0 \leq \beta_{H}\left(p_{\gamma}\right) \leq d\left(p_{\gamma}, \gamma\right) \leq \log (1+\sqrt{2})$, and $\left|\mathfrak{i p p}_{H}(\gamma)-\ell_{H}(\gamma)\right| \leq 2 \log (1+\sqrt{2})$, hence the result holds. Therefore we may assume that $\gamma$ enters $H$ at the point $x$ and exits $H$ at the point $y$. We then have $\mathfrak{p h}_{H}(\gamma) \leq \mathfrak{i p p}_{H}(\gamma) \leq \mathfrak{p h}_{H}(\gamma)+2 d\left(p_{\gamma}, \gamma\right)$. Hence,

$$
\ell_{H}(\gamma)-2 \log (1+\sqrt{2}) \leq \mathfrak{i p p}_{H}(\gamma) \leq \ell_{H}(\gamma)+2 \log (1+\sqrt{2}),
$$

and the result is proven. The continuity of $\mathfrak{i p p}_{H}$ follows from the continuity of the endpoint maps, of the closest point projection maps and of $\beta_{H}: X \cup\{\xi\} \rightarrow[0,+\infty]$. To prove the continuity of $\mathfrak{p h}_{H}$ at a point $\gamma_{0}$ of $T_{\xi_{0}}^{1} X$, note that if $\gamma_{0}(+\infty)=\xi$, then $\mathfrak{p h}_{H}\left(\gamma_{0}\right)=+\infty$, and the continuity follows from the penetration property (i) of $\left(H, \mathfrak{p h}_{H}\right)$ and the continuity of $\ell_{H}$. Otherwise, $\gamma_{0} \cap H$ is a compact segment. If it is nonempty, then if $\gamma$ is close enough to $\gamma^{\prime}$, the argument in the proof of Lemma 3.1 shows that the Hausdorff distance between $\gamma \cap H$ and $\gamma_{0} \cap H$ is as small as wanted. The result follows then since $\beta_{H}$ is 1-Lipschitz (and vanishes outside $H$ ). If $\gamma_{0}$ does not meet $H$, then if $\gamma$ is close enough to $\gamma^{\prime}$, the argument in the proof of Lemma 3.1 shows that $\gamma$ also avoids $H$, hence $\mathfrak{p h}_{H}(\gamma)=\ell_{H}(\gamma)=0$.

Penetration maps in balls If $B$ is a ball of center $x_{0}$ and radius $r_{0}$ in $X$ with $\xi_{0} \notin B$, define a 1-Lipschitz map $\beta_{B}: X \rightarrow[0,+\infty[$, called the height map, by

$$
\beta_{B}: x \mapsto \max \left\{r_{0}-d\left(x_{0}, x\right), 0\right\},
$$

whose values are positive in the interior of $B$, and 0 outside $B$. For every $\gamma$ in $T_{\xi_{0}}^{1} X$, let $p_{\gamma}$ be the closest point to $\gamma(+\infty)$ on the geodesic segment or ray between $\xi_{0}$ and $x_{0}$.

The map $\mathfrak{p h}_{B}: T_{\xi_{0}}^{1} X \rightarrow\left[0,2 r_{0}\right]$ defined by

$$
\mathfrak{p h}_{B}(\gamma)=2 \sup _{t \in \mathbb{R}} \beta_{B}(\gamma(t))
$$


will be called the penetration height map inside $B$. The map $\mathfrak{i p p}_{B}: T_{\xi_{0}}^{1} X \rightarrow\left[0,2 r_{0}\right]$ defined by

$$
\mathfrak{i p p}_{B}(\gamma)=2 \beta_{B}\left(p_{\gamma}\right)
$$

will be called the inner-projection penetration map inside $B$.

We claim that the maps $\mathfrak{p h}_{B}, \mathfrak{i p p}_{B}$ are continuous $2 \log (1+\sqrt{2})$-penetration maps, and that $\mathfrak{p h}_{B}$ has the Lipschitz property (ii); furthermore,

$$
\left\|\mathfrak{p h} \mathfrak{h}_{B}-\mathfrak{i p p}\right\|_{\infty} \leq 2 \log (1+\sqrt{2}) .
$$

This is proved as in Lemma 3.3, except that in the proof of the penetration property (i) of $\mathfrak{i p p}_{B}$, the discussion is on whether $p_{\gamma}$ is equal to $x_{0}$ or not, and if $p_{\gamma}=x_{0}$, then, by comparison, $d\left(\gamma, x_{0}\right) \leq \log (1+\sqrt{2})$, and the claim follows in this case as $\mathfrak{i p p}_{B}(\gamma)=2 r_{0}$.

If a sequence of balls $\left(B_{i}\right)_{i \in \mathbb{N}}$ converges to a horoball $H$ (for the Hausdorff distance on compact subsets of $X$ ), then the maps $\mathfrak{p h}_{B_{i}}, \mathfrak{i p p}_{B_{i}}$ converge, uniformly on compact subsets of $T_{\xi_{0}}^{1} X$, to $\mathfrak{p h}_{H}, \mathfrak{i p p}_{H}$ respectively.

Penetration maps in tubular neighbourhoods of totally geodesic subspaces We define two functions on $T_{\xi_{0}}^{1} X$ which describe the closeness of a geodesic ray or line to a totally geodesic subspace $L$. If $\xi_{0}$ is in the boundary at infinity of $X$, then these functions are defined without reference to an $\epsilon$-neighbourhood of $L$. However, we show that they are penetration maps in the $\epsilon$-neighbourhood of $L$, with explicit constants which depend only on $\epsilon$.

Let $\epsilon>0$, and let $L$ be a complete totally geodesic subspace of $X$, with set of points at infinity $\partial_{\infty} L$, such that $\xi_{0} \notin \mathcal{N}_{\epsilon} L \cup \partial_{\infty} L$. For every $\gamma$ in $T_{\xi_{0}}^{1} X$, let $\gamma_{-}=\xi_{0}$ and $\gamma_{+}=\gamma(+\infty)$, and let $p_{\gamma_{ \pm}}$be the point on $L$ the closest to $\gamma_{ \pm}$.

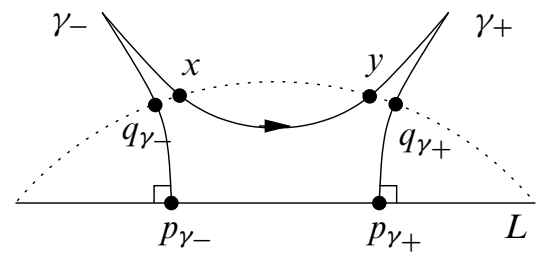

We define the fellow-traveller penetration map $\mathfrak{f t p}_{L}: T_{\xi_{0}}^{1} X \rightarrow[0,+\infty]$ by

$$
\mathfrak{f t p}_{L}(\gamma)=d\left(p_{\gamma_{-}}, p_{\gamma_{+}}\right),
$$

with the convention that this distance is $+\infty$ if $p_{\gamma_{+}}$is in $\partial_{\infty} L$. 
Lemma 3.4 Let $\epsilon>0$, and let $L$ be a complete totally geodesic subspace of $X$ such that $\xi_{0} \notin \mathcal{N}_{\epsilon} L \cup \partial_{\infty} L$. The map $\mathfrak{f t p}_{L}$ is a continuous $\left(2 c_{1}^{\prime}(\epsilon)+2 \epsilon\right)$-penetration map in $\mathcal{N}_{\epsilon} L$ and $\left\|\mathfrak{f} \mathfrak{p}_{L}-\mathfrak{b p}_{\mathcal{N}_{\epsilon} L}\right\|_{\infty} \leq 2 \epsilon$.

Proof The continuity of $\mathfrak{f t p}_{L}$ follows from the continuity of the projection maps and of the endpoint maps. Note that, for every $\gamma$ in $T_{\xi_{0}}^{1} X$, the geodesic segment or ray from $p_{\gamma_{ \pm}}$to $\gamma_{ \pm}$exits $\mathcal{N}_{\epsilon} L$ at the closest point $q_{\gamma_{ \pm}}$on $\mathcal{N}_{\epsilon} L$ to $\gamma_{ \pm}$. Hence, by the triangle inequality, and as closest point maps do not increase distances, we have

$$
0 \leq \mathfrak{b p}_{\mathcal{N}_{\epsilon} L}(\gamma)-\mathfrak{f t p}_{L}(\gamma) \leq 2 \epsilon
$$

Therefore the fact that $\mathfrak{f t p}_{L}(\gamma)$ satisfies the penetration property (i) with $\kappa=2 c_{1}^{\prime}(\epsilon)+2 \epsilon$ follows from Lemma 3.2.

If $L$ is one-dimensional and $\xi_{0} \in \partial_{\infty} X-\partial_{\infty} L$, a natural penetration map is defined using the crossratios of the endpoints of $L$ and $\gamma$. Let $\partial_{4} X$ be the set of quadruples $(a, b, c, d)$ in $\left(\partial_{\infty} X\right)^{4}$ such that $a \neq b$ and $c \neq d$. The crossratio $[a, b, c, d] \in[-\infty,+\infty]$ of a quadruple $(a, b, c, d)$ in $\partial_{4} X$ is defined as follows (see for instance Otal [45], Bourdon [9] and Paulin [51]). If $a_{t}, b_{t}, c_{t}, d_{t}$ are any geodesic rays converging to $a, b, c, d$ respectively, then

$$
[a, b, c, d]=\frac{1}{2} \lim _{t \rightarrow+\infty} d\left(a_{t}, c_{t}\right)-d\left(c_{t}, b_{t}\right)+d\left(b_{t}, d_{t}\right)-d\left(d_{t}, a_{t}\right) .
$$

Note that the order conventions differ in the references, we are using the ones of [9; 29]), and that our crossratio is the logarithm of the crossratio used in [9]. As suggested by the referee, $[a, b, c, d]$ should rather be called "crossdifference", but we will stick to the "crossratio" terminology, which is more widely known, because of formula (12), and because taking a log from the very beginning makes our subsequent formulas shorter.

Let us give other formulae for the crossratio. The visual distance of two points $a$ and $b$ in $\partial_{\infty} X$ with respect to a given point $x_{0}$ in $X$ is

$$
d_{x_{0}}(a, b)=\lim _{t \rightarrow \infty} e^{-\frac{1}{2}\left(d\left(x_{0}, a_{t}\right)+d\left(x_{0}, b_{t}\right)-d\left(a_{t}, b_{t}\right)\right)} .
$$

If $\xi \in \partial_{\infty} X$, if $H$ is a horosphere centered at $\xi$, and $a, b$ are points in $\partial_{\infty} X-\{\xi\}$, and $t \mapsto x_{t}$ is a geodesic ray with $x_{0} \in H$ which converges to $\xi$, the Hamenstädt distance (defined in $\left[26 ; 29\right.$, Appendix]) of $a$ and $b$ in $\partial_{\infty} X-\{\xi\}$ normalized with respect to $H$ is

$$
d_{H}(a, b)=\lim _{t \rightarrow \infty} e^{t} d_{x_{t}}(a, b)
$$


Note that if $H^{\prime}$ is another horosphere centered at $\xi$, then there exists a constant $c>0$ such that $d_{H^{\prime}}=c d_{H}$. In particular, for every $\xi^{\prime} \in \partial_{\infty} X-\{\xi\}$ and $r>0$, the sphere of center $\xi^{\prime}$ and radius $r$ for $d_{H}$ coincides with the sphere of center $\xi^{\prime}$ and radius $\mathrm{cr}$ for $d_{H^{\prime}}$.

It is easy to see that for any $x_{0} \in X$ and any horoball $H$, we have for every $(a, b, c, d) \in$ $\partial_{4} X$

$$
[a, b, c, d]=\log \frac{d_{x_{0}}(a, c)}{d_{x_{0}}(c, b)} \frac{d_{x_{0}}(b, d)}{d_{x_{0}}(d, a)}=\log \frac{d_{H}(a, c)}{d_{H}(c, b)} \frac{d_{H}(b, d)}{d_{H}(d, a)},
$$

if, in the second equation, $a, b, c, d$ are in $\partial_{\infty} X-H[\infty]$. Note that each expression in the above two equalities is $-\infty$ if $a=c$ or $b=d$, and $+\infty$ if $c=b$ or $a=d$. If the points $\xi$ and $a$ coincide, the expression of the crossratio simplifies to

$$
[\xi, b, c, d]=\log \frac{d_{H}(b, d)}{d_{H}(c, b)} .
$$

The crossratio is continuous on $\partial_{4} X$, it is invariant under the diagonal action of the isometry group of $\Gamma$, and it has the following symmetries

$$
[c, d, a, b]=[a, b, c, d] \quad \text { and } \quad[a, b, d, c]=[b, a, c, d]=-[a, b, c, d] .
$$

If $X=\mathbb{H}_{\mathbb{R}}^{n}$ and $\xi$ is the point at infinity $\infty$ in the upper halfspace model of $\mathbb{H}_{\mathbb{R}}^{n}$, then the Hamenstädt distance coincides with a constant multiple of the Euclidean distance of $\partial_{\infty} \mathbb{H}_{\mathbb{R}}^{n}-\{\infty\}=\mathbb{R}^{n-1}$ (see for instance Hersonsky and Paulin [30]). In particular, if $n=2$ or $n=3$, then our crossratio is the logarithm of the modulus of the classical crossratio of four points in $\mathbb{C} \cup\{\infty\}$.

If $\xi_{0} \in \partial_{\infty} X-\partial_{\infty} L$, we define the crossratio penetration map $\mathfrak{c r p}_{L}: T_{\xi_{0}}^{1} X \rightarrow[0,+\infty]$ as follows. Let $\gamma$ be a geodesic line starting at $\gamma_{-}=\xi_{0}$, and ending at $\gamma_{+} \in \partial_{\infty} X$. Let $L_{1}, L_{2}$ be the endpoints of $L$. Set

$$
\mathfrak{c r p}_{L}(\gamma)=\max \left\{0,\left[\gamma_{-}, L_{1}, \gamma_{+}, L_{2}\right],\left[\gamma_{-}, L_{2}, \gamma_{+}, L_{1}\right]\right\} .
$$

Note that $\mathfrak{c r p}_{L}(\gamma)=+\infty$ if $\gamma_{+}$is equal to $L_{1}$ or $L_{2}$. The map $\mathfrak{c r p}_{L}$ is clearly continuous, and is independent of the ordering $L_{1}, L_{2}$ of the endpoints of $L$.

If $H$ is a horosphere centered at $\xi_{0}$, then

$$
\left[\xi_{0}, L_{1}, \gamma_{+}, L_{2}\right]=\log \frac{d_{H}\left(L_{1}, L_{2}\right)}{d_{H}\left(\gamma_{+}, L_{1}\right)},
$$

and the level sets for $\mathfrak{c r p}_{L}$ have a simple form: $\left[\xi_{0}, L_{1}, \gamma_{+}, L_{2}\right]=c$ if and only if $\gamma_{+}$is on the sphere of radius $e^{-c} d_{H}\left(L_{1}, L_{2}\right)$ centered at $L_{1}$ with respect to the Hamenstädt metric. Thus, in particular, the boundary of the zero set of $\mathfrak{c r p}_{L}$ is the boundary of the 
union of the two balls of radius $d_{H}\left(L_{1}, L_{2}\right)$ centered at $L_{1}$ and $L_{2}$. Furthermore, if $c>\log 2$, then the level set $\mathfrak{c r p}_{L}{ }^{-1}(c)$ is the union of two spheres for the Hamenstädt distance $d_{H}$ of centers $L_{1}$ and $L_{2}$ and radius $e^{-c} d_{H}\left(L_{1}, L_{2}\right)$. These two spheres are disjoint by the triangle inequality. Each of them separates $\xi_{0}$ from exactly one of the endpoints of $L$. We will use this in the proof of Lemma 3.9.

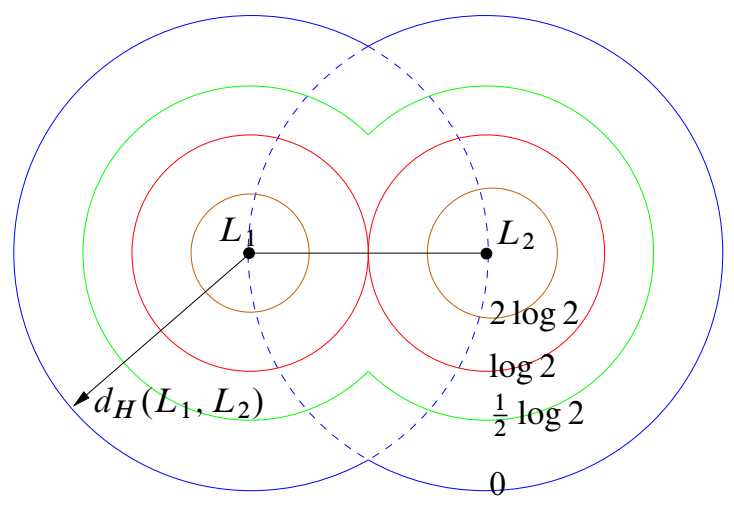

Note that if $X$ is a negatively curved symmetric space, then the spheres and balls of the Hamenstädt distance are topological spheres and balls in the topological sphere $\partial_{\infty} X$ (see Hersonsky and Paulin [30] if $X=\mathbb{H}_{\mathbb{R}}^{n}$ and Hersonsky and Paulin [31] if $X=\mathbb{H}_{\mathbb{C}}^{n}$ ). We do not know (and in fact we doubt it) whether this always holds in the general variable curvature case.

Lemma 3.5 Let $(a, b, c, d) \in \partial_{4} X$. If $b=d$, we define by convention $p=q=b$ and $d(p, q)=0$. Otherwise, let $p$ and $q$ be the closest points on $[b, d]$ of $a$ and $c$ respectively.

(1) If $b, q, p, d$ are in this order on $[b, d]$ and $d(p, q) \geq c_{1}^{\prime}(\infty)$, then $\mid[a, b, c, d]-$ $d(p, q) \mid \leq 2 c_{1}^{\prime}(\infty)$.

(2) If $b, p, q, d$ are in this order on $[b, d]$ and $d(p, q) \geq c_{1}^{\prime}(\infty)$, then $[a, b, c, d] \leq$ $c_{1}^{\prime}(\infty)$.

(3) If $d(p, q) \leq c_{1}^{\prime}(\infty)$, then $[a, b, c, d] \leq 2 c_{1}^{\prime}(\infty)$.

Proof If $a=d$ or $c=b$, then $p=d$ or $q=b$, hence we are in case (1) with $b \neq d$, and $[a, b, c, d]=d(p, q)=+\infty$, which proves the result. If $a=c$ or $b=d$, then $p=q$, we are in case (3) and $[a, b, c, d]=-\infty$, which proves the result. Hence we may assume that $a, b, c, d$ are pairwise disjoint.

Let $t \mapsto a_{t}, b_{t}, c_{t}, d_{t}$ be geodesic rays converging to respectively $a, b, c, d$ as $t \rightarrow \infty$, and let $p_{t}$ and $q_{t}$ be the closest points to $a_{t}$ and $c_{t}$ respectively on $\left[b_{t}, d_{t}\right]$. Let 
$p^{\prime} \in\left[a_{t}, d_{t}\right]$ and $p^{\prime \prime} \in\left[a_{t}, c_{t}\right]$ be the closest points to $p_{t}$ on $\left[a_{t}, d_{t}\right]$ and $\left[a_{t}, c_{t}\right]$, and let $q^{\prime} \in\left[b_{t}, c_{t}\right]$ and $q^{\prime \prime} \in\left[a_{t}, c_{t}\right]$ be the closest points to $q_{t}$ on $\left[b_{t}, c_{t}\right]$ and $\left[a_{t}, c_{t}\right]$.

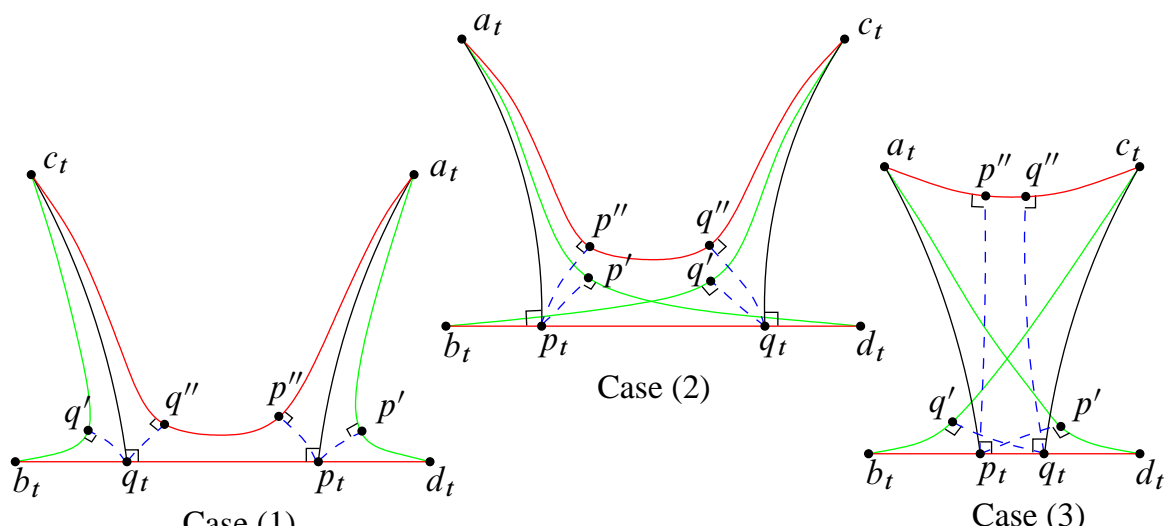

Case (1)

Case (3)

Recall that by an easy comparison argument, for pairwise distinct points $u, v, w$ in $X \cup \partial_{\infty} X$, if $r$ is the closest point to $w$ on $] u, v$ [, then $r$ is at distance less than $\delta=\log (1+\sqrt{2})$ from a point on $] u, w\left[\right.$. We will apply this remark to $r=p_{t}$ and $r=q_{t}$. Recall also that $c_{1}^{\prime}(\infty)=2 \delta$.

Case (1) Under the assumptions of Assertion (1), if $t$ is big enough, then the points $b_{t}, q_{t}, p_{t}, d_{t}$ are in this order on $\left[b_{t}, d_{t}\right]$. Using the triangle inequality on $d\left(a_{t}, c_{t}\right)$ and $d\left(b_{t}, d_{t}\right)$, and inserting the points $p^{\prime}$ and $q^{\prime}$ in $\left[d_{t}, a_{t}\right]$ and $\left[c_{t}, b_{t}\right]$, we have

$$
\begin{aligned}
d\left(a_{t}, c_{t}\right)- & d\left(c_{t}, b_{t}\right)+d\left(b_{t}, d_{t}\right)-d\left(d_{t}, a_{t}\right) \\
\leq & d\left(a_{t}, p_{t}\right)+d\left(p_{t}, q_{t}\right)+d\left(q_{t}, c_{t}\right)-d\left(c_{t}, q_{t}\right)-d\left(q_{t}, b_{t}\right)+2 d\left(q^{\prime}, q_{t}\right) \\
& +d\left(b_{t}, q_{t}\right)+d\left(q_{t}, p_{t}\right)+d\left(p_{t}, d_{t}\right)-d\left(d_{t}, p_{t}\right)-d\left(p_{t}, a_{t}\right)+2 d\left(p^{\prime}, p_{t}\right) \\
\leq & 2 d\left(p_{t}, q_{t}\right)+4 \delta .
\end{aligned}
$$

By comparison and a standard argument on hyperbolic quadrilaterals with three right angles [5, page 157], for every $\epsilon>0$, if $t$ is big enough, we have that $d\left(q_{t}, q^{\prime \prime}\right) \leq$ $2 \delta+\epsilon / 4$, as $d\left(p_{t}, q_{t}\right) \rightarrow d(p, q) \geq c_{1}^{\prime}(\infty)$. If we insert the points $p^{\prime \prime}$ and $q^{\prime \prime}$ in $\left[a_{t}, c_{t}\right]$, we get, as above,

$$
\begin{aligned}
d\left(a_{t}, c_{t}\right) & -d\left(c_{t}, b_{t}\right)+d\left(b_{t}, d_{t}\right)-d\left(d_{t}, a_{t}\right) \\
\geq & d\left(a_{t}, p_{t}\right)+d\left(p_{t}, q_{t}\right)+d\left(q_{t}, c_{t}\right)-2 d\left(p_{t}, p^{\prime \prime}\right)-2 d\left(q_{t}, q^{\prime \prime}\right)-d\left(c_{t}, q_{t}\right) \\
& -d\left(q_{t}, b_{t}\right)+d\left(b_{t}, q_{t}\right)+d\left(q_{t}, p_{t}\right)+d\left(p_{t}, d_{t}\right)-d\left(d_{t}, p_{t}\right)-d\left(p_{t}, a_{t}\right) \\
\geq & 2 d\left(p_{t}, q_{t}\right)-8 \delta+\epsilon .
\end{aligned}
$$

This proves Assertion (1) in Lemma 3.5. 
Case (2) The proof of Assertion (2) is almost identical to the one of the upper bound in the first inequality in Case (1). The different order of the points $p_{t}$ and $q_{t}$ now causes cancellations:

$$
\begin{aligned}
d\left(a_{t}, c_{t}\right)- & d\left(c_{t}, b_{t}\right)+d\left(b_{t}, d_{t}\right)-d\left(d_{t}, a_{t}\right) \\
\leq & d\left(a_{t}, p_{t}\right)+d\left(p_{t}, q_{t}\right)+d\left(q_{t}, c_{t}\right)-d\left(c_{t}, q_{t}\right)-d\left(q_{t}, p_{t}\right) \\
& -d\left(p_{t}, b_{t}\right)+2 d\left(q^{\prime}, q_{t}\right)+d\left(b_{t}, p_{t}\right)+d\left(p_{t}, q_{t}\right)+d\left(q_{t}, d_{t}\right)-d\left(d_{t}, q_{t}\right) \\
& -d\left(q_{t}, p_{t}\right)-d\left(p_{t}, a_{t}\right)+2 d\left(p^{\prime}, p_{t}\right) \\
\leq & 4 \delta .
\end{aligned}
$$

Case (3) Assume that $d(p, q) \leq c_{1}^{\prime}(\infty)$ and let $\epsilon>0$. By taking $t$ big enough, we can assume that $d\left(p_{t}, q_{t}\right) \leq 2 \delta+\epsilon$. Inserting the points $p^{\prime \prime}$ and $q^{\prime \prime}$ in $\left[a_{t}, c_{t}\right]$ and using the fact that closest point maps do not increase distances, we have $d\left(p^{\prime \prime}, q^{\prime \prime}\right) \leq 2 \delta+\epsilon$, $d\left(c_{t}, q_{t}\right) \geq d\left(c_{t}, q^{\prime \prime}\right)$ and $d\left(a_{t}, p_{t}\right) \geq d\left(a_{t}, p^{\prime \prime}\right)$. Thus, as in the cases above,

$$
\begin{aligned}
d\left(a_{t}, c_{t}\right)- & d\left(c_{t}, b_{t}\right)+d\left(b_{t}, d_{t}\right)-d\left(d_{t}, a_{t}\right) \\
\leq & d\left(a_{t}, p^{\prime \prime}\right)+d\left(p^{\prime \prime}, q^{\prime \prime}\right)+d\left(q^{\prime \prime}, c_{t}\right)-d\left(c_{t}, q_{t}\right)-d\left(q_{t}, b_{t}\right)+2 d\left(q^{\prime}, q_{t}\right) \\
& +d\left(b_{t}, q_{t}\right)+d\left(q_{t}, p_{t}\right)+d\left(p_{t}, d_{t}\right)-d\left(d_{t}, p_{t}\right)-d\left(p_{t}, a_{t}\right)+2 d\left(p^{\prime}, p_{t}\right) \\
\leq & 8 \delta+2 \epsilon .
\end{aligned}
$$

As this holds for any $\epsilon>0$, the result follows.

Lemma 3.6 Let $\epsilon>0$, let $L$ be a geodesic line in $X$, and assume that $\xi_{0} \in \partial_{\infty} X-$ $\partial_{\infty} L$. The map $\mathfrak{c r p}_{L}$ is a continuous $\left(2 c_{1}^{\prime}(\epsilon)+2 c_{1}^{\prime}(\infty)+2 \epsilon\right)$-penetration map in the $\epsilon$-convex set $\mathcal{N}_{\epsilon} L$ and $\left\|\mathfrak{c r p}_{L}-\mathfrak{f t p}\right\|_{L} \leq 2 c_{1}^{\prime}(\infty)$.

Proof Let $\gamma \in T_{\xi_{0}}^{1} X$, let $\gamma_{-}=\xi_{0}$ and $\gamma_{+}$be the endpoints of $\gamma$, and $L_{1}$ and $L_{2}$ be the endpoints of $L$. Let $p$ and $q$ be the closest points to $\gamma_{-}$and $\gamma_{+}$on $L$ respectively, so that $\mathfrak{f t p}_{L}(\gamma)=d(p, q)$.

If $d(p, q) \leq c_{1}^{\prime}(\infty)$, then Lemma 3.5 (3) implies that $0 \leq \mathfrak{c r p}_{L}(\gamma) \leq 2 c_{1}^{\prime}(\infty)$, and thus $\left|\mathfrak{c r p}_{L}(\gamma)-\mathfrak{f t p}_{L}(\gamma)\right| \leq 2 c_{1}^{\prime}(\infty)$.

If $d(p, q)>c_{1}^{\prime}(\infty)$, then up to renaming the endpoints of $L$, we have by Lemma 3.5 (2) and (1) that $\left[\gamma_{-}, L_{2}, \gamma_{+}, L_{1}\right] \leq c_{1}^{\prime}(\infty)$ and $-2 c_{1}^{\prime}(\infty)+\mathfrak{f t p}_{L}(\gamma) \leq\left[\gamma_{-}, L_{1}, \gamma_{+}, L_{2}\right] \leq$ $2 c_{1}^{\prime}(\infty)+\mathfrak{f t p}_{L}(\gamma)$, which implies the result, using Lemma 3.4.

Remark The penetration maps can be defined for any fixed starting point which is outside the $\epsilon$-convex set $C$ and its boundary at infinity, except for $\mathfrak{c r p}_{L}$. Thus, the penetration maps $\ell_{C}, \mathfrak{b p}_{C}, \mathfrak{p h}_{H}, \mathfrak{i p p}_{H}, \mathfrak{p h} \mathfrak{h}_{B}, \mathfrak{i p p}_{B}, \mathfrak{f t p _ { L }}$ considered in this section are 
all restrictions to $T_{\xi_{0}}^{1} X$ of maps defined, and continuous (as an inspection of the above proofs shows) on $\bigcup_{\xi \notin C^{\prime} \cup \partial_{\infty} C^{\prime}} T_{\xi}^{1} X \subset T^{1} X$ with $C^{\prime}$ respectively $C, C, H, H, B, B$, $\mathcal{N}_{\epsilon} L$. The penetration map $\mathfrak{c r p}_{L}$ is defined and continuous on $\bigcup_{\xi \in \partial_{\infty} X-\partial_{\infty} C^{\prime}} T_{\xi}^{1} X$. This point of view is used in cases (3) and (4) of Proposition 3.7 below, and will be useful to apply Corollary 4.11 .

\subsection{Prescribing the penetration}

In Section 4, we will use the following operation repeatedly: a geodesic ray or line $\gamma$ starting from a given point $\xi_{0}$ is given that penetrates two $\epsilon$-convex sets $C$ and $C^{\prime}$ with penetration maps $f$ and $f^{\prime}$, first entering $C$ with $f(\gamma)=h$, and then $C^{\prime}$ with $f^{\prime}(\gamma) \geq h^{\prime}$. We will need to pick a new geodesic ray or line $\gamma^{\prime}$ starting from $\xi_{0}$ which intersects $C$ before $C^{\prime}$, for which we still have $f\left(\gamma^{\prime}\right)=h$, and for which we now have the equality $f^{\prime}\left(\gamma^{\prime}\right)=h^{\prime}$. In the following result, we show that this operation is possible in a number of geometric cases. These cases will be used in Section 5 for various applications.

Proposition 3.7 Let $X$ be a complete, simply connected Riemannian manifold with sectional curvature at most -1 and dimension at least 3. Let $\epsilon>0$ and $\delta, h, h^{\prime} \geq 0$. Let $C$ and $C^{\prime}$ be $\epsilon$-convex subsets of $X$, and $\xi_{0} \in\left(X \cup \partial_{\infty} X\right)-\left(C \cup \partial_{\infty} C\right)$. Let $f$ and $f^{\prime}$ be maps $T_{\xi_{0}}^{1} X \rightarrow[0,+\infty]$, with $f^{\prime}$ continuous and $\kappa^{\prime}=\left\|f^{\prime}-\ell_{C^{\prime}}\right\|_{\infty}<+\infty$. Consider the following cases:

(1) $C$ is a horoball with $\operatorname{diam}\left(C \cap C^{\prime}\right) \leq \delta$; $f$ is either the penetration height map $\mathfrak{p h}_{C}$ or the inner-projection penetration map $\mathfrak{i p p}_{C}$;

$$
h \geq h^{\min }=2 c_{1}^{\prime}(\epsilon)+2 \delta+\left\|f-\mathfrak{p h}_{C}\right\|_{\infty}
$$

and $h^{\prime} \geq h_{0}^{\min }=\kappa^{\prime}+2 \delta$; if $C^{\prime}$ is also a horoball we may take $\epsilon=+\infty$ in the definition of $h^{\mathrm{min}}$.

(2) $C$ is a ball of radius $R(\geq \epsilon)$ with $\operatorname{diam}\left(C \cap C^{\prime}\right) \leq \delta$; $f$ is either the penetration height map $\mathfrak{p h}_{C}$ or the inner-projection penetration map $\mathfrak{i p p}_{C}$;

$$
h^{\min }=2 c_{1}^{\prime}(\epsilon)+2 \delta+\|f-\mathfrak{p h}\|_{C} \leq h \leq 2 R-2 c_{1}^{\prime}(\epsilon)-\|f-\mathfrak{p h}\|_{C} \|_{\infty}=h^{\max }
$$

and $h^{\prime} \geq h_{0}^{\min }=\kappa^{\prime}+2 \delta$;

(3) $C$ is the $\epsilon$-neighbourhood of a complete totally geodesic subspace $L$ of dimension at least 2 , with $\operatorname{diam}\left(C \cap C^{\prime}\right) \leq \delta$; either $f=\ell_{C}$ and $X$ has constant curvature, or $f$ is the fellow-traveller penetration map $\mathfrak{f t p}_{L}$;

$$
h \geq h^{\min }=4 c_{1}^{\prime}(\epsilon)+2 \epsilon+\delta+2\left\|f-\mathfrak{f t p}_{L}\right\|_{\infty}
$$

and $h^{\prime}>h_{0}^{\min }=\kappa^{\prime}+\delta$; 
(4) - $C$ is the $\epsilon$-neighbourhood of a geodesic line $L$;

- $h \geq h^{\min }=4 c_{1}^{\prime}(\epsilon)+2 \epsilon+\delta+\left\|f-\mathfrak{f t p}_{L}\right\|_{\infty}$;

- either $f=\ell_{C}$ and $X$ has constant curvature, or $f$ is the fellow-traveller penetration map $\mathfrak{f t p}_{L}$, or $\xi_{0} \in \partial_{\infty} X, f=\mathfrak{c r p}_{L}$, and the metric spheres of the Hamenstädt distance on $\partial_{\infty} X-\left\{\xi_{0}\right\}$ are topological spheres;

- either $C^{\prime}$ is any $\epsilon$-convex subset that does not meet $C$ (in which case $\delta=0$ ) and $h^{\prime}>h_{0}^{\min }=\kappa^{\prime}$, or $C^{\prime}$ is the $\epsilon$-neighbourhood of a totally geodesic subspace with codimension at least two such that $\operatorname{diam}\left(C \cap C^{\prime}\right) \leq \delta$ and

$$
h^{\prime} \geq h_{0}^{\min }=3 c_{1}^{\prime}(\epsilon)+3 \epsilon+\delta+\left\|f^{\prime}-\mathfrak{f t p}_{L^{\prime}}\right\|_{\infty} .
$$

Assume that one of the above cases holds. If there exists a geodesic ray or line $\gamma$ starting from $\xi_{0}$ which meets first $C$ and then $C^{\prime}$ with $f(\gamma)=h$ and $f^{\prime}(\gamma) \geq h^{\prime}$, then there exists a geodesic ray or line $\bar{\gamma}$ starting from $\xi_{0}$ which meets first $C$ and then $C^{\prime}$ with $f(\bar{\gamma})=h$ and $f^{\prime}(\bar{\gamma})=h^{\prime}$.

Proof Let $\gamma$ be as in the statement, and $x$ (resp. $y$ ) be the point where $\gamma$ enters (resp. exits) $C$ (with $y$ in $X$ since $f(\gamma)=h<+\infty$ ). Let $x^{\prime}$ (resp. $y^{\prime}$ ) be the point where $\gamma$ enters (resp. exits) $C^{\prime}$ (with $x^{\prime} \in X$ but possibly with $y^{\prime}$ at infinity). By convexity, $\xi_{0} \notin C^{\prime} \cup \partial_{\infty} C^{\prime}$. For every $h \geq 0$, we define $A$ as the set of points $\alpha(+\infty)$ where $\alpha \in T_{\xi_{0}}^{1} X$ satisfies $f(\alpha)=h$. Let $A_{0}$ be the arcwise connected component of $A$ containing $\gamma(+\infty)$. By considering the various cases, we will prove below the following two claims:

(a) Every geodesic ray or line, starting from $\xi_{0}$ and meeting $C^{\prime}$, first meets $C$ and then $C^{\prime}$.

(b) There exists a geodesic ray or line $\bar{\gamma}_{0}$ starting from $\xi_{0}$ with $\bar{\gamma}_{0}(+\infty)$ belonging to $A_{0}$, and $f^{\prime}\left(\bar{\gamma}_{0}\right) \leq h_{0}^{\min }$.

As $f^{\prime}$ is continuous and $A_{0}$ is arcwise connected, the intermediate value theorem implies the existence of a geodesic $\bar{\gamma}$ with the desired properties, and Proposition 3.7 is proven.

Case (1) Let $\kappa=\|f-\mathfrak{p h} C\|_{\infty}$. Let $\xi$ be the point at infinity of $C$, which is different from $\xi_{0}$, and let $p_{\xi}$ be the closest point to $\xi$ on $\gamma$. As $\mathfrak{p h}_{C}(\gamma) \geq f(\gamma)-\kappa=h-\kappa \geq$ $h^{\mathrm{min}}-\kappa>0$, the point $p_{\xi}$ belongs to the interior of the horoball $C$. Let $\gamma_{\xi}$ be the geodesic ray or line starting from $\xi_{0}$ with $\gamma_{\xi}(+\infty)=\xi$. 


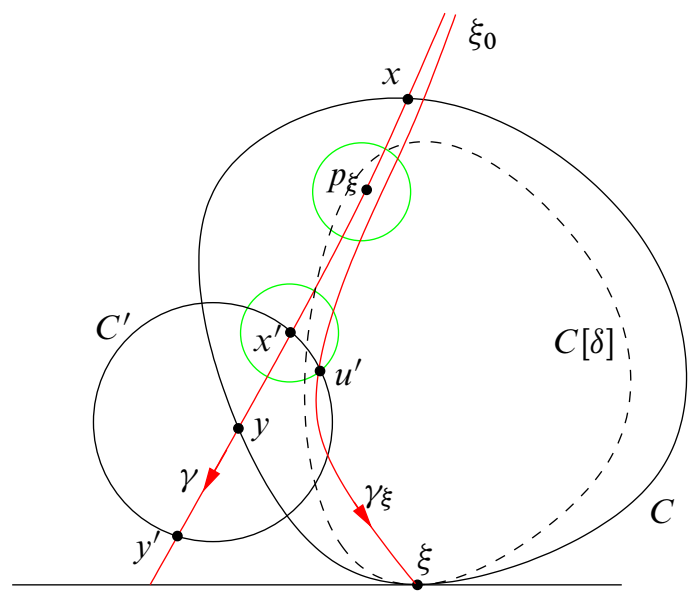

We start by proving the (stronger) first claim that every geodesic ray or line starting from $\xi_{0}$ and meeting $C^{\prime}$ meets $C[\delta]$ first (hence it meets $C$ before $C^{\prime}$ and Assertion (a) holds). Note that

$d\left(y, p_{\xi}\right) \geq d\left(p_{\xi}, \partial C\right)=\frac{\mathfrak{p h}_{C}(\gamma)}{2} \geq \frac{f(\gamma)-\kappa}{2}=\frac{h-\kappa}{2} \geq \frac{h^{\min }-\kappa}{2}=c_{1}^{\prime}(\epsilon)+\delta>\delta$.

As $f^{\prime}(\gamma) \geq h^{\prime} \geq h_{0}^{\min }=\kappa^{\prime}+2 \delta$, we have $\ell_{C^{\prime}}(\gamma) \geq f^{\prime}(\gamma)-\kappa^{\prime}>\delta$, unless $\ell_{C^{\prime}}(\gamma)=\delta=0$. Note that $\gamma \cap C^{\prime}$ is not contained in the geodesic segment $[x, y]$. Otherwise, this would contradict the assumption that $\operatorname{diam}\left(C \cap C^{\prime}\right) \leq \delta$ when $\ell_{C^{\prime}}(\gamma)>\delta$. When $\ell_{C^{\prime}}(\gamma)=\delta=0$, as $\gamma$ meets $C^{\prime}$, the segment $\gamma \cap C^{\prime}$ would be reduced to a point by the convexity of $C^{\prime}$. This point would be $\{x\}$ or $\{y\}$ (as $C^{\prime}$ is not a singleton). But then the tangent vector of $\gamma$ at $x$ or its opposite at $y$ would both enter strictly $C$ and be tangent to $C^{\prime}$, which contradicts the fact that $\delta=0$.

As $\gamma$ meets $C$ before $C^{\prime}$, this implies, in particular, that the geodesic ray $[y, \gamma(+\infty)[$ meets $C^{\prime}$, and that the point $p_{\xi}$ belongs to $\left.] x^{\prime}, \xi_{0}\right]$ : otherwise $C \cap C^{\prime}$ would contain a segment of length at least $d\left(p_{\xi}, y\right)>\delta$, which is impossible. Hence, by convexity, any geodesic ray or line starting from $\xi_{0}$ and meeting $B\left(x^{\prime}, c_{1}^{\prime}(\epsilon)\right)$ first meets $B\left(p_{\xi}, c_{1}^{\prime}(\epsilon)\right)$. By Lemma 2.3, every geodesic ray or line, starting from $\xi_{0}$ and meeting $C^{\prime}$, meets the ball $B\left(x^{\prime}, c_{1}^{\prime}(\epsilon)\right)$ before entering $C^{\prime}$ (and we may take $\epsilon=+\infty$ if $C^{\prime}$ is also a horoball, by Lemma 2.9). This proves the first claim, as the ball $B\left(p_{\xi}, c_{1}^{\prime}(\epsilon)\right)$ is contained in $C[\delta]$, since $d\left(p_{\xi}, \partial C\right) \geq c_{1}^{\prime}(\epsilon)+\delta$, as seen above.

Let us prove now the (stronger) second claim that there exists a geodesic ray or line $\bar{\gamma}_{0}$ starting from $\xi_{0}$ with $\bar{\gamma}_{0}(+\infty)$ belonging to $A_{0}$, and avoiding the interior of $C^{\prime}$, which implies Assertion (b), as then $f^{\prime}\left(\bar{\gamma}_{0}\right) \leq \ell_{C^{\prime}}\left(\bar{\gamma}_{0}\right)+\kappa^{\prime}=\kappa^{\prime} \leq h_{0}^{\min }$. 
The subspace $A$ of $\partial_{\infty} X$ is a codimension 1 topological submanifold of the topological sphere $\partial_{\infty} X$, which is homeomorphic to the sphere $\mathbb{S}^{n-2}$, hence it is arcwise connected. Indeed, if $f=\mathfrak{p h}_{C}$, then $A$ is the subset of endpoints of the geodesic rays or lines starting from $\xi_{0}$ that are tangent to $\partial(C[h / 2])$. If $f=\mathfrak{i p p}_{C}$, the subset $A$ is the preimage of a point in $] \xi_{0}, \xi$ [ by the closest point map from $\partial_{\infty} X$ to $\left[\xi_{0}, \xi\right]$, which is, over $] \xi_{0}, \xi$ [, a trivial topological bundle with fibers homeomorphic to $\mathbb{S}^{n-2}$.

Note that $f$ is continuous, $f\left(\gamma_{\xi}\right)=\infty>h$, and $f(\alpha)=0$ if $\alpha$ is a geodesic ray or line starting from $\xi_{0}$ with $\alpha(+\infty)$ close enough to $\gamma(-\infty)$. Therefore $A$ separates $\gamma(-\infty)$ and $\gamma_{\xi}(+\infty)$, as the connected components of $\partial_{\infty} X-A$ are arcwise connected.

If the (stronger) second claim is not true, then the topological sphere $A_{0}=A$ of dimension $n-2$ is contained in the interior of the shadow $\mathcal{O}_{\xi_{0}} C^{\prime}$. As $\xi_{0} \notin C^{\prime} \cup \partial_{\infty} C^{\prime}$, this shadow is homeomorphic to a ball of dimension $n-1$. Thus, by Jordan's theorem, one of the two connected components of $\partial_{\infty} X-A$ is contained in the interior of $\mathcal{O}_{\xi_{0}} C^{\prime}$. As $\gamma(-\infty)$ does not belong to $\mathcal{O}_{\xi_{0}} C^{\prime}$ and $A$ separates $\gamma(-\infty)$ and $\gamma_{\xi}(+\infty)$, this implies that $\gamma_{\xi}(+\infty)$ belongs to the interior of $\mathcal{O}_{\xi_{0}} C^{\prime}$. Hence $\gamma_{\xi}$ meets the interior of $C^{\prime}$.

Therefore, by the first claim, the geodesic ray or line $\gamma_{\xi}$ meets $C[\delta]$ before meeting $C^{\prime}$. Let $u^{\prime}$ be the entering point of $\gamma_{\xi}$ in $C^{\prime}$. As $\xi$ is the point at infinity of $C[\delta]$, the points $\xi_{0}, u^{\prime}, \xi$ are in this order on $\gamma_{\xi}$. Hence by convexity, this implies that $u^{\prime}$ belongs to $C[\delta]$. As $f^{\prime}(\gamma) \geq h^{\prime} \geq h_{0}^{\min }=\kappa^{\prime}+2 \delta$, we have

$$
d\left(x^{\prime}, y^{\prime}\right)=\ell_{C^{\prime}}(\gamma) \geq f^{\prime}(\gamma)-\kappa^{\prime} \geq 2 \delta .
$$

Hence by the triangle inequality, one of the two distances $d\left(u^{\prime}, x^{\prime}\right), d\left(u^{\prime}, y^{\prime}\right)$ is at least $\delta$, and by the strict convexity of the distance, it is strictly bigger than $\delta$ (as $u^{\prime}$ does not belong to $\gamma\left(\right.$ as $\left.\gamma \neq \gamma_{\xi}\right)$ ). Hence, if $u^{\prime \prime}$ is a point close enough to $u^{\prime}$ in $] u^{\prime}, \xi$, then $u^{\prime \prime}$ belongs to the interior of $C^{\prime}$ and to the interior of $C[\delta]$, and is at distance strictly greater than $\delta$ from either $z^{\prime}=x^{\prime}$ or $z^{\prime}=y^{\prime}$. Therefore, the geodesic segment $\left[u^{\prime \prime}, z^{\prime}\right] \cap C$ has length strictly bigger than $\delta$, and is contained in the intersection $C \cap C^{\prime}$. This contradicts the assumption that $\operatorname{diam}\left(C \cap C^{\prime}\right) \leq \delta$.

Case (2) The proof is completely similar to Case (1). Let now $z$ be the center of the ball $C$, let $p_{z}$ be the point of $\gamma$ the closest to $z$, let $\gamma_{z}$ be the geodesic ray or line starting from $\xi_{0}$ and passing through $z$, and let $\kappa=\left\|f-\mathfrak{p h}_{C}\right\|_{\infty}$. Note that $R>h^{\max } / 2 \geq h^{\min } / 2 \geq \delta$, so that $C[\delta]$ is nonempty. We only have to replace $\xi$ by $z$, $p_{\xi}$ by $p_{z}$ and $\gamma_{\xi}$ by $\gamma_{z}$, and to replace two arguments in the above proof, the one in order to show that $A$ separates $\gamma(-\infty)$ from $\gamma_{z}(+\infty)$, and the one in order to show that $\xi_{0}, u^{\prime}, z$ are in this order on $\gamma_{z}$, where $u^{\prime}$ is the entering point of $\gamma_{z}$ in $C^{\prime}$. 
To prove that $A$ separates $\gamma(-\infty)$ from $\gamma_{z}(+\infty)$, we simply use now that $f\left(\gamma_{z}\right)=$ $2 R>h^{\max } \geq h$ instead of $f\left(\gamma_{\xi}\right)=\infty>h$. Let us prove that $\xi_{0}, u^{\prime}, z$ are in this order on $\gamma_{z}$. We have

$$
d\left(z, p_{z}\right)=R-\frac{\mathfrak{p h} C(\gamma)}{2} \geq R-\frac{f(\gamma)+\kappa}{2} \geq R-\frac{h^{\max }+\kappa}{2}=c_{1}^{\prime}(\epsilon) .
$$

By Lemma 2.3, we have $d\left(x^{\prime}, u^{\prime}\right) \leq c_{1}^{\prime}(\epsilon)$. As $\gamma_{z}$ meets the interior of $C^{\prime}$, by the same argument as in Case 1, we even have $d\left(u^{\prime}, \gamma\right)<c_{1}^{\prime}(\epsilon)$. Hence by strict convexity, we do have $\left.u^{\prime} \in\right] z, \xi_{0}$ [. The rest of the argument in the proof of Case (1) is unchanged.

Before studying the last two cases, we start by proving two lemmas. The first one implies the first of the two claims we need to prove in Cases (3), (4), and the second one gives the topological information on $A$ that we will need in these last two cases.

Lemma 3.8 Let $L$ be a complete totally geodesic subspace with dimension at least $1, \epsilon>0, C=\mathcal{N}_{\epsilon} L, \xi_{0} \in\left(X \cup \partial_{\infty} X\right)-\left(C \cup \partial_{\infty} L\right)$, and $C^{\prime}$ be an $\epsilon$-convex subset of $X$ such that $\operatorname{diam}\left(C \cap C^{\prime}\right) \leq \delta$. Let $f, f^{\prime}: T_{\xi_{0}}^{1} X \rightarrow[0,+\infty]$ be maps such that $\kappa=\left\|f-\mathfrak{f t p}_{L}\right\|_{\infty}<+\infty, \kappa^{\prime}=\left\|f^{\prime}-\ell_{C^{\prime}}\right\|_{\infty}<+\infty$. Let $\gamma$ be a geodesic ray or line starting from $\xi_{0}$, entering $C$ before entering $C^{\prime}$, such that $4 c_{1}^{\prime}(\epsilon)+2 \epsilon+\delta+\kappa \leq$ $f(\gamma)<+\infty$ and $f^{\prime}(\gamma)>\delta+\kappa^{\prime}$. If $\tilde{\gamma}$ is a geodesic ray or line starting from $\xi_{0}$ which meets $C^{\prime}$, then $\tilde{\gamma}$ meets the interior of $C$ before meeting $C^{\prime}$.

Proof Note that $\xi_{0} \notin C^{\prime} \cup \partial_{\infty} C^{\prime}$, by convexity and the assumptions on $\gamma$, as $\xi_{0} \notin$ $C \cup \partial_{\infty} C$. Let $L_{0}$ be the geodesic line passing through the closest points $p_{\xi_{0}}, p_{\gamma}(+\infty)$ on $L$ of $\xi_{0}, \gamma(+\infty)$, respectively. Note that

$$
d\left(p_{\xi_{0}}, p_{\gamma(+\infty)}\right)=\mathfrak{f t p}_{L}(\gamma) \geq f(\gamma)-\kappa \geq 4 c_{1}^{\prime}(\epsilon)+2 \epsilon+\delta>0 .
$$

Hence, by Lemma 3.4, and as $\mathfrak{f t p}_{L_{0}}(\gamma)=\mathfrak{f} \mathfrak{t p}_{L}(\gamma)$, we have

$$
\ell_{\mathcal{N}_{\epsilon} L_{0}}(\gamma) \geq \mathfrak{f t p}_{L_{0}}(\gamma)-2 c_{1}^{\prime}(\epsilon)-2 \epsilon>0 .
$$

In particular, $\gamma$ enters $\mathcal{N}_{\epsilon} L_{0}$ at a point $x_{0}$ and exits it at a point $y_{0}$ in $X$ (as $\gamma(+\infty) \notin$ $\partial_{\infty} L$ since $\left.f(\gamma)<+\infty\right)$. Let $u \mapsto p_{u}$ be the closest point map from $X \cup \partial_{\infty} X$ onto $L_{0} \cup \partial_{\infty} L_{0}$. Recall that this map does not increase the distances (and even decreases them, unless the two points under consideration coincide or are on $L_{0}$ ), and that it preserves betweenness, that is, if $u^{\prime \prime} \in\left[u, u^{\prime}\right]$, then $p_{u^{\prime \prime}} \in\left[p_{u}, p_{u^{\prime}}\right]$. Let $x^{\prime}$ (resp. $\tilde{x}^{\prime}$ ) be the point where $\gamma$ (resp. $\tilde{\gamma}$ ) enters $C^{\prime}$, and $q_{\xi_{0}}$ and $q_{\gamma(+\infty)}$ be the closest point to $\xi_{0}$ and $\gamma(+\infty)$ respectively on $\mathcal{N}_{\epsilon} L_{0}$. 


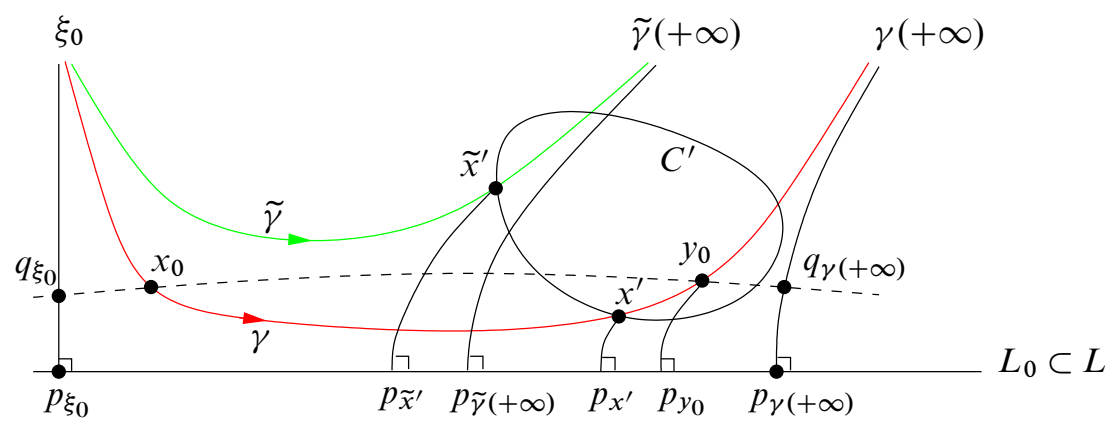

Recall that by Lemma 2.3, the distances $d\left(\tilde{x}^{\prime}, x^{\prime}\right), d\left(x_{0}, q_{\xi_{0}}\right), d\left(y_{0}, q_{\gamma(+\infty)}\right)$ are at most $c_{1}^{\prime}(\epsilon)$. Note that $\tilde{x}^{\prime} \in\left[\xi_{0}, \tilde{\gamma}(+\infty)\right]$. Hence, as betweenness is preserved,

$$
\begin{aligned}
\mathfrak{f t p}_{L_{0}}(\tilde{\gamma}) & =d\left(p_{\xi_{0}}, p_{\widetilde{\gamma}(+\infty)}\right) \geq d\left(p_{\xi_{0}}, p_{\widetilde{x}^{\prime}}\right) \geq d\left(p_{\xi_{0}}, p_{x^{\prime}}\right)-d\left(p_{x^{\prime}}, p_{\widetilde{x}^{\prime}}\right) \\
& \geq d\left(p_{\xi_{0}}, p_{x^{\prime}}\right)-d\left(x^{\prime}, \widetilde{x}^{\prime}\right) \geq d\left(p_{\xi_{0}}, p_{x^{\prime}}\right)-c_{1}^{\prime}(\epsilon) .
\end{aligned}
$$

Note that $d\left(p_{\xi_{0}}, p_{x^{\prime}}\right) \geq d\left(p_{\xi_{0}}, p_{y_{0}}\right)$ when $\xi_{0}, y_{0}, x^{\prime}$ are in this order on $\gamma$. When $\xi_{0}, y_{0}, x^{\prime}$ are not in this order on $\gamma$, as $\gamma$ enters in $C$ before $C^{\prime}$, as $\ell_{C^{\prime}}(\gamma) \geq f^{\prime}(\gamma)-$ $\kappa^{\prime}>\delta$ and as $\operatorname{diam}\left(C \cap C^{\prime}\right) \leq \delta$, we have $d\left(x^{\prime}, y_{0}\right) \leq \delta$; hence

$d\left(p_{\xi_{0}}, p_{x^{\prime}}\right) \geq d\left(p_{\xi_{0}}, p_{y_{0}}\right)-d\left(p_{y_{0}}, p_{x^{\prime}}\right) \geq d\left(p_{\xi_{0}}, p_{y_{0}}\right)-d\left(y_{0}, x^{\prime}\right) \geq d\left(p_{\xi_{0}}, p_{y_{0}}\right)-\delta$.

Therefore, in both cases, as $y_{0} \in\left[\xi_{0}, \gamma(+\infty)\right]$ and $u \mapsto p_{u}$ preserves the betweenness, and since $p_{\gamma(+\infty)}=p_{q_{\gamma(+\infty)}}$, we have, using (13),

$$
\begin{aligned}
\mathfrak{f t p}_{L_{0}}(\tilde{\gamma}) & \geq d\left(p_{\xi_{0}}, p_{\tilde{x}^{\prime}}\right) \geq d\left(p_{\xi_{0}}, p_{x^{\prime}}\right)-c_{1}^{\prime}(\epsilon) \geq d\left(p_{\xi_{0}}, p_{y_{0}}\right)-\delta-c_{1}^{\prime}(\epsilon) \\
& \geq d\left(p_{\xi_{0}}, p_{\gamma(+\infty)}\right)-d\left(p_{q_{\gamma(+\infty)}}, p_{y_{0}}\right)-c_{1}^{\prime}(\epsilon)-\delta \\
& >\mathfrak{f t p}_{L}(\gamma)-d\left(q_{\gamma(+\infty)}, y_{0}\right)-c_{1}^{\prime}(\epsilon)-\delta \\
& \geq \mathfrak{f t p}_{L}(\gamma)-2 c_{1}^{\prime}(\epsilon)-\delta \geq 2 c_{1}^{\prime}(\epsilon)+2 \epsilon
\end{aligned}
$$

By Lemma 3.4, we hence have

$$
\ell_{\mathcal{N}_{\epsilon} L}(\tilde{\gamma}) \geq \ell_{\mathcal{N}_{\epsilon} L_{0}}(\tilde{\gamma}) \geq \mathfrak{f} \mathfrak{t p}_{L_{0}}(\tilde{\gamma})-2 c_{1}^{\prime}(\epsilon)-2 \epsilon>0 .
$$

In particular, $\tilde{\gamma}$ does enter the interior of $C$, at a point $\tilde{x}$. Note that the geodesic from $\xi_{0}$ through $p_{\xi_{0}}$ enters $C$ at $q_{\xi_{0}}$. Now by contradiction, if $\tilde{\gamma}$ enters the interior of $C$ after it enters $C^{\prime}$, then $\tilde{x}^{\prime} \in\left[\xi_{0}, \tilde{x}\right]$, so that

$$
c_{1}^{\prime}(\epsilon) \geq d\left(q_{\xi_{0}}, \tilde{x}\right) \geq d\left(p_{\xi_{0}}, p_{\tilde{x}}\right) \geq d\left(p_{\xi_{0}}, p_{\tilde{x}^{\prime}}\right)>2 c_{1}^{\prime}(\epsilon)+2 \epsilon,
$$

as seen above, a contradiction. 
Lemma 3.9 Let $X$ be a complete, simply connected Riemannian manifold with sectional curvature at most -1 and dimension at least 3. Let $\epsilon, h>0$. Let $L$ be a complete totally geodesic submanifold with dimension at least 1 and $\xi_{0} \in$ $\left(X \cup \partial_{\infty} X\right)-\left(\mathcal{N}_{\epsilon} L \cup \partial_{\infty} L\right)$. Assume either that

(1) $f=\ell_{\mathcal{N}_{\epsilon} L}, X$ has constant curvature and $h \in\left[4 c_{1}^{\prime}(\epsilon)+2 \epsilon,+\infty[\right.$,

(2) $\left.\xi_{0} \in \partial_{\infty} X, \operatorname{dim} L=1, f=\mathfrak{c r p}_{L}, h \in\right] \log 2,+\infty[$, and the metric spheres of the Hamenstädt distance on $\partial_{\infty} X-\left\{\xi_{0}\right\}$ are topological spheres, or

(3) $f=\mathfrak{f t p}_{L}$.

Then

$$
A=\left\{\alpha(+\infty): \alpha \in T_{\xi_{0}}^{1} X, f(\alpha)=h\right\}
$$

is a codimension 1 topological submanifold of the topological sphere $\partial_{\infty} X$, which is homeomorphic to the torus $\mathbb{S}^{\operatorname{dim} L-1} \times \mathbb{S}^{\operatorname{codim} L-1}$. Furthermore:

(a) If $\operatorname{dim} L=1$, then $A$ has two arcwise connected components, homeomorphic to a sphere of dimension $n-2$. If $f=\mathfrak{c r p}_{L}$ or if $h>c_{1}^{\prime}(\epsilon)$, then each of them separates $\gamma(-\infty)$ and exactly one of the two points at infinity of $L$, for every geodesic ray or line $\gamma$ starting from $\xi_{0}$ if $f=\mathfrak{c r p}_{L}$, and for those meeting $\mathcal{N}_{\epsilon} L$ if $f \neq \mathfrak{c r p}_{L}$.

(b) If $\operatorname{codim} L=1$, then $A$ has two arcwise connected components, homeomorphic to a sphere of dimension $n-2$, separated by $\partial_{\infty} L$.

(c) If $\operatorname{dim} L \geq 2$ and $\operatorname{codim} L \geq 2$, then $A$ is arcwise connected.

In cases (b) and (c), for every component $A_{0}$ of $A$, for every geodesic ray $\rho$ in $L$ with $\rho(0)$ the closest point to $\xi_{0}$ on $L$, there exists $\eta \in A_{0}$ such that $\rho(h)$ is at distance at most $\left\|f-\mathfrak{f t p}_{L}\right\|_{\infty}$ from the closest point to $\eta$ on $\rho$.

Proof Let $\pi_{L}: X \cup \partial_{\infty} X \rightarrow L \cup \partial_{\infty} L$ be the closest point map, and $p_{0}=\pi_{L}\left(\xi_{0}\right)$. Note that $L$ has codimension at least one, by the existence of $\xi_{0}$.

Assume first that $f=\mathfrak{f t p}_{L}$. As $h>0$, the subspace $A$ of $\partial_{\infty} X$ is the preimage of the sphere (of dimension $\operatorname{dim} L-1$ ) of center $p_{0}$ and radius $h$ in $L$, by $\pi_{L}$. As $\pi_{L}: \partial_{\infty} X \backslash \partial_{\infty} L \rightarrow L$ is a trivial topological bundle whose fibers are spheres of dimension codim $L-1$, the topological structure (including the assertions (b) and (c)) of $A$ is immediate. The final statement on (b) and (c) is trivial as, by definition, $\rho(h)$ is the closest point to some point in $A_{0}$.

If $\operatorname{dim} L=1$ and $h>c_{1}^{\prime}(\epsilon)$, if $\gamma \in T_{\xi_{0}}^{1} X$ meets $\mathcal{N}_{\epsilon} L$, then by Lemma 2.3 and by convexity, $d\left(\pi_{L}(\gamma(-\infty)), p_{0}\right) \leq c_{1}^{\prime}(\epsilon)<h$. Hence, the separation statement in (a) follows. 
Assume then that $f=\mathfrak{c r p}_{L}$, and that the hypotheses of (2) are satisfied. The result in this case (only assertion (a) needs to be checked) follows from the discussion before Lemma 3.5.

Assume now that $f=\ell_{\mathcal{N}_{\epsilon} L}, X$ has constant curvature, and $h \in\left[4 c_{1}^{\prime}(\epsilon)+2 \epsilon,+\infty[\right.$. Let $S_{0}=\pi_{L}^{-1}\left(p_{0}\right) \cap \partial_{\infty} X$. Using normal coordinates along $L$, the topological sphere $\partial_{\infty} X$ is homeomorphic to the topological join of the spheres $\partial_{\infty} L$ of dimension $\operatorname{dim} L-1$ and $S_{0}$ of dimension $\operatorname{codim} L-1$

$$
S_{0} \vee \partial_{\infty} L=\left(S_{0} \times[0,+\infty] \times \partial_{\infty} L\right) / \sim,
$$

where $\sim$ is the equivalence relation generated by $(a, 0, b) \sim\left(a, 0, b^{\prime}\right)$ as well as $(a,+\infty, b) \sim\left(a^{\prime},+\infty, b\right)$, for every $a, a^{\prime}$ in $S_{0}$ and $b, b^{\prime}$ in $\partial_{\infty} L$. We denote by $[a, t, b]$ the equivalence class of $(a, t, b)$. We choose the parametrization of $\partial_{\infty} X$ by $S_{0} \vee \partial_{\infty} L$ such that $[a, 0, b]=a,[a,+\infty, b]=b, d\left(\pi_{L}([a, t, b]), p_{0}\right)=t$, and the geodesic rays $\left[\pi_{L}([a, t, b]),[a, t, b]\left[\right.\right.$ are parallel transports of $\left[p_{0}, a[\right.$ along the geodesic ray $\left[p_{0}, b[\right.$, for $0<t<+\infty$.

For every $t$ in $] 0,+\infty\left[\right.$ and every $(a, b)$ in $S_{0} \times \partial_{\infty} L$, let $\gamma_{[a, t, b]}$ be the geodesic ray or line starting from $\xi_{0}$ and ending at $[a, t, b]$. By the proof of Lemma 3.4 and by Lemma 3.2, we have

$$
-2 c_{1}^{\prime}(\epsilon) \leq \ell_{\mathcal{N}_{\epsilon} L}(\alpha)-\mathfrak{f t p}_{L}(\alpha) \leq 2 c_{1}^{\prime}(\epsilon)+2 \epsilon,
$$

for every $\alpha \in T_{\xi_{0}}^{1} X$. In particular, if $t=\mathfrak{f t p}_{L}\left(\gamma_{[a, t, b]}\right)>2 c_{1}^{\prime}(\epsilon)$, then $\ell_{\mathcal{N}_{\epsilon} L}\left(\gamma_{[a, t, b]}\right)>0$, that is $\gamma_{[a, t, b]}$ meets the interior of $\mathcal{N}_{\epsilon} L$. The points $p_{0}, a, b, \xi_{0}$ are contained in an isometrically embedded copy of $\mathbb{H}_{\mathbb{R}}^{3}$ in $X$, and therefore we can restrict to the case when $X$ is the upper halfspace model of $\mathbb{H}_{\mathbb{R}}^{3}$ and $L$ has dimension 1. If we normalize so that $\xi_{0}=\infty$ and the endpoints of $L$ are $b=1$ and $-b$, then the level sets of $\ell_{\mathcal{N}_{\epsilon} L}$ for positive values are drawn in the following picture. The level set $\ell_{\mathcal{N}_{\epsilon} L}^{-1}(2 \epsilon)$ is the unique figure-8 curve. Each level set $\ell_{\mathcal{N}_{\epsilon} L}^{-1}(t)$ for $t>2 \epsilon$ has exactly two components, one in each bounded component of the complement of $\ell_{\mathcal{N}_{\epsilon} L}^{-1}(2 \epsilon)$.

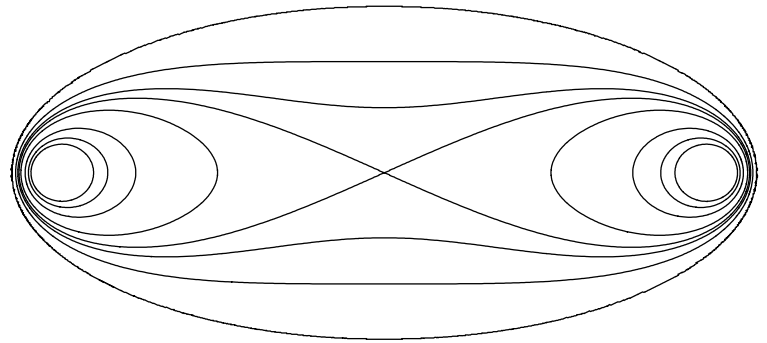


The curve $t \mapsto[a, t, b], t \geq 0$, is a segment of a circle through $b$ and $-b$ that connects the point $a$ on the imaginary axis to $b$. Thus, it is easy to see that the map from $\left[2 c_{1}^{\prime}(\epsilon),+\infty\left[\right.\right.$ to $\left[0,+\infty\left[\right.\right.$ defined by $t \mapsto \ell_{\mathcal{N}_{\epsilon} L}\left(\gamma_{[a, t, b]}\right)$ is continuous and strictly increasing, for every fixed $(a, b)$ in $S_{0} \times \partial_{\infty} L$.

Hence, as Lemma 3.4 gives

$$
\ell_{\mathcal{N}_{\epsilon} L}\left(\gamma_{\left[a, 2 c_{1}^{\prime}(\epsilon), b\right]}\right) \leq 4 c_{1}^{\prime}(\epsilon)+2 \epsilon \leq h<+\infty,
$$

there exists a unique $t_{a, b} \in\left[2 c_{1}^{\prime}(\epsilon),+\infty\right.$ [, depending continuously on $(a, b)$, such that $\ell_{\mathcal{N}_{\epsilon} L}\left(\gamma_{\left[a, t_{a, b}, b\right]}\right)=h$. In particular, the subset of points of $\partial_{\infty} X$ of the form $\left[a, t_{a, b}, b\right]$ for some $(a, b)$ in $S_{0} \times \partial_{\infty} L$ is indeed a codimension 1 topological submanifold of $\partial_{\infty} X$, which is homeomorphic to the torus $\mathbb{S}^{\operatorname{dim} L-1} \times \mathbb{S}^{\operatorname{codim} L-1}$. The statements (b) and (c) follow.

If $L$ has dimension 1 , and if $\gamma \in T_{\xi_{0}}^{1} X$ meets $\mathcal{N}_{\epsilon} L$, then by Lemma 2.3 and by convexity, $d\left(\pi_{L}(\gamma(-\infty)), p_{0}\right) \leq c_{1}^{\prime}(\epsilon)$. For every $\xi$ in a component $A_{0}$ of $A$, if as above $\xi=\left[a, t_{a, b}, b\right]$, then we have $d\left(\pi_{L}(\xi), p_{0}\right)=t_{a, b} \geq 2 c_{1}^{\prime}(\epsilon)$, hence $A_{0}$ separates $\gamma(-\infty)$ and $b$. This proves (a).

Let us prove the last assertion of the lemma. Let $\kappa=\left\|f-\mathfrak{f t p}_{L}\right\|_{\infty}$, and let $A_{0}$ be a connected component of $A$. For every $u$ in $L$ such that $d\left(u, p_{0}\right)=h$, let $\eta_{0}=[a, h, b]$, on the same side of $\partial_{\infty} L$ as $A_{0}$ if $\operatorname{codim} L=1$, be such that $\pi_{L}\left(\eta_{0}\right)=u$. Let $\eta_{t}=[a, h+t, b]$, which is on the same side of $\partial_{\infty} L$ as $A_{0}$ if $\operatorname{codim} L=1$. Note that

$$
f\left(\gamma_{[a, h+\kappa, b]}\right) \geq \mathfrak{f t p}_{L}\left(\gamma_{[a, h+\kappa, b]}\right)-\kappa=h,
$$

and similarly, $f\left(\gamma_{[a, h-\kappa, b]}\right) \leq h$. By the intermediate value theorem, there exists $t \in[-\kappa,+\kappa]$ such that $\eta_{t} \in A_{0}$. Hence $d\left(u, \pi_{L}\left(\eta_{t}\right)\right)=|t| \leq \kappa$.

Now we proceed with the proof of the remaining parts of Proposition 3.7.

Case (3) By Lemma 3.8, we only have to prove the second claim, that there exists a geodesic ray or line $\bar{\gamma}_{0}$ starting from $\xi_{0}$ with $\bar{\gamma}_{0}(+\infty)$ belonging to $A_{0}$, such that $f^{\prime}\left(\bar{\gamma}_{0}\right) \leq h_{0}^{\min }$.

Let $\kappa=\left\|f-\mathfrak{f t p}_{L}\right\|_{\infty}$. Let $p_{0}$ (respectively $p_{\gamma}$ ) be the point of $L$ the closest to $\xi_{0}$ (respectively $\gamma(+\infty)$ ), so that, in particular,

$$
d\left(p_{0}, p_{\gamma}\right)=\mathfrak{f t p}_{L}(\gamma) \geq f(\gamma)-\kappa=h-\kappa>0 .
$$

Let $p_{\gamma}^{\prime}$ be the point on the geodesic line $L_{0}$ (contained in $L$ ) passing through $p_{0}$ and $p_{\gamma}$ on the opposite side of $p_{\gamma}$ with respect to $p_{0}$, and at distance $h$ from $p_{0}$. By Lemma 3.9, there exists a geodesic line $\bar{\gamma}_{0}$ starting from $\xi_{0}$ and ending at a point in $A_{0}$ whose closest point $p_{\bar{\gamma}_{0}}$ on $L$ is at distance at most $\kappa$ from $p_{\gamma}^{\prime}$. 


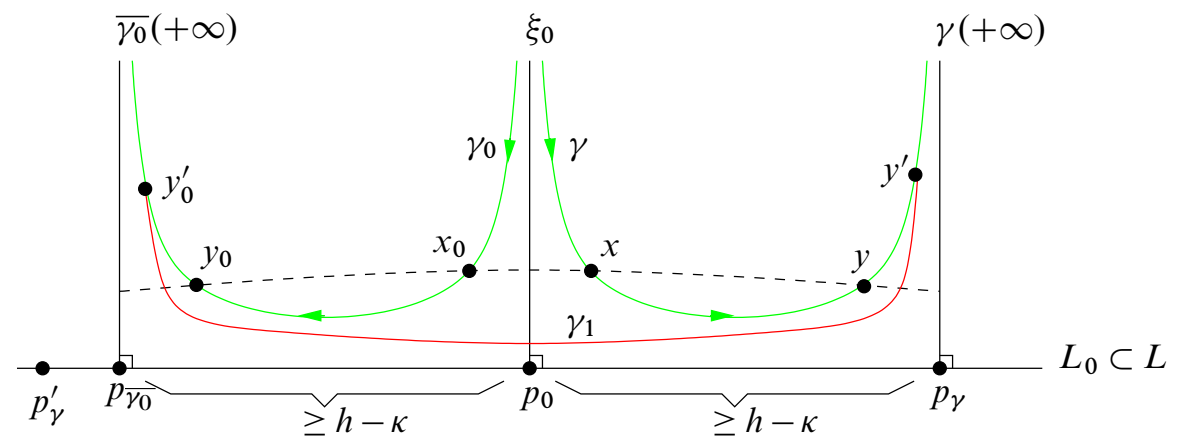

Assume by contradiction that $f^{\prime}\left(\bar{\gamma}_{0}\right)>h_{0}^{\text {min }}$. We have

$$
\ell_{C^{\prime}}(\gamma) \geq f^{\prime}(\gamma)-\kappa^{\prime} \geq h^{\prime}-\kappa^{\prime}>h_{0}^{\min }-\kappa^{\prime}=\delta .
$$

Similarly, $\ell_{C^{\prime}}\left(\bar{\gamma}_{0}\right)>\delta$, and, in particular, $\bar{\gamma}_{0}$ enters $C^{\prime}$. Let $y^{\prime}$ (resp. $y_{0}^{\prime}$ ) be the point, possibly at infinity, where $\gamma$ (resp. $\bar{\gamma}_{0}$ ) exits $C^{\prime}$. By Lemma 3.8, $\bar{\gamma}_{0}$ meets $C$ before $C^{\prime}$. Let $x_{0}$ (resp. $y_{0}$ ) be the point where $\bar{\gamma}_{0}$ enters in (resp. exits) $C$. As $\operatorname{diam}\left(C \cap C^{\prime}\right) \leq \delta$, we have $\left.y_{0}^{\prime} \in\right] y_{0}, \gamma_{0}(+\infty)\left[\right.$ and $\left.y^{\prime} \in\right] y, \gamma(+\infty)[$, so that in particular $d\left(y_{0}^{\prime}, L\right)>\epsilon$ and $d\left(y^{\prime}, L\right)>\epsilon$.

Let $\gamma_{1}$ be the geodesic line through $y^{\prime}$ and $y_{0}^{\prime}$. The points at infinity of $\gamma_{1}$ do not belong to $\partial_{\infty} L_{0}$, so that $\mathfrak{f t p}_{L_{0}}\left(\gamma_{1}\right)$ and $\ell_{C}\left(\gamma_{1}\right)$ are finite. Note that by strict convexity and by Lemma 2.3 , we have

$$
d\left(y^{\prime},\left[p_{\gamma}, \gamma(+\infty)[)<d\left(y,\left[p_{\gamma}, \gamma(+\infty)[) \leq c_{1}^{\prime}(\epsilon),\right.\right.\right.\right.
$$

and, similarly, $d\left(y_{0}^{\prime},\left[p_{\bar{\gamma}_{0}}, \bar{\gamma}_{0}(+\infty)[)<c_{1}^{\prime}(\epsilon)\right.\right.$. Hence, with $\pi_{L_{0}}$ the closest point map to $L_{0}$, which preserves betweenness and does not increase distances,

$$
\begin{aligned}
\mathfrak{f t p}_{L_{0}}\left(\gamma_{1}\right) & \geq d\left(\pi_{L_{0}}\left(y_{0}^{\prime}\right), \pi_{L_{0}}\left(y^{\prime}\right)\right) \\
& >d\left(p_{\bar{\gamma}_{0}}, p_{\gamma}\right)-2 c_{1}^{\prime}(\epsilon)=d\left(p_{\bar{\gamma}_{0}}, p_{0}\right)+d\left(p_{0}, p_{\gamma}\right)-2 c_{1}^{\prime}(\epsilon) \\
& \geq h-\kappa+h-\kappa-2 c_{1}^{\prime}(\epsilon)=2 h-2 \kappa-2 c_{1}^{\prime}(\epsilon) .
\end{aligned}
$$

In particular, by Lemma 3.4,

$$
\ell_{C}\left(\gamma_{1}\right) \geq \ell_{\mathcal{N}_{\epsilon} L_{0}}\left(\gamma_{1}\right) \geq \mathfrak{f t p}_{L_{0}}\left(\gamma_{1}\right)-2 c_{1}^{\prime}(\epsilon)-2 \epsilon>2 h-2 \kappa-4 c_{1}^{\prime}(\epsilon)-2 \epsilon \geq \delta,
$$

by the definition of $h^{\mathrm{min}}$. Hence $\gamma_{1}$ meets $C$ in a segment $I$ of length $>\delta$. But as $y_{0}^{\prime}$ and $y^{\prime}$ are at a distance strictly bigger than $\epsilon$ of $L$, the segment $I$ is contained in $\left[y^{\prime}, y_{0}^{\prime}\right]$, which is contained in $C^{\prime}$, by convexity. This contradicts the assumption that $\operatorname{diam}\left(C \cap C^{\prime}\right) \leq \delta$. 
Case (4) Let $\kappa^{\prime \prime}=\left\|f^{\prime}-\mathfrak{f t p}_{L^{\prime}}\right\|_{\infty}$. Note that $f^{\prime}(\gamma) \geq h^{\prime} \geq h_{0}^{\min }>\delta+\kappa^{\prime}$. This is true under both assumptions on the value of $h_{0}^{\min }$, as when $h_{0}^{\min }=3 c_{1}^{\prime}(\epsilon)+3 \epsilon+\delta+\kappa^{\prime \prime}$, we have, by Lemma 3.4,

$$
\delta+\kappa^{\prime} \leq \delta+\kappa^{\prime \prime}+2 c_{1}^{\prime}(\epsilon)+2 \epsilon<h_{0}^{\min } .
$$

By Lemma 3.8, we only have to prove the second claim that there exists a geodesic line $\bar{\gamma}_{0}$ starting from $\xi_{0}$ with $\bar{\gamma}_{0}(+\infty)$ belonging to $A_{0}$, such that $f^{\prime}\left(\bar{\gamma}_{0}\right) \leq h_{0}^{\min }$.

We first consider the case $C^{\prime}=\mathcal{N}_{\epsilon} L^{\prime}$ where $L^{\prime}$ is a totally geodesic subspace of codimension at least 2, with $\operatorname{diam}\left(C \cap C^{\prime}\right) \leq \delta$, and $h^{\prime} \geq h_{0}^{\min }=3 c_{1}^{\prime}(\epsilon)+3 \epsilon+\delta+\kappa^{\prime \prime}$. Assume by contradiction that every geodesic ray or line $\alpha$ starting from $\xi_{0}$ with $\alpha(+\infty) \in A_{0}$ satisfies $f^{\prime}(\alpha)>h_{0}^{\text {min }}$. In particular, every such $\alpha$ satisfies $\ell_{C^{\prime}}(\alpha) \geq$ $f^{\prime}(\alpha)-\kappa^{\prime} \geq h_{0}^{\min }-\kappa^{\prime}>\delta \geq 0$, hence $\alpha$ meets the interior of $C^{\prime}$. Let

$$
B^{\prime}=\left\{\beta(+\infty): \beta \in T_{\xi_{0}}^{1} X, \mathfrak{f t p}_{L^{\prime}}(\beta)>h_{0}^{\min }-\kappa^{\prime \prime}\right\} .
$$

By the contradiction hypothesis and the definition of $\kappa^{\prime \prime}$, we have $A_{0} \subset B^{\prime}$. Let $p_{0}^{\prime}$ be the closest point to $\xi_{0}$ on $L^{\prime}$. Note that $B^{\prime}$ is a (topological) open tubular neighbourhood of $\partial_{\infty} L^{\prime}$, whose fiber over a point $\xi$ in $\partial_{\infty} L^{\prime}$ is the preimage of $\left.\left.\rho_{\xi}(] h_{0}^{\min }-\kappa^{\prime \prime},+\infty\right]\right)$ by the closest point map from $\partial_{\infty} X$ to $L^{\prime} \cup \partial_{\infty} L^{\prime}$, where $\rho_{\xi}$ is the geodesic ray with $\rho(0)=p_{0}^{\prime}$ and $\rho(+\infty)=\xi$.

By Lemma 3.9 (a), let $\xi_{1}$ be the point at infinity of $L$ separated from $\gamma(-\infty)$ by $A_{0}$. Let $p_{\gamma(-\infty)}^{\prime}$ be the closest point to $\gamma(-\infty)$ on $L^{\prime}$. Recall that $\gamma$ enters $C^{\prime}$ at $x^{\prime}$, and $\xi_{0} \notin C^{\prime}$, so that $\xi_{0} \in\left[\gamma(-\infty), x^{\prime}[\right.$. Hence, by Lemma 2.3 and the fact that closest point maps preserve betweenness and do not increase the distances, we have $d\left(p_{\gamma(-\infty)}^{\prime}, p_{0}^{\prime}\right) \leq c_{1}^{\prime}(\epsilon)<h_{0}^{\min }-\kappa^{\prime \prime}$ by the definition of $h_{0}^{\min }$. Hence the complement of $B^{\prime}$ in $\partial_{\infty} X$, which is connected as codim $L^{\prime} \geq 2$, contains $\gamma(-\infty)$. As $A_{0}$ separates $\gamma(-\infty)$ from $\xi_{1}$ and is contained in $B^{\prime}$, it follows that $B^{\prime}$ contains $\xi_{1}$.

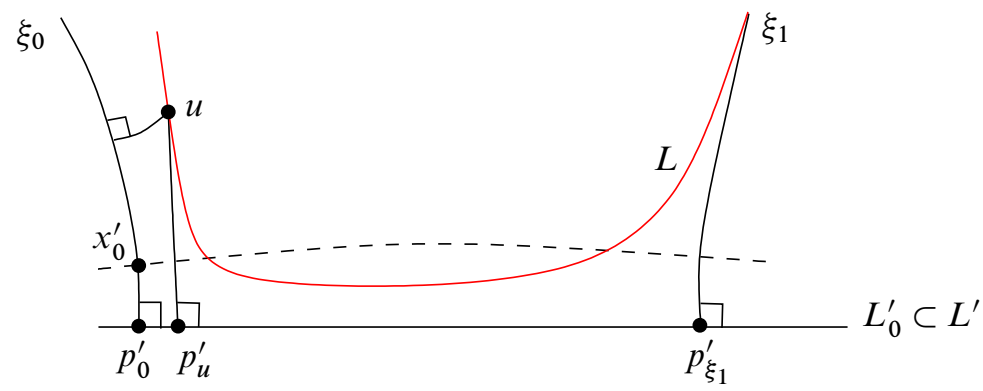

Let $x_{0}^{\prime}$ be the intersection point of $\left.] \xi_{0}, p_{0}^{\prime}\right]$ with $\partial C^{\prime}$. Lemma 2.3 then implies that $d\left(x^{\prime}, x_{0}^{\prime}\right) \leq c_{1}^{\prime}(\epsilon)$. Hence, by convexity and as $\gamma$ first meets $C$ and then $C^{\prime}$, we have 
$\left.\left.d(x,] \xi_{0}, p_{0}^{\prime}\right]\right) \leq c_{1}^{\prime}(\epsilon)$. If $u \in L$ is the closest point to $x$ on $L$, we therefore have $\left.\left.d(u,] \xi_{0}, p_{0}^{\prime}\right]\right) \leq c_{1}^{\prime}(\epsilon)+\epsilon$. Let $p_{\xi_{1}}^{\prime}$ be the closest point to $\xi_{1}$ on $L^{\prime}$, and $L_{0}^{\prime}$ the geodesic line (contained in $L^{\prime}$ ) through $p_{0}^{\prime}$ and $p_{\xi_{1}}^{\prime}$. As the closest point map does not increase distances, the closest point $p_{u}^{\prime}$ to $u$ on $L_{0}^{\prime}$ satisfies $d\left(p_{0}^{\prime}, p_{u}^{\prime}\right) \leq c_{1}^{\prime}(\epsilon)+\epsilon$. Then, since the closest point map to $L_{0}^{\prime}$ preserves betweenness, by the triangle inequality, and as $\xi_{1}$ belongs to $B^{\prime}$, we have

$$
\mathfrak{f t p}_{L_{0}^{\prime}}(L) \geq d\left(p_{u}^{\prime}, p_{\xi_{1}}^{\prime}\right) \geq d\left(p_{\xi_{1}}^{\prime}, p_{0}^{\prime}\right)-d\left(p_{0}^{\prime}, p_{u}^{\prime}\right)>h_{0}^{\min }-\kappa^{\prime \prime}-c_{1}^{\prime}(\epsilon)-\epsilon .
$$

Therefore, using Lemma 3.4,

$$
\begin{aligned}
\operatorname{diam}\left(C \cap C^{\prime}\right) & \geq \operatorname{diam}\left(C \cap \mathcal{N}_{\epsilon} L_{0}^{\prime}\right) \geq \ell_{\mathcal{N}_{\epsilon} L_{0}^{\prime}}(L) \geq \mathfrak{f t p}_{L_{0}^{\prime}}(L)-2 c_{1}^{\prime}(\epsilon)-2 \epsilon \\
& >h_{0}^{\min }-\kappa^{\prime \prime}-3 c_{1}^{\prime}(\epsilon)-3 \epsilon=\delta
\end{aligned}
$$

a contradiction.

Assume now that $C^{\prime}$ is any $\epsilon$-convex subset such that $C \cap C^{\prime}=\varnothing$, and that $h^{\prime}>$ $h_{0}^{\min }=\kappa^{\prime \prime}$. Let us prove that there exists a geodesic ray or line $\bar{\gamma}_{0}$ starting from $\xi_{0}$ with $\bar{\gamma}_{0}(+\infty)$ in $A_{0}$, and avoiding the interior of $C^{\prime}$. This implies the result as in Case (1).

By contradiction, suppose that for every $\xi$ in $A_{0}$, the geodesic ray or line $\gamma_{\xi}$ starting from $\xi_{0}$ and ending at $\xi$ meets the interior of $C^{\prime}$. By Lemma 3.8 (applied with $\delta=0$ ), $\gamma_{\xi}$ meets the interior of $C$ before meeting $C^{\prime}$. Let $x_{\xi}^{\prime}$ be the entering point of $\gamma_{\xi}$ in $C^{\prime}$ and $y_{\xi}$ be its exiting point out of $C$. As $C$ and $C^{\prime}$ are disjoint, note that $\xi_{0}, y_{\xi}, x_{\xi}^{\prime}, \xi$ are in this order along $\gamma_{\xi}$. The maps $\xi \mapsto y_{\xi}$ and $\xi \mapsto x_{\xi}^{\prime}$ are injective and continuous on $A_{0}$ (by the strict convexity of $C$ and $C^{\prime}$, as $\gamma_{\xi}$ meets the interior of $C$ and $C^{\prime}$ ). We know that $A_{0}$ is a topological sphere, by Lemma 3.9 (a), separating the endpoints of $L$. Hence the subsets $A_{0}$ and $S^{\prime}=\left\{x_{\xi}^{\prime}: \xi \in A_{0}\right\}$ are spheres, that are homotopic (by the homotopy along the geodesic ray or line $\gamma_{\xi}$ that does not meet $L$ between $x_{\xi}^{\prime}$ and $\xi$ ) in the complement of $L$ in $X \cup \partial_{\infty} X$. By a homology argument, every disc with boundary $S^{\prime}$ in $X \cup \partial_{\infty} X$ has to meet $L$. But by convexity of $C^{\prime}$, there exists a disc contained in $C^{\prime}$ with boundary $S^{\prime}$ (fix a point of $S^{\prime}$ and take the union of the geodesic arcs from this point to the other points of $S^{\prime}$ ). This contradicts the fact that $C \cap C^{\prime}=\varnothing$.

Remarks (1) In Case (2), we have $h^{\max } \geq h^{\min }$ if $R$ is big enough, as $c_{1}^{\prime}(\epsilon)$ has a finite limit as $\epsilon \rightarrow \infty$.

(2) In Case (3), if the codimension of $L$ is 1 , then we may assume that $\bar{\gamma}$ meets $L$ if $\gamma$ meets $L$. Indeed, as we have seen in Lemma 3.9 (b), $L \cup \partial_{\infty} L$ separates $X \cup \partial_{\infty} X$ into two connected components, and $A$ (defined in the beginning of the proof) has 
exactly two components separated by $L \cup \partial_{\infty} L$. If $A_{0}^{+}$is the component of $A$ on the same side of $L \cup \partial_{\infty} L$ as $\xi_{0}$, and $A_{0}^{-}$the component of $A$ on the other side, then a geodesic ray or line starting from $\xi_{0}$ and ending in $A_{0}^{+}$does not meet $L$ (as $L$ is totally geodesic), and any geodesic line starting from $\xi_{0}$ and ending in $A_{0}^{-}$meets $L$, by separation. This observation on the crossing property will be used in the proof of Corollary 5.12 to make sure that the locally geodesic ray or line constructed in the course of the proof stays in the convex core.

(3) Case (4) is not true if $C^{\prime}$ is assumed to be any $\epsilon$-convex subset, as shown by taking $X$ the real hyperbolic 3-space, and $C^{\prime}$ the $\epsilon$-neighbourhood of the (totally geodesic) hyperbolic plane perpendicular to $L$ at a point at distance $h$ from the closest point to $\xi_{0}$ on $L$ : any geodesic ray or line $\alpha$ starting from $\xi_{0}$, with $\mathfrak{f t p}_{L}(\alpha)=h$ and meeting $C^{\prime}$ satisfies $f^{\prime}(\alpha)=+\infty$ for every $f^{\prime}$ which is a $\kappa^{\prime}$-penetration map in $C^{\prime}$.

\section{The main construction}

\subsection{Unclouding the sky}

The aim of this section is to prove the following result, improving on our result in [47]. The first claim of Theorem 4.1 was stated as Theorem 1.1 in the introduction.

Theorem 4.1 Let $X$ be a proper geodesic CAT(-1) metric space (having at least two points), with arcwise connected boundary $\partial_{\infty} X$ and extendible geodesics. Let $\left(H_{\alpha}\right)_{\alpha \in \mathcal{A}}$ be any family of balls or horoballs with pairwise disjoint interiors. Let $\mu_{0}=1.534$.

(1) For every $x$ in $X-\bigcup_{\alpha \in \mathcal{A}} H_{\alpha}$, there exists a geodesic ray starting from $x$ and avoiding $H_{\alpha}\left[\mu_{0}\right]$ for every $\alpha$.

(2) For every $\alpha_{0}$ in $\mathcal{A}$ such that $H_{\alpha_{0}}$ is an horoball, there exists a geodesic line starting from the point at infinity of $H_{\alpha_{0}}$ and avoiding $H_{\alpha}\left[\mu_{0}\right]$ for every $\alpha \neq \alpha_{0}$.

Remarks (1) Note that by its generality, Theorem 4.1 greatly improves the main results, Theorem 1.1 and Theorem 4.5, of [47], where (except for trees) $X$ was always assumed to be a manifold, strict assumptions were made on the boundary of $X$, and no definite value of $\mu_{0}$ was given except in special cases. But besides this, an important point is that its proof is a much simplified version of the upcoming main construction of Section 4, and hence could be welcome as a guide for reading Section 4.2.

(2) Note that the constant $\mu_{0}$ is not optimal, but not by much. For simplicial trees all of whose vertices have degree at least 3 , the result is true, with any $\mu_{0}>1$ and 
this is optimal (though they do not satisfy the hypotheses of the above result, the proof is easy for them; see for instance [47, Theorem 7.2 (3)]). We proved in [47] that the optimal value for the second assertion of the theorem, when $X=\mathbb{H}_{\mathbb{R}}^{n}$ and all $H_{\alpha}$ 's are horoballs, is $\mu_{0}=-\log (4 \sqrt{2}-5) \approx 0.42$. Hence Theorem 4.1 (2) is not far from optimal, despite its generality. Furthermore, when $X=\mathbb{H}_{\mathbb{R}}^{n}$ and all $H_{\alpha}$ 's are horoballs, a possible value of $\mu_{0}$ for the first assertion of the theorem that was given in [47, Theorem 7.1] was $\log (2+\sqrt{5})-\log (4 \sqrt{2}-5) \approx 1.864$. Hence Theorem 4.1 (1) is even better than the corresponding result in [47] when $X=\mathbb{H}_{\mathbb{R}}^{n}$, despite its generality.

Proof We start with the following geometric lemma. For every $\mu \geq 0$, define

$$
v(\mu)=\frac{2 e^{-\mu}}{1+\sqrt{1-e^{-2 \mu}}},
$$

which is positive and decreasing from 2 to 0 as $\mu$ goes from 0 to $+\infty$.

Lemma 4.2 Let $X$ be a proper geodesic CAT(-1) space. Let $H$ be a ball or a horoball in $X$ and $\xi_{0} \in\left(X \cup \partial_{\infty} X\right)-(H \cup H[\infty])$. Let $\mu \geq \log 2$ be at most the radius of $H$, and let $\gamma$ and $\gamma^{\prime}$ be geodesic rays or lines starting at $\xi_{0}$, meeting $H[\mu]$, parametrized such that $\gamma^{\prime}(s), \gamma(s)$ are equidistant to $\xi_{0}$ for some (hence every) $s$, and that $\gamma$ enters $H$ at time 0 .

(1) If $x=\gamma(0)$ and $x^{\prime}$ are the points of entry in $H$ of $\gamma$ and $\gamma^{\prime}$ respectively, then $d\left(x, x^{\prime}\right) \leq v(\mu)$.

(2) For every $s \geq 0$, we have

$$
d\left(\gamma(-s), \gamma^{\prime}(-s)\right) \leq v(\mu) e^{-s} .
$$

Proof Let $\xi$ be the center or point at infinity of $H$, and let $t, t^{\prime}$ be the entrance times of $\gamma, \gamma^{\prime}$ respectively in $H[\mu]$. Note that $t \geq 0$ as $\mu \geq 0$. Let us prove first that $t^{\prime} \geq 0$ too. We refer to Section 2.1 for the definition and properties of the map $\beta_{\xi_{0}}$, especially when $\xi_{0} \in X$. Let $u$ be the point on the geodesic ] $\xi_{0}, \xi$ [ such that $\beta_{\xi_{0}}(x, u)=0$. By the convexity of the balls and horoballs, the point $u$ is the closest point to $\xi$ on the sphere or horosphere centered at $\xi_{0}$ passing through $x$. Since $\beta_{\xi_{0}}\left(x, \gamma^{\prime}(0)\right)=0$, we hence have $\beta_{\xi}\left(x, \gamma^{\prime}(0)\right) \leq \beta_{\xi}(x, u)$. Let us prove that $\beta_{\xi}(x, u) \leq \mu$, which will hence imply that $\gamma^{\prime}$ enters $H[\mu]$ at a nonnegative time (which is $t^{\prime}$ ).

Glue the two comparison triangles $\left(\overline{\xi_{0}}, \bar{x}, \bar{\xi}\right)$ and $(\bar{\xi}, \bar{x}, \overline{\gamma(t)})$ in $\mathbb{H}_{\mathbb{R}}^{2}$ for the geodesic triangles $\left(\xi_{0}, x, \xi\right)$ and $(\xi, x, \gamma(t))$ along their sides $[\bar{x}, \bar{\xi}]$. 


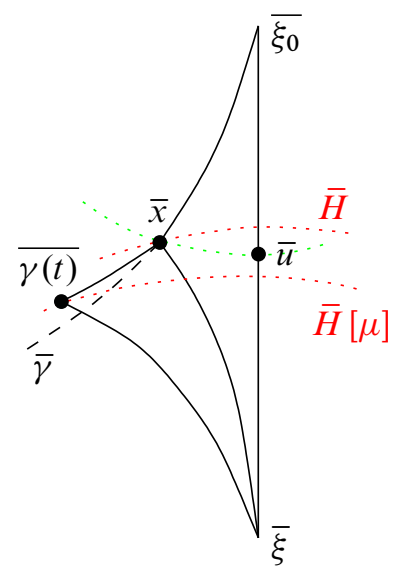

Let $\bar{H}$ be the ball or horoball centered at $\bar{\xi}$ such that $\bar{x} \in \partial \bar{H}$. By comparison, we have $\angle_{\bar{x}}\left(\overline{\xi_{0}}, \bar{\xi}\right) \leq \pi \leq \angle_{\bar{x}}\left(\overline{\xi_{0}}, \bar{\xi}\right)+\angle_{\bar{x}}(\bar{\xi}, \overline{\gamma(t)})$. Hence the geodesic ray or line $\bar{\gamma}$ starting from $\xi_{0}$ and passing through $\bar{x}$ meets $[\overline{\gamma(t)}, \bar{\xi}]$, therefore it enters $\bar{H}[\mu]$. Let $\bar{u}$ be the point on the geodesic $\left[\overline{\xi_{0}}, \bar{\xi}\right]$ such that $\beta_{\overline{\xi_{0}}}(\bar{x}, \bar{u})=0$. As $\beta_{\xi}(x, u)=\beta_{\bar{\xi}}(\bar{x}, \bar{u})$, we only have to prove the result if $X$ is the upper halfspace model of the hyperbolic plane $\mathbb{H}_{\mathbb{R}}^{2}$.

We may then assume that $\xi_{0}$ is the point at infinity $\infty$, and that $H$ is the horoball with point at infinity 0 and Euclidean diameter 1 (see the figure below). But then, the vertical coordinate of $\gamma(0)$ is at least $\frac{1}{2}$, since $\gamma$ enters $H$ at $\gamma(0)$. As $e^{-\mu} \leq \frac{1}{2}$, the result follows: any geodesic line, starting from $\xi_{0}$ and meeting $H[\mu]$, meets the horizontal horosphere containing $\gamma(0)$ before $H[\mu]$.

Now, in order to prove both assertions of Lemma 4.2, let us show that we may assume that $X=\mathbb{H}_{\mathbb{R}}^{2}$.

For the first one, glue the two comparison triangles $\left(\overline{\xi_{0}}, \bar{x}, \bar{\xi}\right)$ and $\left(\overline{\xi_{0}}, \overline{x^{\prime}}, \bar{\xi}\right)$ for the geodesic triangles $\left(\xi_{0}, x, \xi\right)$ and $\left(\xi_{0}, x^{\prime}, \xi\right)$ along their sides $\left[\overline{\xi_{0}}, \bar{\xi}\right]$. As seen above, the geodesic line $\bar{\gamma}$ (resp. $\overline{\gamma^{\prime}}$ ) starting from $\overline{\xi_{0}}$ and passing through $\bar{x}$ (resp. $\overline{x^{\prime}}$ ) enters $\bar{H}[\mu]$. And by comparison, we have $d\left(x, x^{\prime}\right) \leq d\left(\bar{x}, \overline{x^{\prime}}\right)$.

For the second assertion, we glue the two comparison triangles $\left(\overline{\xi_{0}}, \overline{\gamma(t)}, \overline{\gamma^{\prime}\left(t^{\prime}\right)}\right)$ and $\left(\bar{\xi}, \overline{\gamma(t)}, \overline{\gamma^{\prime}\left(t^{\prime}\right)}\right)$ for the geodesic triangles $\left(\xi_{0}, \gamma(t), \gamma^{\prime}\left(t^{\prime}\right)\right)$ and $\left(\xi, \gamma(t), \gamma^{\prime}\left(t^{\prime}\right)\right)$ along their isometric segments $\left[\overline{\gamma(t)}, \overline{\gamma^{\prime}\left(t^{\prime}\right)}\right]$. As in the beginning of the proof of Lemma 2.3, the geodesic segment or ray $] \overline{\xi_{0}}, \overline{\gamma(t)}$ [ does not meet the ball or horoball $\overline{H[\mu]}$ centered at $\bar{\xi}$ whose boundary goes through $\overline{\gamma(t)}$ and $\overline{\gamma^{\prime}\left(t^{\prime}\right)}$. By comparison, if $H^{\prime}$ is the ball or horoball centered at $\bar{\xi}$ whose boundary passes through the point $\overline{\gamma(0)}$ on $] \overline{\xi_{0}}, \overline{\gamma(t)}\left[\right.$ at distance $t$ from $\overline{\gamma(t)}$, then $H^{\prime}[\mu]$ contains $\overline{H[\mu]}$, so that $\left.] \overline{\xi_{0}}, \overline{\gamma(t)}\right]$ 
and ] $\left.\overline{\xi_{0}}, \overline{\gamma^{\prime}\left(t^{\prime}\right)}\right]$ meet $H^{\prime}[\mu]$. For every $s \geq 0$, as $t, t^{\prime} \geq 0$, if $\overline{\gamma(-s)}, \overline{\gamma^{\prime}(-s)}$ are the corresponding points to $\gamma(-s), \gamma^{\prime}(-s)$ on $] \overline{\xi_{0}}, \overline{\gamma(t)}[,] \overline{\xi_{0}}, \overline{\gamma^{\prime}\left(t^{\prime}\right)}$ [ respectively, then by comparison $d\left(\gamma(-s), \gamma^{\prime}(-s)\right) \leq d\left(\overline{\gamma(-s)}, \overline{\gamma^{\prime}(-s)}\right)$.

Hence we may assume that $X$ is the upper halfspace model of the real hyperbolic plane $\mathbb{H}_{\mathbb{R}}^{2}$. Up to replacing $\xi_{0}$ by the point at infinity $\xi_{0}^{\prime}$ of a geodesic ray starting perpendicularly from the boundary of $H$ and passing through $\xi_{0}$, and $\gamma, \gamma^{\prime}$ by the geodesic lines starting from $\xi_{0}^{\prime}$ and passing through $x, x^{\prime}$, we may assume that $\xi_{0}$ is at infinity.

By homogeneity and monotonicity, it is sufficient to prove the result for $\xi_{0}$ the point at infinity $\infty$, for $H$ the horoball with point at infinity 0 and Euclidean diameter 1, and with $\gamma$ and $\gamma^{\prime}$ different and both tangent to $H[\mu]$.

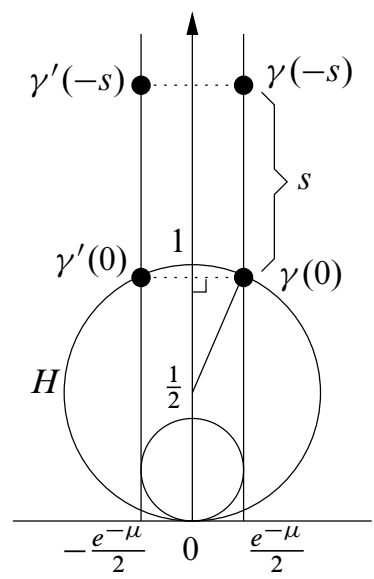

Then, by an easy computation, the Euclidean height of the point $\gamma(0)$ is $\nu^{\prime}(\mu)=$ $\frac{1}{2}\left(1+\sqrt{1-e^{-2 \mu}}\right)$, so that the Euclidean height of the point $\gamma(-s)$ is $v^{\prime}(\mu) e^{s}$. The hyperbolic distance between $\gamma(-s)$ and $\gamma^{\prime}(-s)$ is hence at most $e^{-\mu} /\left(v^{\prime}(\mu) e^{s}\right)=$ $v(\mu) e^{-s}$. With the case $s=0$, this proves both assertions.

Proof of Theorem 4.1 Let $X$ and $\left(H_{\alpha}\right)_{\alpha \in \mathcal{A}}$ be as in the statement. Let $\xi_{0}$ be either a point in $X-\bigcup_{\alpha \in \mathcal{A}} H_{\alpha}$ or the point at infinity of $H_{\alpha_{0}}$ for some $\alpha_{0}$ in $\mathcal{A}$ such that $H_{\alpha_{0}}$ is a horoball. For every $\mu_{1} \geq \log 2$, define the following constants, with $v$ the map introduced before Lemma 4.2,

$$
\mu_{2}=v\left(\mu_{1}\right)>0, \quad \mu_{3}=\mu_{1}+\mu_{2}>0, \quad \mu_{4}=2 \mu_{1}-2 \mu_{2} .
$$

As $\mu_{1} \geq \log 2, v$ is decreasing and $v(\log 2)<\log 2$, we have $\mu_{4}>0$. We define by induction an initial segment $\mathcal{N}$ in $\mathbb{N}$ and the following finite or infinite sequences 
- $\left(\gamma_{k}\right)_{k \in \mathcal{N}}$ of geodesic rays or lines starting from $\xi_{0}$,

- $\left(\alpha_{k}\right)_{k \in \mathcal{N}-\{0\}}$ of elements in $\mathcal{A}$,

- $\left(t_{k}\right)_{k \in \mathcal{N}}$ of nonnegative real numbers,

- $\left(u_{k}\right)_{k \in \mathcal{N}}$ of maps $u_{k}:[0,+\infty[\rightarrow] 0,+\infty[$,

such that for every $k$ in $\mathcal{N}$, the following assertions hold:

(1) If $\xi_{0} \in X$, then $\gamma_{k}(0)=\xi_{0}$. Otherwise, $\gamma_{k}$ meets $\partial H_{\alpha_{0}}$ at time 0 .

(2) If $k \geq 1$, then $\gamma_{k}$ enters $H_{\alpha_{k}}$ at the point $\gamma_{k}\left(t_{k}\right)$ and meets $H_{\alpha_{k}}\left[\mu_{1}\right]$ in one and only one point.

(3) If $k \geq 1$, then $u_{k}(t)=u_{k-1}(t)+\mu_{2} e^{t-t_{k}}$ if $t \leq t_{k-1}$, and $u_{k}(t)=\mu_{3}$ if $t>t_{k-1}$.

(4) If $k \geq 1$, then $t_{k} \geq \mu_{4}+t_{k-1}$.

(5) If $t \in\left[0, t_{k}\left[\right.\right.$, then the point $\gamma_{k}(t)$ does not belong to $\bigcup_{\alpha \in \mathcal{A}} H_{\alpha}\left[u_{k}(t)\right]$.

If $\xi_{0} \in X$, let $\gamma_{0}$ be a geodesic ray starting from $\xi_{0}$ at time 0 . Otherwise, let $\gamma_{0}$ be a geodesic line starting from $\xi_{0}$ and exiting $H_{\alpha_{0}}$ at time 0 . Such a $\gamma_{0}$ exists by the assumptions on $X$. Define $u_{0}$ as the constant map $t \mapsto \mu_{3}$. Let $t_{0}=0$. The assertions (1)-(5) are satisfied for $k=0$. Assume that $\gamma_{k}, t_{k}, \alpha_{k}, u_{k}$ are constructed for $0 \leq k \leq n$ verifying the assertions (1)-(5).

If the geodesic ray $\gamma_{n}(] t_{n},+\infty[)$ does not enter in the interior of any element of the family $\left(H_{\alpha}\left[\mu_{1}\right]\right)_{\alpha \in \mathcal{A}}$, then define $\mathcal{N}=[0, n] \cap \mathbb{N}$, and the construction terminates. Otherwise, let $H_{\alpha_{n+1}}\left[\mu_{1}\right]$ be the first element of the family $\left(H_{\alpha}\left[\mu_{1}\right]\right)_{\alpha \in \mathcal{A}}$ such that the geodesic ray $\gamma_{n}(] t_{n},+\infty[)$ enters in its interior. Such an element exists as the $H_{\alpha}$ 's have disjoint interiors. Note that $\alpha_{n+1} \neq \alpha_{n}$, as $\gamma_{n}$ does not meet the interior of $H_{\alpha_{n}}\left[\mu_{1}\right]$ by (2).

If $\xi_{0} \in X$, let $\gamma_{n+1}$ be a geodesic ray starting from $\xi_{0}$ at time 0 and meeting $H_{\alpha_{n+1}}\left[\mu_{1}\right]$ in one and only one point. This is possible as there exists a geodesic ray starting from $\xi_{0}$ and avoiding $H_{\alpha_{n+1}}$ by the properties of $X$ (consider for instance the extension to ] $-\infty, 0]$ of $\gamma_{n}$ ) and since $\partial_{\infty} X$ is arcwise connected. If $\xi_{0} \notin X$, let $\gamma_{n+1}$ be a geodesic line starting from $\xi_{0}$, and meeting $H_{\alpha_{n+1}}\left[\mu_{1}\right]$ in one and only one point. Again, this is possible as $\partial_{\infty} X$ is arcwise connected. Parametrize $\gamma_{n+1}$ such that $\gamma_{n+1}$ exits $H_{\alpha_{0}}$ at time 0 . In particular, in both cases, the assertion (1) for $k=n+1$ is satisfied.

Define $t_{n+1} \geq 0$ such that $\gamma_{n+1}$ enters $H_{\alpha_{n+1}}$ at the point $\gamma_{n+1}\left(t_{n+1}\right)$, so that the assertion (2) for $k=n+1$ is satisfied. As $\gamma_{n}$ and $\gamma_{n+1}$ both meet $H_{\alpha_{n+1}}\left[\mu_{1}\right]$ and as $\mu_{1} \geq \log 2$, it follows from Lemma 4.2 (2) that, for every $t \leq t_{n+1}$,

$$
d\left(\gamma_{n+1}(t), \gamma_{n}(t)\right) \leq \mu_{2} e^{t-t_{n+1}} .
$$


Define $\tau_{n} \geq t_{n}$ as the entrance time of $\gamma_{n}$ in $H_{\alpha_{n+1}}$. By Lemma 4.2 (1), as both $\gamma_{n}$ and $\gamma_{n+1}$ meet $H_{\alpha_{n+1}}\left[\mu_{1}\right]$ and $\mu_{1} \geq \log 2$, we have

$$
d\left(\gamma_{n+1}\left(t_{n+1}\right), \gamma_{n}\left(\tau_{n}\right)\right) \leq \mu_{2} .
$$

As $H_{\alpha_{n+1}}$ and $H_{\alpha_{n}}$ have disjoint interiors, and since $H_{\alpha_{n}}$ and $H_{\alpha_{n}}\left[\mu_{1}\right]$ are at distance $\mu_{1}$, we have $d\left(\gamma_{n}\left(t_{n}\right), \gamma_{n}\left(\tau_{n}\right)\right) \geq 2 \mu_{1}$. Hence

$$
\begin{aligned}
d\left(\gamma_{n}\left(t_{n}\right), \gamma_{n}\left(t_{n+1}\right)\right) & \\
& \geq d\left(\gamma_{n}\left(t_{n}\right), \gamma_{n}\left(\tau_{n}\right)\right)-d\left(\gamma_{n}\left(\tau_{n}\right), \gamma_{n+1}\left(t_{n+1}\right)\right)-d\left(\gamma_{n+1}\left(t_{n+1}\right), \gamma_{n}\left(t_{n+1}\right)\right) \\
& \geq 2 \mu_{1}-2 \mu_{2}=\mu_{4}>0 .
\end{aligned}
$$

In particular $t_{n+1}-t_{n}$ is positive (otherwise $\gamma_{n}\left(t_{n}\right)$ belongs to $\left[\gamma_{n}\left(t_{n+1}\right), \gamma_{n}\left(\tau_{n}\right)\right]$, hence

$$
\begin{aligned}
2 \mu_{1} \leq d\left(\gamma_{n}\left(t_{n}\right), \gamma_{n}\left(\tau_{n}\right)\right) & \leq d\left(\gamma_{n}\left(t_{n+1}\right), \gamma_{n}\left(\tau_{n}\right)\right) \\
& \leq d\left(\gamma_{n}\left(\tau_{n}\right), \gamma_{n+1}\left(t_{n+1}\right)\right)+d\left(\gamma_{n+1}\left(t_{n+1}\right), \gamma_{n}\left(t_{n+1}\right)\right) \leq 2 \mu_{2},
\end{aligned}
$$

a contradiction). Therefore $t_{n+1}-t_{n}$ is at least $\mu_{4}$, which proves the assertion (4) for $k=n+1$.

Define $t \mapsto u_{n+1}(t)$ by the induction formula in Assertion (3). The only remaining assertion to verify is (5). By contradiction, assume that there exist some $t$ in $\left[0, t_{n+1}[\right.$ and some $\alpha \in \mathcal{A}$ such that $\gamma_{n+1}(t)$ belongs to $H_{\alpha}\left[u_{n+1}(t)\right]$. As $u_{n+1}(t)>0$, the element $\alpha$ is different from $\alpha_{0}$ if $\xi_{0} \in \partial_{\infty} X$, and it is also different from $\alpha_{n+1}$ by construction. By Equation (15), the point $\gamma_{n}(t)$ belongs to $H_{\alpha}\left[u_{n+1}(t)-\mu_{2} e^{t-t_{n+1}}\right]$. Assume first that $t>t_{n}$, so that $u_{n+1}(t)=\mu_{3}$. As $\mu_{3}-\mu_{2} e^{t-t_{n+1}}>\mu_{1}$ (since $t<t_{n+1}$ and by the definition of $\mu_{3}$ ), this implies that $\gamma_{n}(t)$ belongs to the interior of $H_{\alpha}\left[\mu_{1}\right]$. This contradicts the fact that $H_{\alpha_{n+1}}\left[\mu_{1}\right]$ is the first element of the family $\left(H_{\alpha}\left[\mu_{1}\right]\right)_{\alpha \in \mathcal{A}}$ encountered by $\gamma_{n}(] t_{n},+\infty[)$ in its interior.

Assume now that $t \leq t_{n}$. Then $\gamma_{n}(t)$ belongs to $H_{\alpha}\left[u_{n}(t)\right]$. This contradicts the assertion (5) at step $n$. Thus, the assertions (1)-(5) hold for all $k \in \mathcal{N}$.

Let us prove that the maps $u_{n}$ are uniformly bounded from above by

$$
\mu_{5}=\mu_{3}+\frac{\mu_{2}}{e^{\mu_{4}}-1} .
$$

As $\mu_{4}>0$, the sequence $\left(t_{k}\right)_{k \in \mathbb{N}}$ increases to $+\infty$. Fix $t \geq 0$. Let $k=k(t)$ be the unique nonnegative integer such that $t$ belongs to $] t_{k-1}, t_{k}$ (by convention, $t_{-1}=-\infty$ ). Let us prove, by induction on $n$, that

$$
u_{n}(t) \leq \mu_{3}+\mu_{2} \sum_{j=1}^{n-k} e^{-\mu_{4} j}
$$


(Recall that an empty sum is 0 ). This implies that $u_{n}(t) \leq \mu_{5}$.

This is true if $n=0$, as $u_{0}(t)=\mu_{3}$. Assume that the result is true for $n$. If $t>t_{n}$, then $u_{n+1}(t)=\mu_{3}$, and the result is true. Otherwise, by property (3), we have $u_{n+1}(t)=u_{n}(t)+\mu_{2} e^{t-t_{n+1}}$. Note that $t_{k}-t_{n+1} \leq-\mu_{4}(n+1-k)$ by property (4), and that $t \leq t_{k}$. Hence, by induction,

$$
u_{n+1}(t) \leq \mu_{3}+\mu_{2} \sum_{j=1}^{n-k} e^{-\mu_{4} j}+\mu_{2} e^{-\mu_{4}(n+1-k)}=\mu_{3}+\mu_{2} \sum_{j=1}^{n+1-k} e^{-\mu_{4} j} .
$$

This proves the induction.

Summarizing the above construction, there exist a sequence of geodesic rays or lines $\left(\gamma_{n}\right)_{n \in \mathbb{N}}$ starting from $\xi_{0}$, and a sequence of times $\left(t_{n}\right)_{n \in \mathbb{N}}$ converging to $+\infty$, such that for every $t$ in $\left[0, t_{n}\right]$, the point $\gamma_{n}(t)$ does not belong to $\bigcup_{\alpha \in \mathcal{A}} H_{\alpha}\left[\mu_{5}\right]$. (Take an eventually constant sequence $\left(\gamma_{n}\right)_{n \in \mathbb{N}}$ if the construction stops at a finite stage, which is possible as $\mu_{5}>\mu_{1}$.) As $\left(t_{n}\right)_{n \in \mathbb{N}}$ grows at least linearly, the formula (15) implies that $\left(\gamma_{n}(t)\right)_{n \in \mathbb{N}}$ is a Cauchy sequence, uniformly on every compact subset of nonnegative $t$ 's. Hence, the geodesic rays or lines $\gamma_{n}$ converge to a geodesic ray or line avoiding $\bigcup_{\alpha \in \mathcal{A}} H_{\alpha}\left[\mu_{5}-\epsilon\right]$, for every $\epsilon>0$. Taking $\mu_{1}=1.042 \geq \log 2$, we can check that $\mu_{5}<1.5332$, hence the result follows.

Corollary 4.3 Let $X$ and $\left(H_{\alpha}\right)_{\alpha \in \mathcal{A}}$ be as in Theorem 4.1. For every $x \in X$, there exist $t>0$ and a geodesic ray $\gamma$ starting at $x$ such that $\gamma([t, \infty[)$ is contained in the complement of $\bigcup_{\alpha \in \mathcal{A}} H_{\alpha}\left[\mu_{0}\right]$.

Proof We may assume that $x \in H_{\alpha_{0}}$ for some $\alpha_{0} \in \mathcal{A}$, otherwise, Theorem 4.1 (1) applies (with $t=0$ ). Let $H_{\alpha}^{\prime}=H_{\alpha}$ if $\alpha \neq \alpha_{0}$, and $H_{\alpha_{0}}^{\prime}=H_{\alpha_{0}}\left[d\left(x, \partial H_{\alpha_{0}}\right)+1\right]$. Then $x \notin X-\bigcup_{\alpha \in \mathcal{A}} H_{\alpha}^{\prime}$. By Theorem 4.1 (1), let $\gamma$ be a geodesic ray starting from $x$ and avoiding the $H_{\alpha}^{\prime}\left[\mu_{0}\right]$ 's. Let $t=d\left(x, \partial H_{\alpha_{0}}\right)+2+2 \mu_{0}+2 \log (1+\sqrt{2})$. Recall that $\left\|\ell_{H_{\alpha_{0}}}-\mathfrak{p h}_{H_{\alpha_{0}}}\right\|_{\infty} \leq 2 \log (1+\sqrt{2})$ by Section 3.1. With $\bar{\gamma}$ any extension of $\gamma$ to a full geodesic line, the length of the geodesic segment $\gamma \cap H_{\alpha_{0}}$ is at most

$$
\ell_{H_{\alpha_{0}}}(\bar{\gamma})-d\left(x, \partial H_{\alpha_{0}}\right) \leq \mathfrak{p h}_{H_{\alpha_{0}}}(\bar{\gamma})+2 \log (1+\sqrt{2})-d\left(x, \partial H_{\alpha_{0}}\right)<t,
$$

since $\gamma$ avoids $H_{\alpha_{0}}\left[d\left(x, \partial H_{\alpha_{0}}\right)+1+\mu_{0}\right]$. Hence the geodesic ray $\gamma([t, \infty[)$ does not meet $H_{\alpha_{0}}$. The result follows.

Let $e$ be an end of a finite volume complete negatively curved Riemannian manifold $V$. Let ht $e$ be the Busemann function of $e$ normalized to be zero on the boundary of the maximal Margulis neighbourhood of $e$ (see for instance Buser and Karcher [12], 
Hersonsky and Paulin [30] and Parkkonen and Paulin [47], as well as the paragraph above Corollary 5.4). Our next result improves Theorem 7.4 (hence Corollary 1.2) in [47], with the same proof, by removing the technical assumptions on the manifold and giving a universal upper bound on $h_{e}(V)$.

Corollary 4.4 Let $V$ be a finite volume complete Riemannian manifold with dimension at least 2 and sectional curvature $K \leq-1$, and let $e$ be an end of $V$. Then there exists a closed geodesic in $V$ whose maximum height (with respect to ht $t_{e}$ ) is at most 1.534 .

\subsection{The inductive construction}

Fix arbitrary constants $\epsilon_{0} \in \mathbb{R}_{+}^{*} \cup\{\infty\}$ and $\delta_{0}, \kappa_{0} \geq 0$, and fix an arbitrary point $\xi_{0}$ in $X \cup \partial_{\infty} X$. Let $\left(C_{n}\right)_{n \in \mathbb{N}}$ be a family of $\epsilon_{0}$-convex subsets of $X$ such that $\xi_{0} \notin C_{0} \cup \partial_{\infty} C_{0}$, and let $f_{0}$ be a $\kappa_{0}$-penetration map for $C_{0}$.

The aim of this section is to construct by induction a sequence of geodesic rays or lines in $X$, starting from $\xi_{0}$ and having a suitable penetration behaviour in the $C_{n}$ 's.

Prescription of constants The following constants will appear in the statement, or in the proof, of the inductive construction:

- $c_{1}=c_{1}^{\prime}\left(\epsilon_{0}\right)>0$ given by Lemma 2.3 if $\epsilon_{0} \neq \infty$ and by Lemma 2.9 if $\epsilon_{0}=\infty$ and $\left(f_{0}, \delta_{0}\right) \neq\left(\mathfrak{p h}_{C_{0}}, 0\right)$; otherwise $c_{1}=\frac{1}{19}$;

- $c_{2}=c_{2}^{\prime}\left(\epsilon_{0}\right)>0$ given by Equation (4) if $\epsilon_{0} \neq \infty$ and by Equation (9) otherwise;

- $c_{3}=2 \sinh c_{1}+c_{2} e^{2 c_{1}} \sinh c_{1}$, which is positive, and depends on $\epsilon_{0}$;

- $c_{4}=c_{3}^{\prime}\left(\epsilon_{0}\right) \sinh \left(c_{1}+\delta_{0}\right)+c_{2} e^{-3 c_{3}^{\prime}\left(\epsilon_{0}\right) \sinh \left(c_{1}+\delta_{0}\right)-\log 2} \sinh c_{1}$, where $c_{3}^{\prime}(\cdot)$ is given by Equation (6) if $\epsilon_{0} \neq \infty$ and by Equation (11) otherwise. Note that $c_{4}$ is positive, and depends on $\epsilon_{0}, \delta_{0}$;

- $c_{5}=c_{5}\left(\epsilon_{0}, \delta_{0}\right)=2 \max \left\{c_{2}, c_{3}^{\prime}\left(\epsilon_{0}\right)\right\} \sinh \left(c_{1}+\delta_{0}\right)$, which is positive, and depends on $\epsilon_{0}, \delta_{0}$;

- $c_{6}=3 c_{4}+\log 2$, which is positive, and depends on $\epsilon_{0}, \delta_{0}$;

- $h_{0}=h_{0}\left(\epsilon_{0}, \delta_{0}, \kappa_{0}\right)=\max \left\{\delta_{0}+\kappa_{0}, c_{0}\left(\epsilon_{0}\right)+\kappa_{0}, h^{\prime}\left(\epsilon_{0}, \sinh \left(\delta_{0}+c_{1}\right)\right), \delta_{0}+\right.$ $\left.2 c_{1}+c_{6}, c_{6}+\kappa_{0}+\delta_{0}-2 c_{5}\right\}$, where $c_{0}(\cdot)$ is given by Equation (1) if $\epsilon_{0} \neq \infty$ and by Equation (8) otherwise, and $h^{\prime}(\cdot, \cdot)$ is given by Equation (5) if $\epsilon_{0} \neq \infty$ and by Equation (11) otherwise;

- For every $h_{0}^{\prime} \geq 0$, let $h_{1}^{\prime}=h_{1}^{\prime}\left(\epsilon_{0}, \delta_{0}, h_{0}^{\prime}\right)=h_{0}^{\prime}+2 c_{5}$.

Fix $h_{0}^{\prime} \geq h_{0}$ and $h \geq h_{1}^{\prime}$. 
Assumptions on the family $\left(C_{n}\right)_{n \in \mathbb{N}}$ Assume that there exists at least one geodesic ray or line $\gamma_{0}$ starting from $\xi_{0}$ with $f_{0}\left(\gamma_{0}\right)=h$ (this implies that $\gamma_{0}$ meets $C_{0}$, since $f_{0}$ is a $\kappa_{0}$-penetration map in $C_{0}$ and $h \geq h_{1}^{\prime} \geq h_{0}^{\prime} \geq h_{0}>\kappa_{0}$, hence $\ell_{C_{0}}\left(\gamma_{0}\right)>0$ ), and that the following conditions are satisfied.

(iii) (Almost disjointness property) For every $m, n$ in $\mathbb{N}$ with $m \neq n$, the diameter of $C_{n} \cap C_{m}$ is at most $\delta_{0}$.

(iv) (Local prescription property) For every $n$ in $\mathbb{N}-\{0\}$ such that $\xi_{0} \notin C_{n} \cup \partial_{\infty} C_{n}$, if there exists a geodesic ray or line $\alpha$ starting from $\xi_{0}$ which meets first $C_{0}$ and then $C_{n}$ with $f_{0}(\alpha)=h$ and $\ell_{C_{n}}(\alpha) \geq h_{0}^{\prime}$, then there exists a geodesic ray or line $\alpha^{\prime}$, starting from $\xi_{0}$ which meets first $C_{0}$ and then $C_{n}$ with $f_{0}\left(\alpha^{\prime}\right)=h$ and $\ell_{C_{n}}\left(\alpha^{\prime}\right)=h_{0}^{\prime}$.

Note that (iii) is satisfied with $\delta_{0}=0$ if the $C_{n}$ 's have disjoint interior. In Section 5, we will use Proposition 3.7 to check (iv) for various applications, with $h_{0}^{\prime}=\max \left\{h_{0}, h_{0}^{\min }\right\}$ and $h \geq \max \left\{h_{1}^{\prime}, h^{\min }\right\}$, for the various values of $h_{0}^{\min }, h^{\min }$ defined in Proposition 3.7. For every $n$ in $\mathbb{N}$ such that $\xi_{0} \notin C_{n} \cup \partial_{\infty} C_{n}$, define $f_{n}=\ell_{C_{n}}: T_{\xi_{0}}^{1} X \rightarrow[0,+\infty]$, and for every geodesic ray or line $\gamma$ starting from $\xi_{0}$ and meeting $C_{n}^{\xi 0}$, let $t_{n}^{-}(\gamma), t_{n}^{+}(\gamma) \in$ ]$-\infty,+\infty]$ be the entrance time and exit time of $\gamma$ in and out of the convex subset $C_{n}$ respectively. The following remark will be used later on.

Lemma 4.5 For every $n>0$, for every geodesic ray or line $\gamma$ starting from $\xi_{0}$ and entering $C_{0}$ at time $t=0$, such that $f_{0}(\gamma)=h$ and $\gamma(] \delta_{0},+\infty[)$ meets $C_{n}$, we have $\xi_{0} \notin C_{n} \cup \partial_{\infty} C_{n}$ and $t_{n}^{-}(\gamma)>0$.

Proof Otherwise, as $\gamma(] \delta_{0},+\infty[)$ meets $C_{n}$ and by convexity, there exists $\epsilon>0$ such that the geodesic segment $\gamma\left(\left[0, \delta_{0}+\epsilon\right]\right)$ is contained in $C_{n}$. By the penetration property (i) of $f_{0}$, the length of $\gamma \cap C_{0}$ is at least $h-\kappa_{0}$, which is bigger than $\delta_{0}$ as $h \geq h_{1}^{\prime}>h_{0}^{\prime} \geq h_{0} \geq \delta_{0}+\kappa_{0}$ by the definitions of $h_{1}^{\prime}$ and $h_{0}$. As $\gamma$ enters $C_{0}$ at time $t=0$, up to taking $\epsilon>0$ smaller, this implies that the geodesic segment $\gamma\left(\left[0, \delta_{0}+\epsilon\right]\right)$ is also contained in $C_{0}$. This contradicts the almost disjointness property (iii) as $n \neq 0$.

Statement of the inductive construction We will define by induction an initial segment $\mathcal{N}$ in $\mathbb{N}$, and finite or infinite sequences

- $\left(\gamma_{k}\right)_{k \in \mathcal{N}}$ of geodesic rays or lines starting from $\xi_{0}$,

- $\left(n_{k}\right)_{k \in \mathcal{N}}$ of integers such that $\xi_{0} \notin C_{n_{k}} \cup \partial_{\infty} C_{n_{k}}$,

- $\left(u_{k}\right)_{k \in \mathcal{N}}$ of maps $u_{k}:\left[0,+\infty\left[\rightarrow\left[h_{0}^{\prime}, h_{1}^{\prime}\right]\right.\right.$, 
such that the following assertions hold, for every $k$ in $\mathcal{N}$, where we use $t_{k}^{ \pm}=t_{n_{k}}^{ \pm}\left(\gamma_{k}\right)$ to simplify notation.

(1) The geodesic ray or line $\gamma_{k}$ enters $C_{0}$ at time $t=0$ and $f_{0}\left(\gamma_{k}\right)=h$.

(2) If $k \geq 1$, then $\gamma_{k}$ meets $C_{n_{k}}$ with $t_{k}^{-} \geq 0$ and $f_{n_{k}}\left(\gamma_{k}\right)=h_{0}^{\prime}$.

(3) If $k \geq 1$, then $d\left(\gamma_{k}(t), \gamma_{k-1}(t)\right) \leq c_{3} e^{t-t_{k}^{-}}$for every $t$ in $\left[0, t_{k}^{-}\right]$.

(4) If $k \geq 1$, then

$$
u_{k}(t)=\sup _{s \in\left[0,+\infty\left[:|s-t| \leq c_{4} e^{t-t_{k}^{-}}\right.\right.} u_{k-1}(s)+c_{5} e^{t-t_{k}^{-}}
$$

for $t \in\left[0, t_{k}^{-}\right]$and $u_{k}(t)=h_{0}^{\prime}$ if $t>t_{k}^{-}$.

(5) If $k \geq 1$, then $t_{k}^{-} \geq t_{k-1}^{-}+c_{6}$.

(6) If $k \geq 1$, for every $n$ in $\mathbb{N}-\{0\}$ such that $\gamma_{k}(] \delta_{0},+\infty[)$ meets $C_{n}$ with $t_{n}^{-}\left(\gamma_{k}\right) \leq$ $t_{k}^{-}$, we have $f_{n}\left(\gamma_{k}\right) \leq u_{k}\left(t_{n}^{+}\left(\gamma_{k}\right)-\delta_{0}\right)$.

Note that by Lemma 4.5 and by (1), if $\gamma_{k}(] \delta_{0},+\infty[)$ meets $C_{n}$ for some $n \geq 1$, then $\xi_{0} \notin C_{n} \cup \partial_{\infty} C_{n}$, so that, in particular, $t_{n}^{ \pm}\left(\gamma_{k}\right)$ are well defined, and (6) does make sense.

Proof of the inductive construction By the assumptions, let $\gamma_{0}$ be a geodesic ray or line starting from $\xi_{0}$ and entering $C_{0}$ at time $t_{0}^{-}=0$, such that $f_{0}\left(\gamma_{0}\right)=h$. Let $n_{0}=0$. Let $u_{0}:\left[0,+\infty\left[\rightarrow\left[h_{0}^{\prime}, h_{1}^{\prime}\right]\right.\right.$ be the constant map with value $h_{0}^{\prime}$. As the conditions (2)-(6) are empty if $k=0$, the construction is done at step 0 .

Let $k \geq 1$, and assume that $\gamma_{0}, n_{0}, u_{0}, \ldots, \gamma_{k-1}, n_{k-1}, u_{k-1}$ are constructed. Note that $u_{k-1} \geq h_{0}^{\prime}$ by induction. If for every $n$ in $\mathbb{N}-\{0\}$ such that $\gamma_{k-1}(] \delta_{0},+\infty[)$ meets $C_{n}$, we have $f_{n}\left(\gamma_{k-1}\right) \leq u_{k-1}\left(t_{n}^{+}\left(\gamma_{k-1}\right)-\delta_{0}\right)$, then we stop and we define $\mathcal{N}=\{0,1 \ldots, k-1\}$.

Otherwise, let $\tau$ be the greatest lower bound of the $t_{n}^{-}\left(\gamma_{k-1}\right)$ 's taken over all $n$ in $\mathbb{N}-\{0\}$ such that $\gamma_{k-1}(] \delta_{0},+\infty[)$ meets $C_{n}$ with $f_{n}\left(\gamma_{k-1}\right)>u_{k-1}\left(t_{n}^{+}\left(\gamma_{k-1}\right)-\delta_{0}\right)$.

Let us prove that this lower bound is in fact a minimum, attained for only one such $n$. Let $\epsilon>0$ such that $h_{0}^{\prime}>\delta_{0}+\epsilon$, which is possible by the definition of $h_{0}$, as $h_{0}^{\prime} \geq h_{0}$. If $t_{n}^{-}\left(\gamma_{k-1}\right)$ and $t_{m}^{-}\left(\gamma_{k-1}\right)$ belong to $[\tau, \tau+\epsilon]$ with $f_{n}\left(\gamma_{k-1}\right)>u_{k-1}\left(t_{n}^{+}\left(\gamma_{k-1}\right)-\delta_{0}\right)$ and $f_{m}\left(\gamma_{k-1}\right)>u_{k-1}\left(t_{m}^{+}\left(\gamma_{k-1}\right)-\delta_{0}\right)$, assume for instance that $t_{n}^{-}\left(\gamma_{k-1}\right) \leq t_{m}^{-}\left(\gamma_{k-1}\right)$. As $f_{n}=\ell_{C_{n}}, f_{m}=\ell_{C_{m}}, u_{k-1} \geq h_{0}^{\prime}$ and $t_{m}^{-}\left(\gamma_{k-1}\right)-t_{n}^{-}\left(\gamma_{k-1}\right) \leq \epsilon$, the subsets $C_{n}$ and $C_{m}$ meet along a segment of length at least $h_{0}^{\prime}-\epsilon>\delta_{0}$. By the almost disjointness property (iii), this implies that $n=m$. In particular, we have $\tau=t_{n}^{-}\left(\gamma_{k-1}\right)$ for a 
unique $n \in \mathbb{N}-\{0\}$, and we denote this $n$ by $n_{k} \in \mathbb{N}-\{0\}$, so that $\gamma_{k-1}(] \delta_{0},+\infty[)$ meets $C_{n_{k}}$ with

$$
f_{n_{k}}\left(\gamma_{k-1}\right)>u_{k-1}\left(t_{n_{k}}^{+}\left(\gamma_{k-1}\right)-\delta_{0}\right) \geq h_{0}^{\prime} .
$$

In particular, $\xi_{0} \notin C_{n_{k}} \cup \partial_{\infty} C_{n_{k}}$ by Lemma 4.5 and by Assertion (1) at rank $k-1$. Note that $n_{k} \neq n_{k-1}$, as $f_{n_{k-1}}\left(\gamma_{k-1}\right)=h_{0}^{\prime}$ by Assertion (2) at rank $k-1$, which would contradict Equation (16) if $n_{k}=n_{k-1}$.

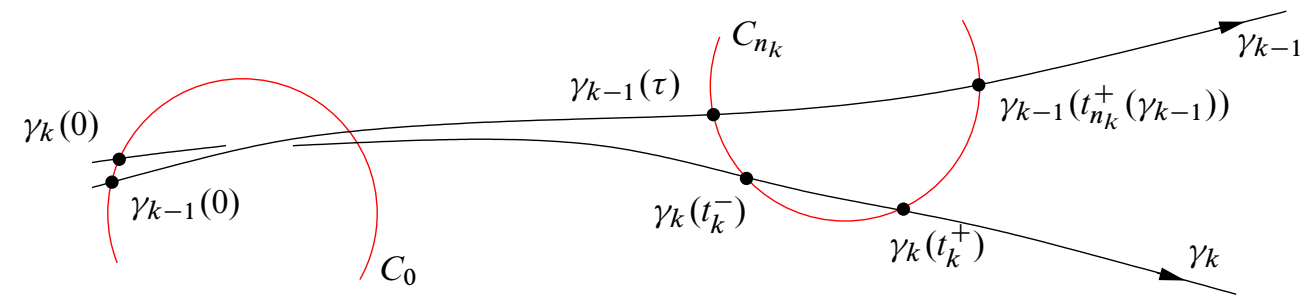

By Lemma 4.5, the geodesic ray or line $\gamma_{k-1}$ first enters $C_{0}$ and then $C_{n_{k}}$. Furthermore, $\gamma_{k-1}$ satisfies (1) and $f_{n_{k}}\left(\gamma_{k-1}\right) \geq h_{0}^{\prime}$. Hence, by the local prescription property (iv), there exists a geodesic ray or line $\gamma_{k}$ starting from $\xi_{0}$ that first enters $C_{0}$ and then $C_{n_{k}}$, with $f_{0}\left(\gamma_{k}\right)=h$ and $f_{n_{k}}\left(\gamma_{k}\right)=h_{0}^{\prime}$. Choose the parametrization in such a way that $\gamma_{k}$ enters $C_{0}$ at time 0 . In particular, (1) and (2) hold for $\gamma_{k}$, and $t_{k}^{-}=t_{n_{k}}^{-}\left(\gamma_{k}\right)>0$. Define $u_{k}:[0,+\infty[\rightarrow[0,+\infty[$ by using the induction formula given in Assertion (4). Before checking (3)-(6) for $\gamma_{k}, n_{k}, u_{k}$, let us make two preliminary remarks.

Lemma 4.6 We have $d\left(\gamma_{k-1}(\tau), \gamma_{k}\left(t_{k}^{-}\right)\right) \leq c_{1}$ and $d\left(\gamma_{k-1}(0), \gamma_{k}(0)\right) \leq c_{1}$.

Proof By Lemma 2.3 if $\epsilon_{0} \neq \infty$ and Lemma 2.9 otherwise, we have the inequalities $d\left(\gamma_{k-1}(\tau), \gamma_{k}\left(t_{k}^{-}\right)\right) \leq c_{1}^{\prime}\left(\epsilon_{0}\right)$ and $d\left(\gamma_{k-1}(0), \gamma_{k}(0)\right) \leq c_{1}^{\prime}\left(\epsilon_{0}\right)$. By the definition of $c_{1}$, we hence only have to prove Lemma 4.6 when $\epsilon_{0}=\infty, \delta_{0}=0$ and $f_{0}=\mathfrak{p h}_{C_{0}}$. In this case, as $c_{1}=1 / 19, c_{2}=5 / 2, c_{0}(\infty)=4.056, \kappa_{0}=2 \log (1+\sqrt{2})=c_{1}^{\prime}(\infty)$, $c_{3}^{\prime}(\infty)=5 / 2$, easy computations show that

$$
h_{0}=h^{\prime}\left(\infty, \sinh c_{1}\right)=3 \sinh c_{1}+c_{0}(\infty)+c_{1}^{\prime}(\infty) \approx 5.9767
$$

and, for future use,

$$
h_{1}^{\prime}\left(\infty, 0, h_{0}\left(\infty, 0, c_{1}^{\prime}(\infty)\right)\right) \approx 6.5032 .
$$

As $\mathfrak{p h} C_{C_{0}}\left(\gamma_{k}\right)$ and $\mathfrak{p h}_{C_{0}}\left(\gamma_{k-1}\right)$ are equal to $h \geq h_{1}^{\prime} \geq h_{0}^{\prime} \geq h_{0}$, and since $h_{0} / 2 \geq \log 2$, it follows from the definition of the map $\mathfrak{p h}_{C_{0}}$ and from Lemma 4.2 (1) and (2) that $d\left(\gamma_{k-1}(0), \gamma_{k}(0)\right)$ and similarly $d\left(\gamma_{k-1}(\tau), \gamma_{k}\left(t_{k}^{-}\right)\right)$are at most $v\left(h_{0} / 2\right)$, where $v(\cdot)$ is defined by Equation (14). An easy computation shows that $v\left(h_{0} / 2\right) \leq c_{1}=1 / 19$, which proves the result. 
Lemma 4.7 We have $\left|\tau-t_{k}^{-}\right| \leq 2 c_{1}$.

Proof Lemma 4.5, applied to $n=n_{k}$ and $\gamma=\gamma_{k-1}$, implies that $\tau>0$. We have seen that $t_{k}^{-}>0$. By the triangle inequality and the above lemma, we have $\left|\tau-t_{k}^{-}\right| \leq 2 c_{1}$.

Verification of (5) If $k=1$, then since $n_{1} \neq 0, t_{1}^{-}>0$, and $f_{n_{1}}\left(\gamma_{1}\right)=h_{0}^{\prime} \geq h_{0}>\delta_{0}$, we have $t_{1}^{-} \geq \ell_{C_{0}}\left(\gamma_{1}\right)-\delta_{0}$ by the almost disjointness property (iii). Therefore

$$
\begin{aligned}
t_{1}^{-}-t_{0}^{-} & =t_{1}^{-} \geq \ell_{C_{0}}\left(\gamma_{1}\right)-\delta_{0} \geq f_{0}\left(\gamma_{1}\right)-\delta_{0}-\kappa_{0}=h-\kappa_{0}-\delta_{0} \\
& \geq h_{1}^{\prime}-\kappa_{0}-\delta_{0}=h_{0}^{\prime}+2 c_{5}-\kappa_{0}-\delta_{0} \geq h_{0}+2 c_{5}-\kappa_{0}-\delta_{0} \geq c_{6},
\end{aligned}
$$

by the definition of $h_{0}$.

Assume now that $k \geq 2$. Note that $\tau=t_{n_{k}}^{-}\left(\gamma_{k-1}\right)>t_{k-1}^{-}$. Otherwise, as

$$
t_{n_{k}}^{+}\left(\gamma_{k-1}\right)=\tau+f_{n_{k}}\left(\gamma_{k-1}\right) \geq \tau+h_{0}^{\prime} \geq \tau+h_{0}>\delta_{0}
$$

by Equation (16) and by the definition of $h_{0}$, we have, by the assertion (6) at step $k-1$, the inequality $f_{n_{k}}\left(\gamma_{k-1}\right) \leq u_{k-1}\left(t_{n_{k}}^{+}\left(\gamma_{k-1}\right)-\delta_{0}\right)$, which contradicts the definition of $n_{k}$ by Equation (16).

Let us first prove that $\tau \geq t_{k-1}^{-}+h_{0}-\delta_{0}$. Assume first that $\tau \geq t_{k-1}^{+}$. Since $k \geq 2$, we have

$$
\tau-t_{k-1}^{-} \geq t_{k-1}^{+}-t_{k-1}^{-}=f_{n_{k-1}}\left(\gamma_{k-1}\right)=h_{0}^{\prime} \geq h_{0} .
$$

Hence the result holds. Otherwise, $t_{k-1}^{-}<\tau<t_{k-1}^{+}$. By convexity, $\gamma_{k-1}(\tau)$ belongs to $C_{n_{k-1}}$. Note that $\gamma_{k-1}\left(\left[\tau, \tau+h_{0}\right]\right)$ is contained in $C_{n_{k}}$, since $\tau$ is the entrance time of $\gamma_{k-1}$ in $C_{n_{k}}$, and $f_{n_{k}}\left(\gamma_{k-1}\right) \geq h_{0}^{\prime} \geq h_{0}$. If $\tau+\delta_{0}<t_{k-1}^{+}$, as $\ell_{C_{n_{k}}}\left(\gamma_{k-1}\right) \geq h_{0}>\delta_{0}$ by the definition of $h_{0}$, then $C_{n_{k}} \cap C_{n_{k-1}}$ contains a geodesic segment of length bigger than $\delta_{0}$. This contradicts the almost disjointness property (iii) since $n_{k} \neq n_{k-1}$. Hence

$$
\tau \geq t_{k-1}^{+}-\delta_{0} \geq t_{k-1}^{-}+f_{n_{k-1}}\left(\gamma_{k-1}\right)-\delta_{0} \geq t_{k-1}^{-}+h_{0}-\delta_{0}
$$

and the result holds.

Now, by Lemma 4.7,

$$
t_{k}^{-}-t_{k-1}^{-} \geq \tau-2 c_{1}-t_{k-1}^{-} \geq h_{0}-\delta_{0}-2 c_{1} \geq c_{6}
$$

by the definition of $h_{0}$. Therefore, Assertion (5) holds at rank $k$.

Verification of (4) We only have to check that $u_{k}$ has values in $\left[h_{0}^{\prime}, h_{1}^{\prime}\right]$. This will follow from the following easy but tedious general lemma. 
Lemma 4.8 Let $c, c^{\prime}, c^{\prime \prime}, h_{*} \geq 0$, let $\mathcal{M}$ be an initial segment in $\mathbb{N}$, let $\left(t_{n}\right)_{n \in \mathcal{M}}$ be a sequence of nonnegative real numbers, and let $\left(u_{n}:\left[0,+\infty\left[\rightarrow\left[0,+\infty[)_{n \in \mathcal{M}}\right.\right.\right.\right.$ be a sequence of maps. Assume that $u_{0}$ has constant value $h_{*}$, and that for every $n$ in $\mathcal{M}-\{0\}$, we have $t_{n}-t_{n-1} \geq c^{\prime \prime}, u_{n}(t)=h_{*}$ if $t>t_{n}$ and if $t \leq t_{n}$, then

$$
u_{n}(t)=c e^{t-t_{n}}+\sup _{s \in\left[0,+\infty\left[:|s-t| \leq c^{\prime} e^{t-t_{n}}\right.\right.} u_{n-1}(s) .
$$

If $c^{\prime \prime} \geq 3 c^{\prime}+\log 2$, then for every $t \in[0,+\infty[$, for every $n$ in $\mathcal{M}$, we have

$$
h_{*} \leq u_{n}(t) \leq h_{*}+2 c \text {. }
$$

To prove that $u_{k}$ has values in $\left[h_{0}^{\prime}, h_{1}^{\prime}\right]$, we apply Lemma 4.8 with $c=c_{5}, c^{\prime}=c_{4}$, $c^{\prime \prime}=c_{6}, h_{*}=h_{0}^{\prime}, \mathcal{M}=\{0,1, \ldots, k\}$ and $\left(t_{i}\right)_{i \in \mathcal{M}}=\left(t_{i}^{-}\right)_{1 \leq i \leq k}$. Its hypotheses are satisfied by the definition of the constant $c_{6}$, by Assertion (5) at rank less than or equal to $k$, that we just proved, and by the definition of $u_{k}$ and Assertion (4) for $u_{i}$ with $0 \leq 1 \leq k-1$. Hence the map $u_{k}$ does have values in $\left[h_{0}^{\prime}, h_{1}^{\prime}\right]$, by the definition of $h_{1}^{\prime}$.

Proof of Lemma 4.8 First note that by an easy induction, whatever the value of $c^{\prime \prime}$ is, for every $t \in\left[0,+\infty\left[\right.\right.$ and $n \in \mathcal{M}$, we have $u_{n}(t) \geq h_{*}$.

Let $c^{\prime \prime} \geq 3 c^{\prime}+\log 2, t \in\left[0,+\infty\left[\right.\right.$ and $n \in \mathcal{M}$. Let us prove that $u_{n}(t) \leq h_{*}+2 c$. We may assume that $t \leq t_{n}$ and that $n \geq 1$. Define $t_{-1}=-2 c^{\prime}-1$. Let $m$ be the unique element in $\mathcal{M}$ such that $t_{m-1}+2 c^{\prime}<t \leq t_{m}+2 c^{\prime}$. Set $N=n-m$, which is nonnegative (otherwise $n \leq m-1$ and $t_{n} \leq t_{m-1} \leq t_{m-1}+2 c^{\prime}<t_{n}$, a contradiction). Note that for every integer $k$ with $0 \leq k \leq N$, we have $t_{n-k}-t_{m} \geq(n-m-k) c^{\prime \prime}$ hence

$$
t-t_{n-k} \leq 2 c^{\prime}-(N-k) c^{\prime \prime} .
$$

Consider the finite sequence $\left(x_{k}\right)_{0 \leq k \leq N}$ defined by $x_{0}=0$ and

$$
x_{k+1}=x_{k}+e^{c^{\prime} x_{k}-(N-k) c^{\prime \prime}+2 c^{\prime}}
$$

for $0 \leq k \leq N-1$. Let us prove by induction on $k$ that $x_{k} \leq e^{-(N-k) c^{\prime \prime}}$, which in particular implies that

$$
x_{N} \leq 1 .
$$

Indeed, the result is true for $k=0$. Assume it to be true for some $k \leq N-1$. Then

$$
\begin{aligned}
x_{k+1} & \leq e^{-(N-k) c^{\prime \prime}}+e^{c^{\prime} e^{-(N-k) c^{\prime \prime}}-(N-k) c^{\prime \prime}+2 c^{\prime}} \\
& \leq e^{-(N-k-1) c^{\prime \prime}}\left(e^{-c^{\prime \prime}}+e^{-c^{\prime \prime}+3 c^{\prime}}\right) \leq e^{-(N-k-1) c^{\prime \prime}}
\end{aligned}
$$

as $c^{\prime \prime} \geq 3 c^{\prime}+\log 2 \geq \log \left(1+e^{3 c^{\prime}}\right)$. 
Let us now prove by induction on $k$ that, for $0 \leq k \leq N$, we have

$$
u_{n}(t) \leq \sup _{|s-t| \leq c^{\prime} x_{k}} u_{n-k}(s)+c x_{k} .
$$

This is true if $k=0$, assume it is true for some $k \leq N-1$. In particular, $n-k \geq 1$. For every $s \in\left[0,+\infty\left[\right.\right.$ such that $|s-t| \leq c^{\prime} x_{k}$, we have

$$
u_{n-k}(s) \leq \sup _{\left|s^{\prime}-s\right| \leq c^{\prime} e^{s-t_{n-k}}} u_{n-k-1}\left(s^{\prime}\right)+c e^{s-t_{n-k}}
$$

(this is true by definition if $s \leq t_{n-k}$, and also true otherwise as then $u_{n-k}(s)=h_{*}$ and $u_{n-k-1}\left(s^{\prime}\right) \geq h_{*}$ for every $\left.s^{\prime}\right)$. Hence by the triangle inequality and Equation (19),

$$
\begin{aligned}
u_{n}(t) & \leq \sup _{\left|s^{\prime}-t\right| \leq c^{\prime} x_{k}+c^{\prime} e^{t+c^{\prime} x_{k}-t_{n-k}} u_{n-k-1}\left(s^{\prime}\right)+c x_{k}+c e^{t+c^{\prime} x_{k}-t_{n-k}}} \sup u_{\left|s^{\prime}-t\right| \leq c^{\prime} x_{k}+c^{\prime} e^{c^{\prime} x_{k}+2 c^{\prime}-(N-k) c^{\prime \prime}}} u_{n-k-1}\left(s^{\prime}\right)+c x_{k}+c e^{c^{\prime} x_{k}+2 c^{\prime}-(N-k) c^{\prime \prime}} \\
& =\sup _{\left|s^{\prime}-t\right| \leq c^{\prime} x_{k+1}} u_{n-k-1}\left(s^{\prime}\right)+c x_{k+1},
\end{aligned}
$$

which proves the inductive formula (21).

Finally, let us prove that $u_{n}(t) \leq h_{*}+2 c$, which finishes the proof of the lemma. Take $k=N$ in the inductive formula (21), and note that $n-N=m$. For every $\epsilon>0$, let $s \in\left[0,+\infty\left[\right.\right.$ with $|s-t| \leq c^{\prime} x_{N}$ such that $\sup _{\left|s^{\prime}-t\right| \leq c^{\prime} x_{N}} u_{m}\left(s^{\prime}\right) \leq u_{m}(s)+\epsilon$. If $s>t_{m}$ or $m=0$, then $u_{m}(s)=h_{*}$, hence by the inequality (20),

$$
u_{n}(t) \leq \sup _{\left|s^{\prime}-t\right| \leq c^{\prime} x_{N}} u_{m}\left(s^{\prime}\right)+c x_{N} \leq h_{*}+\epsilon+c,
$$

and the result holds. Otherwise, $s \leq t_{m}$ and $m \geq 1$. For every $s^{\prime} \in[0,+\infty[$ such that $\left|s^{\prime}-s\right| \leq c^{\prime} e^{s-t_{m}}$, by Equation (20), we have $s^{\prime} \geq s-c^{\prime} \geq t-c^{\prime} x_{N}-c^{\prime} \geq t-2 c^{\prime}>t_{m-1}$. Again, the definition of $s$ and the inequality (20) gives

$$
\begin{aligned}
u_{n}(t) & \leq u_{m}(s)+\epsilon+c x_{N} \\
& =\sup _{\left|s^{\prime}-s\right| \leq c^{\prime} e^{s-t_{m}}} u_{m-1}\left(s^{\prime}\right)+c e^{s-t_{m}}+\epsilon+c x_{N} \leq \epsilon+h_{*}+2 c,
\end{aligned}
$$

and the result also holds.

Verification of (3) Let $t$ be in $\left[0, t_{k}^{-}\right]$. Recall that $d\left(\gamma_{k-1}(\tau), \gamma_{k}\left(t_{k}^{-}\right)\right) \leq c_{1}$ by Lemma 4.6. By Lemma 2.1 applied with $x=\gamma_{k-1}(\tau), y=\gamma_{k}\left(t_{k}^{-}\right), z=\xi_{0}$, we have $d\left(\gamma_{k-1}(0), \gamma_{k}\right) \leq e^{-\tau} \sinh c_{1}$. By the penetration property (i) of $f_{0}$ and the definition of $h_{0}$, we have

$$
\ell_{C_{0}}\left(\gamma_{k}\right) \geq f_{0}\left(\gamma_{k}\right)-\kappa_{0}=h-\kappa_{0} \geq h_{0}-\kappa_{0} \geq c_{0}\left(\epsilon_{0}\right) .
$$


Thus, by Lemma 2.5 if $\epsilon_{0} \neq \infty$ and by Lemma 2.11 if $\epsilon_{0}=\infty$, and by the definition of $c_{2}$, we have

$$
d\left(\gamma_{k-1}(0), \gamma_{k}(0)\right) \leq c_{2} e^{-\tau} \sinh c_{1} .
$$

We refer to Section 2.1 for the definition and properties of the map $\beta_{\xi_{0}}$. It follows from the inequality (22) that

$$
\begin{aligned}
\left|\beta_{\xi_{0}}\left(\gamma_{k-1}(t), \gamma_{k}(t)\right)\right| & =\left|\beta_{\xi_{0}}\left(\gamma_{k-1}(0), \gamma_{k}(0)\right)\right| \\
& \leq d\left(\gamma_{k-1}(0), \gamma_{k}(0)\right) \leq c_{2} e^{-\tau} \sinh c_{1} .
\end{aligned}
$$

For every $s$ in $\mathbb{R}$, let $\gamma_{k-1}\left(s^{\prime}\right)$ be the point on the geodesic line $\gamma_{k-1}$ such that the equality $\beta_{\xi_{0}}\left(\gamma_{k-1}\left(s^{\prime}\right), \gamma_{k}(s)\right)=0$ holds. For every point $p \in \gamma_{k-1}$, we have

$$
d\left(p, \gamma_{k-1}\left(t^{\prime}\right)\right)=\left|\beta_{\xi_{0}}\left(p, \gamma_{k-1}\left(t^{\prime}\right)\right)\right|=\left|\beta_{\xi_{0}}\left(p, \gamma_{k}(t)\right)\right| \leq d\left(p, \gamma_{k}(t)\right) .
$$

Using the triangle inequality with the point $p$ the closest to $\gamma_{k}(t)$ on $\gamma_{k-1}$, Lemma 2.1 and Lemma 4.6, we hence have the following inequalities

$$
\begin{aligned}
d\left(\gamma_{k}(t), \gamma_{k-1}\left(t^{\prime}\right)\right) & \leq 2 d\left(\gamma_{k}(t), \gamma_{k-1}\right) \leq 2 e^{t-t_{k}^{-}} \sinh d\left(\gamma_{k}\left(t_{k}^{-}\right), \gamma_{k-1}(\tau)\right) \\
& \leq 2 e^{t-t_{k}^{-}} \sinh c_{1} .
\end{aligned}
$$

Note that, using Equation (24) with $p=\gamma_{k-1}(t)$ and the inequalities (23),

$$
d\left(\gamma_{k-1}(t), \gamma_{k-1}\left(t^{\prime}\right)\right)=\left|\beta_{\xi_{0}}\left(\gamma_{k-1}(t), \gamma_{k}(t)\right)\right| \leq c_{2} e^{-\tau} \sinh c_{1} .
$$

Hence, by the inequality (25), we have

$$
\begin{aligned}
d\left(\gamma_{k}(t), \gamma_{k-1}(t)\right) & \leq d\left(\gamma_{k}(t), \gamma_{k-1}\left(t^{\prime}\right)\right)+d\left(\gamma_{k-1}\left(t^{\prime}\right), \gamma_{k-1}(t)\right) \\
& \leq 2 e^{t-t_{k}^{-}} \sinh c_{1}+c_{2} e^{-\tau} \sinh c_{1} .
\end{aligned}
$$

As $\tau \geq t_{k}^{-}-2 c_{1}$ by Lemma 4.7, and by the definition of $c_{3}$, we get

$$
d\left(\gamma_{k}(t), \gamma_{k-1}(t)\right) \leq c_{3} e^{t-t_{k}^{-}},
$$

which proves the assertion (3) at rank $k$.

Verification of (6) By contradiction, assume that there exists $n \in \mathbb{N}-\{0\}$ such that $\gamma_{k}(] \delta_{0},+\infty\left[\right.$ ) meets $C_{n}$ (so that in particular $\xi_{0} \notin C_{n} \cup \partial_{\infty} C_{n}$ by Lemma 4.5), with $t_{n}^{-}\left(\gamma_{k}\right) \leq t_{k}^{-}$and

$$
f_{n}\left(\gamma_{k}\right)>u_{k}\left(t_{n}^{+}\left(\gamma_{k}\right)-\delta_{0}\right) .
$$

To simplify notation, let $s_{k}^{ \pm}=t_{n}^{ \pm}\left(\gamma_{k}\right), x=\gamma_{k}\left(s_{k}^{-}\right), y=\gamma_{k}\left(s_{k}^{+}\right)$, and, as we will prove later on that $\gamma_{k-1}$ also meets $C_{n}$, let $s_{k-1}^{ \pm}=t_{n}^{ \pm}\left(\gamma_{k-1}\right), x^{\prime}=\gamma_{k-1}\left(s_{k-1}^{-}\right)$, $y^{\prime}=\gamma_{k-1}\left(s_{k-1}^{+}\right)$. 


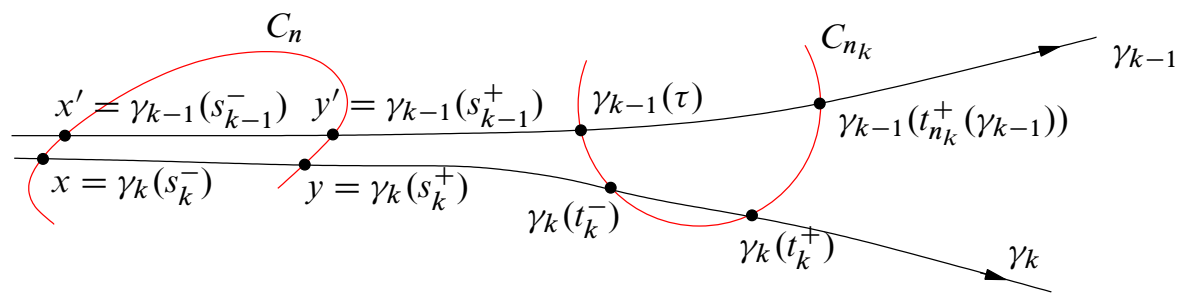

Note that $s_{k}^{+} \leq t_{k}^{-}+\delta_{0}$. Otherwise, as $s_{k}^{-} \leq t_{k}^{-}$and by convexity, there exists $\epsilon>0$ such that $\gamma_{k}\left(\left[t_{k}^{-}, t_{k}^{-}+\delta_{0}+\epsilon\right]\right)$ is contained in $C_{n}$. As $t_{k}^{+}-t_{k}^{-}=h_{0}^{\prime} \geq h_{0}>\delta_{0}$, up to making $\epsilon$ smaller, the geodesic segment $\gamma_{k}\left(\left[t_{k}^{-}, t_{k}^{-}+\delta_{0}+\epsilon\right]\right)$ is also contained in $C_{n_{k}}$. Hence $n$ is equal to $n_{k}$ by the almost disjointness property (iii). But $f_{n_{k}}\left(\gamma_{k}\right)=h_{0}^{\prime}$ and, by Equation (26), we have $f_{n}\left(\gamma_{k}\right)>u_{k}\left(s_{k}^{+}-\delta_{0}\right) \geq h_{0}^{\prime}$, so that $n$ cannot be equal to $n_{k}$.

By Lemma 2.1 applied to the geodesic triangle with vertices $\gamma_{k}\left(t_{k}^{-}+\delta_{0}\right), \gamma_{k-1}(\tau), \xi_{0}$, and as $d\left(\gamma_{k}\left(t_{k}^{-}\right), \gamma_{k-1}(\tau)\right) \leq c_{1}$ by Lemma 4.6, we have

$$
\begin{aligned}
d\left(y, \gamma_{k-1}\right) & \leq e^{-d\left(\gamma_{k}\left(t_{k}^{-}+\delta_{0}\right), y\right)} \sinh d\left(\gamma_{k}\left(t_{k}^{-}+\delta_{0}\right), \gamma_{k-1}(\tau)\right) \\
& \leq e^{s_{k}^{+}-t_{k}^{-}-\delta_{0}} \sinh \left(\delta_{0}+c_{1}\right)
\end{aligned}
$$

which is, in particular, at most $\sinh \left(\delta_{0}+c_{1}\right)$.

Note that

(28) $d(x, y)=f_{n}\left(\gamma_{k}\right)>h_{0}^{\prime} \geq h_{0} \geq h^{\prime}\left(\epsilon_{0}, \sinh \left(\delta_{0}+c_{1}\right)\right) \geq \sinh \left(\delta_{0}+c_{1}\right)+c_{1}^{\prime}\left(\epsilon_{0}\right)$,

by the definition of $h_{0}$ and of $h^{\prime}(\cdot, \cdot)$ in Equation (5) if $\epsilon_{0} \neq \infty$ and in Equation (11) otherwise. It follows from Lemma 2.6 if $\epsilon_{0} \neq \infty$ and by Lemma 2.12 otherwise, that the geodesic line $\gamma_{k-1}$ meets $C_{n}$ (thus $x^{\prime}$ and $y^{\prime}$ indeed exist) and that one of the following two assertions hold:

$$
d\left(y, y^{\prime}\right) \leq c_{3}^{\prime}\left(\epsilon_{0}\right) d\left(x^{\prime}, \gamma_{k}\right)
$$

or

$$
d\left(x^{\prime}, y^{\prime}\right) \geq d(x, y) .
$$

Note that $d\left(x, x^{\prime}\right) \leq c_{1}^{\prime}\left(\epsilon_{0}\right)$ by Lemma 2.3 if $\epsilon_{0} \neq \infty$ and Lemma 2.9 otherwise. Let $q^{\prime}$ be the closest point to $y$ on $\gamma_{k-1}$, so that

$$
d\left(y, q^{\prime}\right)=d\left(y, \gamma_{k-1}\right) \leq \sinh \left(\delta_{0}+c_{1}\right) .
$$

By the cocycle property of $\beta_{\xi_{0}}$, we have

$$
\beta_{\xi_{0}}\left(q^{\prime}, x^{\prime}\right)=\beta_{\xi_{0}}\left(q^{\prime}, y\right)+\beta_{\xi_{0}}(y, x)+\beta_{\xi_{0}}\left(x, x^{\prime}\right) \geq d(y, x)-d\left(y, q^{\prime}\right)-d\left(x, x^{\prime}\right),
$$


which is nonnegative by the two previous assertions and Equation (28). Hence $\xi_{0}, x^{\prime}, q^{\prime}$ are in this order on $\gamma_{k}$. Therefore, by convexity,

$$
d\left(x^{\prime}, \gamma_{k}\right) \leq d\left(q^{\prime}, \gamma_{k}\right) \leq d\left(q^{\prime}, y\right)=d\left(y, \gamma_{k-1}\right) .
$$

By Equation (28) and the definition of $h_{0}$, we have $d(x, y) \geq h_{0} \geq c_{0}\left(\epsilon_{0}\right)$. Hence, by Lemma 2.5 if $\epsilon_{0} \neq \infty$ and Lemma 2.11 otherwise, and by the inequalities (31) and (27), we have

$$
d\left(x, x^{\prime}\right) \leq c_{2} d\left(x^{\prime}, \gamma_{k}\right) \leq c_{2} e^{s_{k}^{+}-t_{k}^{-}-\delta_{0}} \sinh \left(\delta_{0}+c_{1}\right) .
$$

Before obtaining a contradiction from both assertions (29) and (30), we prove a technical result.

Lemma 4.9 We have $\delta_{0}<s_{k-1}^{-}<\tau$, so that the geodesic ray $\gamma_{k-1}(] \delta_{0},+\infty[)$ meets $C_{n}$ with $t_{n}^{-}\left(\gamma_{k-1}\right)<\tau$.

Proof Assume first by contradiction that $s_{k-1}^{-} \leq \delta_{0}$. If $\left.\left.s_{k-1}^{-} \in\right] 0, \delta_{0}\right]$, we have by the triangle inequality, Lemma 4.6 and the inequality (32),

$$
\begin{aligned}
s_{k}^{-} & =d\left(\gamma_{k}(0), \gamma_{k}\left(s_{k}^{-}\right)\right) \\
& \leq d\left(\gamma_{k}(0), \gamma_{k-1}(0)\right)+d\left(\gamma_{k-1}(0), \gamma_{k-1}\left(s_{k-1}^{-}\right)\right)+d\left(\gamma_{k-1}\left(s_{k-1}^{-}\right), \gamma_{k}\left(s_{k}^{-}\right)\right) \\
& \leq c_{1}+\delta_{0}+c_{2} \sinh \left(\delta_{0}+c_{1}\right) .
\end{aligned}
$$

Let $z_{0}$ and $z_{s_{k-1}^{-}}$be the closest points on $\gamma_{k}$ to $\gamma_{k-1}(0)$ and $x^{\prime}=\gamma_{k-1}\left(s_{k-1}^{-}\right)$, respectively. If $s_{k-1}^{-} \leq 0$, then as the closest point map does not increase distances, we have

$$
\begin{aligned}
s_{k}^{-} & =d\left(\gamma_{k}(0), \gamma_{k}\left(s_{k}^{-}\right)\right) \leq d\left(\gamma_{k}(0), z_{0}\right)+d\left(z_{0}, \gamma_{k}\left(s_{k}^{-}\right)\right) \\
& \leq d\left(\gamma_{k}(0), z_{0}\right)+d\left(z_{s_{k-1}^{-}}, \gamma_{k}\left(s_{k}^{-}\right)\right) \\
& \leq d\left(\gamma_{k}(0), \gamma_{k-1}(0)\right)+d\left(\gamma_{k-1}\left(s_{k-1}^{-}\right), \gamma_{k}\left(s_{k}^{-}\right)\right) \\
& \leq c_{1}+c_{2} \sinh \left(\delta_{0}+c_{1}\right) .
\end{aligned}
$$

Hence, by the definition of $c_{5}$ and as $c_{3}^{\prime}\left(\epsilon_{0}\right) \geq 1$ (see Equation (6) if $\epsilon_{0} \neq \infty$ or (11) otherwise), we have

$$
c_{5} \geq c_{2} \sinh \left(\delta_{0}+c_{1}\right)+c_{3}^{\prime}\left(\epsilon_{0}\right) \sinh \left(\delta_{0}+c_{1}\right) \geq c_{2} \sinh \left(\delta_{0}+c_{1}\right)+\delta_{0}+c_{1} \geq s_{k}^{-} .
$$

Now $\ell_{C_{n}}\left(\gamma_{k}\right) \geq h_{0}>\delta_{0}$ by the definition of $h_{0}$, and

$$
\ell_{C_{0}}\left(\gamma_{k}\right) \geq f_{0}\left(\gamma_{k}\right)-\kappa_{0}=h-\kappa_{0} \geq h_{1}^{\prime}-\kappa_{0} \geq h_{0}+2 c_{5}-\kappa_{0}>\delta_{0}+c_{5} \geq \delta_{0}+s_{k}^{-} .
$$


As the entrance time $s_{k}^{-}$of $\gamma_{k}$ in $C_{n}$ is positive by Lemma 4.5, this implies that $\operatorname{diam}\left(C_{0} \cap C_{n}\right)>\delta_{0}$. As $n \neq 0$, this contradicts the almost disjointness property (iii). Hence $\delta_{0}<s_{k-1}^{-}$.

Assume now by contradiction that $s_{k-1}^{-} \geq \tau$. Then as in the case $s_{k-1}^{-} \leq 0$, we get

$$
t_{k}^{-}-s_{k}^{-} \leq d\left(\gamma_{k-1}(\tau), \gamma_{k}\left(t_{k}^{-}\right)\right)+d\left(\gamma_{k-1}\left(s_{k-1}^{-}\right), \gamma_{k}\left(s_{k}^{-}\right)\right) \leq c_{1}+c_{1}^{\prime}\left(\epsilon_{0}\right),
$$

by Lemma 4.6, and by Lemma 2.3 if $\epsilon_{0} \neq \infty$ and Lemma 2.9 otherwise. We have seen in the inequalities (28) that

$$
h_{0} \geq \sinh \left(\delta_{0}+c_{1}\right)+c_{1}^{\prime}\left(\epsilon_{0}\right)>\delta_{0}+c_{1}+c_{1}^{\prime}\left(\epsilon_{0}\right) .
$$

Hence

$$
t_{k}^{-} \geq s_{k}^{+}-\delta_{0} \geq s_{k}^{-}+h_{0}-\delta_{0}>s_{k}^{-}+c_{1}+c_{1}^{\prime}\left(\epsilon_{0}\right),
$$

a contradiction to Equation (33). Hence $s_{k-1}^{-}<\tau$.

Assume first that the inequality (29) holds. As $s_{k}^{-} \geq 0$ by Lemma 4.5 and by the definition of $h_{0}$, we have

$$
s_{k}^{+}>h_{0}^{\prime}+s_{k}^{-} \geq h_{0} \geq \delta_{0}+2 c_{1}+c_{6} .
$$

Hence, as $t_{k}^{-} \leq \tau+2 c_{1}$ by Lemma 4.7, we have $e^{-c_{6}} e^{s_{k}^{+}-\delta_{0}-t_{k}^{-}} \geq e^{-\tau}$. By the definition of $c_{6}$ and of $c_{4}$, we have

$$
c_{6}=3 c_{4}+\log 2 \geq 3 c_{3}^{\prime}\left(\epsilon_{0}\right) \sinh \left(c_{1}+\delta_{0}\right)+\log 2 .
$$

By the triangle inequality since $s_{k-1}^{+} \geq 0$ by Lemma 4.9, by Equations (29), (31), (27) and (22), and by the definition of $c_{4}$, we have

$$
\begin{aligned}
\left|s_{k}^{+}-s_{k-1}^{+}\right| & \leq d\left(y, y^{\prime}\right)+d\left(\gamma_{k}(0), \gamma_{k-1}(0)\right) \\
& \leq c_{3}^{\prime}\left(\epsilon_{0}\right) e^{s_{k}^{+}-t_{k}^{-}-\delta_{0}} \sinh \left(\delta_{0}+c_{1}\right)+c_{2} e^{-\tau} \sinh c_{1} \\
& \leq c_{4} e^{s_{k}^{+}-\delta_{0}-t_{k}^{-}} .
\end{aligned}
$$

By the Lipschitz property (ii) of $f_{n}=\ell_{C_{n}}$ (as $n \neq 0$ ), by the inequalities (32), (29), (31) and (27), and by the definition of $c_{5}$, we have

$$
\left|f_{n}\left(\gamma_{k-1}\right)-f_{n}\left(\gamma_{k}\right)\right| \leq 2 \max \left\{d\left(x, x^{\prime}\right), d\left(y, y^{\prime}\right)\right\}
$$

$$
\begin{aligned}
& \leq 2 \max \left\{c_{2} e^{s_{k}^{+}-\delta_{0}-t_{k}^{-}} \sinh \left(\delta_{0}+c_{1}\right), c_{3}^{\prime}\left(\epsilon_{0}\right) e^{s_{k}^{+}-\delta_{0}-t_{k}^{-}} \sinh \left(\delta_{0}+c_{1}\right)\right\} \\
& \leq c_{5} e^{s_{k}^{+}-\delta_{0}-t_{k}^{-}} .
\end{aligned}
$$

By the inequalities (26) and (35), by the definition of $\tau$ and by Lemma 4.9, we have $u_{k}\left(s_{k}^{+}-\delta_{0}\right)<f_{n}\left(\gamma_{k}\right) \leq f_{n}\left(\gamma_{k-1}\right)+c_{5} e^{s_{k}^{+}-\delta_{0}-t_{k}^{-}} \leq u_{k-1}\left(s_{k-1}^{+}-\delta_{0}\right)+c_{5} e^{s_{k}^{+}-\delta_{0}-t_{k}^{-}}$. 
Assume now that the inequality (30) holds instead of the inequality (29). Then $f_{n}\left(\gamma_{k}\right) \leq$ $f_{n}\left(\gamma_{k-1}\right)$, so we again have that

$$
u_{k}\left(s_{k}^{+}-\delta_{0}\right)<u_{k-1}\left(s_{k-1}^{+}-\delta_{0}\right)+c_{5} e^{s_{k}^{+}-\delta_{0}-t_{k}^{-}} .
$$

As $\left|\left(s_{k-1}^{+}-\delta_{0}\right)-\left(s_{k}^{+}-\delta_{0}\right)\right| \leq c_{4} e^{s_{k}^{+}-\delta_{0}-t_{k}^{-}}$by the inequality (34), this contradicts the assertion (4) on the map $u_{k}$. Hence the assertion (6) at rank $k$ is verified.

The main corollary of the construction The above inductive construction will only be used in this paper through the following summarising statement.

Proposition 4.10 Let $X$ be a proper geodesic CAT $(-1)$ metric space. Let $\epsilon_{0}$ be in $\mathbb{R}_{+}^{*} \cup\{\infty\}, \delta_{0}, \kappa_{0} \geq 0$ and $\xi_{0} \in X \cup \partial_{\infty} X$. Let $h_{0}^{\prime} \geq h_{0}\left(\epsilon_{0}, \delta_{0}, \kappa_{0}\right)$ and $h \geq h_{1}^{\prime}=$ $h_{1}^{\prime}\left(\epsilon_{0}, \delta_{0}, h_{0}^{\prime}\right)$. Let $\left(C_{n}\right)_{n \in \mathbb{N}}$ be a collection of $\epsilon_{0}$-convex subsets of $X$ which satisfies the assertions (iii) and (iv), and with $\xi_{0} \notin C_{0} \cup \partial_{\infty} C_{0}$. Let $f_{0}: T_{\xi_{0}}^{1} X \rightarrow[0,+\infty]$ be a continuous $\kappa_{0}$-penetration map in $C_{0}$. Assume that there exists a geodesic ray or line $\gamma_{0}$ starting from $\xi_{0}$ with $f_{0}\left(\gamma_{0}\right)=h$. Then there exists a geodesic ray or line $\gamma_{\infty}$ starting from $\xi_{0}$, entering $C_{0}$ at time $t=0$ with $f_{0}\left(\gamma_{\infty}\right)=h$, such that $\ell_{C_{n}}\left(\gamma_{\infty}\right) \leq h_{1}^{\prime}$ for every $n$ in $\mathbb{N}-\{0\}$ such that $\gamma_{\infty}(] \delta_{0},+\infty[)$ meets $C_{n}$.

Proof Apply the main construction of the previous subsections with initial input a geodesic ray or line $\gamma_{0}$ entering $C_{0}$ at time $t=0$ with $f_{0}\left(\gamma_{0}\right)=h$, to get finite or infinite sequences $\left(\gamma_{k}\right)_{k \in \mathcal{N}},\left(n_{k}\right)_{k \in \mathcal{N}},\left(u_{k}\right)_{k \in \mathcal{N}}$ satisfying the assertions (1)-(6).

If $\mathcal{N}$ is finite, with maximum $N$, define $\gamma_{k}=\gamma_{N}$ for $k>N$. Then the sequence $\left(\gamma_{k}\right)_{k \in \mathbb{N}}$ converges to a geodesic ray or line $\gamma_{\infty}=\gamma_{N}$ in $T_{\xi_{0}}^{1} X$. If $\mathcal{N}$ is infinite, as $X$ is complete, it follows from the assertions (3) and (5), by an easy geometric series argument, that the sequence $\left(\gamma_{k}\right)_{k \in \mathcal{N}}$ converges in $T_{\xi_{0}}^{1} X$ to a geodesic ray or line $\gamma_{\infty}$ starting from $\xi_{0}$. Since $C_{0}$ is closed, the point $\gamma_{\infty}(0)$ belongs to $C_{0}$. If $\gamma_{\infty}$ does not enter in $C_{0}$ at time $t=0$, then there exists $\eta>0$ such that $\gamma_{\infty}(-2 \eta) \in C_{0}$. By the strict convexity of $C_{0}$, the geodesic segment $] \gamma_{\infty}(-2 \eta), \gamma_{\infty}(0)[$ is contained in the interior of $C_{0}$. Hence if $n$ is big enough, we have $\gamma_{n}(-\eta) \in C_{0}$, which is impossible. Hence $\gamma_{\infty}$ does enter in $C_{0}$ at time $t=0$. By the continuity of $f_{0}$ and the assertion (1), we have $f_{0}\left(\gamma_{\infty}\right)=h$.

Suppose by contradiction that there exists $n$ in $\mathbb{N}-\{0\}$ such that $\gamma_{\infty}(] \delta_{0},+\infty[)$ meets $C_{n}$ and $\ell_{C_{n}}\left(\gamma_{\infty}\right)>h_{1}^{\prime}>0$. In particular, $\gamma_{\infty}(] \delta_{0},+\infty[)$ meets the interior of $C_{n}$ and $\xi_{0} \notin C_{n} \cup \partial_{\infty} C_{n}$ by Lemma 4.5. Furthermore, it follows from the definition of the stopping time, and the fact that $u_{k} \leq h_{1}^{\prime}$ for every $k$, that $\mathcal{N}$ is infinite. Hence, as the $\gamma_{k}$ 's converge to $\gamma_{\infty}$, and by the continuity of $\ell_{C_{n}}$, if $k$ is big enough, then $\gamma_{k}(] \delta_{0},+\infty[)$ meets $C_{n}$ and $\ell_{C_{n}}\left(\gamma_{k}\right)>h_{1}^{\prime}$. 
In particular, $t_{n}^{+}\left(\gamma_{k}\right)>\delta_{0}$. Since the point $\gamma_{k}\left(t_{n}^{-}\left(\gamma_{k}\right)\right)$ is at distance at most $c_{1}^{\prime}\left(\epsilon_{0}\right)$ from the point $\gamma_{\infty}\left(t_{n}^{-}\left(\gamma_{\infty}\right)\right)$ by Lemma 2.3 if $\epsilon_{0} \neq \infty$ and by Lemma 2.9 otherwise, the sequence of times $\left(t_{n}^{-}\left(\gamma_{k}\right)\right)_{k \in \mathbb{N}}$ is bounded. Hence if $k$ is big enough, then $t_{n}^{-}\left(\gamma_{k}\right)$ is less than $t_{k}^{-}$, since $t_{k}^{-}$converges to $+\infty$ when $k \rightarrow+\infty$ by the assertion (5). This contradicts the assertion (6), as $u_{k} \leq h_{1}^{\prime}$.

Remark If $X, \epsilon_{0}, \delta_{0}, \kappa_{0}, \xi_{0}, h_{0}^{\prime}, h,\left(C_{n}\right)_{n \in \mathbb{N}}, f_{0}$ satisfy the hypotheses in the statement of Proposition 4.10, and if for every $n$ such that $\xi_{0} \notin C_{n} \cup \partial_{\infty} C_{n}$, we have a $\kappa$-penetration map $g_{n}: T_{\xi_{0}}^{1} X \rightarrow[0,+\infty]$ for some constant $\kappa \geq 0$, then Proposition 4.10 implies that there exists a geodesic ray or line $\gamma_{\infty}$ starting from $\xi_{0}$, entering $C_{0}$ at time $t=0$ with $f_{0}\left(\gamma_{\infty}\right)=h$, such that $g_{n}\left(\gamma_{\infty}\right) \leq h_{1}^{\prime}+\kappa$ for every $n$ in $\mathbb{N}-\{0\}$ such that $\gamma_{\infty}(] \delta_{0},+\infty[)$ meets $C_{n}$. We will apply this observation to more general penetration maps than the $\ell_{C_{n}}$ 's, in Section 5 .

The next corollary yields geodesic lines with a prescribed penetration in $C_{0}$, and that essentially avoid the $C_{n}$ 's not only for positive times, but also for negative ones. The penetration in the sets $C_{n}$ for $n \neq 0$ cannot be made quite as small as in Proposition 4.10 .

Corollary 4.11 Let $X$ be a proper geodesic CAT $(-1)$ metric space. Let $\epsilon_{0}$ be in $\mathbb{R}_{+}^{*} \cup\{\infty\}$ and $\delta_{0}, \kappa_{0} \geq 0$. Let $C_{0}$ be a proper $\epsilon_{0}$-convex subset of $X$, and let

$$
f_{0}: \bigcup_{\xi \in \partial_{\infty} X-\partial_{\infty} C_{0}} T_{\xi}^{1} X \rightarrow[0,+\infty]
$$

be a continuous map such that $\left.f_{0}\right|_{\xi_{0}} ^{1} X$ is a $\kappa_{0}$-penetration map in $C_{0}$ for every $\xi_{0} \in \partial_{\infty} X-\partial_{\infty} C_{0}$. Let $h_{0}^{\prime} \geq h_{0}=h_{0}\left(\epsilon_{0}, \delta_{0}, \kappa_{0}\right), h \geq h_{1}^{\prime}=h_{1}^{\prime}\left(\epsilon_{0}, \delta_{0}, h_{0}^{\prime}\right)$, and

$$
h_{1}^{\prime \prime}=h_{1}^{\prime}\left(\epsilon_{0}, \delta_{0}, h_{0}^{\prime}\right)+c_{3}^{\prime}\left(\epsilon_{0}\right)\left(\delta_{0}+c_{1}\right)+c_{1}^{\prime}\left(\epsilon_{0}\right) .
$$

Assume that there exists a geodesic line $\gamma_{0}$ in $X$ with $f_{0}\left(\gamma_{0}\right)=h$. For every $n$ in $\mathbb{N}-\{0\}$, let $C_{n}$ be an $\epsilon_{0}$-convex subset of $X$, such that $\left(C_{n}\right)_{n \in \mathbb{N}}$ satisfies the assertions (iii) and (iv) with respect to every $\xi_{0} \in \partial_{\infty} X-\partial_{\infty} C_{0}$. Then there exists a geodesic line $\gamma^{\prime}$ in $X$ entering $C_{0}$ at time $t=0$ with $f_{0}\left(\gamma^{\prime}\right)=h$, such that $\ell_{C_{n}}\left(\gamma^{\prime}\right) \leq h_{1}^{\prime \prime}$ for every $n$ in $\mathbb{N}-\{0\}$.

Proof Let $\gamma_{0}$ be a geodesic line in $X$ with $f_{0}\left(\gamma_{0}\right)=h$, and let $\xi$ be the starting point at infinity of $\gamma_{0}$, which does not belong to $\partial_{\infty} C_{0}$ as $h<+\infty$. Applying Proposition 4.10 with $\xi_{0}=\xi$, as $h \geq h_{1}^{\prime}$, there exists a geodesic line $\gamma$ starting from $\xi$ and entering $C_{0}$ at time 0 , such that $f_{0}(\gamma)=h$ and $\ell_{C_{n}}(\gamma) \leq h_{1}^{\prime}$ for every $n \in \mathbb{N}-\{0\}$ such that $\gamma(] \delta_{0},+\infty[)$ meets $C_{n}$. 
Let $\xi^{\prime}$ be the other endpoint at infinity of $\gamma$, which does not belong to $\partial_{\infty} C_{0}$ as $h<+\infty$. Applying Proposition 4.10 again with now $\xi_{0}=\xi^{\prime}$, we get that there exists a geodesic line $\gamma^{\prime}$ starting from $\xi^{\prime}$ and entering $C_{0}$ at time 0 , such that $f_{0}\left(\gamma^{\prime}\right)=h$ and $\ell_{C_{n}}\left(\gamma^{\prime}\right) \leq h_{1}^{\prime}$ for every $n \in \mathbb{N}-\{0\}$ such that $\gamma^{\prime}(] \delta_{0},+\infty[)$ meets $C_{n}$.

Assume by contradiction that there exists $n \in \mathbb{N}-\{0\}$ such that $\ell_{C_{n}}\left(\gamma^{\prime}\right)>h_{1}^{\prime \prime}>0$. Then $\gamma^{\prime}$ enters $C_{n}$ at a point $x_{n}^{\prime}$, exiting it at a point $y_{n}^{\prime}$ at time at most $\delta_{0}$, as $h_{1}^{\prime \prime}>h_{1}^{\prime}$ by the definition of $h_{1}^{\prime \prime}$. In particular, if $x^{\prime}=\gamma^{\prime}(0)$ is the entering point of $\gamma^{\prime}$ in $C_{0}$, then $d\left(y_{n}^{\prime}, x^{\prime}\right) \leq \delta_{0}$ if $x^{\prime}, y_{n}^{\prime}, x_{n}^{\prime}, \xi^{\prime}$ are not in this order on $\gamma^{\prime}$.

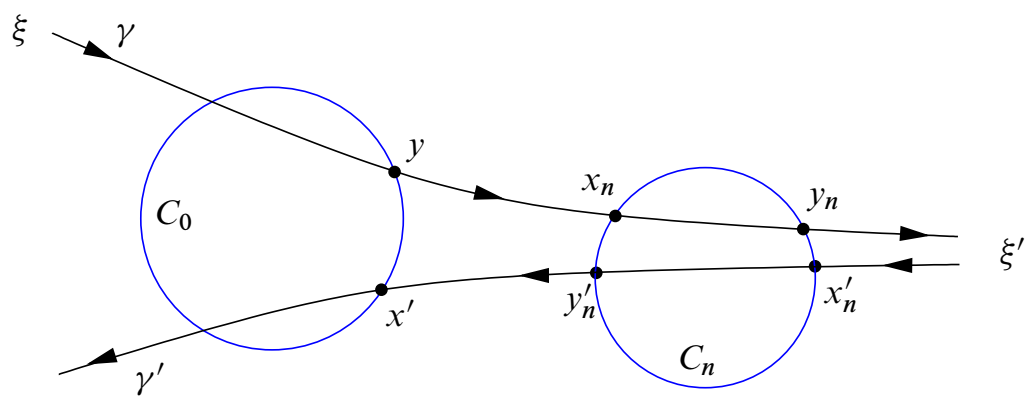

Let $y$ be the exiting point of $\gamma$ out of $C_{0}$. Note that

$$
h \geq h_{1}^{\prime} \geq h_{0}^{\prime} \geq h_{0} \geq h^{\prime}\left(\epsilon_{0}, \sinh \left(\delta_{0}+c_{1}\right)\right) \geq h^{\prime}\left(\epsilon_{0}, \delta_{0}+c_{1}\right)
$$

by the definitions of $h_{1}^{\prime}, h_{0}, h^{\prime}$. By Lemma 2.3 if $\epsilon_{0} \neq \infty$ and by Lemma 2.9 if $\epsilon_{0}=\infty$ and $\left(f_{0}, \delta_{0}\right) \neq\left(\mathfrak{p h}_{C_{0}}, 0\right)$, and as in the proof of Lemma 4.6 if $\left(\epsilon_{0}, f_{0}, \delta_{0}\right)=$ $\left(\infty, \mathfrak{p h}_{C_{0}}, 0\right)$ since $h \geq h_{0}$, we have $d\left(x^{\prime}, y\right) \leq c_{1}$. Hence by convexity,

$$
d\left(y_{n}^{\prime}, \gamma\right) \leq d\left(x^{\prime}, \gamma\right)+\delta_{0} \leq d\left(x^{\prime}, y\right)+\delta_{0} \leq \delta_{0}+c_{1} .
$$

Note that

$$
d\left(x_{n}^{\prime}, y_{n}^{\prime}\right)=\ell_{C_{n}}\left(\gamma^{\prime}\right)>h_{1}^{\prime \prime} \geq h_{1}^{\prime} \geq h^{\prime}\left(\epsilon_{0}, \delta_{0}+c_{1}\right)
$$

by the definition of $h_{1}^{\prime \prime}$ and by the inequalities (36). Hence, by Lemma 2.6 if $\epsilon_{0} \neq \infty$ and by Lemma 2.12 otherwise, the geodesic line $\gamma$ enters $C_{n}$ at a point $x_{n}$ and exits it at a point $y_{n}$ such that

$$
d\left(y_{n}^{\prime}, x_{n}\right) \leq c_{3}^{\prime}\left(\epsilon_{0}\right) d\left(y_{n}^{\prime}, \gamma\right) \quad \text { or } \quad d\left(x_{n}, y_{n}\right) \geq d\left(x_{n}^{\prime}, y_{n}^{\prime}\right) .
$$

Let us prove by contradiction that $\gamma(] \delta_{0},+\infty[)$ meets $C_{n}$. Otherwise, since $\gamma^{-1}(y) \geq 0$, by convexity, and by Lemma 2.3 if $\epsilon_{0} \neq \infty$ or Lemma 2.9 if $\epsilon_{0}=\infty$, we have

$$
d\left(x^{\prime}, x_{n}^{\prime}\right) \leq d\left(x^{\prime}, y\right)+\delta_{0}+d\left(y_{n}, x_{n}^{\prime}\right) \leq c_{1}+\delta_{0}+c_{1}^{\prime}\left(\epsilon_{0}\right) .
$$


By the definition of $h_{1}^{\prime \prime}$ and since $c_{3}^{\prime}\left(\epsilon_{0}\right) \geq 2$ by Equation (6) if $\epsilon_{0} \neq \infty$, and by Equation (11) otherwise, we have

$$
d\left(x^{\prime}, x_{n}^{\prime}\right) \geq d\left(x_{n}^{\prime}, y_{n}^{\prime}\right)-\delta_{0}>h_{1}^{\prime \prime}-\delta_{0} \geq h_{1}^{\prime}+2\left(\delta_{0}+c_{1}\right)+c_{1}^{\prime}\left(\epsilon_{0}\right)-\delta_{0} \geq c_{1}+\delta_{0}+c_{1}^{\prime}\left(\epsilon_{0}\right) \text {. }
$$

This contradicts the inequalities (39).

Assume first that the second of the inequalities (38) holds. As $d\left(x_{n}^{\prime}, y_{n}^{\prime}\right)>h_{1}^{\prime}$, this contradicts the construction of $\gamma$. Hence the first of the inequalities (38) is satisfied, and by Equation (37), we have

$$
d\left(y_{n}^{\prime}, x_{n}\right) \leq c_{3}^{\prime}\left(\epsilon_{0}\right) d\left(y_{n}^{\prime}, \gamma\right) \leq c_{3}^{\prime}\left(\epsilon_{0}\right)\left(\delta_{0}+c_{1}\right) .
$$

But then, by the triangle inequality and by the definition of $h_{1}^{\prime \prime}$, $d\left(x_{n}, y_{n}\right) \geq d\left(x_{n}^{\prime}, y_{n}^{\prime}\right)-d\left(x_{n}, y_{n}^{\prime}\right)-d\left(y_{n}, x_{n}^{\prime}\right)>h_{1}^{\prime \prime}-c_{3}^{\prime}\left(\epsilon_{0}\right)\left(\delta_{0}+c_{1}\right)-c_{1}^{\prime}\left(\epsilon_{0}\right)=h_{1}^{\prime}$, which contradicts the construction of $\gamma$.

\section{Prescribing the penetration of geodesic lines}

In this section, we apply Proposition 4.10 to prove a number of results on the geodesic flow of negatively curved Riemannian manifolds.

The following constants appear in the theorems, depending on $\in \in \mathbb{R}_{+}^{*} \cup\{\infty\}, \delta, \kappa \geq 0$.

- $c_{1}^{\prime \prime}(\epsilon, \delta, \kappa)=\max \left\{2 c_{1}^{\prime}(\epsilon)+2 \delta+\kappa, h_{1}^{\prime}\left(\epsilon, \delta, h_{0}\left(\epsilon, \delta, c_{1}^{\prime}(\infty)\right)\right)\right\}$.

- $c_{2}^{\prime \prime}(\epsilon)=c_{1}^{\prime \prime}(\epsilon, 0,0)+c_{1}^{\prime}(\infty)+2 c_{1}$, where $c_{1}=c_{1}^{\prime}(\epsilon)$ if $\epsilon \neq \infty$, and $c_{1}=1 / 19$ otherwise. Note that $c_{2}^{\prime \prime}(\infty)=h_{1}^{\prime}\left(\infty, 0, h_{0}\left(\infty, 0, c_{1}^{\prime}(\infty)\right)\right)+c_{1}^{\prime}(\infty)+2 c_{1} \approx$ 8.3712 by the definition of $c_{1}^{\prime \prime}(\epsilon, \delta, \kappa)$ and the approximation (17).

Recall that the constants $c_{1}^{\prime}(\epsilon)$ are given by Lemma 2.3 and Lemma 2.9, and that $h_{0}(\cdot, \cdot, \cdot)$ and $h_{1}^{\prime}(\cdot, \cdot, \cdot)$ are given in the list of constants in the beginning of Section 4.2.

\subsection{Climbing in balls and horoballs}

In this subsection, we construct geodesic rays or lines having prescribed penetration properties in a ball or a horoball, while essentially avoiding a family of almost disjoint convex subsets. Let us consider the penetration height and inner projection penetration maps first in horoballs and then in balls. Note that in these cases, if $f_{0}=\mathfrak{p h}_{C_{0}}$, then $\left\|f_{0}-\mathfrak{p h}_{C_{0}}\right\|_{\infty}=0$ and if $f_{0}=\mathfrak{i p p}_{C_{0}}$, then $\left\|f_{0}-\mathfrak{p h}_{C_{0}}\right\|_{\infty} \leq c_{1}^{\prime}(\infty)$ by Section 3.1. 
Theorem 5.1 Let $\epsilon \in \mathbb{R}_{+}^{*} \cup\{\infty\}, \delta, \kappa \geq 0$; let $X$ be a complete simply connected Riemannian manifold with sectional curvature at most -1 and dimension at least 3 ; let $\xi_{0} \in X \cup \partial_{\infty} X$; let $C_{0}$ be a horoball such that $\xi_{0} \notin C_{0} \cup \partial_{\infty} C_{0}$; let $f_{0}=\mathfrak{p h}_{C_{0}}$ or $f_{0}=\mathfrak{i p p}_{C_{0}}$; let $\left(C_{n}\right)_{n \in \mathbb{N}-\{0\}}$ be a family of $\epsilon$-convex subsets of $X$; for every $n \in \mathbb{N}-\{0\}$ such that $\xi_{0} \notin C_{n} \cup \partial_{\infty} C_{n}$, let $f_{n}: T_{\xi_{0}}^{1} X \rightarrow[0,+\infty]$ be a $\kappa$-penetration map in $C_{n}$. If $\operatorname{diam}\left(C_{n} \cap C_{m}\right) \leq \delta$ for all $n, m$ in $\mathbb{N}$ with $n \neq m$, then, for every $h \geq c_{1}^{\prime \prime}\left(\epsilon, \delta,\left\|f_{0}-\mathfrak{p h}_{C_{0}}\right\|_{\infty}\right)$, there exists a geodesic ray or line $\gamma$ starting from $\xi_{0}$ and entering $C_{0}$ at time 0 , such that $f_{0}(\gamma)=h$ and $f_{n}(\gamma) \leq c_{1}^{\prime \prime}\left(\epsilon, \delta,\left\|f_{0}-\mathfrak{p h} C_{0}\right\|_{\infty}\right)+\kappa$ for every $n \geq 1$ such that $\gamma(] \delta,+\infty[)$ meets $C_{n}$.

Proof Let $h \geq c_{1}^{\prime \prime}=c_{1}^{\prime \prime}\left(\epsilon, \delta,\left\|f_{0}-\mathfrak{p h} C_{C_{0}}\right\|_{\infty}\right)$. In order to apply Proposition 4.10, define $\epsilon_{0}=\epsilon, \delta_{0}=\delta, \kappa_{0}=c_{1}^{\prime}(\infty)=2 \log (1+\sqrt{2})$ and $h_{0}^{\prime}=h_{0}\left(\epsilon_{0}, \delta_{0}, \kappa_{0}\right)$. Recall that $\mathfrak{p h}_{C_{0}}$ and $\mathfrak{i p p}_{C_{0}}$ are $\kappa_{0}$-penetration maps for $C_{0}$ by Lemma 3.3. For every $n \in \mathbb{N}-\{0\}$ such that $\xi_{0} \notin C_{n} \cup \partial_{\infty} C_{n}$, let us apply Proposition 3.7 (1) to $C=C_{0}, C^{\prime}=C_{n}, f=f_{0}$, $f^{\prime}=\ell_{C_{n}}, h^{\prime}=h_{0}^{\prime}$, so that $h^{\min }=2 c_{1}^{\prime}(\epsilon)+2 \delta+\left\|f_{0}-\mathfrak{p h}_{C_{0}}\right\|_{\infty}$ and $h_{0}^{\min }=2 \delta$. Note that $h_{0}^{\min } \leq h_{0}^{\prime}$, as

$$
h_{0}^{\prime} \geq h^{\prime}\left(\epsilon, \sinh \left(\delta+c_{1}\right)\right) \geq 2 \sinh \left(\delta+c_{1}\right) \geq 2 \delta,
$$

by the definitions of $h_{0}$ and of $h^{\prime}(\cdot, \cdot)$. As $h \geq c_{1}^{\prime \prime} \geq h^{\text {min }}$ by the definition of $c_{1}^{\prime \prime}$, Proposition 3.7 (1) hence implies that $\left(C_{n}\right)_{n \in \mathbb{N}}$ satisfies the local prescription property (iv). Thus by Proposition 4.10, there exists a geodesic ray or line $\gamma$ starting at $\xi_{0}$ such that $f_{0}(\gamma)=h$ and $\ell_{C_{n}}(\gamma) \leq h_{1}^{\prime}\left(\epsilon_{0}, \delta_{0}, h_{0}\left(\epsilon_{0}, \delta_{0}, \kappa_{0}\right)\right)$, which implies that $f_{n}(\gamma) \leq c_{1}^{\prime \prime}+\kappa$, for every $n \geq 1$ such that $\gamma(] \delta,+\infty[)$ meets $C_{n}$.

The proof of the corresponding result when $C_{0}$ is a ball of radius $R \geq \epsilon$ is the same, using (2) of Proposition 3.7 instead of (1). This requires $h \leq h^{\max }=2 R-2 c_{1}^{\prime}(\epsilon)-$ $\left\|f_{0}-\mathfrak{p h} C_{C_{0}}\right\|_{\infty}$. To be nonempty, the following result requires

$$
R \geq c_{1}^{\prime \prime}\left(\epsilon, \delta,\left\|f_{0}-\mathfrak{p h}_{C_{0}}\right\|_{\infty}\right) / 2+c_{1}^{\prime}(\epsilon)+\left\|f_{0}-\mathfrak{p h}_{C_{0}}\right\|_{\infty} / 2 .
$$

Theorem 5.2 Let $\epsilon>0, \delta, \kappa \geq 0$; let $X$ be a complete simply connected Riemannian manifold with sectional curvature at most -1 and dimension at least 3 ; let $C_{0}$ be a ball of radius $R \geq \epsilon$; let $\xi_{0} \in\left(X \cup \partial_{\infty} X\right)-C_{0}$; let $f_{0}=\mathfrak{p h}_{C_{0}}$ or $f_{0}=\mathfrak{i p p}_{C_{0}}$; let $\left(C_{n}\right)_{n \in \mathbb{N}-\{0\}}$ be a family of $\epsilon$-convex subsets of $X$; for every $n \in \mathbb{N}-\{0\}$ such that $\xi_{0} \notin C_{n} \cup \partial_{\infty} C_{n}$, let $f_{n}: T_{\xi_{0}}^{1} X \rightarrow[0,+\infty]$ be a $\kappa$-penetration map in $C_{n}$. If $\operatorname{diam}\left(C_{n} \cap C_{m}\right) \leq \delta$ for all $n, m$ in $\mathbb{N}$ with $n \neq m$, then, for every

$$
h \in\left[c_{1}^{\prime \prime}\left(\epsilon, \delta,\left\|f_{0}-\mathfrak{p h} C_{0}\right\|_{\infty}\right), 2 R-2 c_{1}^{\prime}(\epsilon)-\left\|f_{0}-\mathfrak{p h} C_{0}\right\|_{\infty}\right],
$$

there exists a geodesic ray or line $\gamma$ starting from $\xi_{0}$ and entering $C_{0}$ at time 0 , such that $f_{0}(\gamma)=h$ and $f_{n}(\gamma) \leq c_{1}^{\prime \prime}\left(\epsilon, \delta,\left\|f_{0}-\mathfrak{p h}_{C_{0}}\right\|_{\infty}\right)+\kappa$ for every $n \geq 1$ such that $\gamma(] \delta,+\infty[)$ meets $C_{n}$. 
Varying the family $\left(C_{n}\right)_{n \in \mathbb{N}-\{0\}}$ of $\epsilon$-convex subsets appearing in Theorem 5.1 and Theorem 5.2, among balls of radius at least $\epsilon$, horoballs, $\epsilon$-neighbourhoods of totally geodesic subspaces, etc, we get several corollaries. We will only state two of them, Corollaries 5.3 and 5.5, which have applications to equivariant families. The proofs of these results are simplified versions of the proof of Corollary 4.11, giving better (though very probably not optimal) constants.

Corollary 5.3 Let $X$ be a complete simply connected Riemannian manifold with sectional curvature at most -1 and dimension at least 3 , and let $\left(H_{n}\right)_{n \in \mathbb{N}}$ be a family of horoballs in $X$ with pairwise disjoint interiors. Then, for every $h \geq c_{1}^{\prime \prime}(\infty, 0,0) \approx$ 6.5032, there exists a geodesic line $\gamma^{\prime}$ such that $\mathfrak{p h}_{H_{0}}\left(\gamma^{\prime}\right)=h$ and $\mathfrak{p h}_{H_{n}}\left(\gamma^{\prime}\right) \leq$ $c_{2}^{\prime \prime}(\infty) \approx 8.3712$ for every $n \geq 1$.

Proof Let $c_{1}^{\prime \prime}=c_{1}^{\prime \prime}(\infty, 0,0)$ and $c_{2}^{\prime \prime}=c_{2}^{\prime \prime}(\infty)$. Let $C_{0}=H_{0}$ and let $\xi$ be a point in $\partial_{\infty} X-\partial_{\infty} C_{0}$. We apply Theorem 5.1 with $\epsilon=\infty, \delta=0, \kappa=0, \xi_{0}=\xi, C_{n}=H_{n}$ for every $n$ in $\mathbb{N}, f_{0}=\mathfrak{p h}_{C_{0}}$, and $f_{n}=\ell_{C_{n}}$ for every $n \neq 0$ such that $\xi_{0} \notin C_{n} \cup \partial_{\infty} C_{n}$. Note that for every $n \in \mathbb{N}-\{0\}$, the map $f_{n}$ is a $\kappa$-penetration map in $C_{n}$. As $h \geq c_{1}^{\prime \prime}$, there exists a geodesic line $\gamma$ starting from $\xi$ and entering $C_{0}$ at time 0 , such that $\mathfrak{p h}_{C_{0}}(\gamma)=h$ and $\ell_{C_{n}}(\gamma) \leq c_{1}^{\prime \prime}$ for every $n \in \mathbb{N}-\{0\}$ such that $\gamma$ meets $C_{n}$ at a positive time.

Let $\xi^{\prime}$ be the other endpoint of $\gamma$. This point is not in $\partial_{\infty} C_{0}$. Applying Theorem 5.1 again, as above except that now $\xi_{0}=\xi^{\prime}$, we get that there exists a geodesic line $\gamma^{\prime}$ starting from $\xi^{\prime}$ and entering $C_{0}$ at time 0 , such that $\mathfrak{p h}_{C_{0}}\left(\gamma^{\prime}\right)=h$ and $\ell_{C_{n}}\left(\gamma^{\prime}\right) \leq c_{1}^{\prime \prime}$ for every $n \in \mathbb{N}-\{0\}$ such that $\gamma^{\prime}$ meets $C_{n}$ at a positive time.

Assume by contradiction that there exists $n \in \mathbb{N}-\{0\}$ such that $\mathfrak{p h}_{C_{n}}\left(\gamma^{\prime}\right)>c_{2}^{\prime \prime}>0$. Then $\gamma^{\prime}$ enters $C_{n}$ at the point $x_{n}^{\prime}$, exiting it at the point $y_{n}^{\prime}$ at a nonpositive time, as $c_{2}^{\prime \prime}>c_{1}^{\prime \prime}$. In particular, if $x^{\prime}=\gamma^{\prime}(0)$ is the entering point of $\gamma^{\prime}$ in $C_{0}$, then $x^{\prime}, y_{n}^{\prime}, x_{n}^{\prime}, \xi^{\prime}$ are in this order on $\gamma^{\prime}$ (see the picture in the proof of Corollary 4.11).

Let $y$ be the exiting point of $\gamma$ out of $H_{0}$. With $c_{1}=1 / 19$, as in the proof of Lemma 4.6, since $\mathfrak{p h}_{C_{0}}(\gamma)$ and $\mathfrak{p h}_{C_{0}}\left(\gamma^{\prime}\right)$ are equal to

$$
h \geq c_{1}^{\prime \prime} \geq h_{1}^{\prime}\left(\infty, 0, h_{0}\left(\infty, 0, c_{1}^{\prime}(\infty)\right)\right) \geq h_{0}\left(\infty, 0, c_{1}^{\prime}(\infty)\right)=h^{\prime}\left(\infty, \sinh c_{1}\right)
$$

by the definition of $c_{1}^{\prime \prime}, h_{1}^{\prime}, h_{0}$, we have $d\left(x^{\prime}, y\right) \leq c_{1}$.

Let $\xi_{n}$ be the point at infinity of $H_{n}$. Let $p^{\prime}$ be the point in $\left[x_{n}^{\prime}, y_{n}^{\prime}\right]$ the closest to $\xi_{n}$, so that

$$
d\left(p^{\prime}, y_{n}^{\prime}\right) \geq \beta_{\xi_{n}}\left(y_{n}^{\prime}, p^{\prime}\right)=\mathfrak{p h}_{C_{n}}\left(\gamma^{\prime}\right) / 2>c_{2}^{\prime \prime} / 2
$$


Let $p$ be the point of $\gamma$ the closest to $p^{\prime}$. By convexity and the definition of $c_{2}^{\prime \prime}$, we have

$$
d\left(p^{\prime}, p\right)=d\left(p^{\prime}, \gamma\right) \leq d\left(x^{\prime}, \gamma\right) \leq d\left(x^{\prime}, y\right) \leq c_{1}<c_{2}^{\prime \prime} / 2 .
$$

Hence $p$ belongs to the interior of $C_{n}$. If $\left.p \in\right] \xi, y$ ], then the closest point to $x^{\prime}$ on $\gamma$ lies in $] \xi, p[$ and by convexity,

$$
c_{2}^{\prime \prime} / 2<d\left(p^{\prime}, y_{n}^{\prime}\right) \leq d\left(p^{\prime}, x^{\prime}\right) \leq d\left(p^{\prime}, p\right)+d\left(p, x^{\prime}\right) \leq d\left(p^{\prime}, p\right)+d\left(y, x^{\prime}\right) \leq 2 c_{1},
$$

a contradiction, as by the definition of $c_{2}^{\prime \prime}$, of $c_{1}^{\prime \prime}$ and of $h^{\prime}(\epsilon, \eta)$ (see Equations (5) and (11)), we have

$$
c_{2}^{\prime \prime} \geq c_{1}^{\prime \prime}+2 c_{1} \geq h^{\prime}\left(\epsilon, \sinh c_{1}\right)+2 c_{1} \geq 2 \sinh c_{1}+2 c_{1}>4 c_{1} .
$$

Hence $p \in] y, \xi^{\prime}\left[\subset \gamma(] 0,+\infty[)\right.$, so that $\gamma$ meets $C_{n}$ at a positive time. But, by Lemma 3.3 and the definition of $c_{2}^{\prime \prime}$,

$$
\begin{aligned}
\ell_{C_{n}}(\gamma) & \geq \mathfrak{p h}_{C_{n}}(\gamma)-c_{1}^{\prime}(\infty) \geq 2 \beta_{\xi_{n}}\left(y_{n}^{\prime}, p\right)-c_{1}^{\prime}(\infty) \\
& \geq 2\left(\beta_{\xi_{n}}\left(y_{n}^{\prime}, p^{\prime}\right)-d\left(p, p^{\prime}\right)\right)-c_{1}^{\prime}(\infty)>2\left(c_{2}^{\prime \prime} / 2-c_{1}\right)-c_{1}^{\prime}(\infty)=c_{1}^{\prime \prime} .
\end{aligned}
$$

This contradicts the construction of $\gamma$.

Let $M$ be a complete nonelementary geometrically finite Riemannian manifold with sectional curvature at most -1 (see for instance Bowditch [10] for a general reference). Recall that a cusp of $M$ is an asymptotic class of minimizing geodesic rays in $M$ along which the injectivity radius converges to 0 . If $M$ has finite volume, then the set of cusps of $M$ is in bijection with the (finite) set of ends of $M$, by the map which associates to a representative of a cusp the end of $M$ towards which it converges. Let $\pi: \widetilde{M} \rightarrow M$ be a universal Riemannian covering of $M$, with covering group $\Gamma$. If $e$ is a cusp of $M$, and $\rho_{e}$ a minimizing geodesic ray in the class $e$, as $M$ is geometrically finite and nonelementary, there exists a horoball $H_{e}$ in $\widetilde{M}$ centered at the point at infinity $\xi_{e}$ of a fixed lift $\widetilde{\rho_{e}}$ of $\rho_{e}$ in $\widetilde{M}$, such that $\gamma H_{e}$ and $H_{e}$ have disjoint interiors if $\gamma \in \Gamma$ does not fix $\xi_{e}$ (see for instance Buser and Karcher [12], Bowditch [10] and Hersonsky and Paulin [32]). This horoball is unique if maximal (for the inclusion). The image $V_{e}$ of $H_{e}$ in $M$ is called a Margulis neighbourhood of $e$, and the maximal Margulis neighbourhood of $e$ if $H_{e}$ is maximal. We assume that $\widetilde{\rho_{e}}$ starts from the boundary of $H_{e}$. Let $\mathrm{ht}_{e}: M \rightarrow \mathbb{R}$ be the map defined by

$$
\mathrm{ht}_{e}(x)=\lim _{t \rightarrow \infty}\left(t-d\left(\rho_{e}(t), x\right)\right),
$$

called the height function with respect to $e$. Note that if $V_{e}^{\prime} \subset V_{e}$ is another Margulis neighbourhood of $e$, then the height function associated to the choice of $V_{e}^{\prime}$ becomes 
ht $_{e}-\tau$ for some nonnegative constant $\tau$. Let $\operatorname{maxht}_{e}: T^{1} M \rightarrow \mathbb{R}$ be defined by

$$
\operatorname{maxht}_{e}(\gamma)=\sup _{t \in \mathbb{R}} \mathrm{ht}_{e}(\gamma(t)) \text {. }
$$

The maximum height spectrum of the pair $(M, e)$ is the subset of $]-\infty,+\infty]$ defined by

$$
\operatorname{MaxSp}(M, e)=\operatorname{maxht}_{e}\left(T^{1} M\right) .
$$

Corollary 5.4 Let $M$ be a complete, nonelementary geometrically finite Riemannian manifold with sectional curvature at most -1 and dimension at least 3 , and let $e$ be a cusp of $M$. Then $\operatorname{Max} \operatorname{Sp}(M, e)$ contains $\left[c_{2}^{\prime \prime}(\infty) / 2,+\infty\right]$.

Note that $c_{2}^{\prime \prime}(\infty) / 2 \approx 4.1856$, hence Theorem 1.2 of the introduction follows.

Proof With the above notation, let $\left(H_{n}\right)_{n \in \mathbb{N}}$ be the $\Gamma$-equivariant family of horoballs in $\widetilde{M}$ with pairwise disjoint interiors such that $H_{0}=H_{e}$. Apply Corollary 5.3 to this family to get, for every $h \geq c_{2}^{\prime \prime}(\infty) \geq c_{1}^{\prime \prime}(\infty, 0,0)$, a geodesic line $\widetilde{\gamma}$ in $\widetilde{M}$ with $\mathfrak{p h}_{H_{0}}(\tilde{\gamma})=h$ and $\mathfrak{p h}_{H_{n}}(\tilde{\gamma}) \leq c_{2}^{\prime \prime}(\infty)$ for every $n \geq 1$. Let $\gamma$ be the locally geodesic line in $M$ image by $\pi$ of $\tilde{\gamma}$. Observe that $\mathrm{ht}_{e} \circ \pi=\beta_{H_{n}}$ in $H_{n}$ and that $\mathfrak{p h}_{H_{n}}(\tilde{\gamma})=2 \sup _{t \in \mathbb{R}} \beta_{H_{n}}(\tilde{\gamma}(t))$ (see Section 3.1). Hence $\sup _{t \in \mathbb{R}} \mathrm{ht}_{e}(\gamma(t))=h / 2$ and the result follows.

Schmidt and Sheingorn [55] treated the case of two-dimensional manifolds of constant curvature -1 (hyperbolic surfaces) with a cusp. They showed that in that case $\operatorname{Max} \operatorname{Sp}(M, e)$ contains the interval $[\log 100,+\infty] \approx[4.61,+\infty]$. This paper [55] was a starting point of our investigations, although the method we use is quite different from theirs.

Let $\mathcal{P}$ be a (necessarily finite) nonempty set of cusps of $M$. For every $e$ in $\mathcal{P}$, choose a maximal horoball $H_{e}$, with point at infinity $\xi_{e}$ as above Corollary 5.4. The horoballs of the family $\left(g H_{e}\right)_{g \in \Gamma / \Gamma_{\xi_{e}}, e \in \mathcal{P}}$ may have intersecting interiors. But as $M$ is geometrically finite and nonelementary, there exists $t \geq 0$ such that two distinct elements in $\left(g H_{e}[t]\right)_{g \in \Gamma / \Gamma_{\xi_{e}}, e \in \mathcal{P}}$ have disjoint interior; see for instance Buser and Karcher [12] and Bowditch [10]. Let $t_{\mathcal{P}}$ be the lower bound of all such $t$ 's. For every $\gamma \in T^{1} M$, define

$$
\operatorname{maxht}_{\mathcal{P}}(\gamma)=\max _{e \in \mathcal{P}} \operatorname{maxht}_{e}(\gamma) \quad \text { and } \quad \operatorname{MaxSp}(M, \mathcal{P})=\operatorname{maxht}_{\mathcal{P}}\left(T^{1} M\right) .
$$

Remark Let $\mathcal{C}$ be the set of all cusps of $M$. Under the same hypotheses as in Corollary 5.4, the following two assertions hold, by applying Corollary 5.3 to the family of horoballs $\left(g H_{e^{\prime}}^{\prime}\right)_{g \in \Gamma / \Gamma_{e^{\prime}}, e^{\prime} \in \mathcal{C}}$ with $H_{e^{\prime}}^{\prime}=H_{e}$ if $e^{\prime}=e$, and $H_{e^{\prime}}^{\prime}=H_{e^{\prime}}[t]$ for some $t$ 
big enough otherwise, for the first assertion, and to the family $\left(g H_{e}\left[t_{\mathcal{P}}\right]\right)_{g \in \Gamma / \Gamma_{\xi_{e}}, e \in \mathcal{P}}$ for the second one.

(1) For every cusp $e$ of $M$, there exists a constant $t \geq 0$ such that for every $h \geq t$, there exists a locally geodesic line $\gamma$ in $M$ such that $\operatorname{maxht}_{e}(\gamma)=h$ and $\operatorname{maxht}_{e^{\prime}}(\gamma) \leq t$ for every cusp $e^{\prime} \neq e$ in $M$.

(2) Let $\mathcal{P}$ be a nonempty set of cusps of $M$. Then $\operatorname{Max} \operatorname{Sp}(M, \mathcal{P})$ contains the halfline $\left[c_{2}^{\prime \prime}(\infty) / 2+t_{\mathcal{P}},+\infty\right]$.

Now, we prove the analogs of Corollaries 5.3 and 5.4 for families of balls with disjoint interiors. Let

$$
R_{0}^{\min }=7 \sinh c_{1}^{\prime}(\infty)+\frac{3}{2} c_{1}^{\prime}(\infty) \approx 22.4431 .
$$

Corollary 5.5 Let $X$ be a complete simply connected Riemannian manifold with sectional curvature at most -1 and dimension at least 3 , and let $\left(B_{n}\right)_{n \in \mathbb{N}}$ be a family of balls in $X$ with disjoint interiors such that the radius $R_{0}$ of $B_{0}$ is at least $R_{0}^{\mathrm{min}}$. For every $h \in\left[c_{1}^{\prime \prime}\left(R_{0}^{\min }, 0,0\right), 2 R_{0}-2 c_{1}^{\prime}\left(R_{0}^{\min }\right)\right]$, there exists a geodesic line $\gamma$ in $X$ with $\mathfrak{p h}_{B_{0}}(\gamma)=h$ and $\mathfrak{p h}_{B_{n}}(\gamma) \leq c_{2}^{\prime \prime}\left(R_{0}^{\min }\right)$ for all $n \geq 1$.

Proof We start by some computations. Let $\epsilon>0$. With $c_{1}=c_{1}^{\prime}(\epsilon)$ and $c_{5}=c_{5}(\epsilon, 0)$ as in Section 4.2, we have $c_{5} \geq 6 \sinh c_{1}$ since $c_{3}^{\prime}(\epsilon) \geq 3$ by the definition of $c_{3}^{\prime}(\epsilon)$ in Equation (6). By the definition of $h_{0}$ in Section 4.2 and of $h^{\prime}$ in Equation (5), we have $h_{0}\left(\epsilon, 0, c_{1}^{\prime}(\infty)\right) \geq h^{\prime}\left(\epsilon, \sinh c_{1}\right) \geq 2 \sinh c_{1}$. Hence, by the definitions of $c_{2}^{\prime \prime}(\epsilon), c_{1}^{\prime \prime}(\epsilon, 0,0), h_{1}^{\prime}$ and as $\epsilon \mapsto c_{1}^{\prime}(\epsilon)$ is decreasing,

$$
\begin{aligned}
c_{2}^{\prime \prime}(\epsilon) & =c_{1}^{\prime \prime}(\epsilon, 0,0)+c_{1}^{\prime}(\infty)+2 c_{1} \\
& =\max \left\{2 c_{1}, h_{0}\left(\epsilon, 0, c_{1}^{\prime}(\infty)\right)+2 c_{5}\right\}+c_{1}^{\prime}(\infty)+2 c_{1} \\
& \geq 2 \sinh c_{1}+12 \sinh c_{1}+c_{1}^{\prime}(\infty)+2 c_{1} \geq 14 \sinh c_{1}^{\prime}(\infty)+3 c_{1}^{\prime}(\infty) .
\end{aligned}
$$

Define now $\epsilon=R_{0}^{\min }$, so that $2 \epsilon \leq c_{2}^{\prime \prime}(\epsilon)$ and $R_{0} \geq \epsilon$. For every $n \neq 0$, let $R_{n}$ be the radius of the ball $B_{n}$. If for some $n \neq 0$ we have $2 R_{n} \leq c_{2}^{\prime \prime}(\epsilon)$, then $\mathfrak{p h}_{B_{n}}(\gamma) \leq c_{2}^{\prime \prime}(\epsilon)$ and the last assertion of Corollary 5.5 holds for this $n$. Hence up to removing balls, we may assume that $R_{n} \geq c_{2}^{\prime \prime}(\epsilon) / 2 \geq \epsilon$ for every $n \neq 0$, so that the balls in $\left(B_{n}\right)_{n \in \mathbb{N}}$ are $\epsilon$-convex.

The end of the proof is now exactly as the proof of Corollary 5.3, with the following modifications: $\epsilon=R_{0}^{\min } ; c_{1}^{\prime \prime}=c_{1}^{\prime \prime}(\epsilon, 0,0) ; c_{2}^{\prime \prime}=c_{2}^{\prime \prime}(\epsilon) ; \xi$ is any point in $\partial_{\infty} X$; $C_{n}=B_{n}$ for every $n$ in $\mathbb{N}$; we apply Theorem 5.2 instead of Theorem 5.1, which is possible by the range assumption on $h$; we take now $c_{1}=c_{1}^{\prime}(\epsilon)$, so that we still have $d\left(x^{\prime}, y\right) \leq c_{1}$ by Lemma 2.3; $\xi_{n}$ is now the center of $B_{n}$, and $\beta_{\xi_{n}}(u, v)=$ $d\left(u, \xi_{n}\right)-d\left(v, \xi_{n}\right)$ (see Section 2.1). Besides that, the proof is unchanged. 
A heavy computation shows that

$$
c_{1}^{\prime}\left(R_{0}^{\min }\right) \approx 1.7627, c_{1}^{\prime \prime}\left(R_{0}^{\min }, 0,0\right) \approx 101.4169 \quad \text { and } \quad c_{2}^{\prime \prime}\left(R_{0}^{\min }\right) \approx 106.7051 .
$$

Note that the above corollary is nonempty only if $R_{0} \geq c_{1}^{\prime \prime}\left(R_{0}^{\min }, 0,0\right) / 2+c_{1}^{\prime}\left(R_{0}^{\min }\right) \approx$ 52.4712. The constants in the following corollary are not optimal. Theorem 1.3 in the introduction follows from it.

Corollary 5.6 Let $M$ be a complete Riemannian manifold with sectional curvature at most -1 and dimension at least 3 , let $\left(x_{i}\right)_{i \in I}$ be a finite or countable family of points in $M$ with $r_{i}=\operatorname{inj}_{M} x_{i}$, such that $d\left(x_{i}, x_{j}\right) \geq r_{i}+r_{j}$ if $i \neq j$ and such that $r_{i_{0}} \geq 56$ for some $i_{0} \in I$. Then, for every $d \in\left[2, r_{i_{0}}-54\right]$, there exists a locally geodesic line $\gamma$ passing at distance exactly $d$ from $x_{i_{0}}$ at time 0 , remaining at distance greater than $d$ from $x_{i_{0}}$ at any nonzero time, and at distance at least $r_{i}-56$ from $x_{i}$ for every $i \neq i_{0}$. In particular,

$$
\min _{t \in \mathbb{R}} d\left(\gamma(t), x_{i_{0}}\right)=d
$$

Proof Let $\pi: \widetilde{M} \rightarrow M$ be a universal covering of $M$, with covering group $\Gamma$, and fix a lift $\tilde{x}_{i}$ of $x_{i}$ for every $i \in I$. Let $B_{i}$ be the ball $B \widetilde{M}\left(\tilde{x}_{i}, r_{i}\right)$. Apply Corollary 5.5 to the family of balls $\left(g B_{i}\right)_{g \in \Gamma, i \in I}$ in $X=\widetilde{M}$, which have pairwise disjoint interiors by the definition of $r_{i}$ and the assumption on $d\left(x_{i}, x_{j}\right)$. Note that $r_{i_{0}} \geq 56 \geq R_{0}^{\min }$ (see the definition of $\left.R_{0}^{\min }\right)$. Let $h=2\left(r_{i_{0}}-d\right)$, which belongs to [108, $\left.2 r_{i_{0}}-4\right]$, which is contained in $\left[c_{1}^{\prime \prime}\left(R_{0}^{\min }, 0,0\right), 2 r_{i_{0}}-2 c_{1}^{\prime}\left(R_{0}^{\min }\right)\right]$ by the previous computations. Then Corollary 5.5 implies that there exists a geodesic line $\tilde{\gamma}$ in $\widetilde{M}$ such that $\mathfrak{p h}_{B_{i_{0}}}(\tilde{\gamma})=h$ and $\mathfrak{p h}_{g B_{i}}(\tilde{\gamma}) \leq c_{2}^{\prime \prime}\left(R_{0}^{\min }\right)<108$ for all $(g, i) \neq\left(1, i_{0}\right)$. Parametrize $\tilde{\gamma}$ such that its closest point to $\tilde{x}_{i_{0}}$ is at time $t=0$. Let $\gamma=\pi \circ \tilde{\gamma}$, then the result follows by the definition of $\mathfrak{p h}_{C}$ (see Section 3.1).

\subsection{Spiralling around totally geodesic subspaces}

In this subsection, we apply Proposition 4.10 and Corollary 4.11 when $C_{0}$ is a tubular neighbourhood of a totally geodesic submanifold. We only give a few of the various possible applications, others can be obtained by varying the objects $\left(C_{n}\right)_{n \in \mathbb{N}-\{0\}}$, as well as the various subcases in Proposition 3.7 (3) and (4).

Theorem 5.7 Let $\epsilon>0, \delta \geq 0$. Let $X$ be a complete simply connected Riemannian manifold with sectional curvature at most -1 and dimension at least 3 . Let $L$ be a complete totally geodesic submanifold of $X$ with dimension at least 2, different from $X$, and $C_{0}=\mathcal{N}_{\epsilon} L$. Let $\left(C_{n}\right)_{n \in \mathbb{N}-\{0\}}$ be a family of $\epsilon$-convex subsets in $X$ 
such that $\operatorname{diam}\left(C_{n} \cap C_{m}\right) \leq \delta$ for all $n \neq m$ in $\mathbb{N}$. Let either $f_{0}=\mathfrak{f t p}_{L}$ or $f_{0}=\ell_{\mathcal{N}_{\epsilon} L}$, with $X$ having constant curvature in this second case. Let

$$
h_{0}^{\prime}=h_{0}\left(\epsilon, \delta, \max \left\{\left\|f_{0}-\ell_{\mathcal{N}_{\epsilon} L}\right\|_{\infty}, 2\left\|f_{0}-\mathfrak{f t p}_{L}\right\|_{\infty}+2 \epsilon-8 c_{1}^{\prime}(\epsilon)\right\}\right)
$$

and $h \geq h_{1}^{\prime}=h_{1}^{\prime}\left(\epsilon, \delta, h_{0}^{\prime}\right)$.

- For every $\xi \in\left(X \cup \partial_{\infty} X\right)-\left(C_{0} \cup \partial_{\infty} C_{0}\right)$, there exists a geodesic ray or line $\gamma$ starting from $\xi$ and entering $\mathcal{N}_{\epsilon} L$ at time 0 with $f_{0}(\gamma)=h$, and with $\ell_{C_{n}}(\gamma) \leq h_{1}^{\prime}$ for every $n \neq 0$ such that $\gamma(] \delta,+\infty[)$ meets $C_{n}$.

- There exists a geodesic line $\gamma$ in $X$ with $f_{0}(\gamma)=h$, and with

$$
\ell_{C_{n}}(\gamma) \leq h_{1}^{\prime}+c_{3}^{\prime}(\epsilon)\left(\delta+c_{1}^{\prime}(\epsilon)\right)+c_{1}^{\prime}(\epsilon)
$$

for all $n \neq 0$.

Note that if $\ell_{C_{n}}(\gamma) \leq c$, then $f(\gamma) \leq c+\kappa$ for any $\kappa$-penetration map $f$ in $C_{n}$.

Proof We apply Proposition 4.10 and Corollary 4.11 with $\xi_{0}=\xi, \epsilon_{0}=\epsilon, \delta_{0}=\delta$,

$$
\kappa_{0}=\max \left\{\left\|f_{0}-\ell_{\mathcal{N}_{\epsilon} L}\right\|_{\infty}, 2\left\|f_{0}-\mathfrak{f} \mathfrak{t p}_{L}\right\|_{\infty}+2 \epsilon-8 c_{1}^{\prime}(\epsilon)\right\},
$$

and $h_{0}^{\prime}=h_{0}\left(\epsilon_{0}, \delta_{0}, \kappa_{0}\right)$. In particular, $f_{0}$ is a continuous $\kappa_{0}$-penetration map in $C_{0}$. As $L$ is a complete totally geodesic submanifold of dimension and codimension at least 1 , there does exist a geodesic line $\gamma_{0}$ in $X$ starting from $\xi_{0}$ such that $f_{0}\left(\gamma_{0}\right)=h$. Let $h_{0}^{\min }=\delta_{0}$ and $h^{\min }=4 c_{1}^{\prime}(\epsilon)+2 \epsilon+\delta+2\left\|f_{0}-\mathfrak{f t p}\right\|_{L} \|_{\infty}$. By the definitions of $h_{1}^{\prime}(\cdot, \cdot, \cdot), h_{0}(\cdot, \cdot, \cdot), c_{5}(\cdot, \cdot)$ in Section 4.2, we have

$$
h_{0}^{\prime}=h_{0}\left(\epsilon_{0}, \delta_{0}, \kappa_{0}\right)>\delta_{0}=h_{0}^{\min },
$$

and

$$
\begin{aligned}
h & \geq h_{1}^{\prime}=h_{0}\left(\epsilon_{0}, \delta_{0}, \kappa_{0}\right)+2 c_{5}\left(\epsilon_{0}, \delta_{0}\right) \geq \kappa_{0}+12 \sinh \left(c_{1}^{\prime}\left(\epsilon_{0}\right)+\delta_{0}\right) \\
& \geq \kappa_{0}+12 c_{1}^{\prime}(\epsilon)+\delta \geq h^{\min } .
\end{aligned}
$$

The family $\left(C_{n}\right)_{n \in \mathbb{N}}$ hence satisfies the local prescription property (iv) by Proposition 3.7 (3). Therefore, the result follows from Proposition 4.10 and Corollary 4.11

Remark 5.8 If the $C_{n}$ 's are disjoint from $\mathcal{N}_{\epsilon} L$ (and $\delta=0$ ), then the same result as Theorem 5.7 also holds when $L$ has dimension 1, by replacing Proposition 3.7 (3) by Proposition 3.7 (4) in the above proof and $h_{0}^{\min }=\delta_{0}$ by $h_{0}^{\min }=0$.

Theorem 5.9 Let $\epsilon>0, \delta \geq 0$. Let $X$ be a complete simply connected Riemannian manifold with sectional curvature at most -1 and dimension at least 3 . Let $\left(L_{n}\right)_{n \in \mathbb{N}}$ be a family of geodesic lines in $X$, such that $\operatorname{diam}\left(\mathcal{N}_{\epsilon} L_{n} \cap \mathcal{N}_{\epsilon} L_{m}\right) \leq \delta$ for all $n \neq m$ in $\mathbb{N}$. Let either $f_{0}=\mathfrak{f t p}_{L_{0}}$, or $f_{0}=\ell_{\mathcal{N}_{\epsilon} L_{0}}$ if $X$ has constant curvature, or $f_{0}=\mathfrak{c r p}_{L_{0}}$ 
if the metric spheres for the Hamenstädt distances (on $\partial_{\infty} X-\{\xi\}$ for any $\xi \in \partial_{\infty} X$ ) are topological spheres. Let

$$
\begin{aligned}
h_{0}^{\prime}=\max \left\{5 c_{1}^{\prime}(\epsilon)+\right. & 5 \epsilon+\delta, \\
& \left.h_{0}\left(\epsilon, \delta, \max \left\{\left\|f_{0}-\ell_{\mathcal{N}_{\epsilon} L_{0}}\right\|_{\infty},\left\|f_{0}-\mathfrak{f t p}_{L_{0}}\right\|_{\infty}+2 \epsilon-8 c_{1}^{\prime}(\epsilon)\right\}\right)\right\}
\end{aligned}
$$

and $h \geq h_{1}^{\prime}=h_{1}^{\prime}\left(\epsilon, \delta, h_{0}^{\prime}\right)$.

- For every $\xi \in\left(X \cup \partial_{\infty} X\right)-\left(\mathcal{N}_{\epsilon} L_{0} \cup \partial_{\infty} L_{0}\right)$ (and $\xi \in \partial_{\infty} X-\partial_{\infty} L_{0}$ if $\left.f_{0}=\mathfrak{c r p}_{L_{0}}\right)$, there exists a geodesic ray or line $\gamma$ starting from $\xi$ and entering $\mathcal{N}_{\epsilon} L_{0}$ at time 0 with $f_{0}(\gamma)=h$, such that $\ell_{\mathcal{N}_{\epsilon} L_{n}}(\gamma) \leq h_{1}^{\prime}$ for every $n \neq 0$ such that $\gamma(] \delta,+\infty[)$ meets $\mathcal{N}_{\epsilon} L_{n}$.

- There exists a geodesic line $\gamma$ in $X$ such that $f_{0}(\gamma)=h$, and, if $n \neq 0$, then $\ell_{\mathcal{N}_{\epsilon} L_{n}}(\gamma) \leq h_{1}^{\prime}+c_{3}^{\prime}(\epsilon)\left(\delta+c_{1}^{\prime}(\epsilon)\right)+c_{1}^{\prime}(\epsilon)$.

Note that if $\ell_{\mathcal{N}_{\epsilon} L_{n}}(\gamma) \leq c$, then $f(\gamma) \leq c+\kappa$ for any $\kappa$-penetration map $f$ in $\mathcal{N}_{\epsilon} L_{n}$.

Proof As in the previous proof, we apply Proposition 4.10 and Corollary 4.11 with $C_{n}=\mathcal{N}_{\epsilon} L_{n}, \epsilon_{0}=\epsilon, \delta_{0}=\delta, \xi_{0}=\xi$,

$$
\kappa_{0}=\max \left\{\left\|f_{0}-\ell_{\mathcal{N}_{\epsilon} L_{0}}\right\|_{\infty},\left\|f_{0}-\mathfrak{f t p} L_{L_{0}}\right\|_{\infty}+2 \epsilon-8 c_{1}^{\prime}(\epsilon)\right\} .
$$

For every $n \neq 0$, let $h_{0}^{\min }=3 c_{1}^{\prime}(\epsilon)+3 \epsilon+\delta+\left\|\ell_{\mathcal{N}_{\epsilon} L_{n}}-\mathfrak{f t p}_{L_{n}}\right\|_{\infty}$ and $h^{\mathrm{min}}=$ $4 c_{1}^{\prime}(\epsilon)+2 \epsilon+\delta+\left\|f_{0}-\mathfrak{f t p}_{L}\right\|_{\infty}$. In particular, $h_{0}^{\prime} \geq 5 c_{1}^{\prime}(\epsilon)+5 \epsilon+\delta \geq h_{0}^{\min }$ by Lemma 3.4. As in the end of the previous proof, the family $\left(C_{n}\right)_{n \in \mathbb{N}}$ hence satisfies the property (iv) by Proposition 3.7 (4), and the result follows.

Let $M$ be a complete Riemannian manifold with sectional curvature at most -1 and dimension $n \geq 3$. Fix a universal cover $\widetilde{M} \rightarrow M$ of $M$. For $\epsilon>0, \delta \geq 0$, a (possibly not connected, but such that any two components have equal dimension) immersed complete totally geodesic submanifold $L$ (of dimension at least 1 and at most $n-1)$ will be called $(\epsilon, \delta)$-separated if the diameter of the intersection of the $\epsilon$-neighbourhoods of two lifts to $\widetilde{M}$ of two components of $L$ is at most $\delta$.

Examples (1) If $L$ is compact and embedded, then there exists $\epsilon>0$ such that $L$ is $(\epsilon, 0)$-separated. For instance, a finite union of disjoint simple closed geodesics is $(\epsilon, 0)$-separated for $\epsilon$ small enough.

(2) If $L$ is compact, and if $L$ is self-transverse (that is, if the tangent spaces at every double point of $L$ are transverse), then for every $\epsilon>0$ small enough, $L$ is $(\epsilon, 1)$-separated. In particular, a finite union of closed geodesics (possibly nonsimple) is $(\epsilon, 1)$-separated for $\epsilon$ small enough. 
(3) The lift of a locally geodesic line $\gamma: \mathbb{R} \rightarrow M$ to the unit tangent bundle $T^{1} M$ is the map $\tilde{\gamma}: \mathbb{R} \rightarrow T^{1} M$ (or by abuse its image) given by $\tilde{\gamma}(t)=\left(\gamma(t), \gamma^{\prime}(t)\right)$ for every $t \in \mathbb{R}$. For every $\rho>0$, if the $\rho$-neighbourhood (for the standard Riemannian metric of $\left.T^{1} M\right)$ of the lift of $\gamma$ to $T^{1} M$ is a tubular neighbourhood, then there exists $\delta(\rho) \geq 0$ such that $\gamma$ is $(\rho, \delta(\rho))$-separated. Indeed, if the intersection of the $\rho$-neighbourhoods of two different lifts to a universal cover of $\gamma$ has diameter big enough (depending only on $\rho$ ), then by arguments similar to the ones in the proof of Lemma 2.2, two subsegments of the two lifts will follow themselves closely for some time, hence the tangent vectors at two points on these two lifts will be closer than $\rho$.

Let $L$ be an $(\epsilon, \delta)$-separated immersed complete totally geodesic submanifold. Let $\left(\widetilde{L}_{\alpha}\right)_{\alpha \in \mathcal{A}}$ be the family of (connected) complete totally geodesic submanifolds of $\widetilde{M}$, that are the lifts to $\widetilde{M}$ of the components of $L$. Note that in particular, the family $\left(\mathcal{N}_{\epsilon}\left(\widetilde{L}_{\alpha}\right)\right)_{\alpha \in \mathcal{A}}$ is locally finite.

Let $f$ be one of the symbols $\ell, \mathfrak{b p}, \mathfrak{f t p}, \mathfrak{c r p}$ and assume that $L$ has dimension 1 if $f=\mathfrak{c r p}$. Let $\kappa_{f}$ be respectively $0,2 c_{1}^{\prime}(\epsilon), 2 c_{1}^{\prime}(\epsilon)+2 \epsilon, 2 c_{1}^{\prime}(\epsilon)+2 c_{1}^{\prime}(\infty)+2 \epsilon$. For every $\alpha \in \mathcal{A}$, let $f_{\alpha}=\ell_{\mathcal{N}_{\epsilon} L_{\alpha}}, \mathfrak{b p}_{L_{\alpha}}, \mathfrak{f t p}_{L_{\alpha}}, \mathfrak{c t p}_{L_{\alpha}}$ respectively, which is a $\kappa_{f}$ penetration map in $\mathcal{N}_{\epsilon} L_{\alpha}$ by Section 3.1. For every locally geodesic line $\gamma$ in $M$, consider a lift $\tilde{\gamma}$ of $\gamma$ to $\widetilde{M}$.

The family $\left(f_{\alpha}(\tilde{\gamma})\right)_{\alpha \in \mathcal{A}}$ will be called the family of spiraling times of $\gamma$ along $L$ with respect to $f$ (and length spiraling times, fellow-traveling times or crossratio spiraling times if $f=\ell, \mathfrak{f t p}, \mathfrak{c r p}$ respectively). Up to permutation of $\mathcal{A}$, it does not depend on the choice of the lift $\tilde{\gamma}$ of $\gamma$. The entering times of $\tilde{\gamma}$ in the sets $\mathcal{N}_{\epsilon} L_{\alpha}$ with $f_{\alpha}(\tilde{\gamma})>\delta+\kappa_{f}$, where $\alpha$ varies in $\mathcal{A}$, form a discrete subset (with multiplicity one) of $\mathbb{R}$, as $\mathcal{N}_{\epsilon} L_{\alpha} \cap \mathcal{N}_{\epsilon} L_{\beta}$ has diameter at most $\delta$ if $\alpha \neq \beta$. We will only be interested in the corresponding spiraling times. It is also then possible to order these spiraling times using the order given by the parametrisation on $\tilde{\gamma}$, but we will not need this here.

Corollary 5.10 Let $M$ be a complete Riemannian manifold with sectional curvature at most -1 and dimension $n \geq 3$. Let $\epsilon>0, \delta \geq 0$. Let $L$ be an $(\epsilon, \delta)$-separated immersed complete totally geodesic submanifold (of dimension at least 1 and at most $n-1)$. Let $f$ be one of the symbols $\ell, \mathfrak{f t p}, \mathfrak{c r p}$, and $\kappa_{f}^{\prime}=\max \left\{0,6 \epsilon-4 c_{1}^{\prime}(\epsilon)\right\}$, $2 c_{1}^{\prime}(\epsilon)+2 \epsilon, 2 c_{1}^{\prime}(\epsilon)+2 \epsilon+2 c_{1}^{\prime}(\infty)$ respectively. If $f=\ell$, assume that $M$ has constant curvature. If $f=\mathfrak{c r p}$, assume that $L$ has dimension 1 and that the metric spheres for the Hamenstädt distances (on the punctured boundary of a universal cover of $M$ ) are topological spheres.

For every

$$
h \geq h_{1}^{\prime}=h_{1}^{\prime}\left(\epsilon, \delta, \max \left\{5 c_{1}^{\prime}(\epsilon)+5 \epsilon+\delta, h_{0}\left(\epsilon, \delta, \kappa_{f}^{\prime}\right)\right\}\right),
$$


there exists a locally geodesic line $\gamma$ in $M$ having one spiraling time with respect to $f$ exactly $h$, and all others being at most $h_{1}^{\prime}+c_{3}^{\prime}(\epsilon)\left(\delta+c_{1}^{\prime}(\epsilon)\right)+c_{1}^{\prime}(\epsilon)$.

If furthermore $M$ is nonelementary and geometrically finite, then for every cusp $e$ of $M$, we may also assume that the locally geodesic line $\gamma$ does not enter too much into the maximal Margulis neighbourhood of $e$; more precisely we can assume that $\gamma$ satisfies

$$
\operatorname{maxht}_{e}(\gamma) \leq \sup _{x \in L} \mathrm{ht}_{e}(x)+\epsilon+\frac{1}{2}\left(h_{1}^{\prime}+c_{3}^{\prime}(\epsilon)\left(\delta+c_{1}^{\prime}(\epsilon)\right)+c_{1}^{\prime}(\epsilon)\right) .
$$

Proof Let $\pi: \widetilde{M} \rightarrow M$ be a universal cover of $M$, with covering group $\Gamma$. With $\kappa_{0}$ the constant in the proofs of Theorem 5.7 if the dimension of $L$ is at least 2, and Theorem 5.9 otherwise, it is easy to check, using Section 3.1, that $\kappa_{f}^{\prime} \geq \kappa_{0}$ for every case of $f$. Note that $h_{0}(\cdot, \cdot, \cdot)$ and $h_{1}^{\prime}(\cdot, \cdot, \cdot)$ are nondecreasing in the third variable, by their definitions at the beginning of Section 4.2. The first assertion follows from Theorem 5.9 applied to the family $\left(L_{n}\right)_{n}$ of the lifts of the components of $L$ to $\widetilde{M}$, if the dimension of $L$ is 1 , and from Theorem 5.7 otherwise.

To prove the last assertion, with the notation of Section 5.1, let $t_{e}=\sup _{x \in L} \mathrm{ht}_{e}(x)+\epsilon$. We add to the family of convex subsets in Theorem 5.7 if $\operatorname{dim} L \geq 2$, and in the proof of Theorem 5.9 otherwise, the family of horoballs $\gamma H_{e}\left[t_{e}\right]$ for $\gamma$ in $\Gamma$ (modulo the stabilizer $\Gamma_{\xi_{e}}$ ). Note that these horoballs have pairwise disjoint interiors, and that their interiors are disjoint from the $\epsilon$-neighbourhood of every lift of a component of $L$, so that Proposition 3.7 (4) does apply when the dimension of $L$ is 1.

Theorem 1.4 in the introduction follows from this one, by the above example (1).

Remarks (1) If we wanted to have the same locally geodesic line $\gamma$ for every cusp $e$ of $M$ in the second assertion of Corollary 5.10, we should add the bigger family of

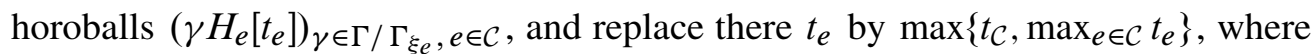
$\mathcal{C}$ is the set of cusps of $M$, and $t_{\mathcal{C}}$ is the greatest lower bound of $t \geq 0$ such that two distinct elements in $\left(\gamma H_{e}[t]\right)_{\gamma \in \Gamma / \Gamma_{\xi e}, e \in \mathcal{C}}$ have disjoint interiors (see the definition above Corollary 5.5), in order for the new horoballs to have disjoint interiors.

(2) With $M$ and $L$ as above, let $f$ be one of the symbols $\ell, \mathfrak{b} \mathfrak{p}, \mathfrak{f t p}, \mathfrak{c r p}$. Define, for every locally geodesic line $\gamma$ in $M$,

$$
\operatorname{maxspt}_{L, f}(\gamma)=\sup _{\alpha \in \mathcal{A}} f_{\alpha}(\tilde{\gamma}),
$$

the least upper bound of the spiraling times of $\gamma$ around $L$ with respect to $f$. Let

$$
\operatorname{MaxSp}_{L, f}(M)=\left\{\operatorname{maxspt}_{L, f}(\gamma): \gamma \in T^{1} M\right\}
$$


be the maximum spiraling spectrum $\operatorname{MaxSp}_{L, f}(M)$ around $L$ with respect to $f$. Corollary 5.10 gives, in particular, sufficient conditions for the maximum spiraling spectrum to contain a ray $[c,+\infty]$.

\subsection{Recurrent geodesics and related results}

In this subsection, when $M$ is geometrically finite, we construct locally geodesic lines that have a prescribed height in a cusp neighbourhood of $M$, and furthermore satisfy some recurrence properties. We will use the notation introduced in Section 5.1 concerning the cusps $e$, and the objects $\mathrm{ht}_{e}, V_{e}, H_{e}, \xi_{e}$.

Corollary 5.11 Let $M$ be a complete, nonelementary, geometrically finite Riemannian manifold with compact totally geodesic boundary, with sectional curvature at most -1 and dimension at least 3 . Let $e$ be a cusp of $M$. Then there exists a constant $c_{3}^{\prime \prime}=c_{3}^{\prime \prime}(e, M)$ such that for every $h^{\prime} \geq c_{3}^{\prime \prime}$, there exists a locally geodesic line $\gamma$ in $M$ with maxht $e_{e}(\gamma)=h^{\prime}$, such that the spiraling length times of $\gamma$ along the boundary $\partial M$ are at most $c_{3}^{\prime \prime}$.

Up to changing the constant $c_{3}^{\prime \prime}$, we may also assume that $\gamma$ stays away from some fixed (small enough) cusp neighbourhood of every cusp different from $e$. Note that, up to changing the constant $c_{3}^{\prime \prime}$, the last assertion of the corollary does not depend on the choice of $f=\ell, \mathfrak{b} \mathfrak{p}, \mathfrak{f t p}, \mathfrak{c r p}$, with respect to which the spiraling times are computed, and we will use $f=\ell$.

Proof As $\partial M$ is compact, there exists $\left.\epsilon^{\prime} \in\right] 0,1\left[\right.$ such that the $\epsilon^{\prime}$-neighbourhood of the geodesic boundary $\partial M$ is a tubular neighbourhood of $\partial M$. By definition of manifolds with totally geodesic boundary, there exists a complete simply connected Riemannian manifold $\widetilde{M}$, a nonelementary, torsion-free, geometrically finite discrete subgroup $\Gamma$ of isometries of $\widetilde{M}$, a $\Gamma$-equivariant collection $\left(L_{k}^{+}\right)_{k \in \mathbb{N}}$ of pairwise disjoint open halfspaces with totally geodesic boundary $\left(L_{k}\right)_{k \in \mathbb{N}}$, such that $M$ is isometric with $\Gamma \backslash\left(\widetilde{M}-\bigcup_{k \in \mathbb{N}} L_{k}^{+}\right)$. We will identify $M$ and $\Gamma \backslash\left(\widetilde{M}-\bigcup_{k \in \mathbb{N}} L_{k}^{+}\right)$ by such an isometry from now on. Note that $\left(\mathcal{N}_{\epsilon^{\prime}} L_{k}^{+}\right)_{k \in \mathbb{N}}$ is a family of pairwise disjoint $\epsilon^{\prime}$-convex subsets in $\widetilde{M}$.

Let $t_{e, \partial M}=\max \left\{0, \max _{x \in \partial M} \mathrm{ht}_{e}(x)\right\} \geq 0$, which exists since $\partial M$ is compact. Note that the family $\left(g H_{e}\left[t_{e, \partial M}+1\right]\right)_{g \in \Gamma / \Gamma_{\xi e}}$ is a $\Gamma$-equivariant family of pairwise disjoint horoballs in $\widetilde{M}$, which are disjoint from $\mathcal{N}_{\epsilon^{\prime}} L_{n}^{+}$for all $n \in \mathbb{N}$. Let us relabel this family of horoballs as $\left(H_{k}\right)_{k \in \mathbb{N}}$ such that $H_{0}=H_{e}\left[t_{e, \partial M}+1\right]$. Note that the horoballs $H_{k}, k \in \mathbb{N}$, are $\epsilon^{\prime}$-convex. 
Define

$$
c_{3}^{\prime \prime}=h_{1}^{\prime}\left(\epsilon^{\prime}, 0, h_{0}\left(\epsilon^{\prime}, 0, c_{1}^{\prime}(\infty)\right)\right)+t_{e, \partial M}+1+c_{1}^{\prime}\left(\epsilon^{\prime}\right)\left(c_{3}^{\prime}\left(\epsilon^{\prime}\right)+1\right),
$$

and let $h^{\prime} \geq c_{3}^{\prime \prime}$. We apply Corollary 4.11 with $X=\widetilde{M} ; \epsilon_{0}=\epsilon^{\prime} ; \delta_{0}=0 ; \kappa_{0}=c_{1}^{\prime}(\infty)$; $C_{0}=H_{0} ; f_{0}=\mathfrak{p h}_{C_{0}} ; h_{0}^{\prime}=h_{0}\left(\epsilon_{0}, \delta_{0}, \kappa_{0}\right) ; C_{2 k+1}=\mathcal{N}_{\epsilon^{\prime}} L_{k}^{+} ; C_{2 k}=H_{k} ; h=$ $2 h^{\prime}-2\left(t_{e, \partial M}+1\right)$. Note that $f_{0}$ is a $\kappa_{0}$-penetration map in $C_{0}$ by Lemma 3.3, and that $h \geq h_{1}^{\prime}\left(\epsilon_{0}, \delta_{0}, h_{0}^{\prime}\right)$, as $h^{\prime} \geq c_{3}^{\prime \prime}$. As $\widetilde{M}$ is a manifold of dimension at least 2, there does exist a geodesic line $\gamma_{0}$ in $X$ with $f_{0}\left(\gamma_{0}\right)=h$. The family $\left(C_{n}\right)_{n \in \mathbb{N}}$, whose elements have pairwise disjoint interiors, satisfies the assertion (iii). It also satisfies (iv), by Proposition 3.7 (1), as $h \geq 2 c_{3}^{\prime \prime}-2\left(t_{e, \partial M}+1\right) \geq 2 c_{1}^{\prime}\left(\epsilon^{\prime}\right)$. Hence, by Corollary 4.11, there exists a geodesic line $\widetilde{\gamma}$ in $X$ with $\mathfrak{p h}_{H_{0}}(\widetilde{\gamma})=h$ and

$$
\ell_{C_{n}}(\widetilde{\gamma}) \leq h_{1}^{\prime \prime}=h_{1}^{\prime}\left(\epsilon_{0}, \delta_{0}, h_{0}^{\prime}\right)+c_{1}^{\prime}\left(\epsilon_{0}\right)\left(c_{3}^{\prime}\left(\epsilon_{0}\right)+1\right) \leq c_{3}^{\prime \prime}
$$

for all $n \neq 0$.

As $\ell_{C_{2 n+1}}(\widetilde{\gamma})$ is finite, the geodesic $\tilde{\gamma}$ does not cross the boundary of $L_{n}^{+}$, so it stays in $\widetilde{M}-\bigcup_{k \in \mathbb{N}} L_{k}^{+}$. Let $\pi: \widetilde{M}-\bigcup_{k \in \mathbb{N}} L_{k}^{+} \rightarrow M$ be the canonical projection, and let $\gamma=\pi \circ \widetilde{\gamma}$. Hence, the length spiraling times of $\gamma$ along $\partial M$ are at most $c_{3}^{\prime \prime}$.

Note that

$$
\mathfrak{p h}_{H_{e}}(\widetilde{\gamma})=\mathfrak{p h}_{C_{0}}(\widetilde{\gamma})+2\left(t_{e, \partial M}+1\right)=h+2\left(t_{e, \partial M}+1\right)=2 h^{\prime},
$$

by the paragraph above Lemma 3.3. Furthermore, if $g \in\left(\Gamma-\Gamma_{\xi_{e}}\right) / \Gamma_{\xi_{e}}$, then there exists $k$ in $\mathbb{N}-\{0\}$ such that

$$
\begin{aligned}
\mathfrak{p h}_{g H_{e}}(\widetilde{\gamma}) & =\mathfrak{p h}_{C_{2 k}}(\widetilde{\gamma})+2\left(t_{e, \partial M}+1\right) \leq \ell_{C_{2 k}}(\widetilde{\gamma})+c_{1}^{\prime}(\infty)+2\left(t_{e, \partial M}+1\right) \\
& \leq h_{1}^{\prime \prime}+c_{1}^{\prime}(\infty)+2\left(t_{e, \partial M}+1\right) \leq 2 c_{3}^{\prime \prime} \leq 2 h^{\prime} .
\end{aligned}
$$

Thus maxht $\operatorname{mof}_{e}(\gamma)=h^{\prime}$ by the same proof as in the end of the proof of Collary 5.4. $\square$

Let $M$ be a compact, connected, orientable, irreducible, acylindrical, atoroidal, boundary incompressible 3-manifold with nonempty boundary (see for instance Matsuzaki and Taniguchi [44] for references on 3-manifolds and Kleinian groups). A hyperbolic structure on a manifold is a complete Riemannian metric with constant sectional curvature -1 . A cusp $e$ of a geometrically finite hyperbolic structure is maximal if the maximal Margulis neighbourhood of $e$ is a neighbourhood of an end of the manifold. Let $P$ be the union of the torus components of $\partial M$, and $\mathscr{G} \mathscr{F}(M)=\mathscr{G} \mathscr{F}(M, P)$ be the (nonempty) space of complete geometrically finite hyperbolic structures in the interior of $M$ whose cusps are maximal, up to isometries isotopic to the identity. Recall that $\mathscr{G} \mathscr{F}(M)$ is homeomorphic to the Teichmüller space of $\partial_{0} M=\partial M-P$. 
For every $\sigma$ in $\mathscr{\mathscr { F }}(M)$, the cusps of $\sigma$ are in one-to-one correspondence with the torus components of $\partial M$, as any minimizing geodesic ray representing a cusp converges to an end of the interior of $M$ corresponding to a torus component of $\partial M$. If $e$ is a torus component of $\partial M$, let $\operatorname{maxht}_{\sigma, e}(\gamma)$ denote the maximum height of a locally geodesic line $\gamma$ in $\sigma$ with respect to the cusp corresponding to $e$. The convex core of a structure $\sigma$ in $\mathscr{G} \mathscr{F}(M)$ is the smallest closed convex subset of the interior of $M$, whose injection in the interior of $M$ induces an isomorphism on the fundamental groups.

The following result generalizes Theorem 1.5 in the introduction to the case of several cusps.

Corollary 5.12 Let $M$ be a compact, connected, orientable, irreducible, acylindrical, atoroidal, boundary incompressible 3-manifold with boundary, and let e be a torus component of $\partial M$. For every compact subset $K$ in $\mathscr{G} \mathscr{F}(M)$, there exists a constant $c_{4}^{\prime \prime}=c_{4}^{\prime \prime}(K)$ such that for every $h \geq c_{4}^{\prime \prime}$ and every $\sigma \in K$, there exists a locally geodesic line $\gamma$ contained in the convex core of $\sigma$ such that $\operatorname{maxht}_{\sigma, e}(\gamma)=h$, and $\operatorname{maxht}_{\sigma, e^{\prime}}(\gamma) \leq c_{4}^{\prime \prime}$ for every torus component $e^{\prime} \neq e$ of $\partial M$.

Proof For a subset $A$ of $\partial_{\infty} \mathbb{H}_{\mathbb{R}}^{3}$, we denote by $\operatorname{Conv} A$ the hyperbolic convex hull of $A$ in $\mathbb{H}_{\mathbb{R}}^{3}$. A subgroup $\Gamma$ of $\pi_{1} M$ is called a boundary subgroup if there are an element $\gamma \in \pi_{1} M$, a component $C$ of $\partial_{0} M$, and a point $x \in C$ such that $\Gamma=\gamma \operatorname{Im}\left(\pi_{1}(C, x) \rightarrow\right.$ $\left.\pi_{1}(M, x)\right) \gamma^{-1}$. Let $\left(\Gamma_{n}\right)_{n \in \mathbb{N}}$ be the collection of boundary subgroups of $\pi_{1} M$. Let $\left(\Gamma_{n}^{\prime}\right)_{n \in \mathbb{N}}$ be the collection of maximal rank 2 abelian subgroups of $\pi_{1} M$, with $\Gamma_{0}^{\prime}$ conjugate to $\pi_{1} e$.

Let $\rho_{\sigma}: \pi_{1} M \rightarrow \operatorname{Isom}\left(\mathbb{H}_{\mathbb{R}}^{3}\right)$ be a holonomy representation corresponding to $\sigma \in K$, appropriately normalized to depend continuously on $\sigma$. By assumption, $\Gamma=\rho_{\sigma}\left(\pi_{1} M\right)$ is a (particular) web group (see for instance Abikoff and Maskit [1]). More precisely, for all $n \in \mathbb{N}, \rho_{\sigma}\left(\Gamma_{n}\right)$ is a quasifuchsian subgroup of $\Gamma$ stabilizing a connected, simply connected component $\Omega_{n, \sigma}$ of the domain of discontinuity of $\rho_{\sigma} \pi_{1} M$, such that $\Omega_{n, \sigma}$ and $\Omega_{m, \sigma}$ have disjoint closures if $n \neq m$, and that $\partial \Omega_{n, \sigma}$ contains no parabolic fixed points of $\Gamma$. Let $\left(H_{k, \sigma}\right)_{k \in \mathbb{N}}$ be a maximal family of horoballs with pairwise disjoint interiors such that $H_{k, \sigma}$ is $\rho_{\sigma}\left(\Gamma_{k}^{\prime}\right)$-invariant (such a family is unique if $M$ has only one torus component). To make this family canonical over $\mathscr{G} \mathscr{F}(M)$, we may fix an ordering $e_{1}=e, e_{2}, \ldots, e_{m}$ of the torus components of $\partial M$, and take by induction $H_{k, \sigma}$, for the $k$ 's in $\mathbb{N}$ such that $\Gamma_{k}^{\prime}$ is conjugate to $\pi_{1}\left(e_{i}\right)$, to be equivariant and maximal with respect to having pairwise disjoint interiors as well as having their interior disjoint with the interior of $H_{k_{*}, \sigma}$, for the $k_{*}$ 's in $\mathbb{N}$ such that $\Gamma_{k_{*}}^{\prime}$ is conjugate to $\pi_{1}\left(e_{j}\right)$ with $j<i$. Note that the $H_{k, \sigma}$ 's, besides the ones such that $\Gamma_{k}^{\prime}$ is conjugate to $\pi_{1} e$, may not be the horoballs that were used to define the height functions, but this changes their values only by a constant (uniform on $K$ ). 
Hence, as $K$ is compact, there exists $\delta>0$ such that for every $\sigma \in K$, the 1 -convex subsets $\mathcal{N}_{1}\left(\operatorname{Conv} \Omega_{n, \sigma}\right)$ and $H_{k, \sigma}$ for $n, k \in \mathbb{N}$ meet pairwise with diameter at most $\delta$.

The claim follows as in Corollary 5.11 by applying Corollary 4.11 to $X=\mathbb{H}_{\mathbb{R}}^{3}$, $\epsilon_{0}=1, \delta_{0}=\delta, \kappa_{0}=c_{1}^{\prime}(\infty), C_{0}=H_{0, \sigma}, f_{0}=\mathfrak{p h}_{C_{0}}, h_{0}^{\prime}=h_{0}\left(\epsilon_{0}, \delta_{0}, \kappa_{0}\right), C_{2 n+1}=$ $\mathcal{N}_{1}\left(\right.$ Conv $\left.\Omega_{n, \sigma}\right), C_{2 n}=H_{n, \sigma}$ to get a geodesic line $\widetilde{\gamma}$ in $X$ with prescribed penetration in $C_{0}$, and penetration bounded by a constant in $C_{n}$ for $n \neq 0$. The finiteness of the intersection lengths $\ell_{C_{2 n+1}}(\tilde{\gamma})$ for $n \in \mathbb{N}$ implies that $\tilde{\gamma}$ stays in the convex hull of the limit set of $\Gamma$.

Remark The fact that a locally geodesic line stays in the convex core of the manifold and does not converge (either way) to a cusp is equivalent with the locally geodesic line being two-sided recurrent.

\subsection{Prescribing the asymptotic penetration behaviour}

Let $X$ be a proper geodesic CAT(-1) metric space and let $\xi \in X \cup \partial_{\infty} X$. Let $\epsilon \in \mathbb{R}_{+}^{*} \cup\{\infty\}, \delta, \kappa \geq 0$. Let $\left(C_{\alpha}\right)_{\alpha \in \mathcal{A}}$ be a family of $\epsilon$-convex subsets of $X$ which satisfies the almost disjointness condition (iii) with parameter $\delta$. For each $\alpha \in \mathcal{A}$, let $f_{\alpha}$ be a $\kappa$-penetration map in $C_{\alpha}$. Let $\gamma$ be a geodesic ray or line, with 0 in the domain of definition of $\gamma$ (as we are only interested in the asymptotic behaviour, the choice of time 0 is unimportant). These assumptions guarantee that the set $\mathcal{E}_{\gamma}$ of times $t \geq 0$

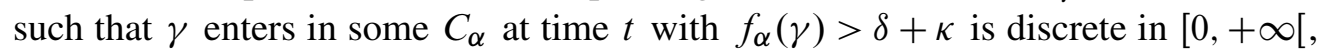
and that $\alpha=\alpha_{t}$ is then unique. The set $\mathcal{E}_{\gamma}$ may be finite, for instance if $f_{\beta}(\gamma)=+\infty$ for some $\beta$. Hence $\mathcal{E}_{\gamma}=\left(t_{i}\right)_{i \in \mathcal{N}}$ for some initial segment $\mathcal{N}$ in $\mathbb{N}$, with $t_{i}<t_{i+1}$ for $i, i+1$ in $\mathcal{N}$. With $a_{i}(\gamma)=f_{\alpha_{t_{i}}}(\gamma)$, the (finite or infinite) sequence $\left(a_{i}(\gamma)\right)_{n \in \mathcal{N}}$ will be called the (nonnegative) penetration sequence of $\gamma$ with respect to $\left(C_{\alpha}, f_{\alpha}\right)_{\alpha \in \mathcal{A}}$. In this section, we study the asymptotic behaviour of these penetration sequences. We will only state some results when the $C_{\alpha}$ 's are balls or horoballs, but similar ones are valid, for instance for $\epsilon$-neighbourhoods of geodesic lines in $X$ (see for instance our paper [49, Section 5]). We may also prescribe the asymptotic penetration in one cusp, while keeping the heights in the other cusps (uniformly) bounded.

In the following results, we show how to prescribe the asymptotic behaviour of the penetration sequence of a geodesic ray or line with respect to horoballs and their penetration height functions. First, we prove a general result, and we give the more explicit result for Riemannian manifolds as Corollary 5.14.

Theorem 5.13 Let $X$ be a proper geodesic CAT $(-1)$ metric space, with $\partial_{\infty} X$ infinite. Let $\left(H_{\alpha}\right)_{\alpha \in \mathcal{A}}$ be a family of horoballs with pairwise disjoint interiors. Assume that 
there exists $K \in\left[0,+\infty\left[\right.\right.$ and a dense subset $Y$ in $\partial_{\infty} X$ such that, for every geodesic ray $\gamma$ in $X$ with $\gamma(+\infty) \in Y$, we have

$$
\liminf _{t \rightarrow+\infty} d\left(\gamma(t), \bigcup_{\alpha \in \mathcal{A}} H_{\alpha}\right) \leq K .
$$

Let $\xi \in X \cup \partial_{\infty} X$ and $c, c^{\prime} \geq 0$. Assume that for every $h \geq c$ and $\alpha \in \mathcal{A}$ such that $\xi \notin H_{\alpha} \cup H_{\alpha}[\infty]$, there exists a geodesic ray or line $\gamma$ starting from $\xi$ and entering $H_{\alpha}$ at time $t=0$ with $\mathfrak{p h}_{H_{\alpha}}(\gamma)=h$, and with $\mathfrak{p h}_{H_{\beta}}(\gamma) \leq c^{\prime}$ for every $\beta$ in $\mathcal{A}-\{\alpha\}$ such that $\gamma(] 0,+\infty[)$ meets $H_{\beta}$. Let $\left(a_{i}\left(\gamma^{\prime}\right)\right)_{n \in \mathcal{N}}$ be the penetration sequence of a geodesic ray or line $\gamma^{\prime}$ with respect to $\left(H_{\alpha}, \mathfrak{p h}_{H_{\alpha}}\right)_{\alpha \in \mathcal{A}}$.

Then, for every

$$
h \geq h_{*}=\max \left\{c, c^{\prime}+3 c_{1}^{\prime}(\infty)+10^{-5}\right\},
$$

there exists a geodesic ray or line $\gamma$ starting from $\xi$ such that

$$
\limsup _{i \rightarrow+\infty} a_{i}(\gamma)=h \text {. }
$$

Proof To simplify notation, let $f_{\alpha}=\mathfrak{p h}_{H_{\alpha}}, c_{*}=c^{\prime}+3 c_{1}^{\prime}(\infty)+10^{-5}$, so that $h_{*}=\max \left\{c_{*}, c\right\}$. If a geodesic ray or line $\gamma$ starting from $\xi$ meets $H_{\alpha}$ such that $\xi \notin H_{\alpha} \cup H_{\alpha}[\infty]$, let $t_{\alpha}^{-}(\gamma)$ and $t_{\alpha}^{+}(\gamma)$ be the entrance and exit times.

Let $h \geq h_{*}$, and let $\alpha_{0} \in \mathcal{A}$ such that $\xi \notin H_{\alpha_{0}} \cup H_{\alpha_{0}}[\infty]$, which exists by the assumptions. As $h \geq h_{*} \geq c$, there exists a geodesic ray or line $\gamma_{0}$ starting from $\xi$, entering $H_{\alpha_{0}}$ at time 0 , such that $f_{\alpha_{0}}\left(\gamma_{0}\right)=h$, and $f_{\alpha}\left(\gamma_{0}\right) \leq c^{\prime}$ for every $\alpha \neq \alpha_{0}$ such that $\gamma_{0}(] 0,+\infty[)$ meets $H_{\alpha}$.

We construct, by induction, sequences $\left(\gamma_{k}\right)_{k \in \mathbb{N}}$ of geodesic rays or lines starting from $\xi,\left(\alpha_{k}\right)_{k \in \mathbb{N}}$ of elements of $\mathcal{A}$, and $\left(t_{k}\right)_{k \in \mathbb{N}-\{0\}}$ of elements in $[0,+\infty$ [ converging to $+\infty$, such that for every $k \in \mathbb{N}$,

(1) $\gamma_{k}$ enters the interior of $H_{\alpha_{0}}$ at time 0 , with $d\left(\gamma_{k}(0), \gamma_{k-1}(0)\right) \leq 1 / 2^{k}$ if $k \geq 1$,

(2) $\gamma_{k}$ enters $H_{\alpha_{k}}, \xi \notin H_{\alpha_{k}} \cup H_{\alpha_{k}}[\infty]$, and $f_{\alpha_{k}}\left(\gamma_{k}\right)=h$,

(3) if $0 \leq j \leq k-1$, then $\gamma_{k}(] 0,+\infty[)$ enters the interior of $H_{\alpha_{j}}$ before entering $H_{\alpha_{k}}$ with $t_{\alpha_{j}}^{-}\left(\gamma_{k}\right)<t_{k}=t_{\alpha_{k-1}}^{+}\left(\gamma_{k}\right)<t_{\alpha_{k}}^{+}\left(\gamma_{k}\right)$,

(4) if $k \geq 1$, then for every $\alpha$ such that $\gamma_{k}(] 0,+\infty[)$ meets $H_{\alpha}$, we have $\mid f_{\alpha}\left(\gamma_{k}\right)-$ $f_{\alpha}\left(\gamma_{k-1}\right) \mid<1 / 2^{k}$ if $t_{\alpha}^{-}\left(\gamma_{k}\right)<t_{k}$, and $f_{\alpha}\left(\gamma_{k}\right) \leq c_{*}$ if $t_{k} \leq t_{\alpha}^{-}\left(\gamma_{k}\right)<t_{\alpha_{k}}^{-}\left(\gamma_{k}\right)$, and $f_{\alpha}\left(\gamma_{k}\right) \leq c^{\prime}$ if $t_{\alpha}^{-}\left(\gamma_{k}\right) \geq t_{\alpha_{k}}^{+}\left(\gamma_{k}\right)$. 
Let us first prove that the existence of such sequences implies Theorem 5.13. By the assertion (1), as $\gamma_{k}(0)$ stays at bounded distance from $\gamma_{0}(0)$, up to extracting a subsequence, the sequence $\left(\gamma_{k}\right)_{k \in \mathbb{N}}$ converges to a geodesic ray or line $\gamma_{\infty}$ starting from $\xi$, entering in $H_{\alpha_{0}}$ at time $t=0$, by continuity of the entering point in an $\epsilon-$ convex subset. Let us prove that $\lim _{\sup } \rightarrow+\infty a_{i}\left(\gamma_{\infty}\right)=h$.

The lower bound $\lim \sup _{i \rightarrow+\infty} a_{i}\left(\gamma_{\infty}\right) \geq h$ is immediate by a semicontinuity argument. Indeed, for every $k>i$ in $\mathbb{N}$, we have by the assertions (2), (3) and (4),

$$
\left|f_{\alpha_{i}}\left(\gamma_{k}\right)-h\right|=\left|f_{\alpha_{i}}\left(\gamma_{k}\right)-f_{\alpha_{i}}\left(\gamma_{i}\right)\right| \leq \sum_{j=i}^{k-1}\left|f_{\alpha_{i}}\left(\gamma_{j+1}\right)-f_{\alpha_{i}}\left(\gamma_{j}\right)\right| \leq \sum_{j=i}^{k-1} \frac{1}{2^{j+1}} \leq \frac{1}{2^{i}} \text {. }
$$

Hence by continuity of $f_{\alpha_{i}}$, we have the inequality $f_{\alpha_{i}}\left(\gamma_{\infty}\right) \geq h-1 / 2^{i}$, whose right side converges to $h$ as $i$ tends to $+\infty$, which proves the lower bound, as $h>c_{1}^{\prime}(\infty)$ and $f_{\alpha_{i}}$ is a $c_{1}^{\prime}(\infty)$-penetration map in $H_{\alpha_{i}}$ (see Section 3.1).

To prove the upper bound, assume by contradiction that there exists $\epsilon>0$ such that for every $\lambda>0$, there exists $\alpha=\alpha(\lambda) \in \mathcal{A}$ such that $\gamma_{\infty}$ enters $H_{\alpha}$ with $f_{\alpha}\left(\gamma_{\infty}\right) \geq h+\epsilon$ and $t_{\alpha}^{-}\left(\gamma_{\infty}\right)>\lambda+2 c_{1}^{\prime}(\infty)$. Take $\lambda_{0}=\max \left\{t_{i+1}: 1 / 2^{i} \geq \frac{\epsilon}{2}\right\}$, and $\alpha=\alpha\left(\lambda_{0}\right)$

By continuity of $f_{\alpha}$, if $k$ is big enough, we have $f_{\alpha}\left(\gamma_{k}\right) \geq h+\frac{\epsilon}{2} \geq h_{*} \geq c_{*} \geq c^{\prime}$. Thus, $\gamma_{k}$ meets $H_{\alpha}$ as $h_{*}>0$. The entry time is positive, as $d\left(\gamma_{k}(0), \gamma_{\infty}(0)\right) \leq c_{1}^{\prime}(\infty)$ and the entrance points of $\gamma_{k}$ and $\gamma_{\infty}$ in $H_{\alpha}$ are at distance at most $c_{1}^{\prime}(\infty)$, both by Lemma 2.3, and as the entrance time of $\gamma_{\infty}$ in $H_{\alpha}$ is strictly bigger than $2 c_{1}^{\prime}(\infty)$. Hence, by the assertion (4), we have $t_{\alpha}^{-}\left(\gamma_{k}\right)<t_{k}$. Let $i \leq k-1$ be the minimum element of $\mathbb{N}$ such that for $j=i, \ldots, k-1$, the geodesic $\gamma_{j+1}$ meets $H_{\alpha}$ at a positive time with $t_{\alpha}^{-}\left(\gamma_{j+1}\right)<t_{j+1}$. By the triangle inequality, we have

$$
\begin{aligned}
\left|t_{\alpha}^{-}\left(\gamma_{i+1}\right)-t_{\alpha}^{-}\left(\gamma_{\infty}\right)\right| & \leq d\left(\gamma_{i+1}\left(t_{\alpha}^{-}\left(\gamma_{i+1}\right)\right), \gamma_{\infty}\left(t_{\alpha}^{-}\left(\gamma_{\infty}\right)\right)\right)+d\left(\gamma_{i+1}(0), \gamma_{\infty}(0)\right) \\
& \leq 2 c_{1}^{\prime}(\infty) .
\end{aligned}
$$

Hence $t_{i+1}>t_{\alpha}^{-}\left(\gamma_{i+1}\right) \geq t_{\alpha}^{-}\left(\gamma_{\infty}\right)-2 c_{1}^{\prime}(\infty)>\lambda_{0}+2 c_{1}^{\prime}(\infty)-2 c_{1}^{\prime}(\infty)=\lambda_{0}$.

By the definition of $\lambda_{0}$, we hence have $1 / 2^{i}<\frac{\epsilon}{2}$. By the definition of $i$ and by the assertion (4), we have

$$
\begin{aligned}
f_{\alpha}\left(\gamma_{i}\right) & =f_{\alpha}\left(\gamma_{k}\right)+\sum_{j=i}^{k-1}\left(f_{\alpha}\left(\gamma_{j}\right)-f_{\alpha}\left(\gamma_{j+1}\right)\right) \geq h+\frac{\epsilon}{2}-\sum_{j=i}^{k-1} \frac{1}{2^{j+1}} \\
& \geq h+\frac{\epsilon}{2}-\frac{1}{2^{i}} \geq h \geq h_{*},
\end{aligned}
$$


and in particular by the same argument as for $\gamma_{k}$ above, $\gamma_{i}$ enters $H_{\alpha}$ at a positive time and $t_{\alpha}^{-}\left(\gamma_{i}\right)<t_{i}$. This contradicts the minimality of $i$. This completes the proof, assuming the existence of the sequences with properties (1)-(4).

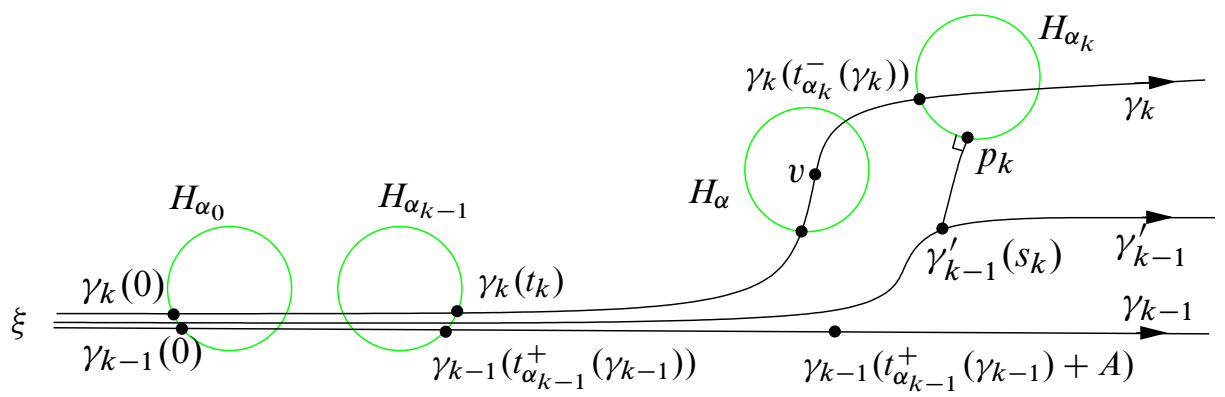

Let us now construct the sequences $\left(\gamma_{k}\right)_{k \in \mathbb{N}},\left(\alpha_{k}\right)_{k \in \mathbb{N}},\left(t_{k}\right)_{k \in \mathbb{N}-\{0\}}$. We have defined $\gamma_{0}, \alpha_{0}$, and they satisfy the properties (1)-(4). Let $k \geq 1$, and assume that $\gamma_{k-1}$, $\alpha_{k-1}$, as well as $t_{k-1}$ if $k \geq 2$, have been constructed.

As $Y$ is dense in $\partial_{\infty} X$, for every $A>0$, there exists a geodesic ray or line $\gamma_{k-1}^{\prime}$ starting from $\xi$ with $\gamma_{k-1}^{\prime}(+\infty) \in Y$, entering in $H_{\alpha_{0}}$ at time $t=0$, which is very close to $\gamma_{k-1}$ on $\left[0, t_{\alpha_{k-1}}^{+}\left(\gamma_{k-1}\right)+A\right]$. By the definition of $K$, let $s_{k}$ be the first time $t \geq t_{\alpha_{k-1}}^{+}\left(\gamma_{k-1}\right)+A$ such that there exists $\alpha$ in $\mathcal{A}$ with $d\left(\gamma_{k-1}^{\prime}(t), H_{\alpha}\right) \leq K+1$, and let $\alpha_{k}$ be such an $\alpha$ with $d\left(\gamma_{k-1}^{\prime}\left(s_{k}\right), H_{\alpha}\right)$ minimum. Let $p_{k}$ be the closest point of $H_{\alpha_{k}}$ to $\gamma_{k-1}^{\prime}\left(s_{k}\right)$. Note that $\xi \notin H_{\alpha_{k}} \cup H_{\alpha_{k}}[\infty]$, if $A$ is big enough (in particular compared to $K$ ), as $H_{\alpha_{0}}$ and $H_{\alpha_{k}}$ have disjoint interiors.

By the hypothesis, let $\gamma_{k}$ be a geodesic ray or line starting from $\xi$ with $f_{\alpha_{k}}\left(\gamma_{k}\right)=h$ (which proves the assertion (2) at rank $k$ as $h>0$ ) and $f_{\alpha}\left(\gamma_{k}\right) \leq c^{\prime}$ for every $\alpha$ such that $\gamma_{k}(] t_{\alpha_{k}}^{-}\left(\gamma_{k}\right),+\infty[)$ enters $H_{\alpha}$. As a CAT $(-1)$ metric space is $\log (1+\sqrt{2})$-hyperbolic, the geodesic $\left.] \xi, p_{k}\right]$ is contained in the $\log (1+\sqrt{2})$-neighbourhood of the union ]$\left.\xi, \gamma_{k-1}^{\prime}\left(s_{k}\right)\right] \cup\left[\gamma_{k-1}^{\prime}\left(s_{k}\right), p_{k}\right]$. By Lemma 2.3, we have $\left.\left.d\left(\gamma_{k}\left(t_{\alpha_{k}}^{-}\left(\gamma_{k}\right)\right),\right] \xi, p_{k}\right]\right) \leq$ $c_{1}^{\prime}(\infty)$, and therefore $\left.] \xi, \gamma_{k}\left(t_{\alpha_{k}}^{-}\left(\gamma_{k}\right)\right)\right]$ is contained in the $\left(c_{1}^{\prime}(\infty)+\log (1+\sqrt{2})\right)-$ neighbourhood of $\left.] \xi, \gamma_{k-1}^{\prime}\left(s_{k}\right)\right] \cup\left[\gamma_{k-1}^{\prime}\left(s_{k}\right), p_{k}\right]$. Up to choosing $A$ big enough, we may hence assume $\gamma_{k}$ is very close to $\gamma_{k-1}$ between the times 0 and $t_{\alpha_{k-1}}^{+}\left(\gamma_{k-1}\right)+1$. Using this and properties (1) and (3) at rank $k-1$, we have

- $\gamma_{k}$ does enter the interior of $H_{\alpha_{0}}$, at a time that we may assume to be 0 , with $d\left(\gamma_{k}(0), \gamma_{k-1}(0)\right) \leq 1 / 2^{k}$ (this proves the assertion (1) at rank $k$ );

- for $0 \leq j \leq k-1$, as $\gamma_{k-1}$ passes in the interior of $H_{\alpha_{j}}$ at a time strictly between 0 and $t_{\alpha_{k-1}}^{+}\left(\gamma_{k-1}\right)$, by the inductive assertions (3) if $k \neq 1$ and $j \leq k-2$, or (1) if $k=1$ or (2) if $j=k-1$, so does the geodesic ray or line $\gamma_{k}$; this allows, 
in particular, to define $t_{k}=t_{\alpha_{k-1}}^{+}\left(\gamma_{k}\right)$, which satisfies $t_{k} \leq t_{\alpha_{k}}^{-}\left(\gamma_{k}\right)<t_{\alpha_{k}}^{+}\left(\gamma_{k}\right)$ and

$$
d\left(\gamma_{k}\left(t_{k}\right), \gamma_{k-1}\left(t_{\alpha_{k-1}}^{+}\left(\gamma_{k-1}\right)\right)\right) \leq 10^{-5} / 4
$$

if $A$ is big enough; this proves the assertion (3) at rank $k$;

- for every $\alpha$ such that $\gamma_{k}(] 0,+\infty[)$ meets $H_{\alpha}$ and $t_{\alpha}^{-}\left(\gamma_{k}\right)<t_{k}$, we may assume by continuity that $\left|f_{\alpha}\left(\gamma_{k}\right)-f_{\alpha}\left(\gamma_{k-1}\right)\right|<1 / 2^{k}$.

Hence, to prove the assertion (4) at rank $k$, we consider $\alpha \in \mathcal{A}$ such that $\gamma_{k}$ meets $H_{\alpha}$ with $t_{k} \leq t_{\alpha}^{-}\left(\gamma_{k}\right)<t_{\alpha_{k}}^{-}\left(\gamma_{k}\right)$, and we prove that $f_{\alpha}\left(\gamma_{k}\right) \leq c_{*}$. We may assume that $f_{\alpha}\left(\gamma_{k}\right)>0$. Let $v$ be the highest point of $\gamma_{k}$ in $H_{\alpha}$, which, by disjointness, belongs to $] \gamma_{k}\left(t_{\alpha}^{-}\left(\gamma_{k}\right)\right), \gamma_{k}\left(t_{\alpha_{k}}^{-}\left(\gamma_{k}\right)\right)[$. Let $u$ be a point in $\left.] \xi, \gamma_{k-1}^{\prime}\left(s_{k}\right)\right] \cup\left[\gamma_{k-1}^{\prime}\left(s_{k}\right), p_{k}\right]$ at distance at most $c_{1}^{\prime}(\infty)+\log (1+\sqrt{2})=\frac{3}{2} c_{1}^{\prime}(\infty)$ from $v$.

Assume first that $u \in\left[\gamma_{k-1}^{\prime}\left(s_{k}\right), p_{k}\right]$. Note that by the minimality assumption on $\alpha_{k}$ and since $\alpha \neq \alpha_{k}$, the point $u$ then does not belong to $H_{\alpha}$. As $c_{*} \geq 3 c_{1}^{\prime}(\infty)$, this implies that $f_{\alpha}\left(\gamma_{k}\right) \leq 2 d(u, v) \leq c_{*}$.

Assume now that $u=\gamma_{k-1}^{\prime}(t)$ with $t \in\left[t_{\alpha_{k-1}}^{+}\left(\gamma_{k-1}\right)+A, s_{k}[\right.$. Then by the minimality of $s_{k}$, the point $u$ again does not belong to $H_{\alpha}$ (it is in fact at distance at least $K+1$ from $H_{\alpha}$ ). Hence similarly $f_{\alpha}\left(\gamma_{k}\right) \leq c_{*}$.

Finally, assume that $u=\gamma_{k-1}^{\prime}(t)$ with $t \leq t_{\alpha_{k-1}}^{+}\left(\gamma_{k-1}\right)+A$. Let $u^{\prime}=\gamma_{k-1}(t)$, which satisfies $d\left(u, u^{\prime}\right) \leq 10^{-5} / 4$ (as $\gamma_{k-1}$ and $\gamma_{k-1}^{\prime}$ were assumed to be very close on that range). Assume by contradiction that $f_{\alpha}\left(\gamma_{k}\right)>c_{*}$. In particular,

$$
d\left(v, \gamma_{k}\left(t_{k}\right)\right) \geq d\left(v, \gamma_{k}\left(t_{\alpha}^{-}\left(\gamma_{k}\right)\right)\right) \geq f_{\alpha}\left(\gamma_{k}\right) / 2>c_{*} / 2 .
$$

Let $\xi_{\alpha}$ be the point at infinity of $H_{\alpha}$ and $x_{\alpha}=\gamma_{k}\left(t_{\alpha}^{-}\left(\gamma_{k}\right)\right)$ the entrance point of $\gamma_{k}$ in $H_{\alpha}$. Since $d\left(v, u^{\prime}\right) \leq d(v, u)+d\left(u, u^{\prime}\right) \leq \frac{3}{2} c_{1}^{\prime}(\infty)+10^{-5} / 4$, we hence have

$$
\begin{aligned}
2 \beta_{\xi_{\alpha}}\left(u^{\prime}, x_{\alpha}\right) & =2 \beta_{\xi_{\alpha}}\left(u^{\prime}, v\right)+2 \beta_{\xi_{\alpha}}\left(v, x_{\alpha}\right) \\
& \geq f_{\alpha}\left(\gamma_{k}\right)-2 d\left(v, u^{\prime}\right) \geq c_{*}-3 c_{1}^{\prime}(\infty)-10^{-5} / 2>c^{\prime} \geq 0,
\end{aligned}
$$

by the definition of $c_{*}$. Hence $u^{\prime} \in H_{\alpha}$ and $f_{\alpha}\left(\gamma_{k-1}\right) \geq 2 \beta_{\xi_{\alpha}}\left(u^{\prime}, x_{\alpha}\right)>c^{\prime}$. Furthermore, $t \geq t_{\alpha_{k-1}}^{+}\left(\gamma_{k-1}\right)$, since otherwise, and by Equation (40),

$\left.d\left(\gamma_{k}\left(t_{k}\right)\right), v\right) \leq d\left(v, u^{\prime}\right)+d\left(\gamma_{k}\left(t_{k}\right), \gamma_{k-1}\left(t_{\alpha_{k-1}}^{+}\left(\gamma_{k-1}\right)\right)\right) \leq \frac{3}{2} c_{1}^{\prime}(\infty)+10^{-5} / 2 \leq c_{*} / 2$ by the definition of $c_{*}$, a contradiction. Hence, by convexity and since $H_{\alpha}$ and $H_{\alpha_{k-1}}$ have disjoint interiors, we have $t_{\alpha}^{-}\left(\gamma_{k-1}\right) \geq t_{\alpha_{k-1}}^{+}\left(\gamma_{k-1}\right)$. Since $f_{\alpha}\left(\gamma_{k-1}\right)>c^{\prime}$, this contradicts the inductive hypothesis (4) on $\gamma_{k-1}$. This proves the result. 
Remark There is an analogous statement, with an analogous proof, when $\left(H_{\alpha}\right)_{\alpha \in \mathcal{A}}$ is a family of balls of radius $R>0$, replacing $c_{1}^{\prime}(\infty)$ by $c_{1}^{\prime}(R)$, and assuming both in the hypothesis and in the conclusion that $h \leq c^{\prime \prime}$ for some $c^{\prime \prime}$.

Corollary 5.14 Let $X$ be a complete simply connected Riemannian manifold with sectional curvature at most -1 and dimension at least 3 , and let $\left(H_{\alpha}\right)_{\alpha \in \mathcal{A}}$ be a family of horoballs in $X$ with disjoint interiors. Assume that there exists $K \in[0,+\infty[$ and a dense subset $Y$ in $\partial_{\infty} X$ such that, for every geodesic ray $\gamma$ in $X$ with $\gamma(+\infty) \in Y$, we have $\liminf _{t \rightarrow+\infty} d\left(\gamma(t), \bigcup_{\alpha \in \mathcal{A}} H_{\alpha}\right) \leq K$. Then, for every $\xi \in X \cup \partial_{\infty} X$ and

$$
h \geq c_{1}^{\prime \prime}(\infty, 0,0)+4 c_{1}^{\prime}(\infty)+10^{-5} \approx 13.5542,
$$

there exists a geodesic ray or line $\gamma$ starting from $\xi$ such that, with $\left(a_{i}(\gamma)\right)_{n \in \mathcal{N}}$ the penetration sequence of $\gamma$ with respect to $\left(H_{\alpha}, \mathfrak{p h}_{H_{\alpha}}\right)_{\alpha \in \mathcal{A}}$, we have

$$
\limsup _{i \rightarrow+\infty} a_{i}(\gamma)=h \text {. }
$$

Proof Let $c=c_{1}^{\prime \prime}(\infty, 0,0), c^{\prime}=c_{1}^{\prime \prime}(\infty, 0,0)+c_{1}^{\prime}(\infty)$. We apply Theorem 5.1 with $\epsilon=\infty, \delta=0, \kappa=c_{1}^{\prime}(\infty), \xi_{0}=\xi, C_{0}=H_{\alpha}$ where $\alpha \in \mathcal{A}$ satisfies $\xi \notin H_{\alpha} \cup H_{\alpha}[\infty]$, $f_{0}=\mathfrak{p h}_{C_{0}},\left(C_{n}\right)_{n \geq 1}$ is $\left(H_{\beta}\right)_{\beta \in \mathcal{A}-\{\alpha\}}$ (up to indexing), $f_{n}=\mathfrak{p h}_{C_{n}}$ for every $n \in \mathbb{N}$ such that $\xi \notin C_{n} \cup \partial_{\infty} C_{n}$. Then the assumptions of Theorem 5.13 are satisfied. An easy computation of $h_{*}$ in Theorem 5.13 then yields the result.

Remark Using Theorem 5.2 instead of Theorem 5.1, there is an analogous statement when $\left(H_{\alpha}\right)_{\alpha \in \mathcal{A}}$ is a family of balls of radius $R>0$, for $h \in\left[c_{1}^{\prime \prime}(R, 0,0)+4 c_{1}^{\prime}(R)+10^{-5}\right.$, $\left.2 R-c_{1}^{\prime}(R)\right]$.

As in Section 5.1, we consider a complete, nonelementary, geometrically finite Riemannian manifold $M$, and $e$ an end of $M$ endowed with a choice of a Margulis neighborhood. The asymptotic height spectrum of the pair $(M, e)$ is

$$
\operatorname{LimsupSp}(M, e)=\left\{\limsup _{t \rightarrow \infty} \mathrm{ht}_{e}(\gamma(t)): \gamma \in T^{1} M\right\} .
$$

In classical Diophantine approximation, the Lagrange spectrum is defined as the subset of $[0,+\infty[$ consisting of the approximation constants $c(x)$ of an irrational real number $x$ by rational numbers $p / q$, defined by

$$
c(x)=\liminf _{q \rightarrow \infty}|q|^{2}\left|x-\frac{p}{q}\right| .
$$

Using the well known connection between the Diophantine approximation of real numbers by rational numbers and the action of the modular group $\mathrm{PSL}_{2}(\mathbb{Z})$ on the 
upper halfplane model of the real hyperbolic plane, the asymptotic height spectrum of the modular orbifold $\operatorname{PSL}_{2}(\mathbb{Z}) \backslash \mathbb{H}_{\mathbb{R}}^{2}$ is the image of the Lagrange spectrum by the map $t \mapsto-\log 2 t$ (see for instance Hersonsky and Paulin [30, Theorem 3.4]). Hall $[24 ; 25]$ showed that the Lagrange spectrum contains an interval $[0, c]$ for some $c>0$. The maximal such interval $[0, \mu]$ (which is closed as the Lagrange spectrum is closed, by Cusick's result (see for instance Cusick and Flahive [15]), called Hall's ray, was determined by Freiman [21] (see also Sloane [58] where the map $t \mapsto 1 / t$ has to be applied). The geometric interpretation of Freiman's result in our context is that LimsupSp $\left(\mathrm{PSL}_{2}(\mathbb{Z}) \backslash \mathbb{H}_{\mathbb{R}}^{2}\right)$ contains the maximal interval $[c,+\infty]$ with

$$
c=-\log (2 \mu)=-\log 2\left(\frac{491993569}{2221564096+283748 \sqrt{462}}\right) \approx 0.817 .
$$

The next result is the asymptotic analog of Corollary 5.4, and has a completely similar proof. Theorem 1.6 in the introduction follows, since $\left(c_{1}^{\prime \prime}(\infty, 0,0)+4 c_{1}^{\prime}(\infty)+10^{-5}\right) / 2$ $\approx 6.7771$. The result proves the existence of Hall's ray in our geometric context, which is much more general than the above particular case; there is no assumption of arithmetic nature, nor of constant curvature nature. Furthermore, we obtain a universal constant (though we do not know the optimal one) 6.7771 which is not too far from the geometric Freiman constant 0.817 .

Corollary 5.15 Let $M$ be a complete, nonelementary, geometrically finite Riemannian manifold with sectional curvature at most -1 and dimension at least 3 , and let $e$ be a cusp of $M$. Then $\operatorname{LimsupSp}(M, e)$ contains the interval

$$
\left[\left(c_{1}^{\prime \prime}(\infty, 0,0)+4 c_{1}^{\prime}(\infty)+10^{-5}\right) / 2,+\infty\right] .
$$

\section{Applications to Diophantine approximation in negatively curved manifolds}

In this section, we consider a number of arithmetically defined examples, illustrating the last result, Corollary 5.15. But we need first to recall some properties and do some computations in the real and complex hyperbolic spaces.

\subsection{On complex hyperbolic geometry and the Heisenberg group}

To facilitate computations, we identify elements in $\mathbb{C}^{n-1}$ with their coordinate column matrices. We will denote by $A^{*}={ }^{t} \bar{A}$ the adjoint matrix of a complex matrix $A$. In particular, the standard Hermitian scalar product of $w, w^{\prime} \in \mathbb{C}^{n-1}$ is $\overline{w^{*} w^{\prime}}=$ $\sum_{i=1}^{n-1} w_{i} \overline{w_{i}^{\prime}}$. We also use the notation $|w|^{2}=w^{*} w$. 
Let $\mathbb{H}_{\mathbb{C}}^{n}$ be the Siegel domain model of the complex hyperbolic $n$-space, whose underlying set is

$$
\mathbb{H}_{\mathbb{C}}^{n}=\left\{\left(w_{0}, w\right) \in \mathbb{C} \times \mathbb{C}^{n-1}: 2 \operatorname{Re} w_{0}-|w|^{2}>0\right\},
$$

and whose Riemannian metric is

$d s_{\mathbb{C}}^{2}=\frac{4}{\left(2 \operatorname{Re} w_{0}-|w|^{2}\right)^{2}}\left(\left(d w_{0}-d w^{*} w\right)\left(\overline{d w_{0}}-w^{*} d w\right)+\left(2 \operatorname{Re} w_{0}-|w|^{2}\right) d w^{*} d w\right)$,

(see for instance Goldman [23, Section 4.1]). The complex hyperbolic space has constant holomorphic sectional curvature -1 , hence its real sectional curvatures are bounded between -1 and $-\frac{1}{4}$. Its boundary at infinity is

$$
\partial_{\infty} \mathbb{H}_{\mathbb{C}}^{n}=\left\{\left(w_{0}, w\right) \in \mathbb{C} \times \mathbb{C}^{n-1}: 2 \operatorname{Re} w_{0}-|w|^{2}=0\right\} \cup\{\infty\} .
$$

The horoballs centered at $\infty$ in $\mathbb{H}_{\mathbb{C}}^{n}$ are the subsets

$$
\mathcal{H}_{s}=\left\{\left(w_{0}, w\right) \in \mathbb{C} \times \mathbb{C}^{n-1}: 2 \operatorname{Re} w_{0}-|w|^{2} \geq s\right\},
$$

for $s>0$. Note that the subset $\mathbb{H}_{\mathbb{C}}^{1}=\left\{\left(w_{0}, w\right) \in \mathbb{H}_{\mathbb{C}}^{n}: w=0\right\}$ is the right halfplane model of the real hyperbolic plane with constant curvature -1 , and it is totally geodesic in $\mathbb{H}_{\mathbb{C}}^{n}$. In particular, the (unit speed) geodesic line starting from $\infty$, ending at $(0,0) \in \partial_{\infty} \mathbb{H}_{\mathbb{C}}^{n}$ and meeting the horosphere $\partial \mathcal{H}_{2}$ at time $t=0$ is the map $c_{0}: \mathbb{R} \rightarrow \mathbb{H}_{\mathbb{C}}^{n}$ defined by $c_{0}: t \mapsto\left(e^{-t}, 0\right)$.

Let $q$ be the nondegenerate Hermitian form $-z_{0} \overline{z_{n}}-z_{n} \overline{z_{0}}+|z|^{2}$ of signature $(1, n)$ on $\mathbb{C} \times \mathbb{C}^{n-1} \times \mathbb{C}$ with coordinates $\left(z_{0}, z, z_{n}\right)$. This is not the form considered in [23, page 67], hence we need to do some computations with it, but it is better suited for our purposes. The Siegel domain $\mathbb{H}_{\mathbb{C}}^{n}$ embeds in the complex projective $n$-space $\mathbb{P}_{n}(\mathbb{C})$ by the map (using homogeneous coordinates)

$$
\left(w_{0}, w\right) \mapsto\left[w_{0}: w: 1\right] .
$$

Its image is the negative cone of $q$, that is $\left\{\left[z_{0}: z: z_{n}\right] \in \mathbb{P}_{n}(\mathbb{C}): q\left(z_{0}, z, z_{n}\right)<0\right\}$. This embedding extends continuously to the boundary at infinity, by mapping $\left(w_{0}, w\right) \in$ $\partial_{\infty} \mathbb{H}_{\mathbb{C}}^{n}-\{\infty\}$ to $\left[w_{0}: w: 1\right]$ and $\infty$ to $[1: 0: 0]$, so that the image of $\partial_{\infty} \mathbb{H}_{\mathbb{C}}^{n}$ is the null cone of $q$, that is $\left\{\left[z_{0}: z: z_{n}\right] \in \mathbb{P}_{n}(\mathbb{C}): q\left(z_{0}, z, z_{n}\right)=0\right\}$. We use matrices by blocks in the decomposition $\mathbb{C} \times \mathbb{C}^{n-1} \times \mathbb{C}$.

Let

$$
Q=\left(\begin{array}{ccc}
0 & 0 & -1 \\
0 & I & 0 \\
-1 & 0 & 0
\end{array}\right)
$$


be the matrix of $q$. If

then

$$
\begin{aligned}
X & =\left(\begin{array}{ccc}
a & \gamma^{*} & b \\
\alpha & A & \beta \\
c & \delta^{*} & d
\end{array}\right), \\
Q^{-1} X^{*} Q & =\left(\begin{array}{ccc}
\bar{d} & -\beta^{*} & \bar{b} \\
-\delta & A^{*} & -\gamma \\
\bar{c} & -\alpha^{*} & \bar{a}
\end{array}\right) .
\end{aligned}
$$

If $\mathrm{U}_{Q}$ is the group of $(n+1) \times(n+1)$ invertible matrices with complex coefficients preserving the Hermitian form $q$, then $X$ belongs to $\mathrm{U}_{Q}$ if and only if $X$ is invertible with inverse $Q^{-1} X^{*} Q$. In particular, if $X$ belongs to $\mathrm{U}_{Q}$, then

$$
\left\{\begin{aligned}
c \bar{d}-\delta^{*} \delta+d \bar{c} & =0, \\
a \bar{b}-\gamma^{*} \gamma+b \bar{a} & =0, \\
-\alpha \beta^{*}+A A^{*}-\beta \alpha^{*} & =I, \\
c \bar{b}-\delta^{*} \gamma+d \bar{a} & =1, \\
\bar{d} \alpha-A \delta+\bar{c} \beta & =0, \\
\bar{b} \alpha-A \gamma+\bar{a} \beta & =0 .
\end{aligned}\right.
$$

The group $\mathrm{U}_{Q}$ acts projectively on $\mathbb{P}_{n}(\mathbb{C})$, preserving the negative cone of $q$, hence it acts on $\mathbb{H}_{\mathbb{C}}^{n}$. We will denote in the same way the action of $U_{Q}$ on $\mathbb{H}_{\mathbb{C}}^{n}$ and the action of $U_{Q}$ on the image of $\mathbb{H}_{\mathbb{C}}^{n}$ in $\mathbb{P}_{n}(\mathbb{C})$. It is well known (see for instance Goldman [23]) that $\mathrm{U}_{Q}$ preserves the Riemannian metric of $\mathbb{H}_{\mathbb{C}}^{n}$.

The Heisenberg group Heis $_{2 n-1}$ is the real Lie group with the underlying space $\mathbb{C}^{n-1} \times \mathbb{R}$ and the group law

$$
(\zeta, v)\left(\zeta^{\prime}, v^{\prime}\right)=\left(\zeta+\zeta^{\prime}, v+v^{\prime}-2 \operatorname{Im} \zeta^{*} \zeta^{\prime}\right)
$$

It has a Lie group embedding in $\mathrm{U}_{Q}$, defined by

$$
(\zeta, v) \mapsto u_{\zeta, v}=\left(\begin{array}{ccc}
1 & \zeta^{*} & \frac{|\zeta|^{2}}{2}-i \frac{v}{2} \\
0 & I & \zeta \\
0 & 0 & 1
\end{array}\right),
$$

whose image preserves the point $\infty$ as well as each horoball centered at $\infty$, as an easy computation shows.

The Cygan distance (see Goldman [23, page 160]) on Heis $2 n-1$ is the unique leftinvariant distance $d_{\text {Cyg }}$ such that

$$
d_{\text {Cyg }}((0,0),(\zeta, v))=\left(|\zeta|^{4}+v^{2}\right)^{1 / 4} .
$$


We introduce the modified Cygan distance $d_{\text {Cyg }}^{\prime}$ as the unique left-invariant distance $d_{\text {Cyg }}^{\prime}$ such that

$$
d_{\text {Cyg }}^{\prime}((0,0),(\zeta, v))=\left(\left(|\zeta|^{4}+v^{2}\right)^{1 / 2}+|\zeta|^{2}\right)^{1 / 2} .
$$

It is straightforward to check that $d_{\text {Cyg }}^{\prime}$ is indeed a distance, in the same way as the Cygan distance (see for instance Korányi and Reimann [41, page 320]) and that it is equivalent to the Cygan distance,

$$
d_{\text {Cyg }} \leq d_{\text {Cyg }}^{\prime} \leq \sqrt{2} d_{\text {Cyg }} .
$$

Hence, its induced length distance is equivalent to the Carnot-Carathéodory distance on the Heisenberg group Heis $2 n-1$ (see Goldman [23, page 161]).

As the action of $\operatorname{Heis}_{2 n-1}$ on $\partial_{\infty} \mathbb{H}_{\mathbb{C}}^{n}-\{\infty\}$ is simply transitive, $d_{\text {Cyg }}$ and $d_{\text {Cyg }}^{\prime}$ define distances on $\partial_{\infty} \mathbb{H}_{\mathbb{C}}^{n}-\{\infty\}$, which are invariant under the action of Heis ${ }_{2 n-1}$. We also call these distances the Cygan distance and the modified Cygan distance, and again denote them by $d_{\text {Cyg }}$ and $d_{\text {Cyg }}^{\prime}$. Explicitly, these distances are given by

$$
d_{\mathrm{Cyg}}\left(u_{\zeta, v}(0,0), u_{\zeta^{\prime}, v^{\prime}}(0,0)\right)=d_{\mathrm{Cyg}}\left((\zeta, v),\left(\zeta^{\prime}, v^{\prime}\right)\right),
$$

and the similar expression for the modified Cygan distance.

Lemma 6.1 The distance $d_{\text {Cyg }}$ (resp. $d_{\text {Cyg }}^{\prime}$ ) is the unique distance on $\partial_{\infty} \mathbb{H}_{\mathbb{C}}^{n}-\{\infty\}$ invariant under the action of $\operatorname{Heis}_{2 n-1}$ such that $d_{\text {Cyg }}\left(\left(w_{0}, w\right),(0,0)\right)=\sqrt{2\left|w_{0}\right|}$ (resp. $\left.d_{\text {Cyg }}^{\prime}\left(\left(w_{0}, w\right),(0,0)\right)=\sqrt{2\left|w_{0}\right|+|w|^{2}}\right)$.

Proof For every $\left(w_{0}, w\right)$ in $\partial_{\infty} \mathbb{H}_{\mathbb{C}}^{n}-\{\infty\}$, note that $\left(w_{0}, w\right)=u_{\zeta, v}(0,0)$ if and only if $v=-2 \operatorname{Im} w_{0}$ and $\zeta=w$, and that $2 \operatorname{Re} w_{0}=|w|^{2}$. Hence

$$
d_{\text {Cyg }}^{\prime}\left(u_{\zeta, v}(0,0),(0,0)\right)=\left(\left(4 \operatorname{Re}^{2} w_{0}+4 \operatorname{Im}^{2} w_{0}\right)^{1 / 2}+|w|^{2}\right)^{1 / 2}=\sqrt{2\left|w_{0}\right|+|w|^{2}} .
$$

A similar proof gives the result for the Cygan distance.

In particular, if $n=2$, then $d_{\text {Cyg }}^{\prime}$ is indeed defined as in the statement of Theorem 1.8 in the introduction.

Let $d_{\mathbb{H}_{\mathbb{C}}^{n}}$ be the Riemannian distance on $\mathbb{H}_{\mathbb{C}}^{n}$, and $d_{\mathbb{H}_{\mathbb{C}}^{n}}^{\prime}=\frac{1}{2} d_{\mathbb{H}_{\mathbb{C}}^{n}}$ be the Riemannian distance of the Riemannian metric of $\mathbb{H}_{\mathbb{C}}^{n}$ renormalized to have maximal real sectional curvatures -1 .

Proposition 6.2 For every $\xi, \xi^{\prime}$ in $\partial_{\infty} \mathbb{H}_{\mathbb{C}}^{n}-\{\infty\}$, for every $s_{0}>0$, the distance $\ell^{\prime}$ for the renormalized Riemannian distance $d_{\mathbb{H}_{\mathbb{C}}^{n}}^{\prime}$ between the horoball $\mathcal{H}_{s_{0}}$ and the horoball 
centered at $\xi$ and tangent to the geodesic line between $\infty$ and $\xi^{\prime}$ is, if these horoballs are disjoint,

$$
\ell^{\prime}=-\log d_{\text {Cyg }}^{\prime}\left(\xi, \xi^{\prime}\right)+\frac{1}{2} \log \left(\frac{s_{0}}{2}\right)
$$

Proof By invariance of the modified Cygan distance, of each horoball centered at $\infty$, and of the normalized Riemannian distance, under the action of the Heisenberg group, we may assume that $\xi=(0,0)$. Let $(\zeta, v) \in \operatorname{Heis}_{2 n-1}$ such that $\xi^{\prime}=u_{\zeta, v}(\xi)$. As $u_{\zeta, v}$ sends geodesic lines to geodesic lines, and fixes $\infty$, the geodesic lines (for $d_{\mathbb{H}_{\mathbb{C}}^{n}}$ ) starting from $\infty$ and ending at $\xi^{\prime}$ are time translates of $u_{\zeta, v} \circ c_{0}$, which by an easy computation is

$$
u_{\zeta, v} \circ c_{0}: t \mapsto\left(e^{-t}+\left(|\zeta|^{2}-i v\right) / 2, \zeta\right)
$$

The matrix

$$
X_{0}=\left(\begin{array}{lll}
0 & 0 & 1 \\
0 & I & 0 \\
1 & 0 & 0
\end{array}\right)
$$

belongs to $U_{Q}$, as $Q^{-1} X_{0}^{*} Q=X_{0}=X_{0}^{-1}$, and the corresponding isometry of $\mathbb{H}_{\mathbb{C}}^{n}$ sends $\infty \in \partial_{\infty} \mathbb{H}_{\mathbb{C}}^{n}$ to $(0,0) \in \partial_{\infty} \mathbb{H}_{\mathbb{C}}^{n}$. Hence $X_{0}$ sends the horoballs centered at $\infty$ to the horoballs centered at $(0,0)$. Let $s>0$, an easy computation shows that

$$
X_{0} \mathcal{H}_{s}=\left\{\left(w_{0}, w\right) \in \mathbb{C} \times \mathbb{C}^{n-1}: 2 \operatorname{Re} w_{0}-|w|^{2} \geq s\left|w_{0}\right|^{2}\right\} .
$$

For every $t$ in $\mathbb{R}$, the point $u_{\zeta, v} \circ c_{0}(t)$ belongs to the horosphere $X_{0} \partial \mathcal{H}_{s}$ if and only if

$$
2 \operatorname{Re}\left(e^{-t}+\left(|\zeta|^{2}-i v\right) / 2\right)-|\zeta|^{2}=s\left|e^{-t}+\left(|\zeta|^{2}-i v\right) / 2\right|^{2},
$$

that is, if and only if

$$
s e^{-2 t}+\left(s|\zeta|^{2}-2\right) e^{-t}+\frac{s}{4}\left(|\zeta|^{4}+v^{2}\right)=0 .
$$

The horoball $X_{0} \mathcal{H}_{s}$ is hence tangent to the geodesic line $u_{\zeta, v} \circ c_{0}$ if and only if the above quadratic equation with unknown $e^{-t}$ has a double solution, that is, if and only if its discriminant $\Delta$ is 0 . An easy computation gives $-\Delta=s^{2} v^{2}+4 s|\zeta|^{2}-4$. Thus, the horoball $X_{0} \mathcal{H}_{s}$ is tangent to $u_{\zeta, v} \circ c_{0}$ if and only if

$$
s=\frac{2}{\sqrt{|\zeta|^{4}+v^{2}}+|\zeta|^{2}} .
$$

As the geodesic line $c_{0}$ passes through the point at infinity of both horoballs $\mathcal{H}_{s_{0}}$ and $X_{0} \mathcal{H}_{s}$ (which have disjoint interiors by assumption), the Riemannian distance between 
them is the length of the subsegment of $c_{0}$ joining them. Note that $c_{0}$ meets $X_{0} \partial \mathcal{H}_{s}$ at $\left(\frac{2}{s}, 0\right)$. Hence, by an easy computation in $\mathbb{H}_{\mathbb{C}}^{1}$,

$$
\begin{aligned}
\ell^{\prime} & =d_{\mathbb{H}_{\mathbb{C}}^{n}}^{\prime}\left(\mathcal{H}_{s_{0}}, X_{0} \mathcal{H}_{s}\right)=\frac{1}{2} d_{\mathbb{H}_{\mathbb{C}}^{n}}\left(\mathcal{H}_{s_{0}}, X_{0} \mathcal{H}_{s}\right)=\frac{1}{2} d_{\mathbb{H}_{\mathbb{C}}^{n}}\left(\left(\frac{s_{0}}{2}, 0\right),\left(\frac{2}{s}, 0\right)\right) \\
& =\frac{1}{2}\left(\log \frac{s_{0}}{2}-\log \frac{2}{s}\right) .
\end{aligned}
$$

By Equation (44) and the definition of $d_{\text {Cyg }}^{\prime}$, the result follows.

For every $X$ in $\mathrm{U}_{Q}$, we will denote by $c=c(X)$ its $(3,1)$-coefficient in its matrix by blocks. Note that $X$ fixes $\infty$ if and only if $c=0$, by the equations (42). Equivalently, by the same set of equations, a matrix fixes $\infty$ if and only if it is upper triangular by blocks (this is the main reason why we chose the Hermitian form $q$ rather than the one in [23]). The following lemma is completely analogous to Proposition 3.14 of [31], but as we are using a different quadratic form, we need to give a proof.

Lemma 6.3 For every $X$ in $\mathrm{U}_{Q}$ and every $s>0$ such that the horoballs $\mathcal{H}_{s}$ and $X \mathcal{H}_{s}$ have disjoint interiors, we have

$$
d_{\mathbb{H}_{\mathbb{C}}^{n}}^{\prime}\left(\mathcal{H}_{s}, X \mathcal{H}_{s}\right)=\log |c|+\log \frac{s}{2}
$$

Proof As $\mathcal{H}_{s}$ and $X \mathcal{H}_{s}$ have disjoint interiors, $X$ does not fix $\infty$, hence $c \neq 0$. Left and right multiplication of $X$ by an element $u_{\zeta, v}$ for some $(\zeta, v)$ in Heis $2 n-1$ does not change the coefficient $c$ of $X$, nor does it change $d_{\mathbb{H}_{\mathbb{C}}^{n}}^{\prime}\left(\mathcal{H}_{s}, X \mathcal{H}_{s}\right)=\frac{1}{2} d_{\mathbb{H}_{\mathbb{C}}^{n}}\left(\mathcal{H}_{s}, X \mathcal{H}_{s}\right)$, as $u_{\zeta, v}$ preserves the distance $d_{\mathbb{H}_{\mathbb{C}}^{n}}$ and each horosphere centered at $\infty$. Hence, as Heis $_{2 n-1}$ acts transitively on $\partial_{\infty} \mathbb{H}_{\mathbb{C}}^{n}-\{\infty\}$, we may assume that $X \infty=(0,0)$ and that $X^{-1} \infty=(0,0)$. As $X \infty=(0,0)$, the coefficients $a, \alpha$ of $X$ are 0 , and hence by the second equation of (42), the coefficient $\gamma$ is 0 . As $X^{-1} \infty=(0,0)$, the coefficients $d, \delta$ of $X$ are 0 , and hence by the fifth equation of (42), the coefficient $\beta$ is 0 . Therefore, by the third and fourth equation of (42), the matrix $X$ has the form

$$
\left(\begin{array}{ccc}
0 & 0 & \frac{1}{\bar{c}} \\
0 & A & 0 \\
c & 0 & 0
\end{array}\right),
$$

with $A$ unitary. An easy computation, similar to the one we already did with $X_{0}$, shows that

$$
X \mathcal{H}_{s}=\left\{\left(w_{0}, w\right) \in \mathbb{C} \times \mathbb{C}^{n-1}: 2 \operatorname{Re} w_{0}-|w|^{2} \geq s|c|^{2}\left|w_{0}\right|^{2}\right\} .
$$


Hence, as above, since $s / 2 \geq 2 /\left(s|c|^{2}\right)$ as the horoballs $\mathcal{H}_{s}$ and $X \mathcal{H}_{s}$ have disjoint interiors,

$$
\begin{aligned}
d_{\mathbb{H}}^{\prime n}\left(\mathcal{H}_{s}, X \mathcal{H}_{s}\right) & =\frac{1}{2} d_{\mathbb{H}_{\mathbb{C}}^{n}}\left(\mathcal{H}_{s}, X \mathcal{H}_{s}\right) \\
& =\frac{1}{2} d_{\mathbb{H}_{\mathbb{C}}^{n}}\left(\left(\frac{s}{2}, 0\right),\left(\frac{2}{s|c|^{2}}, 0\right)\right)=\log |c|+\log \frac{s}{2}
\end{aligned}
$$

Let $m$ be a squarefree positive integer, let $K_{-m}=\mathbb{Q}(i \sqrt{m})$ be the corresponding imaginary quadratic number field, and let $\mathcal{O}_{-m}$ be the ring of integers of $K_{-m}$. An order $\mathcal{O}$ in $K_{-m}$ is a unitary subring of $\mathcal{O}_{-m}$ which is a free $\mathbb{Z}$-module of rank 2 . We use for instance [14, Chapter 7] for a general reference on these objects. An example of an order in $K_{-m}$ is $\mathbb{Z}[i \sqrt{m}]$, and $\mathcal{O}_{-m}$ is the maximal order of $K_{-m}$. In particular, $\mathcal{O}$ contains a $\mathbb{Q}$-basis of $K_{-m}$, and the field of fractions of $\mathcal{O}$ is $K_{-m}$. Let $\omega$ be an element of $\mathcal{O}$ with $\operatorname{Im} \omega>0$ such that $\mathcal{O}=\mathbb{Z}[\omega]=\mathbb{Z}+\omega \mathbb{Z}$.

As $\mathcal{O}$ is stable by complex conjugation, the subset

$$
\mathrm{SU}_{Q}(\mathcal{O})=\mathrm{SU}_{Q} \cap \mathcal{M}_{n+1}(\mathcal{O})
$$

is a discrete subgroup of the semisimple connected real Lie group $\mathrm{SU}_{Q}=\mathrm{U}_{Q} \cap$ $\mathrm{SL}_{n+1}(\mathbb{C})$.

Let $\mathcal{I}$ be a nonzero ideal of $\mathcal{O}$. We denote by $\Gamma_{\mathbb{C}, \mathcal{I}}$ the preimage, by the group morphism $\mathrm{SU}_{Q}(\mathcal{O}) \rightarrow \mathrm{SL}_{n+1}(\mathcal{O} / \mathcal{I})$ of reduction modulo $\mathcal{I}$, of the parabolic subgroup of matrices whose first column has all its coefficients 0 except the first one. As $\mathcal{O} / \mathcal{I}$ is finite ( $\mathcal{I}$ is nonzero), $\Gamma_{\mathbb{C}, \mathcal{I}}$ is a finite index subgroup of $\mathrm{SU}_{Q}(\mathcal{O})$. In particular, if $\mathcal{I}=\mathcal{O}$, then $\Gamma_{\mathbb{C}, \mathcal{I}}=\Gamma_{\mathbb{C}, \mathcal{O}}=\operatorname{SU}_{Q}(\mathcal{O})$.

Recall that a horoball $H$ centered at a point $\xi$ in a CAT(-1) metric space $X$ is precisely invariant under a group of isometries $\Gamma$ if for every $g \in \Gamma$ that does not fix $\xi$, the intersection $g \stackrel{\circ}{H} \cap \stackrel{\circ}{H}$ is empty.

Lemma 6.4 For $v=2 \operatorname{Im} \omega$ if $\operatorname{Re} \omega \in \mathbb{Z}$, and $v=4 \operatorname{Im} \omega$ otherwise, the horoball $\mathcal{H}_{v}$ is precisely invariant under $\Gamma_{\mathbb{C}, \mathcal{I}}$. Furthermore, if $\mathcal{I}=\mathcal{O}=\mathcal{O}_{-1}$, then $\mathcal{H}_{2}$ is the maximal horoball centered at $\infty$ which is precisely invariant under $\Gamma_{\mathbb{C}, \mathcal{I}}$.

Proof With $v$ as in the statement, the element $-i v / 2$ belongs to $\mathcal{O}$, as $i \operatorname{Im} \omega=$ $\omega-\operatorname{Re} \omega$ belongs to $\mathcal{O}$ if $\operatorname{Re} \omega \in \mathbb{Z}$, and $2 i \operatorname{Im} \omega=\omega-\bar{\omega}$ belongs to $\mathcal{O}$ (which is stable by conjugation). Hence $u_{0, v}$ belongs to $\Gamma_{\mathbb{C}, \mathcal{I}}$. It follows for instance from Hersonsky and Paulin [28, Proposition 5.7] (which is an easy consequence of the complex hyperbolic Shimizu inequality of Kamiya [37] and Parker [46]) that the 
horoball $\mathcal{H}_{v}$ is precisely invariant (the Hermitian form $q$ in [28] is not the same one as the one above, but it is equivalent by a permutation of coordinates, hence we may indeed apply Proposition 5.7 of [28]).

If $\mathcal{I}=\mathcal{O}=\mathcal{O}_{-1}$, then $X_{0}$ defined in Equation (43) belongs to $\Gamma_{\mathbb{C}, \mathcal{I}}$ and $\operatorname{Im} \omega=1$, $\operatorname{Re} \omega \in \mathbb{Z}$. By Lemma 6.3, we have $d\left(\mathcal{H}_{2}, X_{0} \mathcal{H}_{2}\right)=0$, hence the last assertion follows.

For every $(a, \alpha, c) \in \mathcal{O} \times \mathcal{O}^{n-1} \times \mathcal{O}$, let $\langle a, \alpha, c\rangle$ be the ideal of $\mathcal{O}$ generated by $a, c$ and the components of $\alpha$.

Proposition 6.5 If $n=2$ and $\mathcal{O}=\mathcal{O}_{-m}$, then

(1) for every $\mathcal{I}$, the set of parabolic fixed points of $\Gamma_{\mathbb{C}, \mathcal{I}}$ is exactly the set of points in $\partial_{\infty} \mathbb{H}_{\mathbb{C}}^{n}$ having homogeneous coordinates in $\mathbb{P}_{n}(\mathbb{C})$ that are elements in $K_{-m}$,

(2) the orbit $\Gamma_{\mathbb{C}, \mathcal{I}} \cdot \infty$ is exactly the set of points in $\partial_{\infty} \mathbb{H}_{\mathbb{C}}^{n}$ having homogeneous coordinates in $\mathbb{P}_{n}(\mathbb{C})$ of the form $[a: \alpha: c]$ with $(a, \alpha, c) \in \mathcal{O} \times \mathcal{I}^{n-1} \times \mathcal{I}$, $2 \operatorname{Re} a \bar{c}=|\alpha|^{2}$ and $\langle a, \alpha, c\rangle=\mathcal{O}$,

(3) if $m=1,2,3,7,11,19,43,67,163$ and $\mathcal{I}=\mathcal{O}$, then $\Gamma_{\mathbb{C}, \mathcal{I}}$ has only one orbit of parabolic fixed points.

Proof (1) If $\mathcal{I}=\mathcal{O}$, then the first result is due to Holzapfel [35; 36, page 280]. As $\Gamma_{\mathbb{C}, \mathcal{I}}$ has finite index in $\mathrm{SU}_{Q}(\mathcal{O})$, and as a discrete group and a finite index subgroup have the same set of parabolic fixed points, the first claim follows.

(2) A result of Feustel [19] (see Holzapfel [36, page 280] and Zink [63]) says that the map which associates to a parabolic fixed point of $\mathrm{SU}_{Q}(\mathcal{O})$ the fractional ideal generated by its homogeneous coordinates in $\mathcal{O}_{-m}$ induces a bijection from the set of orbits under $\mathrm{SU}_{Q}(\mathcal{O})$ of parabolic fixed points of $\mathrm{SU}_{Q}(\mathcal{O})$ to the set of ideal classes of $K_{-m}$. As $\infty$ corresponds to [1:0:0] whose coordinates generate the trivial fractional ideal, and as $K_{-m}$ has class number one if and only if $m=1,2,3,7,11,19,43,67,163$ (see Cox [14, Theorem 7.30]), the second claim follows if $\mathcal{I}=\mathcal{O}$, as well as claim (3). If $M \in \Gamma_{\mathbb{C}, \mathcal{I}}$ and

then

$$
M\left(\begin{array}{l}
1 \\
0 \\
0
\end{array}\right)=\left(\begin{array}{l}
a \\
\alpha \\
c
\end{array}\right),
$$

$$
\left(\begin{array}{l}
a \\
\alpha \\
c
\end{array}\right)
$$

is the first column of the matrix $M$, so that the second claim if $\mathcal{I} \neq \mathcal{O}$ follows by the definition of $\Gamma_{\mathbb{C}, \mathcal{I}}$. 


\subsection{Quaternions and 5-dimensional real hyperbolic geometry}

Let $\mathbb{H}$ be Hamilton's quaternion algebra over $\mathbb{R}$, generated as a real vector space by the standard basis $1, i, j, k$, with products $k=i j=-j i, i^{2}=-1, j^{2}=-1$ and unit 1 . Recall that the conjugate of the quaternion $z=x_{1}+x_{2} i+x_{3} j+x_{4} k$ is $\bar{z}=x_{1}-x_{2} i-x_{3} j-x_{4} k$, which satisfies $\overline{z w}=\bar{w} \bar{z}$, and that the absolute value of $z$ (or the square root of its reduced norm) is

$$
|z|=\sqrt{N(z)}=\sqrt{z \bar{z}}=\sqrt{\bar{z} z}=\sqrt{x_{1}^{2}+x_{2}^{2}+x_{3}^{2}+x_{4}^{2}} .
$$

The Dieudonné determinant (see Dieudonné [17] and Aslaksen [4]) $\Delta$ is the group morphism from the group $\mathrm{GL}_{2}(\mathbb{H})$ of invertible $2 \times 2$ matrices with coefficients in $\mathbb{H}$ to $\mathbb{R}_{+}^{*}$, given by

$$
\Delta\left(\left(\begin{array}{ll}
a & b \\
c & d
\end{array}\right)\right)= \begin{cases}\left|a d-a c a^{-1} b\right| & \text { if } a \neq 0 \\
\left|c b-c a c^{-1} d\right| & \text { if } c \neq 0\end{cases}
$$

We will denote by $\mathrm{SL}_{2}(\mathbb{H})$ the group of $2 \times 2$ quaternionic matrices with Dieudonné determinant 1 . We refer for instance to Kellerhals [38] for more information on $\mathrm{SL}_{2}(\mathbb{H})$. Note that this notation is different from, hence should not be confused with, the notation $\operatorname{SL}\left(2, C_{n}\right)$ for $n=3$ and $C_{3}=\mathbb{H}$ of Vahlen and Ahlfors [2] (see also Maclachlan, Waterman and Wielenberg [43]), giving a description of the isometry group of the real hyperbolic $(n+1)$-space using the $2^{n}$-dimensional real Clifford algebra $C_{n}$.

The group $\mathrm{SL}_{2}(\mathbb{H})$ acts on the Alexandrov compactification $\mathbb{H} \cup\{\infty\}$ of $\mathbb{H}$ by

$$
\left(\begin{array}{ll}
a & b \\
c & d
\end{array}\right) \cdot z= \begin{cases}(a z+b)(c z+d)^{-1} & \text { if } z \neq \infty,-c^{-1} d \\
a c^{-1} & \text { if } z=\infty, c \neq 0 \\
\infty & \text { otherwise }\end{cases}
$$

It is well known (see for instance Kellerhals [38]) that $\mathrm{PSL}_{2}(\mathbb{H})=\mathrm{SL}_{2}(\mathbb{H}) /\{ \pm \mathrm{Id}\}$ is the orientation preserving conformal group of the 4 -sphere $\mathbb{H} \cup\{\infty\}$ with its standard conformal structure defined by the 4 -dimensional Euclidean space $(\mathbb{H},|\cdot|)$. In the upper halfspace model $\mathbb{H}_{\mathbb{R}}^{5}$ of the 5-dimensional real hyperbolic space with constant curvature -1 , consider the coordinates $(z, t)$ with $z \in \mathbb{H}$ and $t>0$ (called the vertical coordinate), so that $\partial_{\infty} \mathbb{H}_{\mathbb{R}}^{5}$ identifies with the union of $\mathbb{H}$ (for $t=0$ ) and of $\{\infty\}$. By the Poincaré extension procedure (see for instance Beardon [5, Section 3.3]), the group $\mathrm{PSL}_{2}(\mathbb{H})$ hence identifies with the group of orientation preserving isometries of $\mathbb{H}_{\mathbb{R}}^{5}$. We will denote the Riemannian distance on $\mathbb{H}_{\mathbb{R}}^{5}$ by $d_{\mathbb{H}_{\mathbb{R}}^{5}}^{\prime}$. 
Lemma 6.6 [27, Theorem 1.- 2)] For every

$$
g=\left(\begin{array}{ll}
a & b \\
c & d
\end{array}\right)
$$

in $\mathrm{SL}_{2}(\mathbb{H})$, and $(z, t)$ in $\mathbb{H}_{\mathbb{R}}^{5}$, the vertical coordinate of $g(z, t)$ is

$$
\frac{t}{|c z+d|^{2}+|c|^{2} t^{2}} \text {. }
$$

Proof As [27] is an announcement, we give a proof for the sake of completeness. The proof is an adaptation of the proof for $\mathrm{SL}_{2}(\mathbb{C})$ in $[5$, page 58], the main problem consists of being careful with the noncommutativity of $\mathbb{H}$. We may assume that $c \neq 0$, as the map $z \mapsto \alpha z \beta+\gamma$ for $\alpha, \beta, \gamma$ in $\mathbb{H}^{*} \times \mathbb{H}^{*} \times \mathbb{H}$ is a Euclidean similitude of ratio $|\alpha \beta|$. Define the isometric sphere of $g$ to be the sphere $S_{g}$ of center $-c^{-1} d$ and radius $1 /|c|$ in the Euclidean space $(\mathbb{H},|\cdot|)$. By the definition of a Euclidean reflection with respect to a sphere in this Euclidean space, the map

$$
\sigma: z \mapsto-c^{-1} d+\frac{1}{|c|^{2}} \frac{z+c^{-1} d}{\left|z+c^{-1} d\right|^{2}}
$$

is the Euclidean reflection with respect to the sphere $S_{g}$. An easy computation shows that the map $\varphi=g \circ \sigma$ is

$$
z \mapsto\left(b-a c^{-1} d\right)(\bar{z} \bar{c}+\bar{d})+a c^{-1},
$$

which is a Euclidean isometry, as $z \mapsto \bar{z}$ is, and $\left|c b-c a c^{-1} d\right|=1$. The Poincaré extension of $\varphi$ preserves the vertical coordinates, and the Poincaré extension of $\sigma$ is the Euclidean reflection with respect to the sphere in $\mathbb{H}_{\mathbb{R}}^{5}$ whose equator is $S_{g}$. As $g=\varphi \circ \sigma$, the result follows by an easy computation.

The horoballs centered at $\infty$ in $\mathbb{H}_{\mathbb{R}}^{5}$ are the subsets $\mathcal{H}_{s}$ for $s>0$, where

$$
\mathcal{H}_{s}=\{(z, t) \in \mathbb{H} \times] 0,+\infty[: t \geq s\} .
$$

Lemma 6.7 For every

$$
g=\left(\begin{array}{ll}
a & b \\
c & d
\end{array}\right)
$$

in $\mathrm{SL}_{2}(\mathbb{H})$, and every $s>0$ such that the horoballs $\mathcal{H}_{s}$ and $g \mathcal{H}_{s}$ have disjoint interiors, we have

$$
d\left(\mathcal{H}_{s}, g \mathcal{H}_{s}\right)=2 \log |c|+2 \log s
$$


Proof $A s \mathcal{H}_{s}$ and $g \mathcal{H}_{s}$ have disjoint interiors, we have $c \neq 0$. The map $g$ sends the geodesic line between $-c^{-1} d$ and $\infty$ to the geodesic line between $\infty$ and $a c^{-1}$, hence the point $\left(-c^{-1} d, s\right)$ of intersection of the first line with $\mathcal{H}_{s}$ is sent to $g\left(-c^{-1} d, s\right)$, which is the point of intersection of the second line with $g \mathcal{H}_{s}$. The vertical coordinate of $g\left(-c^{-1} d, s\right)$ is $1 /\left(|c|^{2} s\right)$ by the previous Lemma 6.6. Hence the result follows by an easy computation of hyperbolic distances.

We will use Vignéras [61] and Maclachlan and Reid [42, Section 2] as general references on quaternion algebras. Let $A(\mathbb{Q})$ be a quaternion algebra over $\mathbb{Q}$, which is ramified over $\mathbb{R}$, that is, the real algebra $A(\mathbb{Q}) \otimes \mathbb{Q} \mathbb{R}$ is isomorphic to Hamilton's algebra $\mathbb{H}$. We identify $A(\mathbb{Q}) \otimes_{\mathbb{Q}} \mathbb{R}$ and $\mathbb{H}$ by any such isomorphism. Let $\mathcal{O}^{\prime}$ be an order of $A(\mathbb{Q})$, that is an unitary subring which is a finitely generated $\mathbb{Z}$-module generating the $\mathbb{Q}$-vector space $A(\mathbb{Q})$. For instance, if

$$
A(\mathbb{Q})=\left\{x_{1}+x_{2} i+x_{3} j+x_{4} k \in \mathbb{H}: x_{1}, x_{2}, x_{3}, x_{4} \in \mathbb{Q}\right\},
$$

we can take

$$
\mathcal{O}^{\prime}=\left\{x_{1}+x_{2} i+x_{3} j+x_{4} k \in \mathbb{H}: x_{1}, x_{2}, x_{3}, x_{4} \in \mathbb{Z}\right\},
$$

or the Hurwitz ring

$$
\mathcal{O}^{\prime}=\left\{x_{1} \frac{1+i+j+k}{2}+x_{2} i+x_{3} j+x_{4} k \in \mathbb{H}: x_{1}, x_{2}, x_{3}, x_{4} \in \mathbb{Z}\right\},
$$

which is a maximal order. Let $\mathcal{I}^{\prime}$ be a nonzero two-sided ideal in the ring $\mathcal{O}^{\prime}$.

We denote by $\Gamma_{\mathcal{I}^{\prime}}$ the preimage in the group morphism $\mathrm{SL}_{2}\left(\mathcal{O}^{\prime}\right) \rightarrow \mathrm{GL}_{2}\left(\mathcal{O}^{\prime} / \mathcal{I}^{\prime}\right)$ of reduction modulo $\mathcal{I}^{\prime}$ of the subgroup of upper triangular matrices. As $\mathcal{O}^{\prime} / \mathcal{I}^{\prime}$ is finite ( $\mathcal{I}^{\prime}$ is nonzero), $\Gamma_{\mathcal{I}^{\prime}}$ is a finite index subgroup of $\mathrm{SL}_{2}\left(\mathcal{O}^{\prime}\right)$.

Lemma 6.8 The horoball $\mathcal{H}_{1}$ is precisely invariant under $\Gamma_{\mathcal{I}^{\prime}}$. Furthermore, if $\mathcal{I}^{\prime}=$ $\mathcal{O}^{\prime}$, then $\mathcal{H}_{1}$ is the maximal horoball centered at $\infty$ which is precisely invariant under $\Gamma_{\mathcal{I}^{\prime}}$.

Proof The element

$$
\left(\begin{array}{ll}
1 & 1 \\
0 & 1
\end{array}\right)
$$

belongs to $\Gamma_{\mathcal{I}^{\prime}}$. It follows from Kellerhals [38, page 1091] that the horoball $\mathcal{H}_{1}$ is precisely invariant.

If $\mathcal{I}^{\prime}=\mathcal{O}^{\prime}$, then

$$
g=\left(\begin{array}{cc}
0 & 1 \\
-1 & 0
\end{array}\right)
$$

belongs to $\Gamma_{\mathcal{I}^{\prime}}$, and by Lemma $6.7, d\left(\mathcal{H}_{1}, g \mathcal{H}_{1}\right)=0$, so the last assertion follows. 


\subsection{On arithmetic lattices}

The following result follows from the work of Borel and Harish-Chandra [8] and of Borel [7, Theorem 1.10] (see Borel [6] for an elementary presentation of semisimple algebraic groups). Two subgroups $A$ and $B$ of a group $C$ are said to be commensurable in this theorem if $A \cap B$ has finite index in both $A$ and $B$.

Theorem 6.9 [8; 7] Let $\underline{G}$ be a connected semisimple algebraic group defined over $\mathbb{Q}$ of $\mathbb{R}$-rank one and $\underline{P}$ be a minimal parabolic subgroup of $\underline{G}$ defined over $\mathbb{Q}$, let $G=\underline{G}(\mathbb{R})_{0}$ and $P=G \cap \underline{P}(\mathbb{R})$, let $\Gamma$ be a subgroup of $G$ commensurable to $\underline{G}(\mathbb{Z}) \cap G$, then $\Gamma$ is a lattice in $G$, and the set of parabolic fixed points of $\Gamma$ on $G / P$ is $\underline{G}(\mathbb{Q}) P$.

Such a subgroup $\Gamma$ will be called an arithmetic lattice in $G$. Note that the $\mathbb{R}$-rank assumption is equivalent to the fact that for every (or equivalently any) maximal compact subgroup $K$ of the Lie group $G$, the associated symmetric space $X=G / K$ may be endowed with a $G$-invariant Riemannian metric with sectional curvature at most -1 . Such a metric is then unique up to multiplication by a positive constant, and $P$ is the stabilizer of a point in the boundary at infinity $\partial_{\infty} X$. The orbital map at this point hence induces a $G$-equivariant homeomorphism between $G / P$ and $\partial_{\infty} X$. Note that there is a terminology problem: by a parabolic element, we mean an isometry of $X$ having a unique fixed point (called a parabolic fixed point) on $X \cup \partial_{\infty} X$, that belongs to $\partial_{\infty} X$, but the set of real points of a parabolic subgroup of $\underline{G}$ also contains nonparabolic elements!

Examples In (1) and (2) below, let $m$ be a squarefree positive integer, and let $\mathcal{I}$ be a nonzero ideal in an order $\mathcal{O}$ in the ring of integers $\mathcal{O}_{-m}$ of the imaginary quadratic number field $K_{-m}=\mathbb{Q}(i \sqrt{m})$. Let $(1, \omega)$ be a basis of $\mathcal{O}$ as a $\mathbb{Z}$-module. It is also a basis of $K_{-m}$ as a $\mathbb{Q}$-vector space, and of $\mathbb{C}$ as an $\mathbb{R}$-vector space. If $x_{1}, \ldots, x_{n}, y_{1}, \ldots, y_{n}$ are real numbers, as $\omega$ is a quadratic integer, note that

$$
\prod_{i=1}^{n}\left(x_{i}+\omega y_{i}\right)=P\left(x_{1}, \ldots, x_{n}, y_{1}, \ldots, y_{n}\right)+\omega Q\left(x_{1}, \ldots, x_{n}, y_{1}, \ldots, y_{n}\right)
$$

where $P$ and $Q$ are polynomials in $x_{1}, \ldots, x_{n}, y_{1}, \ldots, y_{n}$ with integer coefficients.

(1) By writing each coefficient of a $n \times n$ complex matrix $X$ in the basis $(1, \omega)$ over $\mathbb{R}$, the equation det $X=1$ gives a system of two polynomial equations with integer coefficients, with unknown the coordinates of the coefficients of $X$ in $(1, \omega)$.

Hence, there exists an algebraic group $\underline{G}$ defined over $\mathbb{Q}$ such that $\underline{G}(\mathbb{Z})=\mathrm{SL}_{2}(\mathcal{O})$, $\underline{G}(\mathbb{Q})=\mathrm{SL}_{2}\left(K_{-m}\right)$ and $\underline{G}(\mathbb{R})=\mathrm{SL}_{2}(\mathbb{C})$. As the Lie group $\underline{G}(\mathbb{R})$ is connected and 
semisimple, with associated symmetric space the real hyperbolic 3-space, the algebraic group $\underline{G}$ is connected, semisimple with $\mathbb{R}$-rank one. Let $\underline{P}$ be the algebraic subgroup of $\underline{G}$ corresponding to the upper triangular subgroup of $2 \times 2$ matrices, so that $P$ is the stabilizer of the point at infinity $\infty$ in the upper halfspace model of $\mathbb{H}_{\mathbb{R}}^{3}$.

Let $\Gamma_{\mathbb{R}, \mathcal{I}}$ be the finite index subgroup of the group $\mathrm{SL}_{2}(\mathcal{O})$, which is the preimage, by the group morphism $\mathrm{SL}_{2}(\mathcal{O}) \rightarrow \mathrm{SL}_{2}(\mathcal{O} / \mathcal{I})$ of reduction modulo $\mathcal{I}$, of the subgroup of upper triangular matrices. By Theorem 6.9, the subgroup $\Gamma_{\mathbb{R}, \mathcal{I}}$ is a lattice in $\mathrm{SL}_{2}(\mathbb{C})$, and its set of parabolic fixed points is

as

$$
\mathcal{P}_{\Gamma_{\mathbb{R}, \mathcal{I}}}=\mathrm{SL}_{2}\left(K_{-m}\right) \cdot \infty=K_{-m} \cup\{\infty\},
$$$$
\left(\begin{array}{cc}
0 & -1 \\
1 & 0
\end{array}\right) \text { and }\left(\begin{array}{cc}
x & 0 \\
1 & x^{-1}
\end{array}\right) \text {, }
$$

for every $x$ in $K_{-m}-\{0\}$, are elements of $\mathrm{SL}_{2}\left(K_{-m}\right)$ sending $\infty$ to 0 and $x$ respectively.

Note that if $\mathcal{I}=\mathcal{O}=\mathcal{O}_{-m}$, then $\Gamma_{\mathbb{R}, \mathcal{I}}=\operatorname{PSL}_{2}\left(\mathcal{O}_{-m}\right)$ is a Bianchi group, which is well-known to be a lattice in $\mathrm{PSL}_{2}(\mathbb{C})$ (see for instance Maclachlan and Reid [42]). The fact that $\mathcal{P}_{\Gamma_{\mathbb{R}, \mathcal{O}_{-m}}}=K_{-m} \cup\{\infty\}$ is also proven in Elstrodt, Grunewald and Mennicke [18, Proposition 2.2, page 314].

(2) Recall that $N(\omega)=\omega \bar{\omega}$ and $\operatorname{Tr}(\omega)=\omega+\bar{\omega}=2 \operatorname{Re} \omega$ are integers, as $\omega$ is an algebraic integer. If $x, y, x^{\prime}, y^{\prime}$ are real numbers, note that

$$
(x+\bar{\omega} y)\left(x^{\prime}+\omega y^{\prime}\right)=\left(x x^{\prime}+N(\omega) y y^{\prime}+\operatorname{Tr}(\omega) y x^{\prime}\right)+\omega\left(x y^{\prime}-y x^{\prime}\right) .
$$

Recall that the matrix $Q$ (introduced in Equation (41)) has integer coefficients. Hence by writing each coefficient of a $(n+1) \times(n+1)$ complex matrix $X$ in the basis $(1, \omega)$ over $\mathbb{R}$, the system of equations given by $\operatorname{det} X=1$ and $X^{*} Q X=Q$ becomes a system of $2\left((n+1)^{2}+1\right)$ polynomial equations with integer coefficients, with unknown the coordinates of the coefficients of $X$ in $(1, \omega)$.

Therefore there exists an algebraic group $\underline{G}$ defined over $\mathbb{Q}$ such that $\underline{G}(\mathbb{Z})=\operatorname{SU}_{Q}(\mathcal{O})$, $\underline{G}(\mathbb{Q})=\mathrm{SU}_{Q}\left(K_{-m}\right)$ and $\underline{G}(\mathbb{R})=\mathrm{SU}_{Q}$. As the Lie group $\underline{G}(\mathbb{R})$ is connected and semisimple, with associated symmetric space the complex hyperbolic $n$-space, the algebraic group $\underline{G}$ is connected, semisimple with $\mathbb{R}$-rank one. Let $\underline{P}$ be the algebraic subgroup of $\underline{G}$ corresponding to the upper triangular by blocks subgroup of $(n+1) \times$ $(n+1)$ matrices, so that $P$ is the stabilizer of the point at infinity $\infty$ in the Siegel domain model of $\mathbb{H}_{\mathbb{C}}^{n}$, or of the point $[1: 0: 0]$ in the projective model.

By Theorem 6.9, the group $\Gamma_{\mathbb{C}, \mathcal{I}}$ defined in Section 6.1 is a lattice in $\mathrm{SU}_{Q}$, and its set of parabolic fixed points is $\mathcal{P}_{\Gamma_{\mathbb{C}, \mathcal{I}}}=\mathrm{SU}_{Q}\left(K_{-m}\right) \cdot \infty$. By Witt's theorem, $\mathrm{SU}_{Q}\left(K_{-m}\right)$ 
acts transitively on the isotropic lines in $K_{-m}{ }^{n+1}$ for the Hermitian form $q$. Hence $\mathcal{P}_{\Gamma_{\mathbb{C}, \mathcal{I}}}=\left\{\left[z_{0}: z: z_{n}\right] \in \mathbb{P}_{n}\left(K_{-m}\right): q\left(z_{0}, z, z_{n}\right)=0\right\}$. If $n=2$ and $\mathcal{O}=\mathcal{O}_{-m}$, we recover Proposition 6.5 (1).

(3) Let $\mathcal{I}^{\prime}$ be a nonzero two-sided ideal in an order $\mathcal{O}^{\prime}$ of a quaternion algebra $A(\mathbb{Q})$ over $\mathbb{Q}$ such that $A(\mathbb{Q}) \otimes \mathbb{Q} \mathbb{R}=\mathbb{H}$. For every field $K$ containing $\mathbb{Q}$, define $A(K)=A(\mathbb{Q}) \otimes \mathbb{Q} K$. Let $\left(e_{1}, e_{2}, e_{3}, e_{4}\right)$ be a basis of $\mathcal{O}^{\prime}$ as a $\mathbb{Z}$-module. It is also a basis of $A(K)$ as a $K$-vector space for every field $K$ containing $\mathbb{Q}$.

If $x=x_{1}+x_{2} i+x_{3} j+x_{4} k$ is an element in $\mathbb{H}$, written in the standard basis $(1, i, j, k)$, let $\operatorname{Tr} x=2 x_{1}$ be its reduced trace, and $N(x)=x_{1}^{2}+x_{2}^{2}+x_{3}^{2}+x_{4}^{2}$ be its reduced norm. A $2 \times 2$ matrix

$$
X=\left(\begin{array}{ll}
a & b \\
c & d
\end{array}\right)
$$

with coefficients in $\mathbb{H}$ has Dieudonné determinant 1 if and only if

$$
N(a d)+N(b c)-\operatorname{Tr}(a \bar{c} d \bar{b})=1
$$

(see for instance Kellerhals [38, page 1085]). The maps $\mathbb{R}^{4} \rightarrow \mathbb{R}$ defined by sending $\left(x_{1}, x_{2}, x_{3}, x_{4}\right)$ to $N\left(x_{1} e_{1}+x_{2} e_{2}+x_{3} e_{3}+x_{4} e_{4}\right)$ and $\operatorname{Tr}\left(x_{1} e_{1}+x_{2} e_{2}+x_{3} e_{3}+x_{4} e_{4}\right)$ are polynomial maps in $x_{1}, x_{2}, x_{3}, x_{4}$ with rational coefficients.

By writing each coefficient $a, b, c, d$ of a $2 \times 2$ matrix $X$ with coefficients in $A(K)$ in the basis $\left(e_{1}, e_{2}, e_{3}, e_{4}\right)$ for any field $K$, Equation (45) becomes a polynomial equation with coefficients in $\mathbb{Q}$, with unknown the coordinates of the coefficients of $X$ in $\left(e_{1}, e_{2}, e_{3}, e_{4}\right)$.

Hence there exists an algebraic group $\underline{G}$ defined over $\mathbb{Q}$ such that $\underline{G}(\mathbb{Z})=\operatorname{SL}_{2}\left(\mathcal{O}^{\prime}\right)$ and $\underline{G}(K)=\mathrm{SL}_{2}(A(K))$ for every field $K$ containing $\mathbb{Q}$. As the Lie group $\underline{G}(\mathbb{R})=$ $\mathrm{SL}_{2}(\mathbb{H})$ is connected and semisimple, with associated symmetric space the real hyperbolic 5-space, the algebraic group $\underline{G}$ is connected, semisimple with $\mathbb{R}$-rank one. Let $\underline{P}$ be the algebraic subgroup of $\underline{G}$ corresponding to the upper triangular matrices, so that $P$ is the stabilizer of the point at infinity $\infty$ in the upper halfspace model of $\mathbb{H}_{\mathbb{R}}^{5}$.

Let $\Gamma_{\mathcal{I}^{\prime}}$ be the group introduced in Section 6.2, which has finite index in $\operatorname{SL}_{2}\left(\mathcal{O}^{\prime}\right)$. By Theorem 6.9, the subgroup $\Gamma_{\mathcal{I}^{\prime}}$ is a lattice in $\mathrm{SL}_{2}(\mathbb{H})$, and its set of parabolic fixed points is $\mathcal{P}_{\Gamma_{\mathcal{I}^{\prime}}}=\mathrm{SL}_{2}(A(\mathbb{Q})) \cdot \infty$. As $A(\mathbb{Q})$ is a division algebra, the same argument as for example (1) shows that $\mathcal{P}_{\Gamma_{\mathcal{I}^{\prime}}}=A(\mathbb{Q}) \cup\{\infty\}$.

\subsection{The ubiquity of Hall rays}

In this subsection, we give applications of our geometric results from Section 5 to the framework of Diophantine approximation in negatively curved manifolds, introduced 
in Hersonsky and Paulin [30; 31], to which we refer for notation and background. In particular, we will consider arithmetically defined examples. See also the previous works of Ford [20] and Series [57], among many others.

Let $M$ be a complete, nonelementary, geometrically finite Riemannian manifold with sectional curvature at most -1 and dimension at least 3 . Let $\pi: \widetilde{M} \rightarrow M$ be a universal Riemannian covering, with covering group $\Gamma$. Let $e$ be a cusp of $M$, and, as in Section 5.1, let $V_{e}$ be a fixed Margulis neighbourhood of $e, H_{e}$ a horoball in $\widetilde{M}$ with $\pi\left(H_{e}\right)=V_{e}$ and $\xi_{e}$ the point at infinity of $H_{e}$. Note that $V_{e}$ is a Margulis neighbourhood of $e$ if $H_{e}$ is precisely invariant under $\Gamma$. In the previous works [30; 31], it was required that $V_{e}$ is the maximal Margulis neighbourhood, as this makes the constructions independent of the choice of $V_{e}$. But since it is not always easy to determine the maximal Margulis neighbourhood of a cusp, and as it is not necessary for the statements, we will fix some choice of $V_{e}$ (or equivalently $H_{e}$ ) which is not necessarily maximal.

Three (classes of) examples Many of these examples are in fact orbifolds rather than manifolds, but the extension to this context is obvious. We use the same notation as in the examples of Section 6.3.

(1) Let $\Gamma_{\mathbb{R}, \mathcal{I}}$ be the finite index subgroup of the group $\mathrm{SL}_{2}(\mathcal{O})$, which is the preimage, by the group morphism $\mathrm{SL}_{2}(\mathcal{O}) \rightarrow \mathrm{SL}_{2}(\mathcal{O} / \mathcal{I})$ of reduction modulo $\mathcal{I}$, of the subgroup of upper triangular matrices. The quotient $M=\Gamma_{\mathbb{R}, \mathcal{I}} \backslash \mathbb{H}_{\mathbb{R}}^{3}$ is a finite volume real hyperbolic orbifold. Let $\pi: \mathbb{H}_{\mathbb{R}}^{3} \rightarrow M$ be the canonical projection, $e$ the cusp of $M$ corresponding to $\xi_{e}=\infty$, and let $H_{e}$ be the horoball of points of Euclidean height at least 1 . As

$$
\left(\begin{array}{ll}
1 & 1 \\
0 & 1
\end{array}\right)
$$

belongs to $\Gamma_{\mathbb{R}, \mathcal{I}}$, it is well known that $H_{e}$ is precisely invariant under $\Gamma_{\mathbb{R}, \mathcal{I}}$. Furthermore, if $\mathcal{I}=\mathcal{O}$, then

$$
\left(\begin{array}{cc}
0 & 1 \\
-1 & 0
\end{array}\right)
$$

belongs to $\Gamma_{\mathbb{R}, \mathcal{I}}$, hence $H_{e}$ is maximal. For more details, see the end of Section 5 of [30].

(2) Let $\Gamma_{\mathbb{C}, \mathcal{I}}$ be the finite index subgroup of $\operatorname{SU}_{Q}(\mathcal{O})$ introduced in Section 6.1, that acts by isometries on the Siegel domain model $\mathbb{H}_{\mathbb{C}}^{n}$ of the complex hyperbolic $n$-space with (constant) holomorphic sectional curvature -1 . Let $M$ be the finite volume complex hyperbolic orbifold $\Gamma_{\mathbb{C}, \mathcal{I}} \backslash \mathbb{H}_{\mathbb{C}}^{n}$, which is endowed with the quotient of the renormalized Riemannian distance $d_{\mathbb{H}_{\mathbb{C}}^{n}}^{\prime}$ in order for its sectional curvatures to be at most -1 . Let $\pi: \mathbb{H}_{\mathbb{C}}^{n} \rightarrow M$ be the canonical projection, $e$ be the cusp of $M$ 
corresponding to $\xi_{e}=\infty$, and let $H_{e}$ be the horoball $\mathcal{H}_{2} \operatorname{Im} \omega$ if $\operatorname{Re} \omega \in \mathbb{Z}$, and $\mathcal{H}_{4} \operatorname{Im} \omega$ otherwise, which is precisely invariant under $\Gamma_{\mathbb{C}, \mathcal{I}}$ by Lemma 6.4 (and maximal if $\left.\mathcal{I}=\mathcal{O}=\mathcal{O}_{-1}\right)$.

(3) Let $\mathcal{I}^{\prime}$ be a nonzero two-sided ideal in an order $\mathcal{O}^{\prime}$ of a quaternion algebra $A(\mathbb{Q})$ over $\mathbb{Q}$ such that $A(\mathbb{Q}) \otimes_{\mathbb{Q}} \mathbb{R}=\mathbb{H}$, and $\Gamma_{\mathcal{I}^{\prime}}$ be the finite index subgroup of $\operatorname{SL}_{2}\left(\mathcal{O}^{\prime}\right)$ introduced in Section 6.2, that acts by isometries on the upper halfspace model $\mathbb{H}_{\mathbb{R}}^{5}$ of the real hyperbolic 5-space with (constant) sectional curvature -1 . Let $M$ be the finite volume real hyperbolic orbifold $\Gamma_{\mathcal{I}^{\prime}} \backslash \mathbb{H}_{\mathbb{R}}^{5}, \pi: \mathbb{H}_{\mathbb{R}}^{5} \rightarrow M$ the canonical projection, $e$ the cusp of $M$ corresponding to $\xi_{e}=\infty$, and let $H_{e}$ be the horoball $\mathcal{H}_{1}$, which is precisely invariant under $\Gamma_{\mathcal{I}^{\prime}}$ by Lemma 6.8 (and maximal if $\mathcal{I}^{\prime}=\mathcal{O}^{\prime}$ ).

Let the link of $e$ in $M, \mathrm{Lk}_{e}=\mathrm{Lk}_{e}(M)$, be the space of locally geodesic lines (up to translation at the source) starting from $e$ in $M$ that are nonwandering (in other words, such that each of them accumulates in some compact subset of $M$ ). Let Rat $e$ be the space of locally geodesic lines starting from $e$ and converging to $e$. Normalize the locally geodesic lines in $\mathrm{Lk}_{e} \cup \mathrm{Rat}_{e}$ so that their first intersection with $\partial V_{e}$ is at time 0 . Endow $\mathrm{Lk}_{e} \cup \mathrm{Rat}_{e}$ with the compact-open topology. Let $\Lambda \Gamma \subset \partial_{\infty} \widetilde{M}$ be the limit set of $\Gamma, \mathcal{P}_{\Gamma} \subset \Lambda \Gamma$ the set of parabolic fixed points of $\Gamma$, and let $\Gamma_{\infty}$ be the stabilizer of $\xi_{e}$ in $\Gamma$. Then the maps $\Gamma \xi_{e}-\left\{\xi_{e}\right\} \rightarrow$ Rat $_{e}$ and $\Lambda \Gamma-\mathcal{P}_{\Gamma} \rightarrow \mathrm{Lk}_{e}$, which associate to $x$ the projection in $M$ by $\pi$ of the geodesic line starting from $\xi_{e}$ and ending at $x$, induce a bijection $\Gamma_{\infty} \backslash\left(\Gamma \xi_{e}-\left\{\xi_{e}\right\}\right) \rightarrow$ Rat $_{e}$ and a homeomorphism

$$
\Gamma_{\infty} \backslash\left(\Lambda \Gamma-\mathcal{P}_{\Gamma}\right) \rightarrow \mathrm{Lk}_{e}
$$

We identify these spaces by these maps. Note that $\mathrm{Lk}_{e} \cup \mathrm{Rat}_{e}$ is compact if and only if $M$ has only one cusp, and that $\mathrm{Rat}_{e}$ is dense in $\mathrm{Lk}_{e} \cup \mathrm{Rat}_{e}$ (as $\Gamma \xi_{e}$ is dense in $\Lambda \Gamma$ ). Diophantine approximation in $M$ as defined in $[30 ; 31 ; 32]$ studies the rate of convergence of sequences of points in $\mathrm{Rat}_{e}$ to given points in $\mathrm{Lk}_{e}$.

For every $r$ in Rat $e_{e}$, let $D(r)$, called the depth of $r$, be the length of the subsegment of $r$ between the first and the last meeting points with $\partial V_{e}$.

Examples (1) Consider $M=\Gamma_{\mathbb{R}, \mathcal{I}} \backslash \mathbb{H}_{\mathbb{R}}^{3}$. Then $\mathcal{P}_{\Gamma_{\mathbb{R}, \mathcal{I}}} \subset \mathbb{C} \cup\{\infty\}$ is exactly $K_{-m} \cup\{\infty\}$, by Example (1) of Section 6.3. Thus, $\operatorname{Lk}_{e}(M)=\left(\Gamma_{\mathbb{R}, \mathcal{I}}\right)_{\infty} \backslash\left(\mathbb{C}-K_{-m}\right)$. In a commutative unitary ring $R$, we denote by $\left\langle p_{1}, \ldots, p_{k}\right\rangle$ the ideal generated by $p_{1}, \ldots, p_{k} \in R$. It is easy to prove as for instance in Elstrodt, Grunewald and Mennicke [18, Lemma 2.1, page 314] that $\mathrm{Rat}_{e}$ is the set of elements $r=p / q$ (modulo $\left.\left(\Gamma_{\mathbb{R}, \mathcal{I}}\right)_{\infty}\right)$ with $(p, q) \in \mathcal{O} \times \mathcal{I}$ such that $\langle p, q\rangle=\mathcal{O}$. Furthermore by [30, Lemma 2.10]

$$
D(r)=2 \log |q| \text {. }
$$


(2) Consider $M=\Gamma_{\mathbb{C}, \mathcal{I}} \backslash \mathbb{H}_{\mathbb{C}}^{n}$. Let $\mathcal{Q}(\mathbb{R})$ be the real quadric $\partial_{\infty} \mathbb{H}_{\mathbb{C}}^{n}-\{\infty\}$. By considering a basis of $K_{-m}$ over $\mathbb{Q}$, it is easy to see that $\mathcal{Q}(\mathbb{R})$ is the set of $\mathbb{R}$-points of a quadric $\mathcal{Q}$ defined over $\mathbb{Q}$ (which depends on $m$ ), whose set $\mathcal{Q}(\mathbb{Q})$ of $\mathbb{Q}$-points is $\mathcal{Q}(\mathbb{R}) \cap\left(K_{-m} \times K_{-m}^{n-1}\right)$. We have $\mathrm{Lk}_{e}=\left(\Gamma_{\mathbb{C}, \mathcal{I}}\right)_{\infty} \backslash\left(\mathcal{Q}(\mathbb{R})-\mathcal{P}_{\Gamma_{\mathbb{C}, \mathcal{I}}}\right)$. By Example (2) of Section 6.3, we have $\mathcal{P}_{\Gamma_{\mathbb{C}, \mathcal{I}}}=\mathcal{Q}(\mathbb{Q}) \cup\{\infty\}$.

Then Rat is $_{e}$ the quotient modulo $\left(\Gamma_{\mathbb{C}, \mathcal{I}}\right)_{\infty}$ of the subset of $\mathcal{Q}(\mathbb{Q})$ of points of the form $(a / c, \alpha / c)$ with $(a, \alpha, c) \in \mathcal{O} \times \mathcal{I}^{n-1} \times \mathcal{I}$ such that there exist matrices of the appropriate size $b, d, \beta, \gamma, \delta, A$ such that

$$
\left(\begin{array}{lll}
a & \gamma^{*} & b \\
\alpha & A & \beta \\
c & \delta^{*} & d
\end{array}\right)
$$

belongs to $\Gamma_{\mathbb{C}, \mathcal{I}}$. By Proposition 6.5 (2), this existence requirement is equivalent to the requirement that $q(a, \alpha, c)=0$ and $\langle a, \alpha, c\rangle=\mathcal{O}$, if $n=2$ and $\mathcal{O}=\mathcal{O}_{-m}$. By Proposition $6.5(3), \mathrm{Rat}_{e}=\left(\Gamma_{\mathbb{C}, \mathcal{I}}\right)_{\infty} \backslash \mathcal{Q}(\mathbb{Q})$ if $n=2, \mathcal{I}=\mathcal{O}=\mathcal{O}_{-m}$ and $m=$ $1,2,3,7,11,19,43,67,163$.

If $r \in \mathrm{Rat}_{e}$ is of the form $(a / c, \alpha / c)$ (modulo $\left.\left(\Gamma_{\mathbb{C}, \mathcal{I}}\right)_{\infty}\right)$ as above, then by Lemma 6.3 , we have

$$
D(r)=\log |c|+ \begin{cases}\log \operatorname{Im} \omega & \text { if } \operatorname{Re} \omega \in \mathbb{Z}, \\ \log (2 \operatorname{Im} \omega) & \text { otherwise. }\end{cases}
$$

(3) Consider $M=\Gamma_{\mathcal{I}^{\prime}} \backslash \mathbb{H}_{\mathbb{R}}^{5}$. We have $\mathcal{P}_{\Gamma_{\mathcal{I}^{\prime}}}=A(\mathbb{Q}) \cup\{\infty\}$, by Example (3) of Section 6.3. Hence $\mathrm{Lk}_{e}=\left(\Gamma_{\mathcal{I}^{\prime}}\right)_{\infty} \backslash(\mathbb{H}-A(\mathbb{Q}))$.

It is easy to see that $\mathrm{Rat}_{e}$ is the set of elements $r=p q^{-1}$ (modulo $\left(\Gamma_{\mathcal{I}^{\prime}}\right)_{\infty}$ ) with $(p, q) \in \mathcal{O}^{\prime} \times\left(\mathcal{I}^{\prime}-\{0\}\right)$ such that there exists $r, s \in \mathcal{O}^{\prime}$ with $\left|q r-q p q^{-1} s\right|=1$. Furthermore, by Lemma 6.7, we have

$$
D(r)=2 \log |q| .
$$

The cuspidal distance $d_{e}^{\prime}\left(\gamma, \gamma^{\prime}\right)$ of $\gamma, \gamma^{\prime}$ in $\operatorname{Lk}_{e} \cup \operatorname{Rat}_{e}$ is the minimum of the $\widetilde{d_{e}^{\prime}}\left(\tilde{\gamma}, \widetilde{\gamma^{\prime}}\right)$ for $\tilde{\gamma}, \widetilde{\gamma^{\prime}}$ two lifts of $\gamma, \gamma^{\prime}$ to $\widetilde{M}$ starting from $\xi_{e}$, where $\widetilde{d_{e}^{\prime}}\left(\tilde{\gamma}, \widetilde{\gamma^{\prime}}\right)$ is the greatest lower bound of $r>0$ such that the horosphere centered at $\tilde{\gamma}(+\infty)$, at signed distance $-\log 2 r$ from $\partial H_{e}$ on the geodesic line $] \xi_{e}, \widetilde{\gamma}(+\infty)$ [, meets $\widetilde{\gamma^{\prime}}$ [30, Section 2.1]. Although $\widetilde{d_{e}^{\prime}}$ is not necessarily an actual distance, it is equivalent to the Hamenstädt distance (see Section 3.1 and Hersonsky and Paulin [30, Remark 2.6]).

Examples (1) If $M$ has constant curvature -1, if one identifies $\mathrm{Lk}_{e} \cup \mathrm{Rat}_{e}$ with a subset of $\partial H_{e}$ by the first intersection point, then $d_{e}^{\prime}$ is the induced Riemannian distance on $\partial H_{e}$, which is Euclidean [30, Section 2.1]; in particular, if $M=\Gamma_{\mathbb{R}, \mathcal{I}} \backslash \mathbb{H}_{\mathbb{R}}^{3}$ or 
$M=\Gamma_{\mathcal{I}^{\prime}} \backslash \mathbb{H}_{\mathbb{R}}^{5}$, then $d_{e}^{\prime}$ is the quotient of the standard Euclidean distance on $\mathrm{Lk}_{e} \cup$ Rat $_{e}$ identified with a subset of $\left(\Gamma_{\mathbb{R}, \mathcal{I}}\right)_{\infty} \backslash \mathbb{C}$ or $\left(\Gamma_{\mathcal{I}^{\prime}}\right)_{\infty} \backslash \mathbb{H}$.

(2) If $M$ is Hermitian with constant holomorphic sectional curvature -1 , then $d_{e}^{\prime}$ is no longer Riemannian, but by Proposition 6.2, it is a multiple of the modified Cygan distance $d_{\text {Cyg }}^{\prime}$. In particular, if $M=\Gamma_{\mathbb{C}, \mathcal{I}} \backslash \mathbb{H}_{\mathbb{C}}^{n}$, then $d_{e}^{\prime}$ is the quotient by $\left(\Gamma_{\mathbb{C}, \mathcal{I}}\right)_{\infty}$ acting on $\partial_{\infty} \mathbb{H}_{\mathbb{C}}^{n}$ of the distances

$$
\left\{\begin{array}{l}
\frac{1}{2 \sqrt{\operatorname{Im} \omega}} d_{\text {Cyg }}^{\prime} \quad \text { if } \operatorname{Re} \omega \in \mathbb{Z}, \\
\frac{1}{2 \sqrt{2 \operatorname{Im} \omega}} d_{\text {Cyg }}^{\prime} \text { otherwise } .
\end{array}\right.
$$

Remark The claim in the first paragraph of Section 3.11 in [31] (where the authors only considered the case $m=1$ and $\mathcal{I}=\mathcal{O}=\mathcal{O}_{-1}$ ) that the cuspidal distance coincides with the Hamenstädt distance is incorrect. But every statement remains correct. Since the Cygan distance and the modified Cygan distance are equivalent, this does not change the statement of the main results, Theorems 3.1, 3.2, 3.4, and 3.5 of [31]. Since $d_{\text {Cyg }}^{\prime} \leq \sqrt{2} d_{\text {Cyg }}$ and $d_{e}^{\prime}=\frac{1}{2} d_{\text {Cyg }}^{\prime}$ by the above displayed formula with $\omega=i$, so that $d_{\text {Cyg }} \geq \sqrt{2} d_{e}^{\prime}$, Theorem 3.6 of [31] also remains correct, using in its proof the inequality $d_{\text {Cyg }} \geq \sqrt{2} d_{e}^{\prime}$ instead of the equality $d_{\text {Cyg }}=\sqrt{2} d_{\infty}$ mentioned there.

With $M$ as in the beginning of this subsection, for every $x$ in $\mathrm{Lk}_{e}$, define the approximation constant $c(x)$ of $x$ as

$$
c(x)=\liminf _{r \in \operatorname{Rat}_{e}, D(r) \rightarrow \infty} d_{e}^{\prime}(x, r) e^{D(r)} .
$$

The Lagrange spectrum of $M$ with respect to $e$ is the subset $\operatorname{Sp}_{\mathrm{Lag}}(M, e)$ of $\mathbb{R}$ consisting of the constants $c(x)$ for $x$ in $\mathrm{Lk}_{e}$. It is shown in [30] that

- $c(x)$ is well defined for any $x$ in $\mathrm{Lk}_{e}$ (as Rat $\mathrm{R}_{e}$ is dense in $\mathrm{Lk}_{e} \cup \mathrm{Rat}_{e}$ and $\left\{D(r): r \in \mathrm{Rat}_{e}\right\}$ is a discrete subset of $\mathbb{R}$ with finite multiplicities),

- $c(x)$ is finite for any $x$ in $\mathrm{Lk}_{e}$ (as $x$ is nonwandering) (note that if $y$ is a locally geodesic line starting from $e$ in $M$ that converges into a cusp of $M$, then the same formula would yield $c(y)=+\infty)$,

- the least upper bound of $\operatorname{Sp}_{\mathrm{Lag}}(M, e)$, denoted by $K_{M, e}$ and called the Hurwitz constant of $(M, e)$, is finite.

In particular, $\operatorname{Sp}_{\mathrm{Lag}}(M, e) \subset\left[0, K_{M, e}\right]$. The following result tells us that the Lagrange spectrum contains a nontrivial initial interval $[0, c]$, with a universal lower bound on $c$ (whose optimal value we do not know). 
Theorem 6.10 Let $M$ be a complete, nonelementary, geometrically finite Riemannian manifold with sectional curvature at most -1 and dimension at least 3 , and let $e$ be a cusp of $M$ endowed with a Margulis neighbourhood. The Lagrange spectrum $\operatorname{Sp}_{\text {Lag }}(M, e)$ contains the interval [0, 0.00057]. In particular, $K_{M, e} \geq 0.00057$.

Proof By [30], the map $h \mapsto \frac{1}{2} e^{-h}$ maps the asymptotic height spectrum bijectively onto the Lagrange spectrum. We apply Corollary 5.15 and the computation above it.

A precise version of this theorem is stated as corollaire 5 in our paper [48] when $M$ is a real or complex hyperbolic manifold. Note that the constant $c_{*}$ of [48], which satisfies $c_{*}=e^{-h_{*}} / 2$ with $h_{*} \approx 6.7771$, is approximatively 0.00057 , and not 0.0337 as indicated by mistake in [48]. Theorem 1.7 in the introduction follows immediately, by the first example discussed in this section. By varying the (nonuniform) arithmetic lattices in the isometry group of a negatively curved symmetric space (see for instance Maclachlan and Reid [42] or Maclachlan, Waterman and Wielenberg [43]), other arithmetic applications are possible. We only state two of them in what follows; see also our paper [48].

Let $\mathcal{I}^{\prime}$ be a nonzero two-sided ideal in an order $\mathcal{O}^{\prime}$ of a quaternion algebra $A(\mathbb{Q})$ over $\mathbb{Q}$ ramifying over $\mathbb{R}$, and let $N$ be the reduced norm on $A(\mathbb{R})=A(\mathbb{Q}) \otimes_{\mathbb{Q}} \mathbb{R}$ (see for instance Vignéras [61] and Section 6.2). For every $x \in A(\mathbb{R})-A(\mathbb{Q})$, define the approximation constant of $x$ by

$$
\begin{aligned}
& c(x)=\quad \liminf _{(p, q) \in \mathcal{O}^{\prime} \times \mathcal{I}^{\prime}:} \quad N(q) N\left(x-p q^{-1}\right)^{\frac{1}{2}}, \\
& \exists r, s \in \mathcal{O}^{\prime} N\left(q r-q p q^{-1} s\right)=1, N(q) \rightarrow \infty
\end{aligned}
$$

and the Hamilton-Lagrange spectrum for the approximation of elements of $\mathbb{H}$ by elements of $\mathcal{O}^{\prime} \mathcal{I}^{\prime-1}$ as the subset of $\mathbb{R}$ consisting of the $c(x)$ for $x \in A(\mathbb{R})-A(\mathbb{Q})$. Note that $c(x)$ is finite if $x \notin A(\mathbb{Q})$, as then $x$ is not a parabolic fixed point of $\Gamma_{\mathcal{I}^{\prime}}$. Apply Theorem 6.10 to $M=\Gamma_{\mathcal{I}^{\prime}} \backslash \mathbb{H}_{\mathbb{R}}^{5}$ with the above discussions of the third example to get the following result.

\section{Theorem 6.11 The Hamilton-Lagrange spectra contain the interval [0, 0.00057].}

In the case when $\mathcal{I}^{\prime}=\mathcal{O}^{\prime}$ and $\mathcal{O}^{\prime}$ is the Hurwitz maximal order in Hamilton's quaternion algebra $A(\mathbb{Q}) \subset \mathbb{H}$ (see Section 6.2), A Schmidt [54] proved that the HamiltonLagrange spectrum contains $\sqrt{2} \mathrm{Sp}_{\mathbb{Q}}$ where $\mathrm{Sp}_{\mathbb{Q}}$ is the classical Lagrange spectrum for the approximation of real numbers by rational numbers. As $\operatorname{Sp}_{\mathbb{Q}}$ contains $[0, \mu]$ where $\mu$ is Freiman's constant (see the end of Section 5.4), this proves that the HamiltonLagrange spectrum in this case contains the interval [0,0.312]. Note that the fact that our approximation constant coincides with the inverse of A Schmidt's approximation constant follows from Schmidt [53, Theorem 5]. 
Let $m$ be a squarefree positive integer, let $\mathcal{I}$ be a nonzero ideal in an order $\mathcal{O}$ in the ring of integers $\mathcal{O}_{-m}$ of the imaginary quadratic number field $\mathbb{Q}(i \sqrt{m})$, and let $\omega$ be an element of $\mathcal{O}_{-m}$ with $\operatorname{Im} \omega>0$ such that $\mathcal{O}=\mathbb{Z}+\omega \mathbb{Z}$. Let $\mathcal{E}_{\mathcal{O}, \mathcal{I}}$ be the set of $(a, \alpha, c)$ in $\mathcal{O} \times \mathcal{I}^{n-1} \times \mathcal{I}$ such that there exists a matrix of the form

$$
\left(\begin{array}{lll}
a & \gamma^{*} & b \\
\alpha & A & \beta \\
c & \delta^{*} & d
\end{array}\right)
$$

that belongs to $\Gamma_{\mathbb{C}, \mathcal{I}}$. If $n=2$ and $\mathcal{O}=\mathcal{O}_{-m}$, then, as seen previously,

$$
\mathcal{E}_{\mathcal{O}, \mathcal{I}}=\left\{(a, \alpha, c) \in \mathcal{O} \times \mathcal{I}^{n-1} \times \mathcal{I}: q(a, \alpha, c)=0,\langle a, \alpha, c\rangle=\mathcal{O}\right\} .
$$

We do not know if this is the case for every $n, \mathcal{I}, \mathcal{O}$ as above. For every $x \in \mathcal{Q}(\mathbb{R})-$ $\mathcal{Q}(\mathbb{Q})$, define the approximation constant of $x$ by

$$
c(x)=\liminf _{(a, \alpha, c) \in \mathcal{E}_{\mathcal{O}, \mathcal{I}},|c| \rightarrow \infty}|c| d_{\text {Cyg }}^{\prime}(x,(a / c, \alpha / c)),
$$

and the Heisenberg-Lagrange spectrum, for the approximation of elements of $\mathcal{Q}(\mathbb{R})$ by elements of $\left\{(a / c, \alpha / c):(a, \alpha, c) \in \mathcal{E}_{\mathcal{O}, \mathcal{I}}\right\} \subset \mathcal{Q}(\mathbb{Q})$, as the subset of $\mathbb{R}$ consisting of the $c(x)$ for $x \in \mathcal{Q}(\mathbb{R})-\mathcal{Q}(\mathbb{Q})$. Note that $c(x)$ is finite if $x \notin \mathcal{Q}(\mathbb{Q})$, as then $x$ is not a parabolic fixed point of $\Gamma_{\mathbb{C}, \mathcal{I}}$. Our last result follows from Theorem 6.10 and the previous discussions of the second example.

Theorem 6.12 If $\operatorname{Re} \omega \in \mathbb{Z}$, the Heisenberg-Lagrange spectra contain the interval $[0,0.0011 / \sqrt{\operatorname{Im} \omega}]$ and otherwise contain $[0,0.0008 / \sqrt{\operatorname{Im} \omega}]$.

Theorem 1.8 in the introduction follows from this one (and from Lemma 6.1) by taking $m=1, n=2, \mathcal{I}=\mathcal{O}=\mathcal{O}_{-1}$, as then $\operatorname{Im} \omega=1$.

\section{References}

[1] W Abikoff, B Maskit, Geometric decompositions of Kleinian groups, Amer. J. Math. 99 (1977) 687-697 MR0480992

[2] L V Ahlfors, Möbius transformations and Clifford numbers, from: "Differential geometry and complex analysis", (I Chavel, H M Farkas, editors), Springer, Berlin (1985) 65-73 MR780036

[3] C S Aravinda, E Leuzinger, Bounded geodesics in rank-1 locally symmetric spaces, Ergodic Theory Dynam. Systems 15 (1995) 813-820 MR1356615

[4] H Aslaksen, Quaternionic determinants, Math. Intelligencer 18 (1996) 57-65 MR1412993 
[5] A F Beardon, The geometry of discrete groups, Graduate Texts in Math. 91, Springer, New York (1983) MR698777

[6] A Borel, Linear algebraic groups, from: "Algebraic Groups and Discontinuous Subgroups (Proc. Sympos. Pure Math., Boulder, Colo., 1965)”, Amer. Math. Soc. (1966) 3-19 MR0204532

[7] A Borel, Reduction theory for arithmetic groups, from: "Algebraic Groups and Discontinuous Subgroups (Proc. Sympos. Pure Math., Boulder, Colo., 1965)”, Amer. Math. Soc. (1966) 20-25 MR0204533

[8] A Borel, Harish-Chandra, Arithmetic subgroups of algebraic groups, Ann. of Math. (2) 75 (1962) 485-535 MR0147566

[9] M Bourdon, Sur le birapport au bord des CAT(-1)-espaces, Inst. Hautes Études Sci. Publ. Math. 83 (1996) 95-104 MR1423021

[10] B H Bowditch, Geometrical finiteness with variable negative curvature, Duke Math. J. 77 (1995) 229-274 MR1317633

[11] M R Bridson, A Haefliger, Metric spaces of non-positive curvature, Grund. der Math. Wissenschaften 319, Springer, Berlin (1999) MR1744486

[12] P Buser, H Karcher, Gromov's almost flat manifolds, Astérisque 81, Société Mathématique de France, Paris (1981) MR619537

[13] S Buyalo, V Schroeder, M Walz, Geodesics avoiding open subsets in surfaces of negative curvature, Ergodic Theory Dynam. Systems 20 (2000) 991-1006 MR1779390

[14] D A Cox, Primes of the form $x^{2}+n y^{2}$, Fermat, class field theory and complex multiplication, Wiley-Interscience, John Wiley \& Sons, New York (1989) MR1028322

[15] T W Cusick, M E Flahive, The Markoff and Lagrange spectra, Math. Surveys and Monogr. 30, Amer. Math. Soc. (1989) MR1010419

[16] S G Dani, Bounded orbits of flows on homogeneous spaces, Comment. Math. Helv. 61 (1986) 636-660 MR870710

[17] J Dieudonné, Les déterminants sur un corps non commutatif, Bull. Soc. Math. France 71 (1943) 27-45 MR0012273

[18] J Elstrodt, F Grunewald, J Mennicke, Groups acting on hyperbolic space. Harmonic analysis and number theory, Springer Monogr. in Math., Springer, Berlin (1998) MR1483315

[19] J-M Feustel, Über die Spitzen von Modulflächen zur zweidimensionalen komplexen Einheitskugel, Preprint Series 13 Akad. Wiss. DDR, ZIMM, Berlin 14 (1977)

[20] L R Ford, Rational approximations to irrational complex numbers, Trans. Amer. Math. Soc. 19 (1918) 1-42 MR1501085

[21] G A Freiman, Diophantine approximations and the geometry of numbers (Markov's problem), Kalinin. Gosudarstv. Univ., Kalinin (1975) MR0485714 
[22] É Ghys, P de la Harpe, editors, Sur les groupes hyperboliques d'après Mikhael Gromov, Progress in Math. 83, Birkhäuser, Boston (1990) MR1086648

[23] W M Goldman, Complex hyperbolic geometry, Oxford Math. Monogr., Oxford Univ. Press (1999) MR1695450

[24] M Hall, Jr, On the sum and product of continued fractions, Ann. of Math. (2) 48 (1947) 966-993 MR0022568

[25] M Hall, Jr, The Markoff spectrum, Acta Arith. 18 (1971) 387-399 MR0296023

[26] U Hamenstädt, A new description of the Bowen-Margulis measure, Ergodic Theory Dynam. Systems 9 (1989) 455-464 MR1016663

[27] Y Hellegouarch, Quaternionic homographies: application to Ford hyperspheres, C. R. Math. Rep. Acad. Sci. Canada 11 (1989) 171-176 MR1010923

[28] S Hersonsky, F Paulin, On the volumes of complex hyperbolic manifolds, Duke Math. J. 84 (1996) 719-737 MR1408542

[29] S Hersonsky, F Paulin, On the rigidity of discrete isometry groups of negatively curved spaces, Comment. Math. Helv. 72 (1997) 349-388 MR1476054

[30] S Hersonsky, F Paulin, Diophantine approximation for negatively curved manifolds, Math. Z. 241 (2002) 181-226 MR1930990

[31] S Hersonsky, F Paulin, Diophantine approximation in negatively curved manifolds and in the Heisenberg group, from: "Rigidity in dynamics and geometry (Cambridge, 2000)", (M Burger, A Iozzi, editors), Springer, Berlin (2002) 203-226 MR1919402

[32] S Hersonsky, F Paulin, Counting orbit points in coverings of negatively curved manifolds and Hausdorff dimension of cusp excursions, Ergodic Theory Dynam. Systems 24 (2004) 803-824 MR2060999

[33] S Hersonsky, F Paulin, On the almost sure spiraling of geodesics in negatively curved manifolds, to appear in J. Diff. Geom. arXiv:0708.3389

[34] R Hill, S L Velani, The Jarník-Besicovitch theorem for geometrically finite Kleinian groups, Proc. London Math. Soc. (3) 77 (1998) 524-550 MR1643409

[35] R-P Holzapfel, Arithmetische Kugelquotientenflächen I/II, Seminarberichte 14, Humboldt Univ. Sekt. Math., Berlin (1978) MR521083

[36] R-P Holzapfel, Ball and surface arithmetics, Aspects of Math., E29, Friedr. Vieweg \& Sohn, Braunschweig (1998) MR1685419

[37] S Kamiya, On discrete subgroups of $\mathrm{PU}(1,2 ; \mathbf{C})$ with Heisenberg translations, J. London Math. Soc. (2) 62 (2000) 827-842 MR1794288

[38] R Kellerhals, Quaternions and some global properties of hyperbolic 5-manifolds, Canad. J. Math. 55 (2003) 1080-1099 MR2005283

[39] D Y Kleinbock, G A Margulis, Logarithm laws for flows on homogeneous spaces, Invent. Math. 138 (1999) 451-494 MR1719827 
[40] D Y Kleinbock, B Weiss, Bounded geodesics in moduli space, Int. Math. Res. Not. 30 (2004) 1551-1560 MR2049831

[41] A Korányi, H M Reimann, Quasiconformal mappings on the Heisenberg group, Invent. Math. 80 (1985) 309-338 MR788413

[42] C Maclachlan, A W Reid, The arithmetic of hyperbolic 3-manifolds, Graduate Texts in Math. 219, Springer, New York (2003) MR1937957

[43] C Maclachlan, P L Waterman, N Wielenberg, Higher-dimensional analogues of the modular and Picard groups, Trans. Amer. Math. Soc. 312 (1989) 739-753 MR965301

[44] K Matsuzaki, M Taniguchi, Hyperbolic manifolds and Kleinian groups, Oxford Math. Monogr., Oxford Univ. Press (1998) MR1638795

[45] J-P Otal, Sur la géometrie symplectique de l'espace des géodésiques d'une variété à courbure négative, Rev. Mat. Iberoamericana 8 (1992) 441-456 MR1202417

[46] J R Parker, Shimizu's lemma for complex hyperbolic space, Internat. J. Math. 3 (1992) 291-308 MR1146815

[47] J Parkkonen, F Paulin, Unclouding the sky of negatively curved manifolds, Geom. Funct. Anal. 15 (2005) 491-533 MR2153908

[48] J Parkkonen, F Paulin, Sur les rayons de Hall en approximation diophantienne, C. R. Math. Acad. Sci. Paris 344 (2007) 611-614 MR2334070

[49] J Parkkonen, F Paulin, Spiraling spectra of geodesic lines in negatively curved manifolds, Preprint, Univ. Jyväskylä (2008) arXiv:0806.1857

[50] J Parkkonen, F Paulin, On strictly convex subsets in negatively curved manifolds, in preparation

[51] F Paulin, Un groupe hyperbolique est déterminé par son bord, J. London Math. Soc. (2) 54 (1996) 50-74 MR1395067

[52] G Poitou, Sur l'approximation des nombres complexes par les nombres des corps imaginaires quadratiques dénués d'idéaux non principaux, particulièrement lorsque vaut l'algorithme d'Euclide, Ann. Sci. Ecole Norm. Sup. (3) 70 (1953) 199-265 MR0066431

[53] A L Schmidt, Farey simplices in the space of quaternions, Math. Scand. 24 (1969) 31-65 MR0253993

[54] A L Schmidt, On the approximation of quaternions, Math. Scand. 34 (1974) 184-186 MR0371823

[55] T A Schmidt, M Sheingorn, Riemann surfaces have Hall rays at each cusp, Illinois J. Math. 41 (1997) 378-397 MR1458179

[56] V Schroeder, Bounded geodesics in manifolds of negative curvature, Math. Z. 235 (2000) 817-828 MR1801585 
[57] C Series, The modular surface and continued fractions, J. London Math. Soc. (2) 31 (1985) 69-80 MR810563

[58] N J A Sloane, editor, The online encyclopedia of integer sequences Available at http://www.research.att.com/ njas/sequences/A118472

[59] B Stratmann, The Hausdorff dimension of bounded geodesics on geometrically finite manifolds, Ergodic Theory Dynam. Systems 17 (1997) 227-246 MR1440778

[60] D Sullivan, Disjoint spheres, approximation by imaginary quadratic numbers, and the logarithm law for geodesics, Acta Math. 149 (1982) 215-237 MR688349

[61] M-F Vignéras, Arithmétique des algèbres de quaternions, Lecture Notes in Math. 800, Springer, Berlin (1980) MR580949

[62] R Walter, Some analytical properties of geodesically convex sets, Abh. Math. Sem. Univ. Hamburg 45 (1976) 263-282 MR0417984

[63] T Zink, Über die Anzahl der Spitzen einiger arithmetischer Untergruppen unitärer Gruppen, Math. Nachr. 89 (1979) 315-320 MR546890

Department of Mathematics and Statistics, PO Box 35

40014 University of Jyväskylä, Finland

Département de Mathématique et Applications, UMR 8553 CNRS, Ecole Normale Supérieure 45 rue d'Ulm, 75230 PARIS Cedex 05, France

parkkone@maths.jyu.fi, Frederic.Paulin@ens.fr

Proposed: Martin Bridson

Received: 1 June 2007

Seconded: Walter Neumann, Jean-Pierre Otal

Revised: 21 July 2009 